\title{
Impact of Intelligent Transportation Systems on Parallel Hybrid Electric Heavy Duty Vehicles
}

\author{
Ahmed Shihab Ahmed Al-Samari
}

Follow this and additional works at: https://researchrepository.wvu.edu/etd

\section{Recommended Citation}

Al-Samari, Ahmed Shihab Ahmed, "Impact of Intelligent Transportation Systems on Parallel Hybrid Electric Heavy Duty Vehicles" (2014). Graduate Theses, Dissertations, and Problem Reports. 5087.

https://researchrepository.wvu.edu/etd/5087

This Dissertation is protected by copyright and/or related rights. It has been brought to you by the The Research Repository @ WVU with permission from the rights-holder(s). You are free to use this Dissertation in any way that is permitted by the copyright and related rights legislation that applies to your use. For other uses you must obtain permission from the rights-holder(s) directly, unless additional rights are indicated by a Creative Commons license in the record and/ or on the work itself. This Dissertation has been accepted for inclusion in WVU Graduate Theses, Dissertations, and Problem Reports collection by an authorized administrator of The Research Repository @ WVU.

For more information, please contact researchrepository@mail.wvu.edu. 


\title{
Impact of Intelligent Transportation Systems on Parallel Hybrid Electric Heavy Duty Vehicles
}

\author{
Ahmed Shihab Ahmed Al-Samari \\ Dissertation submitted to the \\ Benjamin M. Statler College of Engineering and Mineral Resources \\ West Virginia University \\ In partial fulfillment of the requirements for the degree of \\ Doctor of Philosophy In \\ Mechanical Engineering \\ Nigel Clark, Ph.D., Chair \\ Gregory J. Thompson, Ph.D. \\ Hailin Li, Ph.D. \\ W. Scott Wayne, Ph.D. \\ Wathiq Abdul-Razzaq, Ph.D. \\ West Virginia University \\ Mechanical and Aerospace Engineering Department \\ Morgantown, WV 26505-6106
}

2014

Keywords: Looking-ahead Control Strategy, Intelligent Transportation Systems, Hybrid Electric Truck, Parallel Hybrid Electric Vehicle, Fuel Economy, Powertrain Management for HEVs. Copyright 2014, Ahmed Al-Samari 


\title{
Abstract \\ Impact of Intelligent Transportation Systems on Parallel Hybrid Electric Heavy Duty Vehicles
}

\begin{abstract}
Ahmed Al-Samari
A hybrid electric vehicle uses multiple sources of energy that can be independently or all together used to propel the wheels. In the presented work, the vehicle propulsion controller (VPC) for a parallel heavy duty hybrid electric vehicle (HEV) model has been modified to manage the alternative power source in advance based on the forthcoming traffic information. The goal is to prepare the powertrain for the next power event by making more energy storage capacity to capture free energy via regenerative braking or store more energy for expected need. The method of preparation will be by managing the battery state of charge (SOC), which is a metal hydride battery for this study, to take advantage of opportunistic regeneration. Autonomie software was used to simulate parallel HEV models.
\end{abstract}

The results revealed that the proposed looking-ahead control strategy for a class 8 parallel hybrid heavy duty vehicle with an engine power of $410 \mathrm{~kW}$ had a substantial contribution in preparing the system for forthcoming power demand. The looking-ahead strategy employed in this study improved fuel economy from $0.5 \%$ on flat terrain to about $3 \%$ on mountain terrain. Moreover, a looking-ahead strategy can contribute significantly to maintaining adequate power for the vehicle on different terrain types. The engine power can be downsized (with looking-ahead strategy) therefore improving fuel economy up to $13 \%$ while maintaining adequate power over different terrain types. The battery energy capacity can be downsized (with looking-ahead strategy) by half while maintaining nearly the same benefits (i.e. fuel economy and adequate power) compared to the hybridization system without looking-ahead strategy. Since different routes types (i.e. flat, hilly and mountain terrains) were used to investigate the impact of the lookingahead strategy on heavy duty parallel HEV, these results can generally be applied to many terrain and traffic situations. 


\section{Acknowledgements}

This research was supported by the Higher Committee of Education Development (HCED) in Iraq. I appreciated my sponsor's support to finish my study successfully and earn this degree, and I ask Allah to guide and enable me to serve my country Iraq. I am extremely grateful to my advisor Dr. Nigel Clark for his guidance, and encouragement throughout the research. I really appreciate his broad knowledge and his capability to find out an exit from every difficulty I was facing through my research. I would like to express my sincere appreciation to my other committee members Dr. Scott Wayne, Dr. Gregory J. Thompson, Dr. Hailin Li and Dr. Wathiq Abdul-Razzaq for their invaluable comments and suggestions. I have benefitted greatly from their advice.

I would like to thank my parents for their support, and I am asking Allah to bless them and grant them the paradise. I would like to thank my sincere wife, kids, brothers, and sisters for supporting me emotionally and giving me a reason to continue my tough academic learning journey. I would like to thank all of my relatives and my friends for asking and encouraging me on finishing my study.

I dedicate my success to my uncle the martyr (assassinated) Abdul-Rahman Al-Samari. He was always contacting me and telling me that "Ahmed we want to be proud of your degree and may Allah ease your study". 


\section{Table of Contents}

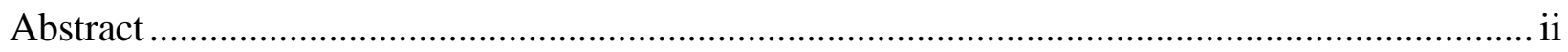

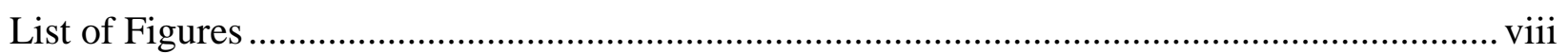

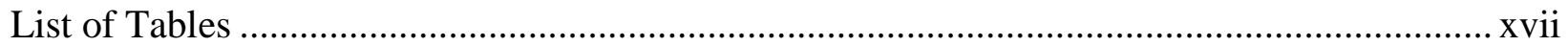

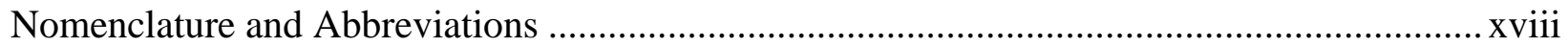

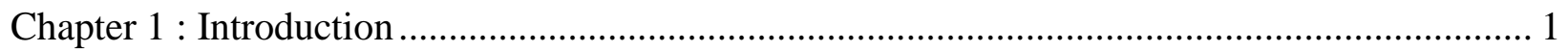

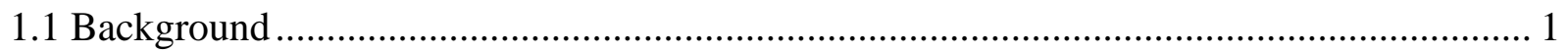

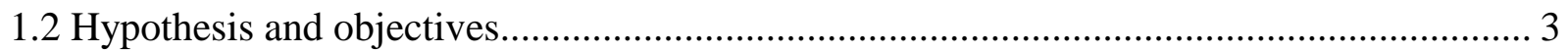

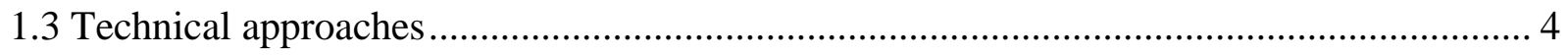

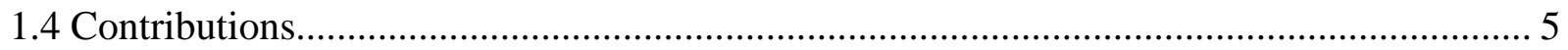

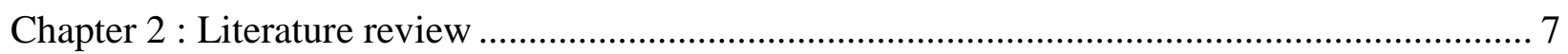

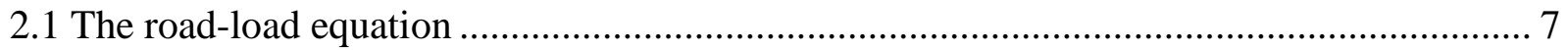

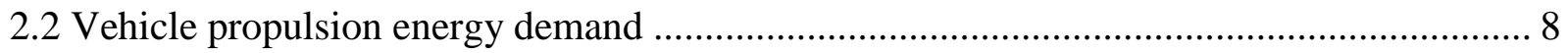

2.3 Hybrid electric vehicle …………………………..................................................... 8

2.4 Control systems for improving drivability .................................................................... 9

2.4.1 Empirical rule-based control strategy ……………………........................................ 10

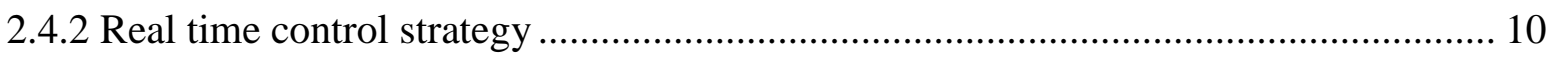

2.5 Look-ahead control for hybrid electric vehicle ................................................................. 11

2.6 Backward-looking models (SIMPLEV, ADVISOR) .................................................... 12

2.7 Forward-looking model (Autonomie) ............................................................................ 12

2.8 Energy storage system for hybrid electric vehicles ......................................................... 13

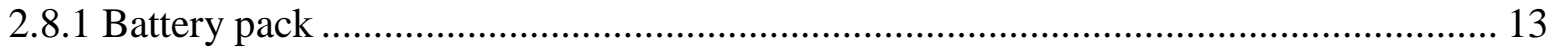

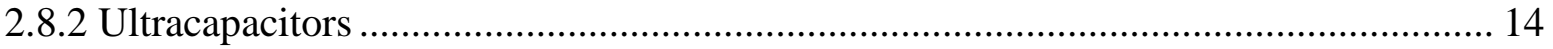

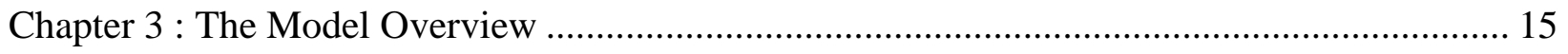

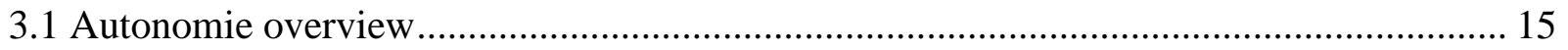


3.2 Powertrain controller strategy for a direct parallel pre-transmission HEV .................... 16

3.2.1 The vehicle propulsion controller design description ........................................ 19

3.2.2 Parallel HEV power sources management..................................................... 20

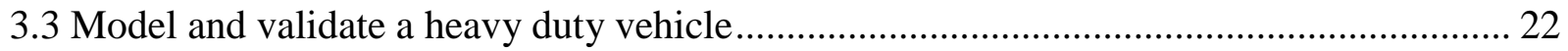

3.3.1 The tested conventional truck information .................................................. 23

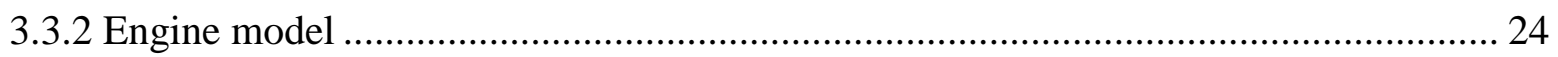

3.3.3 Validate simulation results...................................................................... 25

3.4 Several uphill and downhill terrains with highway constant speed cycles ...................... 29

3.5 Identify different real world driving cycles for heavy duty vehicles............................ 31

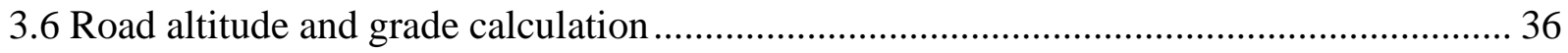

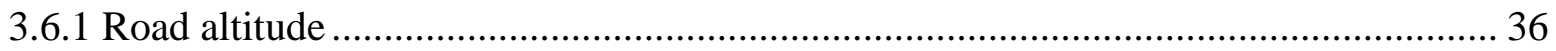

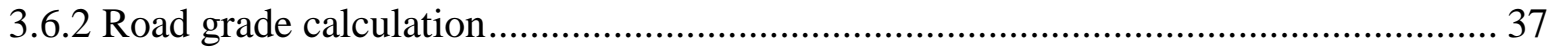

3.7 Generating road grade patterns and matching them with the chassis driving cycles such as

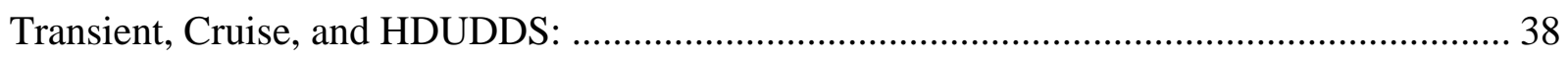

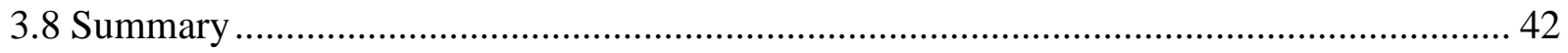

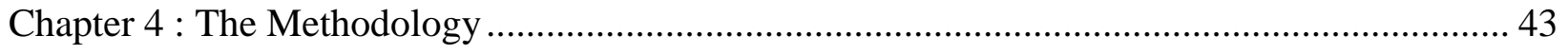

4.1 Modifying the VPC for a direct parallel HEV model............................................... 43

4.1.1 Conventional vehicle propulsion controller ................................................... 43

4.1.2 Modified vehicle propulsion controller .......................................................... 44

4.2 Algorithm of the modification on vehicle propulsion controller ................................. 45

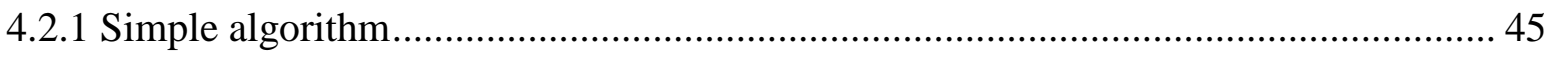

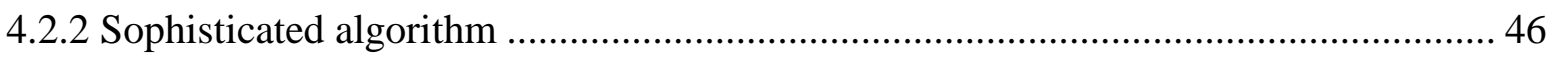

4.3 Scenarios to study the impact of ITS information ahead.......................................... 47

4.4 Hybridization equipment efficiency .................................................................. 48

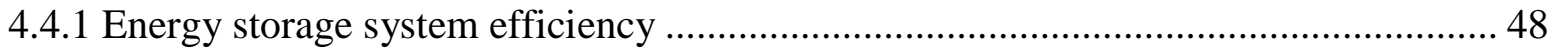


4.4.2 Electric motor efficiency.

4.5 Calculation of time needed for charging and discharging electric storage system............ 51

4.6 Estimate the negative road grade when driver needs to depress the brake....................... 52

4.6.1 Energy expected using the brake system for truck in comparison to light duty .......... 54

4.6.2 Impact of aerodynamic and tire improvement on increasing use of the brake ........... 56

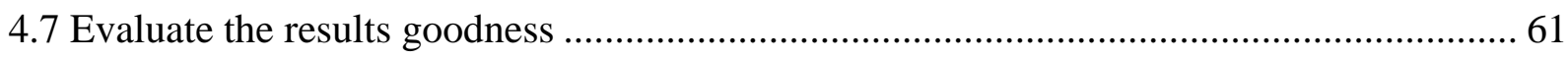

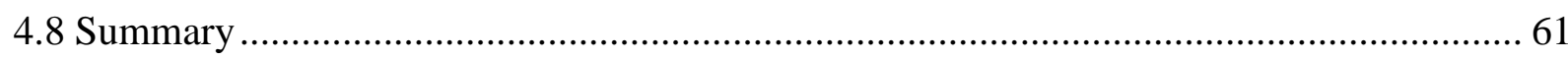

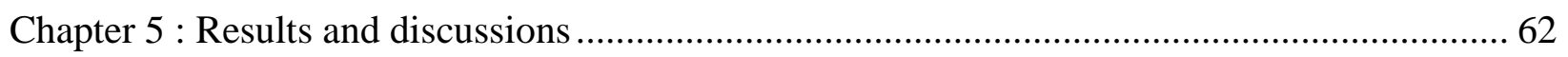

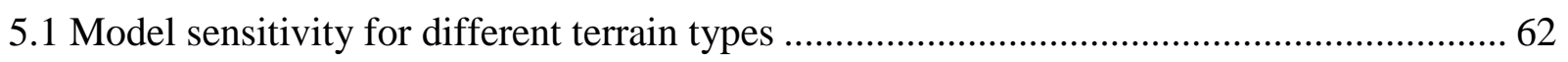

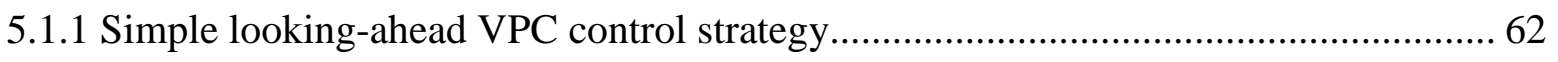

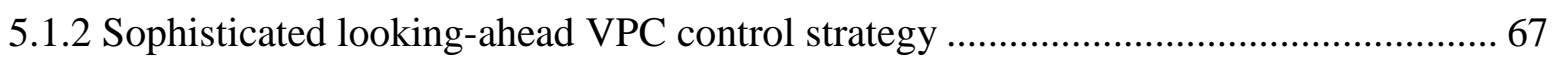

5.2 The impact of looking-ahead control strategy for parallel HEV on fuel economy and

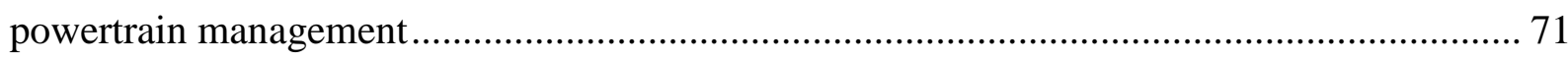

5.2.1 Ascending and descending hill constant speed driving cycles ............................. 71

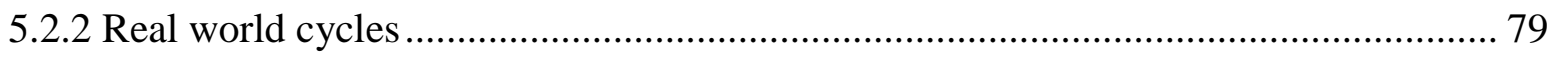

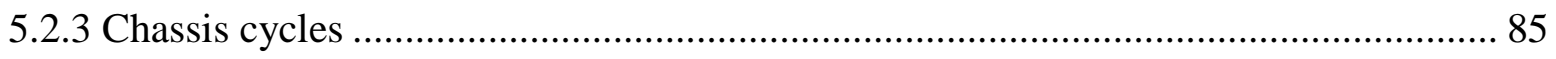

5.3 The contribution of looking-ahead VPC on energy storage system effectiveness ........... 89

5.4 The ability of suggested control strategy to improve maintaining adequate power during

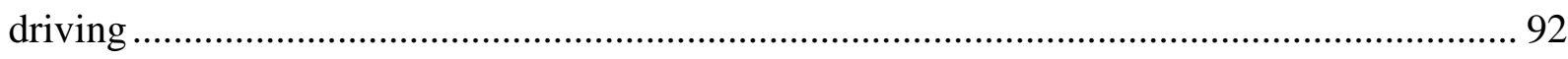

5.5 Looking-ahead strategy impact on maintaining adequate power for different real world

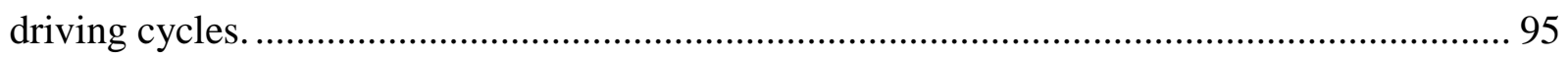

5.6 Downsizing engine power opportunity with looking-ahead strategy ............................. 98

5.7 Simulation results goodness for different driving cycles......................................... 112

5.8 Downsizing ESS energy opportunity with looking-ahead strategy ............................. 115

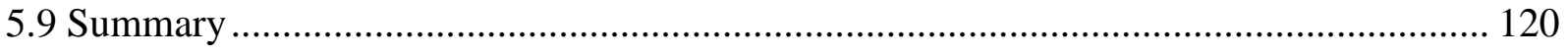

Chapter 6 : Conclusions and Recommendations ...................................................... 121 


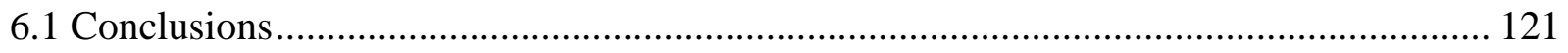

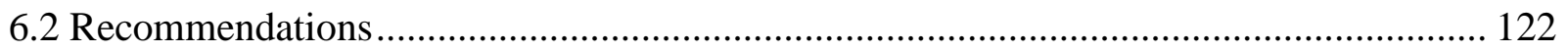

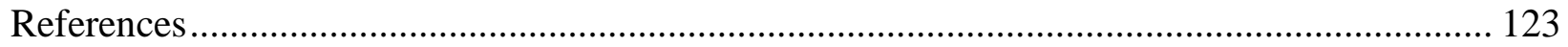

Appendix A: Simulation results goodness ................................................................... 129

A.1: Conventional vehicle at full load ................................................................... 129

A.2: HEV_150_10_with NoITS at full load ................................................................ 130

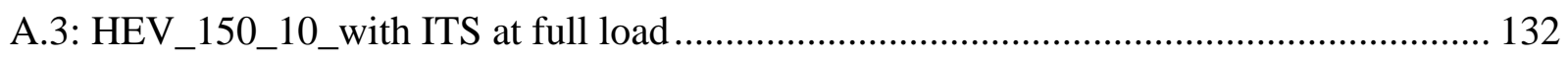

Appendix B: Evaluate the worth of parallel heavy duty HEV relative to conventional heavy duty

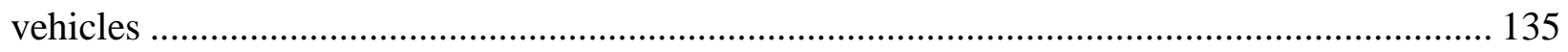

Appendix C: Energy losses and equipment efficiencies .................................................... 138

Appendix D: Matlab program code built by the researcher................................................. 148 


\section{List of Figures}

Figure 3.1: Schematic of conventional truck model in Autonomie [54] ..................................... 15

Figure 3.2: Schematic of pre-transmission hybrid drives [23] .................................................. 16

Figure 3.3: The control strategy for direct parallel pre-transmission HEV [54] ......................... 17

Figure 3.4: Diagram for subsystem control strategy for direct parallel pre-transmission HEV [54]

Figure 3.5: State flow (chart) for the VPC of parallel pre-transmission hybrid electric vehicle

Figure 3.6: State flow (sub chart) for the VPC of parallel pre-transmission hybrid electric vehicle to control engine on/off status [54]. 21

Figure 3.7: State flow (sub chart) for the VPC of parallel pre-transmission hybrid electric vehicle to manage the powertrain at stop or the brake status [54] ... 21

Figure 3.8: State flow (sub chart) for the VPC of parallel pre-transmission hybrid electric vehicle

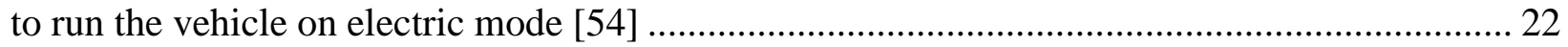

Figure 3.9: General Simulink diagram of the engine model in Autonomie [54] ......................... 25

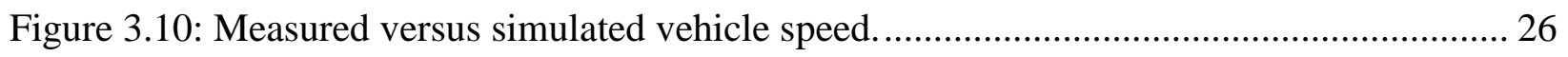

Figure 3.11: Measured versus simulated engine torque........................................................... 27

Figure 3.12: Measured versus simulated engine speed............................................................. 27

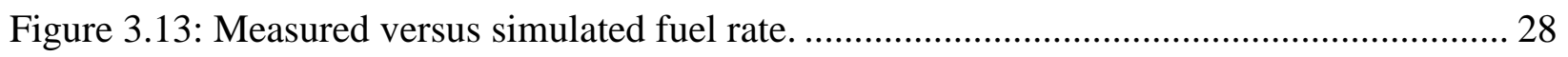

Figure 3.14: Measured versus simulated output engine power.................................................. 28

Figure 3.15: Ascending hill with 3\% road grade ...................................................................... 29

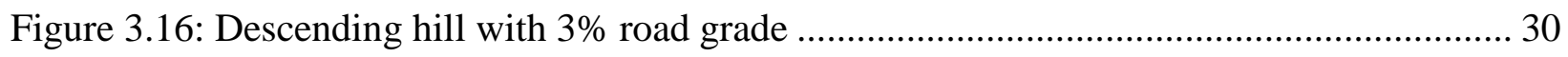

Figure 3.17: Descending and Ascending hill with 3\% road grade ............................................. 30

Figure 3.18: Ascending and Descending hill with $0.5 \%, 2 \%$ and 3\% road grades respectively .. 31

Figure 3.19: Columbus, $\mathrm{OH}$ to Indianapolis, IN real world driving cycle [56]........................... 33

Figure 3.20: Las Vegas, NV to Halloran Summit Road, Nipton, CA real world cycle [56] ........ 34

Figure 3.21: Halloran Summit Road, Nipton, CA to Barstow, CA real world driving cycle [56] 34

Figure 3.22: Bruceton Mills to Sabraton on I 68, WV driving cycle [56] .................................... 35

Figure 3.23: Sabraton to Bruceton Mills on I 68, WV driving cycle [56] .................................... 35

Figure 3.24: Scheme of relationship between distance and elevation of the road ......................... 37

Figure 3.25: Repeated Cruise cycle with 2\% maximum grade and $10 \mathrm{~km}$ period........................ 39 
Figure 3.26: Repeated Cruise cycle with 4\% maximum grade and $5 \mathrm{~km}$ period....................... 39

Figure 3.27: Part of repeated Cruise cycle with 2\% maximum grade and $10 \mathrm{~km}$ period ............ 40

Figure 3.28: Repeated HDUDDS cycle with 3\% maximum grade and $5 \mathrm{~km}$ period ................. 40

Figure 3.29: Part of repeated HDUDDS cycle with 3\% maximum grade and $5 \mathrm{~km}$ period ........ 41

Figure 3.30: Combined Cruise, CSHVR, Transient, and HDT65 cycle with 2\% maximum grade and $10 \mathrm{~km}$ period

Figure 4.1: Subsystem control strategy for a direct parallel pre-transmission HEV to calculate engine torque demand [54]

Figure 4.2: Diagram of the proposed looking-ahead subsystem control strategy for a direct parallel pre transmission HEV to manage the battery SOC 44

Figure 4.3: Scheme of looking-ahead strategy for forthcoming traffic conditions. 47

Figure 4.4: Energy storage system efficiency and power on heavy duty transient driving cycle. 49

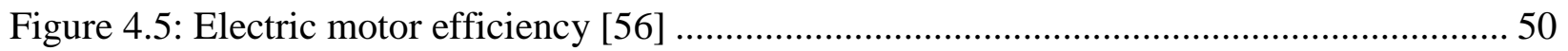

Figure 4.6: Electric motor efficiency map for $150 \mathrm{~kW}$ motor power [56] ............................. 50

Figure 4.7: Charging and discharging time for different energy storage system sizes 52

Figure 4.8: Negative road grade to start using brake system relative to vehicle speed and weight for heavy duty vehicle 53

Figure 4.9: Negative road grade to start using brake system relative to vehicle speed and weight for light duty vehicle

Figure 4.10: Power demand at wheels for truck $(25,000 \mathrm{~kg})$ at different negative road grade .... 55 Figure 4.11: Power demand at wheels for light duty vehicle $(1000 \mathrm{~kg})$ at different negative road grade

Figure 4.12: Negative road grade when driver needs to start depressing brake with different rolling resistance coefficient values 57

Figure 4.13: Negative road grade when driver needs to start depressing brake with different air drag coefficient values

Figure 4.14: Negative road grade when driver needs to start depressing brake with different rolling resistance and air drag coefficients values

Figure 4.15: Negative road grade change due to improved rolling resistance and air drag coefficients. 
Figure 4.16: Power available to capture descending hill (-3\%) for heavy duty vehicle $(25,000 \mathrm{~kg})$ 60

Figure 4.17: Impact of improving the rolling resistance and the air drag coefficients on heavy duty vehicle $(25,000 \mathrm{~kg})$ descending hills $(-3 \%)$. 60

Figure 5.1: Impact of the ITS on FC, battery SOC, engine energy losses and electric motor energy losses for going downhill and highway driving cycle.

Figure 5.2: Impact of the ITS on FC, battery SOC, engine energy losses and electric motor energy losses for going uphill and highway driving cycle 65 Figure 5.3: Impact of looking-ahead on the battery SOC for descending and ascending hills and highway driving cycles 66

Figure 5.4: Impact of looking-ahead on the battery SOC for different up-downhill grades and highway driving cycles

Figure 5.5: Sophisticated algorithm for different up-downhill grades and highway driving cycle

Figure 5.6: Battery SOC variation on different road grade degrees with simple and sophisticated looking-ahead propulsion controller.

Figure 5.7: Engine energy losses with No ITS, simple ITS, and advanced ITS control strategy for the different road grade degrees with highway driving cycle. 69 Figure 5.8: FC on uphill highway driving cycle for parallel HEV (150 kW_10kWh) at $50 \%$ load 70

Figure 5.9: FC rate on down-uphill highway driving cycle for parallel HEV (150 kW_10kWh) at $50 \%$ load 71

Figure 5.10: Fuel economy for different up-downhill terrains for conventional model and parallel HEV with different equipment sizes with $50 \%$ load. 72

Figure 5.11: Fuel economy change for a conventional and parallel HEV with No ITS control strategy model 73

Figure 5.12: Fuel economy changing for a conventional and parallel HEV with ITS control strategy model 74

Figure 5.13: Fuel economy changing for a conventional and parallel HEV with ITS once and No ITS second control strategy model 74 
Figure 5.14: Fuel economy change for a parallel HEV with ITS and No ITS control strategy

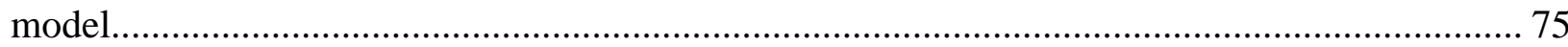

Figure 5.15: Fuel hot map for parallel HEV model (50\% load) with looking-ahead strategy .... 76 Figure 5.16: Fuel hot map for parallel HEV model (50\% load) without looking-ahead strategy 76 Figure 5.17: Regenerative braking recovered at battery for parallel HEV model (100 kW_5kWh) with $50 \%$ load 77

Figure 5.18: Regenerative braking recovered at battery change for parallel HEV model (100 kW_5kWh) with $50 \%$ load 77

Figure 5.19: ESS activity for parallel HEV model with looking-ahead strategy on down-uphill constant speed driving cycle. 78 Figure 5.20: ESS activity for parallel HEV model without looking-ahead strategy on down-uphill constant speed driving cycle. 78

Figure 5.21: Fuel economy for different real world cycles for parallel HEV model (GVWR $26,000 \mathrm{~kg})$ 79

Figure 5.22: Fuel economy change for different scenarios on BM2Sab cycle as a result of the looking-ahead strategy

Figure 5.23: Fuel economy change for different scenarios on Sab2BM cycle as a result of the looking-ahead strategy 80

Figure 5.24: Fuel economy change for different scenarios on Clm2Ind cycle as a result of the looking-ahead strategy

Figure 5.25: Fuel economy change for different scenarios on Las2Hall cycle as a result of the looking-ahead strategy

Figure 5.26: Fuel economy change for different scenarios on Hall2Bar cycle as a result of the looking-ahead strategy

Figure 5.27: Cumulative FC for parallel HEV with and without looking-ahead strategy on the real world driving cycle Sab2BM.

Figure 5.28: Cumulative FC for parallel HEV with and without looking-ahead strategy on the real world driving cycle BM2Sab

Figure 5.29: The energy recovered at battery using the brake system only on different real world driving cycles. 84 
Figure 5.30: Impact of looking-ahead strategy on the amount of free energy recovered at battery for different real world driving cycles 84

Figure 5.31: Fuel economy for different scenarios on repeated Cruise driving cycle with different road grade and terrain types...... 86 Figure 5.32: Fuel economy change for different scenarios on repeated Cruise driving cycle with different road grade and terrain types 86 Figure 5.33: Fuel economy for repeated HDUDDS cycle for a parallel HEV (150 kW \& $10 \mathrm{kWh})$ model with looking-ahead strategy and without it. 87 Figure 5.34: Fuel economy change for repeated HDUDDS cycle for a parallel HEV (150 kW \& $10 \mathrm{kWh}$ ) model with looking-ahead strategy and without it. 87

Figure 5.35: Fuel economy for combined driving cycle for a parallel HEV (150 kW \& $10 \mathrm{kWh})$ model with looking-ahead strategy and without it. 88 Figure 5.36: Fuel economy change for combined driving cycle for a parallel HEV (150 kW \& 10 $\mathrm{kWh}$ ) model with looking-ahead strategy and without it...... 88

Figure 5.37: The battery capacity for charging and discharging with and without looking-ahead strategy.

Figure 5.38: Energy storage system effectiveness on ascending hill-highway driving cycle with ITS and No ITS strategy 90

Figure 5.39: Energy storage system effectiveness on descending hill-highway driving cycle with ITS and No ITS strategy. 91

Figure 5.40: Energy storage system effectiveness on descending-ascending hill-highway driving cycle with ITS and No ITS strategy. 91

Figure 5.41: Vehicle speed for different models of $100 \%$ full load truck on down-uphill cycle .93 Figure 5.42: Engine power for the different vehicle models with $100 \%$ full load on down-uphill cycle

Figure 5.43: Battery SOC for HEV model with and without looking-ahead strategy with 100\% full load on down-uphill cycle 94 Figure 5.44: Electric motor power for HEV model with and without looking-ahead strategy with $100 \%$ full load on down-uphill cycle. 94

Figure 5.45: Impact of looking-ahead control strategy on maintaining vehicle speed on BM2Sab cycle 95 
Figure 5.46: Impact of looking-ahead control strategy on maintaining vehicle speed (GVWR $36,000 \mathrm{~kg}$ ) on Clm2Ind cycle

Figure 5.47: Impact of looking-ahead control strategy on maintaining vehicle speed (GVWR $36,000 \mathrm{~kg}$ ) on Hall2Bar cycle..... 96

Figure 5.48: Impact of looking-ahead control strategy on maintaining vehicle (GVWR 36,000 $\mathrm{kg})$ speed on Las2Hall cycle.....

Figure 5.49: Impact of looking-ahead control strategy on maintaining vehicle (GVWR 36,000 $\mathrm{kg}$ ) speed on Sab2BM cycle ..... 97

Figure 5.50: Vehicle speed for model (GVWR 36,000 kg) with conventional engine and with smaller engine relative to HEV model with ITS

Figure 5.51: Engine power for a conventional (smaller engine) and HEV (GVWR $36000 \mathrm{~kg}$ ) with ITS and No ITS

Figure 5.52: Motor power for a conventional (smaller engine) and HEV (GVWR 36,000 kg) with ITS and No ITS 100

Figure 5.53: Battery SOC for the HEV (GVWR 36,000 kg) with ITS and No ITS 100

Figure 5.54: Cumulative fuel consumption verses distance traveled for three vehicles modeled on Sab2BM real world driving cycle 101

Figure 5.55: Distance finished by different models on constant speed with different road grade route 102

Figure 5.56: Distance finished by different models on real world driving cycle Sab2BM route 102 Figure 5.57: Vehicle speed variation due to engine downsize for vehicle model (GVWR = 36,000 $\mathrm{kg}$ ) on Sab2BM route 103

Figure 5.58: Vehicle speed variation due to engine downsize for vehicle model (GVWR = 36,000 $\mathrm{kg})$ on $\mathrm{BM} 2 \mathrm{Sab}$ route. 103

Figure 5.59: Vehicle speed variation due to engine downsize for vehicle model (GVWR = 36,000 $\mathrm{kg}$ ) on Clmb2Ind route 104

Figure 5.60: Vehicle speed variation due to engine downsize for vehicle model (GVWR = 36,000 $\mathrm{kg}$ ) on Las2Hall route 104

Figure 5.61: Vehicle speed variation due to engine downsize for vehicle model (GVWR = 36,000 $\mathrm{kg}$ ) on Hall2Bar route 105 
Figure 5.62: Engine hot efficiency map for parallel HEV model with full size engine $(410 \mathrm{~kW})$ at Las2Hall driving cycle 106

Figure 5.63: Engine hot efficiency map for parallel HEV model with smaller engine size (310 $\mathrm{kW})$ at Las2Hall driving cycle 106

Figure 5.64: Engine hot efficiency map for parallel HEV model with full engine size $(410 \mathrm{~kW})$ at Sab2BM driving cycle 107

Figure 5.65: Engine hot efficiency map for parallel HEV model with smaller engine size (310 $\mathrm{kW})$ at Sab2BM driving cycle 107

Figure 5.66: Engine hot efficiency map for parallel HEV model with full size engine (410 kW) at BM2Sab driving cycle 108

Figure 5.67: Engine hot efficiency map for parallel HEV model with smaller size engine (310 $\mathrm{kW})$ at $\mathrm{BM} 2 \mathrm{Sab}$ driving cycle 108

Figure 5.68: ESS activity on Sab2BM driving cycle with full engine size (410 kW). 109

Figure 5.69: ESS activity on Sab2BM driving cycle with smaller engine size $(310 \mathrm{~kW}) \ldots \ldots \ldots 109$

Figure 5.70: Fuel economy for parallel HEV_150kW_10kWh_ITS .

Figure 5.71: FE change for parallel HEV_150kW_10kWh_ITS model (GVWR = 36,000 kg)

with full size engine and smaller engine with looking-ahead control strategy

Figure 5.72: Engine efficiency for parallel HEV_150kW_10kWh_ITS model (GVWR = 36,000 $\mathrm{kg}$ ) with full size engine and smaller engine with looking-ahead control strategy 111 Figure 5.73: Engine efficiency change for parallel HEV_150kW_10kWh_ITS model (GVWR = $36,000 \mathrm{~kg}$ ) with full size engine and smaller engine with looking-ahead control strategy 112 Figure 5.74: Linear regression between simulated HEV with looking-ahead and target vehicle speed for BM2Sab driving cycle.

Figure 5.75: Linear regression between simulated HEV with looking-ahead and target vehicle speed for Sab2BM driving cycle.

Figure 5.76: Linear regression between simulated HEV with looking-ahead and target vehicle speed for CLMB2IND driving cycle 114

Figure 5.77: Linear regression between simulated HEV with looking-ahead and target vehicle speed for Hall2Bar driving cycle 
Figure 5.78: Battery SOC and total energy for parallel HEV model with full size battery and no ITS in comparison to parallel HEV model with smaller battery size and ITS at Las2Hall driving cycle

Figure 5.79: Fuel economy for parallel HEV model $(\mathrm{GVWR}=36,000 \mathrm{~kg})$ with $10 \mathrm{kWh}$ battery energy size (without looking-ahead strategy) and $5 \mathrm{kWh}$ battery energy size (with looking-ahead strategy) 118

Figure 5.80: Fuel economy change for parallel HEV model (GVWR = 36,000 kg) with $10 \mathrm{kWh}$ battery energy size (without looking-ahead strategy) and $5 \mathrm{kWh}$ battery energy size (with looking-ahead strategy)

Figure 5.81: Braking energy recovered at battery for parallel $\mathrm{HEV}$ model $(\mathrm{GVWR}=36,000 \mathrm{~kg}$ ) with $10 \mathrm{kWh}$ battery energy size (without looking-ahead strategy) and $5 \mathrm{kWh}$ battery energy size (with looking-ahead strategy)

Figure 5.82: Percent regenerative braking battery for parallel HEV model (GVWR = 36,000 kg) with $10 \mathrm{kWh}$ battery energy size (without looking-ahead strategy) and $5 \mathrm{kWh}$ battery energy size (with looking-ahead strategy)

Figure A.1: Speed simulation results goodness for a conventional model at Clmb2Ind real world driving cycle.

Figure A.2: Speed simulation results goodness for a conventional model at Hall2Bar real world driving cycle.

Figure A.3: Speed simulation results goodness for a HEV with NoITS model at BM2Sab real world driving cycle

Figure A.4: Speed simulation results goodness for a HEV with NoITS model at Clmb2IND real world driving cycle 130

Figure A.5: Speed simulation results goodness for a HEV with NoITS model at Hall2Bar real world driving cycle

Figure A.6: Speed simulation results goodness for a HEV with NoITS model at Las2Hall real world driving cycle

Figure A.7: Speed simulation results goodness for a HEV with NoITS model at Sab2BM real world driving cycle

Figure A.8: Speed simulation results goodness for a HEV with ITS model at BM2Sab real world driving cycle. 
Figure A.9: Speed simulation results goodness for a HEV with ITS model at Clmb2Ind real world driving cycle

Figure A.10: Speed simulation results goodness for a HEV with ITS model at Hall2Bar real world driving cycle

Figure A.11: Speed simulation results goodness for a HEV with ITS model at Las2Bar real world driving cycle.

Figure A.12: Speed simulation results goodness for a HEV with ITS model at Sab2BM real world driving cycle

Figure B.1: Fuel economy for parallel HEV relative to conventional vehicle over different terrain types 135

Figure B.2: Fuel economy saving for parallel HEV relative to conventional vehicle over different terrain types 136

Figure B.3: Engine efficiency change for parallel HEV relative to conventional vehicle over different terrain types. 136 Figure B.4: Regenerative braking energy (\%) for parallel HEV relative to conventional vehicle over different terrain types

Figure B.5: Regenerative braking energy (Wh /mile) for parallel HEV relative to conventional vehicle over different terrain types 137 Figure C.1: Forward and reverse energy losses from the parallel HEV model equipment without looking-ahead strategy. 138 Figure C.2: Forward and reverse energy losses from the parallel HEV model equipment without looking-ahead strategy. 138 Figure C.3: Equipment efficiencies and energy balance for parallel HEV model without lookingahead strategy. 139 Figure C.4: Equipment efficiencies and energy balance for parallel HEV model with lookingahead strategy. 


\section{List of Tables}

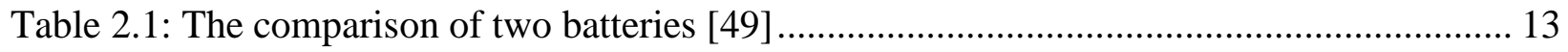

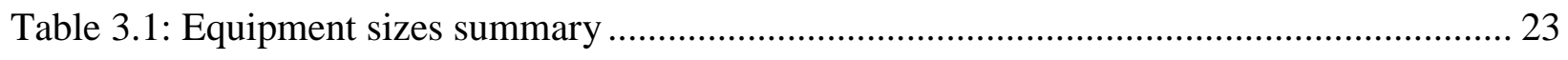

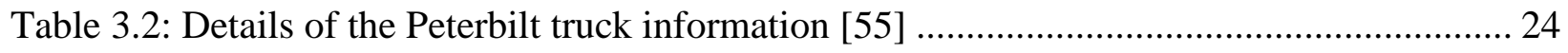

Table 3.3: Validation results of peterbilt truck ................................................................ 26

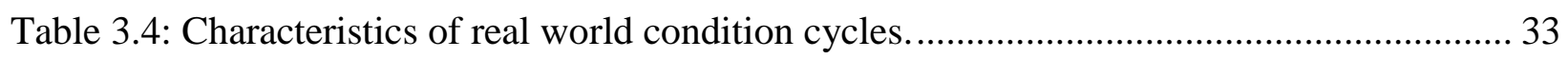

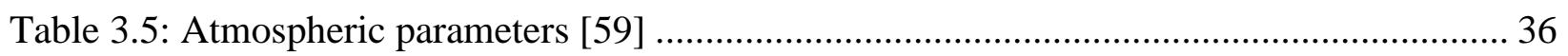

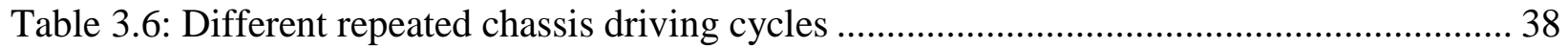

Table 3.7: Road grades and period generated to match with chassis cycles............................. 38

Table 5.1: Evaluation of the simulation results goodness.................................................... 115 


\section{Nomenclature and Abbreviations}

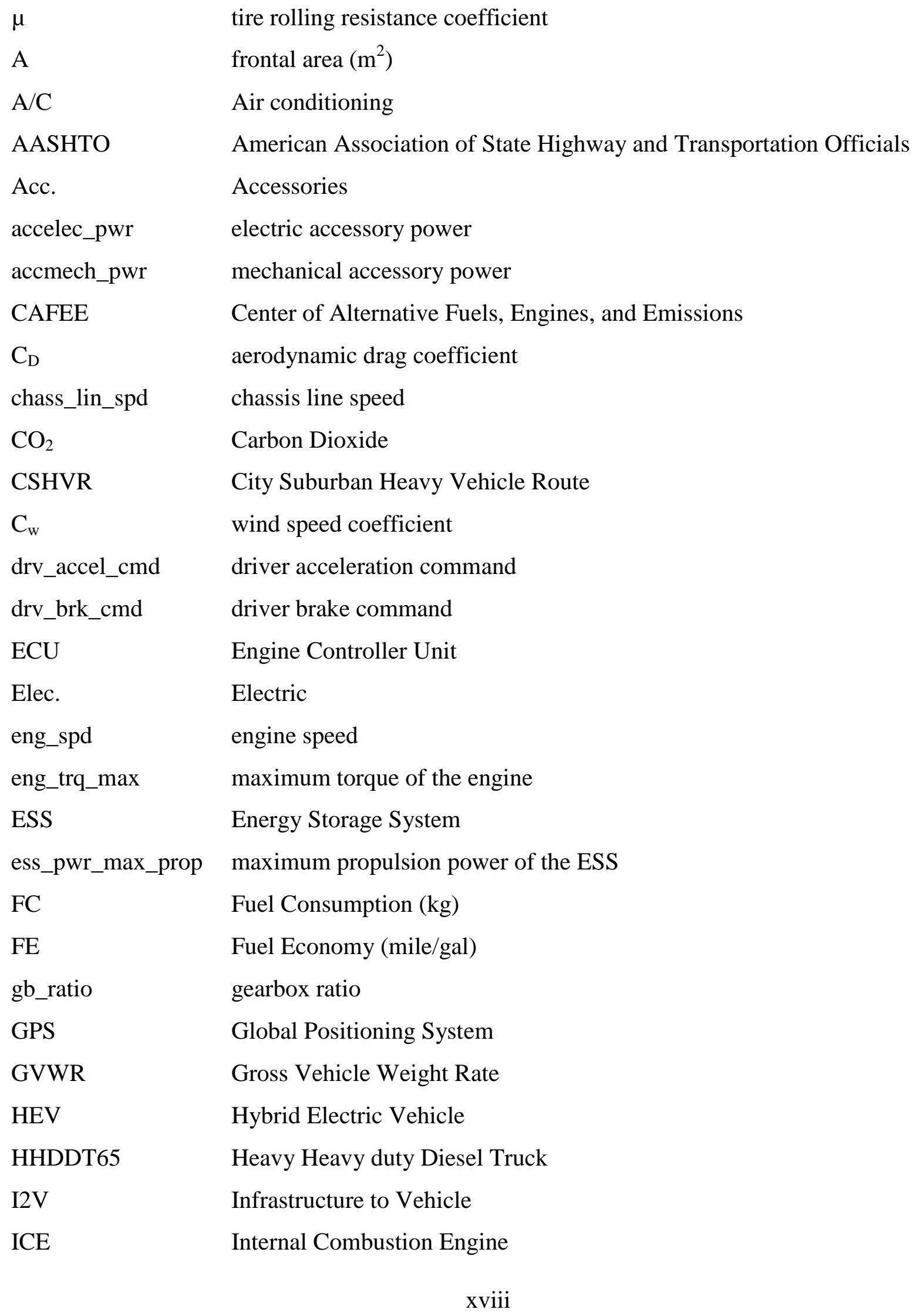




\begin{tabular}{|c|c|}
\hline ITS & Intelligent Transportation System \\
\hline Li-ion & Lithium ion \\
\hline $\mathrm{m}$ & mass $(\mathrm{kg})$ \\
\hline MEMS & Mobile Emission Measurement System \\
\hline mot_spd & electric motor speed \\
\hline mot_trq_max_regen & maximum torque of the electric motor \\
\hline $\mathrm{Ni}-\mathrm{MH}$ & Nickle-Metal Hydride \\
\hline$\Theta$ & road grade (radian) \\
\hline OCTA & Orange County Transit Authority \\
\hline PEMS & Portable Emission Measurement System \\
\hline PSAT & Powertrain System Analysis Toolkit \\
\hline $\mathrm{R}^{2}$ & square of correlation \\
\hline $\mathrm{RE}$ & Relative Error \\
\hline RMSE & Root Mean Square Error \\
\hline Sc1 & Scenario 1 \\
\hline $\mathrm{Sc} 2$ & Scenario 2 \\
\hline $\mathrm{Sc} 3$ & Scenario 3 \\
\hline Sc4 & Scenario 4 \\
\hline $\operatorname{Sc} 5$ & Scenario 5 \\
\hline $\mathrm{SOC}$ & State of Charge \\
\hline UDDS & Urban Dynamometer Driving Schedule \\
\hline $\mathrm{v}$ & vehicle speed $(\mathrm{m} / \mathrm{sec})$ \\
\hline VDP & Vehicle Development Process \\
\hline VPC & Vehicle Propulsion System \\
\hline whl_spd & wheel speed \\
\hline WVU & West Virginia University \\
\hline$\rho$ & air density $\left(\mathrm{kg} / \mathrm{m}^{3}\right)$ \\
\hline
\end{tabular}




\section{Chapter 1 : Introduction}

\subsection{Background}

About one-third of the cost of a heavy diesel truck's life cycle comes from fuel expenses [1]. This cost, along with salaries, is the largest share of the total cost to the owners of trucks. Moreover, heavy duty vehicle engine design modifications were driven mainly by emissions regulations. According to government and industry statistics, heavy duty trucks account for about $20 \%$ of carbon dioxide $\left(\mathrm{CO}_{2}\right)$ emissions from all transportation sources and the heavy duty vehicle is the fastest expanding sector [2]. In September 2011, the National Highway Traffic Safety Administration and U.S. Environmental Protection Agency (EPA) promulgated the firstever federal regulations mandating fuel economy improvements for heavy duty commercial vehicles [1]. Therefore, increased worry about the fuel economy and emissions from vehicles has directed interest in the use of hybrid electric powertrains and the start of several production vehicles in both heavy duty and light duty applications [2]. Hybrid electric vehicles (HEVs) use a combination of electric motor and another power source such as an internal combustion engine IC engine or fuel cell [3-5]. While these vehicles show great potential for use in a wide variety of driving situations, the optimization of components and control strategies is somewhat complex. One of the potential optimization methods is the looking-ahead strategy. Therefore, this study focuses on the potential impact of a looking-ahead strategy on parallel HEVs. Looking-ahead seeks to estimate the forthcoming power events in advance and manage the alternative power source depending on this advanced knowledge.

The powertrain of a parallel hybrid electric vehicle has an electric motor, which works as an alternative power source, in addition to an IC engine. The main energy source for a parallel hybrid electric heavy duty vehicle is the fuel such as diesel or natural gas, unless it is a parallel plug-in HEV. A parallel plug-in HEV uses the grid electric source as another alternative energy source to charge the battery. The secondary power source is the electric power which is stored in an Energy Storage System (ESS) [6]. Energy stored in the ESS comes either from the engine or from captured energy while using the regenerative brake system [6-8]. Several studies have proven that the benefits from parallel HEVs are limited on flat highway and that is due to the limited use of the regenerative brake system $[9,10]$. On the other hand, these assessments might not apply to heavy duty HEVs because the heavy duty vehicle's engine runs near the peak load 
more than the engine of a light duty vehicle, and the heavy duty vehicle has large inertia load in comparison to light duty vehicles [11]. A significant inertia load for a heavy duty vehicle is due to its relatively large mass. It is expected that a heavy duty vehicle needs additional power for acceleration or ascending hills. Also, it is expected that the brake system for a heavy duty truck will be used much more than a light duty vehicle to compromise speed or slow down [12]. Since going uphill and downhill terrains may represent the major reason to encourage using hybridization systems for a heavy duty vehicle, they have attracted substantial interest in recent years due to their potential for decreasing fuel consumption [13]. Furthermore, a parallel hybrid electric vehicle architecture is expected to improve energy conversion efficiency [13].

The real time data on traffic flow and road conditions are available through vehicle-to-vehicle (V2V) and vehicle-to-infrastructure communication (V2I) in the intelligent transportation systems (ITS). The data expected from ITS can be used in different ways to adjust the tuning of the energy management controller based on the predicted the future driving profile, and in generating statistical information to be used in stochastic dynamic programming. The information available from ITS technology can contain large quantities of data on road conditions, traffic flow, and weather. The knowledge of some information can be more important than others; it is necessary to find the impact of the data on the energy management and to identify the crucial pieces of information necessary to improve the energy management performance [15].

Predictive controller strategies for a HEV have been analyzed in several studies [14-16]. Different simulations and measurements have proven the potential benefits from knowing the forthcoming traffic conditions [17, 18]. The knowledge of traffic conditions ahead can be available by communicating with infrastructure and collecting information [19]. On the other hand, most of these studies were for light-duty HEVs to manage the hybrid power system in advance [20]. The vehicle propulsion control improvement showed a strong impact on fuel economy for a hybrid electric vehicle [21]. This study suggested an algorithm which may be more proper for heavy duty HEVs configuration and effective at the same time. The ultimate goal of this proposed strategy was to manage ESS State of Charge (SOC) for opportunistic regenerative braking. 
Since the Global Positioning System (GPS) is already established, the intelligent transportation systems (ITS) communication with the vehicle does not need expensive equipment [14, 22]. Looking-ahead strategy is to know the forthcoming traffic condition regarding congestion and road grade to prepare the system in advance. Preparing the alternative power source for a heavy duty HEV in advance is accomplished by ensuring a maximum capacity in the ESS for forthcoming expected power from using the brake system or maximum needed power (limited by ESS capacity) to assist the engine for the forthcoming additional power demand.

In this work, a parallel direct pre-transmission hybrid electric vehicle configuration with and without looking-ahead strategy has been investigated under three different motor power capacities $(100,150,200 \mathrm{~kW})$ and three different battery energy capacities $(5,10,15 \mathrm{kWh})$ to study the impact of hybridization equipment sizes on fuel economy, maintaining adequate power, and downsizing powertrain equipment opportunity. The vehicle model used in this study was a class 8 truck with engine power of $410 \mathrm{~kW}$. Different chassis driving cycles with different road grades in parallel with five real world highway driving cycles have been used to show the impact of the looking-ahead control strategy on managing the energy storage system and fuel economy improvement.

\subsection{Hypothesis and objectives}

A hybrid electric vehicle is expected to increase fuel economy and offer an alternative power source for the vehicle. Driving the vehicle on a known route with known traffic conditions in advance may give a privilege to the vehicle propulsion controller to take advantage of lookingahead information in order to calculate the power demand ahead and manage the battery SOC in opportunistic regenerative braking based on that demand. In other words, the Vehicle Propulsion Controller (VPC), based on information ahead, will use the road load equation to estimate the power demand ahead, and the VPC works based on that calculation. The global objective of this research is to study the possible benefits from hybrid electric vehicle with looking-ahead control strategy. The plan is to modify the VPC strategy in the parallel HEV model using Autonomie as simulation software [24], and enable it to deal with the intelligent transportation system (ITS) information regarding road grade and vehicle speed ahead. For all objectives several real world and chassis driving cycles for heavy duty vehicles will be used. 
The major objectives of this research are as follows:

1- Study the possible impact of ITS information for the forthcoming traffic condition regarding route elevation and congestion on fuel consumption for a direct parallel pre-transmission HEV.

2- Explore the contribution of ITS information for the route ahead on improving battery pack effectiveness as an alternative power system.

3- Study the possible impact of route knowledge ahead regarding elevation and traffic conditions on improving engine power management by maintaining adequate power.

4- Explore the detail depth needed from ITS information to estimate the forthcoming negative or positive power demand of the truck.

\subsection{Technical approaches}

This study investigated the contribution of knowledge ahead regarding route elevation and traffic conditions in improving fuel efficiency for heavy duty direct parallel HEVs. A time window opened in the future for a specific period of time to estimate the power demand. The road load equation was used to calculate the power demand from the forthcoming average speed and road grade. The forthcoming power demand estimation helped the vehicle propulsion controller (VPC) manage the battery SOC for opportunistic regeneration. If the forthcoming power demand is positive and it will surpass the engine peak power, then the battery will be charged. If the forthcoming power demand is negative, which means that the brake system will be in use, then the battery SOC will be depleted to ensure enough capacity to capture upcoming free energy. A time window opened to enable the VPC to detect the forthcoming power events. Five scenarios were studied. In the first scenario, the ITS information ahead was the forthcoming route elevation with $\pm 1.5 \%$ road grade resolution, vehicle speed, and opening one window each time. In the second scenario, the ITS information ahead was the forthcoming route elevation, speed limit, and opening one window each time. In the third scenario, the ITS information was the route elevation, speed ahead with $\pm 5 \mathrm{mph}$ resolution, and opening one window each time. In the fourth scenario, the ITS information was the route elevation, speed ahead with $\pm 10 \mathrm{mph}$ resolution, and opening one window each time. In the fifth scenario, the ITS information ahead was the route elevation, speed limit, actual speed, and stop detection. In the fifth scenario a two time window were opened. One window for short duration ahead, such as 1 minute ahead was 
used, to detect an upcoming stop. A second time window for a longer duration such as 3, 5, and 7 minutes ahead, route elevation and anticipated speed. These time windows ahead were depending mainly on the battery and the electric motor sizes. The time needed to charge or discharge the ESS was calculated based on the battery and electric motor sizes with considering all equipment efficiencies, and the long duration window time was opening based on that calculation.

\subsection{Contributions}

Parallel hybrid electric heavy duty vehicles have attracted increasing interest to provide improved fuel efficiency and further development, and optimization of the technology is still needed. A hybrid control system that has prior knowledge of power demands and regeneration opportunities has the potential to offer better performance than one that has no knowledge of the road ahead. Real world traffic situations and route information constitute can support a lookingahead control strategy that provides for better use of the on-board energy storage system.

The primary contribution of this study is developing and modifying of the vehicle propulsion controller within a model to enable a looking-ahead strategy for the forthcoming negative or positive power demands. The vehicle propulsion controller for the parallel hybrid electric heavy duty vehicle model developed in this research added management of the energy storage system for opportunistic regeneration. The control scheme guaranteed motor energy to assist the conventional engine drivetrain when the vehicle was ascending hills. It also sought to optimize the capture any forthcoming regenerative energy that was gained when depressing the brake pedal, by depleting the charge in the energy storage system before the braking event. The novelty of this study included the development of the vehicle propulsion controller for the parallel hybrid electric heavy duty vehicle model. Parallel hybrid electric heavy duty vehicle model with and without looking-ahead strategy have been built to establish the relative expected benefits from the looking-ahead control strategy.

A conventional heavy duty vehicle model was used as well to study the impact of the parallel hybrid electric heavy duty vehicle with and without looking-ahead strategy in comparison to it. A further contribution was that the advanced control benefits were demonstrated both with 
idealized grades and with real-world test schedules that incorporated speed and grade. Computed results showed that the benefit can vary with the type of test schedule, and that variables within the looking-ahead hybrid control model influenced the fuel economy of the truck. A further contribution is the clear demonstration that road grade information will be very important in test schedules for parallel hybrid electric trucks, with or without a looking-ahead strategy.

Results suggest that there is an opportunity to downsize the engine in a parallel hybrid electric vehicle model with looking-ahead strategy while maintaining adequate power and improving fuel economy. The model, produced from this study on the Autonomie platform, is available for future users to assess performance of different vehicle types operating with different loads over any chosen route. A consequence of this research, and related research by others, will be the development of vehicles that use increased intelligence and infrastructure-to-vehicle communication to improve fuel efficiency substantially. 


\section{Chapter 2 : Literature review}

\subsection{The road-load equation}

Total power required at the wheels was stated by the road-load equation [24-26] applied to a vehicle as:

$P_{t}=\left(m \frac{d v}{d t}+\frac{1}{2} C_{D} \rho A v^{2}+\mu \mathrm{mg}+\mathrm{mgZ}\right) v$

Equation 2.1

where, $\mathrm{m}$ is mass of the vehicle $(\mathrm{kg}), v$ is speed of the vehicle $(\mathrm{m} / \mathrm{sec})$ assuming no wind, A is frontal area of the vehicle $\left(\mathrm{m}^{2}\right), g$ is acceleration due to gravity $\left(9.81 \mathrm{~m} / \mathrm{sec}^{2}\right), C_{D}$ is aerodynamic drag coefficient, $\mu$ is tire rolling resistance coefficient, $\rho$ is air density, and $\mathrm{Z}$ is road gradient (\%). $\mathrm{P}_{\mathrm{t}}$ is the total power needed at the wheels and is stated in Watts (W). In this equation the first term was referred to as the inertia load, the second term as the wind drag, the third term as the rolling resistance, and the last term was the grade load. Summation of these loads resulted in the "total load or total power." This was also termed as 'tractive force' needed to propel the vehicle at any prescribed velocity and acceleration [27]. Moreover, there are other (accessories) power demands such as $\mathrm{A} / \mathrm{C}$, cooling and lights. The power demand for accessories did not account in this equation, but it accounted during the vehicle model simulation.

The aerodynamic drag in Equation 1 was defined as the force required to overcome the aerodynamic resistance to vehicle motion. The value of drag coefficient, $C_{D}$ for a chassis heavy duty vehicles used in the literature varied from 0.40 to 0.79 [27].

The rolling resistance was defined as the force required to move a vehicle over a surface and stated as $\mu^{*} \mathrm{~m}^{*} \mathrm{~g} * v$. The rolling resistance coefficient, $\mu$, was influenced by tire size and pressure, axle geometry, and the amount of load applied to the tires. The rolling resistance force was found to dominate the wind drag and inertia forces at low speed and steady operation while, at high speed operation, the wind drag dominated. The rolling resistance contributed about $20 \%$ of the total road load on the Orange County Transit Authority driving cycle (OCTA) [28]. A reduction in rolling resistance contributed to a decrease in FC. Previous research demonstrated that a $20 \%$ reduction in rolling resistance could yield as much as $2.5 \%$ reduction in FC [29]. The rolling 
resistance coefficient for a transit bus was found to vary from 0.005 when vehicle speed approached zero miles per hour (mph) to 0.011 when vehicle speed approached $56 \mathrm{mph}$ [30].

The road slope, $\mathrm{Z}$ was discussed as the slope of the road and it was stated as a percentage [31]. The numerical value of $\mathrm{Z}$ was considered as the vertical increase (+) or decrease (-) in meters (m) for every $100 \mathrm{~m}$ traveled [32]. The gradient resistance in Equation 1 was defined as the force that was needed to overcome the force that the vehicle's weight when climbing hills [31]. Moreover, this force could be negative when the vehicle descending hills.

\subsection{Vehicle propulsion energy demand}

The energy required to drive a vehicle comes from the power unit which is usually an internal combustion engine ICE. In an HEV an addition power source in the form of an electric motor and better supply additional propulsion power. The purpose of the transmission system is to enable the power unit to operate in its high efficiency zone at all vehicle speeds. Depending on the specifics of the transmission system, up to $20 \%$ of the energy supplied by the powertrain is lost to friction, i.e. efficiencies of above $80 \%$ are observed. The remaining energy (transferred to the wheels) is used to accelerate and elevate the vehicle, and to overcome driveline drag forces, rolling friction at the wheels, and air resistance [33].

\subsection{Hybrid electric vehicle}

A hybrid electric vehicle uses multiple sources of energy that can be autonomously or together used to propel the wheels. Normally, the energy sources include an electric motor with energy from the ESS, and a smaller than normal diesel engine that together provide the same power as a conventional heavy duty vehicle while reducing FC and emissions. However, these types of HEV have some challenges when inclining very long hills due to limited energy available in the ESS. 


\section{The clear benefits of a hybrid electric vehicle}

Ideal engine operating region: The engine has a region of highest efficiency on the torque-speed envelope. In an HEV (series or power split), the engine can operate more often in its region of highest efficiency. As the engine and traction model are not coupled directly (except the parallel HEV), the engine does not have to always follow the road load change. The engine can run in a less transient situation which results in significant reductions to FC and emissions [34].

Engine auto-stop: Fuel economy is very low when the engine operates at a low speed or idling status which is about $10 \%$ of the engine load or less. The engine is shut off when its speed is below a certain threshold thereby reducing FC and emissions [35].

Regenerative braking: A regenerative brake recovers the kinetic energy by converting it into electrical energy which can be stored in the ESS for future use. The kinetic energy is dissipated as heat in a conventional brake system. In HEVs, part of this energy could be recuperated depending on the duty cycle and aerodynamic drag and rolling resistance [34].

Improved drivability: The electric motor reacts faster than an internal combustion engine (ICE), so the torque required to reach the desired vehicle speed can be achieved faster than a conventional vehicle.

Electrical accessories: the mechanical accessories are replaced by electric systems which will save energy [35].

\section{Drawbacks of a hybrid electric vehicle}

The configuration is more complex than a conventional vehicle configuration.

The vehicle mass is heavier due to the addition of components such as ESS and electric motor.

The cost is increased due to the complexity of the energy control strategy and the additional components.

The reliability of the overall system is decreased due to the increased system complexity [6].

\subsection{Control systems for improving drivability}

It is important to explore in details to understanding of the drivability for both conventional and hybrid electric vehicles, since the main objective of this work is to evaluate the influences of hybrid electric vehicle control strategies on vehicle drivability. Consequently, this section reviews some of the dynamic control procedures used to improve drivability in conventional, 
electric and hybrid electric vehicles. Drivability is associated with the driver's sensitivity to the dynamic responses of the vehicle [36, 37]. Drivability is frequently evaluated by qualified drivers that evaluate the vehicle performance under precise dynamic conditions for example engine idling, launch, acceleration, cruise (low/high speed), pedal tip-in/tip-out, gear changes and engine on/off. The general evaluation of automobile drivability can be inefficient and is subject to human error. Lately, auto builders moved towards the use of special software sets to evaluate drivability in an independent way [38]. These software sets use methods (for example neural networks) that process a combination of sensors and data gained from several driving conditions.

\subsubsection{Empirical rule-based control strategy}

An empirical rule-based control strategy is based on "if-then-else" logic and uses the statistically best effectiveness map to attain the best fuel efficiency. The empirical rule is used to establish events which will be activated when firm conditions are met. The thermostatic control strategy is typical of a rule-based control approach. In a thermostatic control strategy, the state of charge is calculated as an event cause and the engine is turned on/off when the state of charge reaches the prescribed lower/upper bound. Rule-based strategies have been one of the most commonly used energy management approaches [37]. The rule-based control strategy is artless, real-time implementable and has fewer calculations in comparison to real time control strategy. The significance of the simplicity is that this strategy cannot promise the best FE all of the time. A significant volume of parameters need to be modified in order to find the maximum fuel efficiency point. A rule-based control strategy for a particular vehicle cannot be readily used for another vehicle [37].

\subsubsection{Real time control strategy}

This control strategy utilizes the equivalent consumption minimization strategy that is based on equivalent fuel flow rate to minimize the overall FC over a given driving cycle [39, 40]. For example, the equivalent fuel rate can be given by:

$\dot{m}_{\text {fuel_eq }}=\dot{m}_{\text {fuel }}-\beta * P_{\text {elect }}$

Equation 2.2 
where $\dot{\mathrm{m}}_{\text {fuel_eq }}$ is the equivalent fuel flow, $\dot{\mathrm{m}}_{\text {fuel }}$ is the real fuel, $\beta$ is the fuel equivalent factor with unit $g / J$, and $P_{\text {elect }}$ is the real electrical power. The equivalent energy from the energy storage system and the ICE is assessed by relating the cost of instant energy produced. If the ICE produces power more efficiently than the energy storage system, then the ICE is used as power to drive the vehicle, otherwise, the ESS is preferred.

The main advantage of a real time control strategy is the optimization of the entire system as a whole instead of just the engine only. This means that the general efficiency that can be realized at the energy path is either from the ESS to the wheels or from both of the ICE and ESS to the wheels.

In a real time control strategy, the types of operation can be considered as start, acceleration, cruise and deceleration as detailed:

Starting and acceleration: The energy storage system delivers power to propel the vehicle. The engine start/stop is depending on the existing electric energy.

Cruise: The engine is turned on and the engine power is used either to drive the wheels or charge the ESS.

Extra energy demand: The internal combustion engine and energy storage system are both connected to drive the vehicle. The electric motor provides extra torque to meet the wheel power request relative to engine power.

Decelerating or descending hills: The regenerative braking recuperates dynamic energy into a storable system for upcoming use. The engine during this phase can be either run at idle speed or be turned off.

The disadvantage of this method lies in the complexity of the system due to the connections of the drivetrain components (ICE, ESS and motor) [41].

\subsection{Look-ahead control for hybrid electric vehicle}

The look-ahead control primarily differs from instantaneously decision making on descending hills and ascending hills where the look-ahead control in general reduces or increases speed previous to the hill. A conventional cruise control fed with the forthcoming road grade information and managed to increase or decrease the speed. Slowing down prior to decline hills is automatically saving fuel. There is however no challenge in saving fuel by traveling slower, so 
if the vehicle is let to slow down at some point, the lost time must be gained at another point. Accelerating prior to up hills is one way which, at least for smaller hills, gives a higher velocity throughout the hill and will reduce the need for lower gears. Fuel economy improved about 3.5\% on a real world driving highway cycle for about $120 \mathrm{~km}$ route without an increase in the trip time. The controller performance has been observed as easy and natural by drivers and passengers that have joined in tests and examples [14].

\subsection{Backward-looking models (SIMPLEV, ADVISOR)}

The backward looking model works by assuming that the vehicle meets the desired speed cycle. The power demand and related wheel torque are calculated from the desired vehicle speed. The calculation travels backward through the vehicle drivetrain all the way to the engine to finally decide how each component should react to track the speed [42]. The backward looking model is relatively fast to run; however, since the backward looking models are static models which assume the vehicle speed cycle has already been met, transient effects are not accounted for realistically. Therefore, the backward looking approach cannot complete an accurate control strategy. ADVISOR, which was originally developed by NREL in 1994 and was further developed by AVL in 2004, is representative of a backward looking model. Other simulation programs, like Simple Electric Vehicle Simulation Software (SIMPLEV), also utilize backward looking approaches [43].

\subsection{Forward-looking model (Autonomie)}

Autonomie's composite modeling and simulation tool is a progressive model that was originally developed by the Argonne National Laboratory, under the supervision and with the contributions from Ford, General Motors and Chrysler. It works by imitating the appropriate driver for the development of the accelerator and brake pedal inputs in order to meet the target vehicle speed [44]. Autonomie is a flexible and reusable vehicle simulation package and automotive engineering tool that assesses a vehicle's FE and performance [44]. Operators can build a model car properly by selecting the engine, and component models, configuration file and control strategy and graphical user interface. This program allows researchers to improve the efficiency of simulation and improve the performance of the car, in addition to helping to reduce emissions and FC. Currently (2013), Autonomie has been used by more than 895 departments, including 
light duty auto makers, and heavy duty vehicle manufacturers, suppliers, oil companies and research bodies and universities [45].

\subsection{Energy storage system for hybrid electric vehicles}

\subsubsection{Battery pack}

It is important to choose the battery technology as the battery moves the hybrid electric vehicle in standings of price, reliability, mass and "greenhouse gas emissions" production [46, 47]. The battery technologies used widely in HEV include lead-acid, nickel-metal hydride (Ni-MH) and lithium ion (Li-ion) [48]. Lead-acid batteries were used in the initial period of HEVs, but most of the current vehicles operate with $\mathrm{Ni}-\mathrm{MH}$ and $\mathrm{Li}$-ion [46]. Table 2.1 compares and contrasts the two battery technologies [49]. The dominant battery type in the market for hybrid electric vehicle now is Li-ion [50].

Table 2.1: The comparison of two batteries [49]

\begin{tabular}{|l|l|l|}
\hline & Ni-MH & Li-ion \\
\hline Specific energy & $50 \mathrm{Wh} / \mathrm{Kg}$ to $65 \mathrm{Wh} / \mathrm{Kg}$ & $90 \mathrm{Wh} / \mathrm{Kg}$ - 95 Wh/Kg \\
\hline $\begin{array}{l}\text { Battery charging } \\
\text { and discharging } \\
\text { cycles }\end{array}$ & $200-300$ cycles & Above 1000 cycles \\
\hline $\begin{array}{l}\text { Cycle life of the } \\
\text { battery }\end{array}$ & $\begin{array}{l}\text { The calendar life of Ni-MH batteries } \\
\text { can be improved by full discharging } \\
\text { to prevent crystalline formation }\end{array}$ & $\begin{array}{l}\text { Li-ion batteries are subject to aging, } \\
\text { even if not in use }\end{array}$ \\
\hline $\begin{array}{l}\text { State of charge } \\
\text { voltage profile for this battery from } \\
80 \%-30 \%\end{array}$ & $\begin{array}{l}\text { The battery should not be fully } \\
\text { charged, high voltages stresses the } \\
\text { battery }\end{array}$ \\
\hline $\begin{array}{l}\text { Temperature } \\
\text { dependent } \\
\text { performance }\end{array}$ & $\begin{array}{l}\text { Shows unsatisfactory performance at } \\
\text { high or low temperatures }\end{array}$ & $\begin{array}{l}\text { The performance erodes } \\
\text { drastically at extreme } \\
\text { high/low temperatures }\end{array}$ \\
\hline
\end{tabular}




\subsubsection{Ultracapacitors}

An ultracapacitor is an electrochemical capacitor that has high energy density while having the same features of a usual capacitor [51]. The ultracapacitor contains two electrodes, which are separated by an insulator. This keeps current from flowing between the electrodes so that an electric potential is allowed to develop. The electrodes are normally built from a layer of activated carbon with metal foil. As the charge builds up on the electrodes, ions are attached to the surface of the activated carbon [52]. Ultracapacitors have clear advantages when compared with batteries, as described below:

The advantages of ultracapacitor are:

Long life span, without degrading the performance with use, which extends the life of a HEVs' power source.

Can be charged or discharged in a very short time, approximately ten times faster than a battery with the same weight, but unfortunately it can't maintain energy for a long time, and has a much lower energy storage capacity.

Low weight and volume.

Steady performance at temperatures as low as $-40{ }^{\circ} \mathrm{F}[52]$.

The hybrid electric vehicle with the ultracapacitors is better controlled at quick power transient due to its high power density, which enables an HEV with ultracapacitors to increase existing power during acceleration [52]. However, the ultracapacitors have some limitations such as variable voltage, which is negative in some cases and also they can't store energy for long time. Most of the existing ultracapacitor hybrids are designed for use in transit buses where their frequent stop-and-go duty cycles are conducive to the use of [52]. 


\section{Chapter 3 : The Model Overview}

\subsection{Autonomie overview}

Autonomie was constructed to be used as a tool through the several phases of model based design of the Vehicle Development Process (VDP) [53]. Model Based Design is a mathematically based graphical procedure for planning multifaceted control systems and is being used efficiently in many powertrains control, manufacturing, space, and motorized applications [53]. It delivers an effective method that encloses four important elements in the process: displaying a powertrain, generating and examining a controller for the powertrain, mimicking the powertrain and manager together, and encoding or ordering the controller [53]. Autonomie is the successor to the PSAT [45]. Figure (3.1) shows the general schematic of software running a simulation in Autonomie.

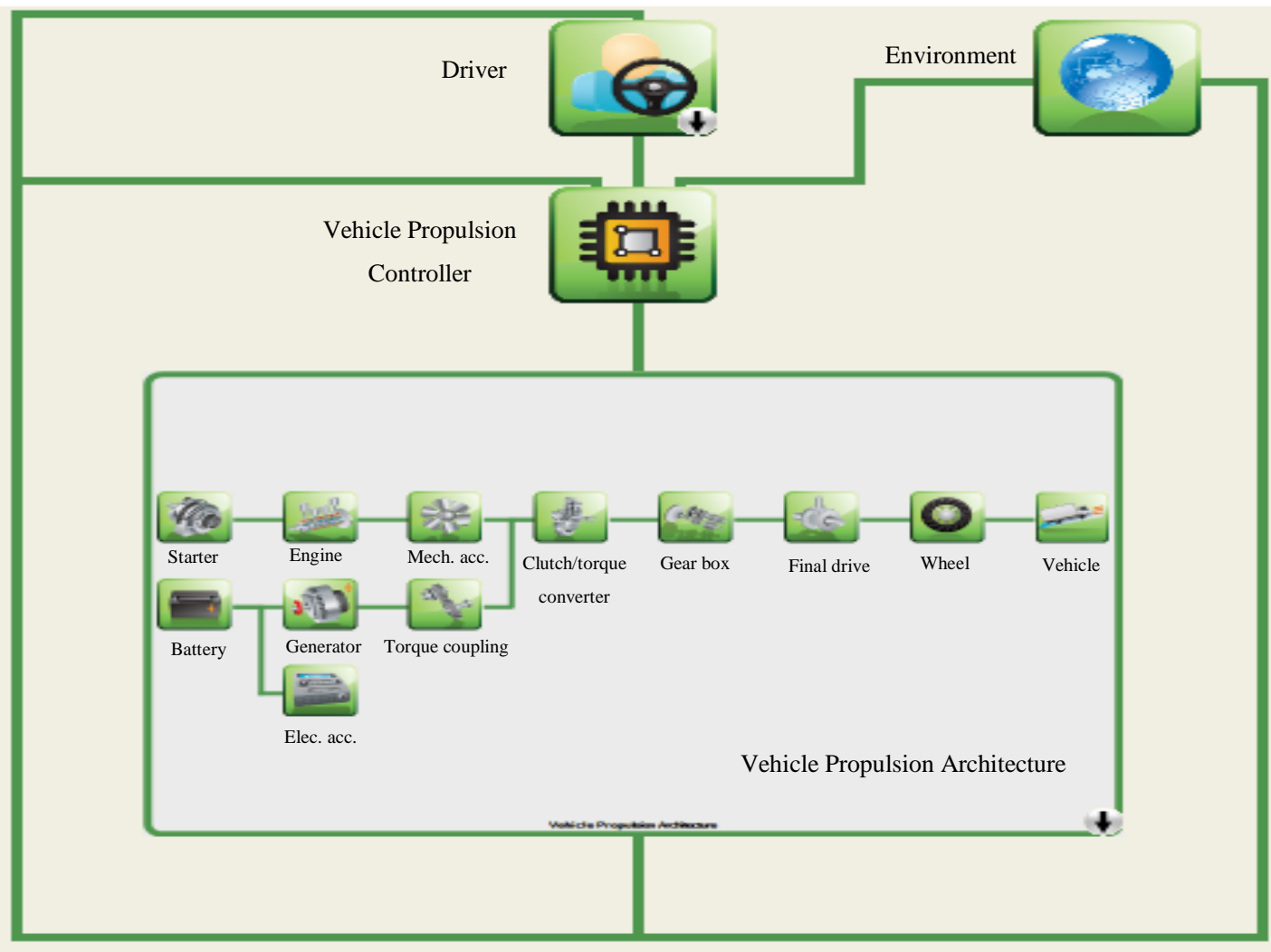

Figure 3.1: Schematic of conventional truck model in Autonomie [54] 


\subsection{Powertrain controller strategy for a direct parallel pre-transmission HEV}

The main components of parallel HEV are the electric motor/generator, energy storage system, and controller. Figure (3.2) shows the schematic of pre-transmission parallel HEV [23]. The control strategy for the VPC works by gathering all input parameters, which represent the demand and components limits, (driver acceleration demand, driver braking demand, engine torque limit, SOC) as shown in Figure (3.3) [54]. The control strategy for a direct parallel pretransmission HEV essentially works as follows: the main power source for the vehicle is the engine with the electric motor working as alternative power unit. The energy storage system is sustained at the target SOC and it will be charged if below that or use the power available if above the target energy level. The electric motor can assist the engine when it reaches the peak load as long as the battery has adequate energy. The electric motor can drive the vehicle alone and turn off the engine if the power demand is meeting the electric motor capability (it happens most at low speed). Figure (3.4) shows the subsystem control strategy for a direct parallel pretransmission HEV [54]. Each component in the HEV has a subsystem diagram to calculate energy demand and maximum power available from it. All abbreviations used in these subsystems were listed in the nomenclature and abbreviations list at the beginning of this dissertation.

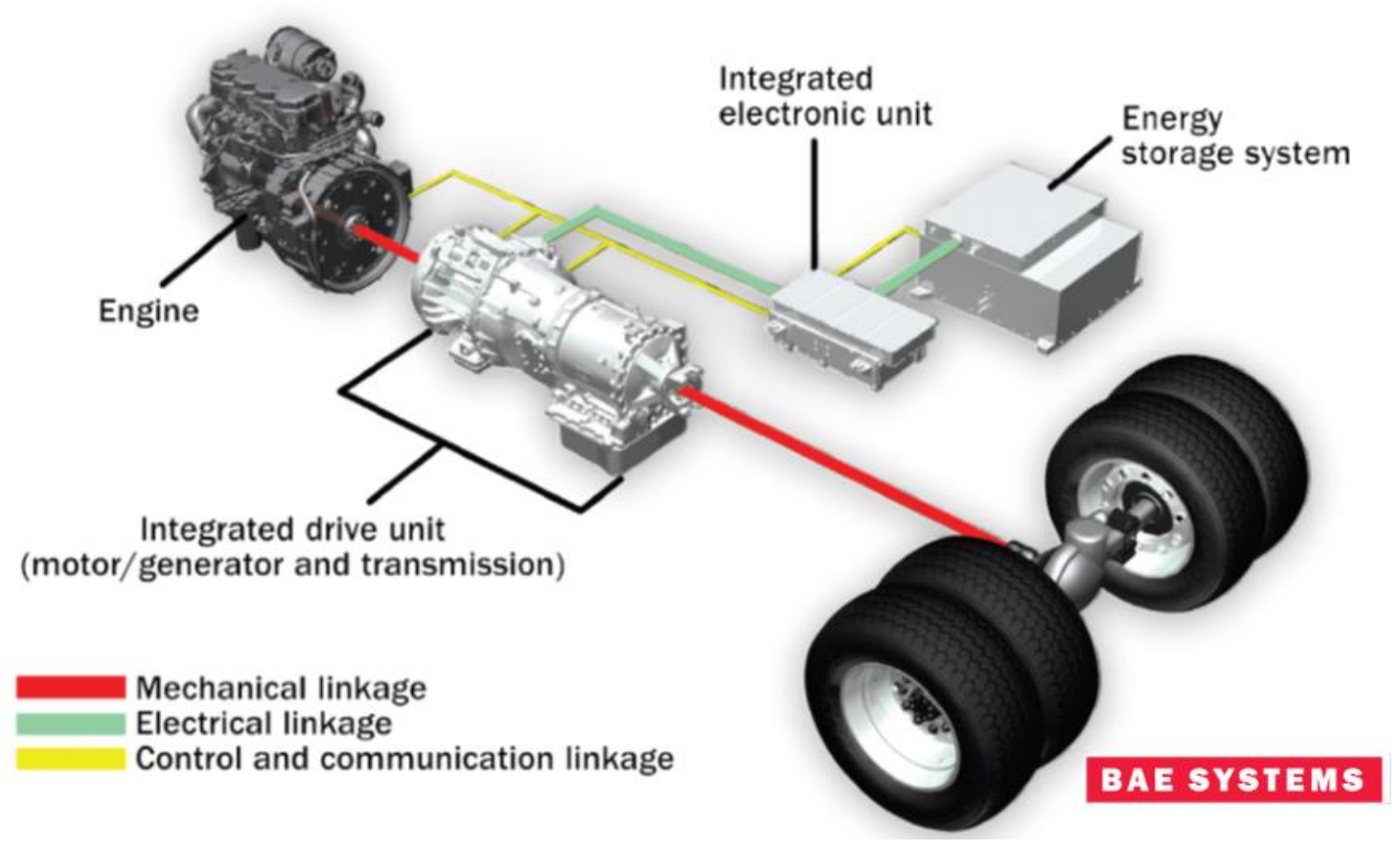

Figure 3.2: Schematic of pre-transmission hybrid drives [23] 


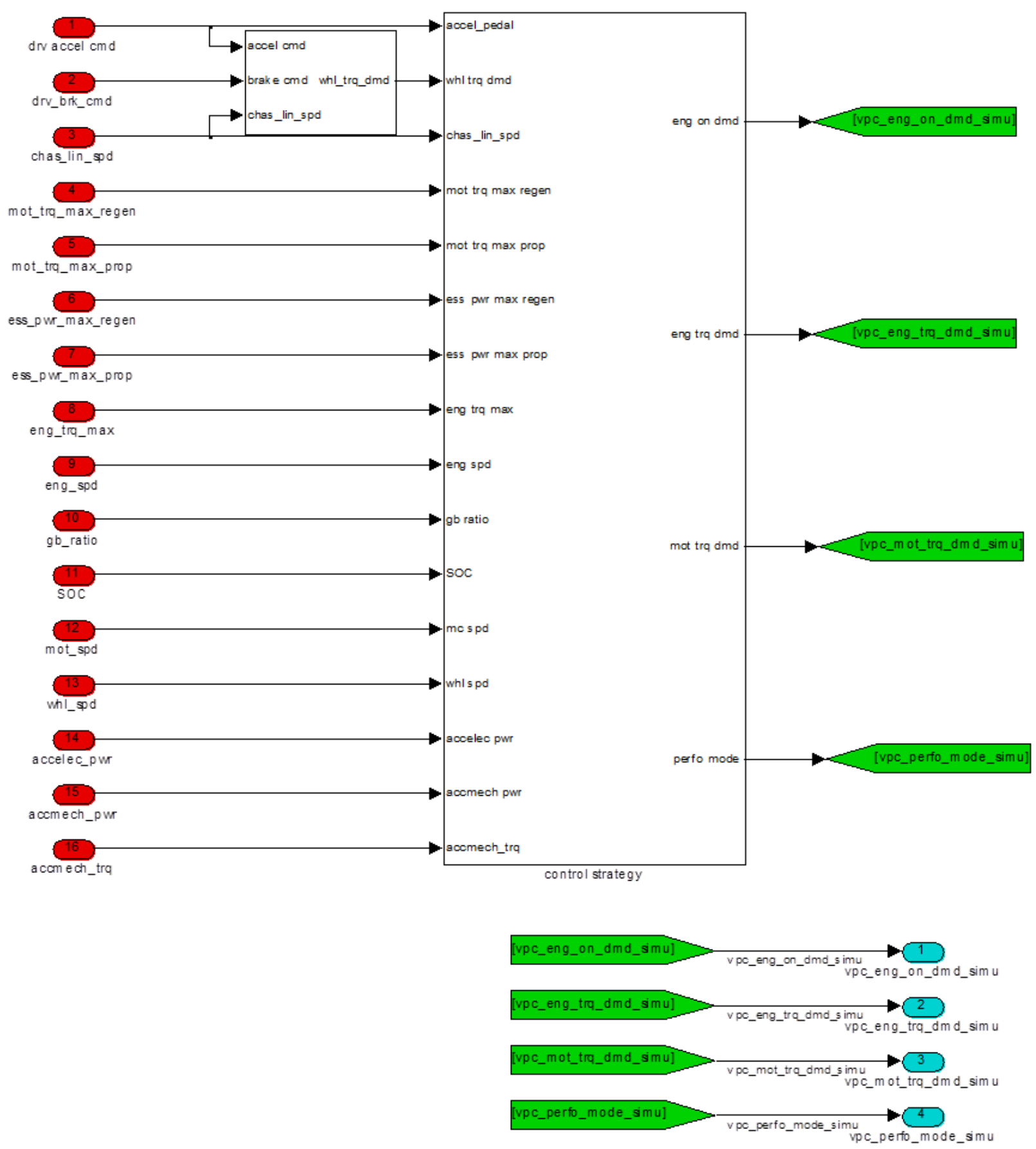

Figure 3.3: The control strategy for direct parallel pre-transmission HEV [54] 


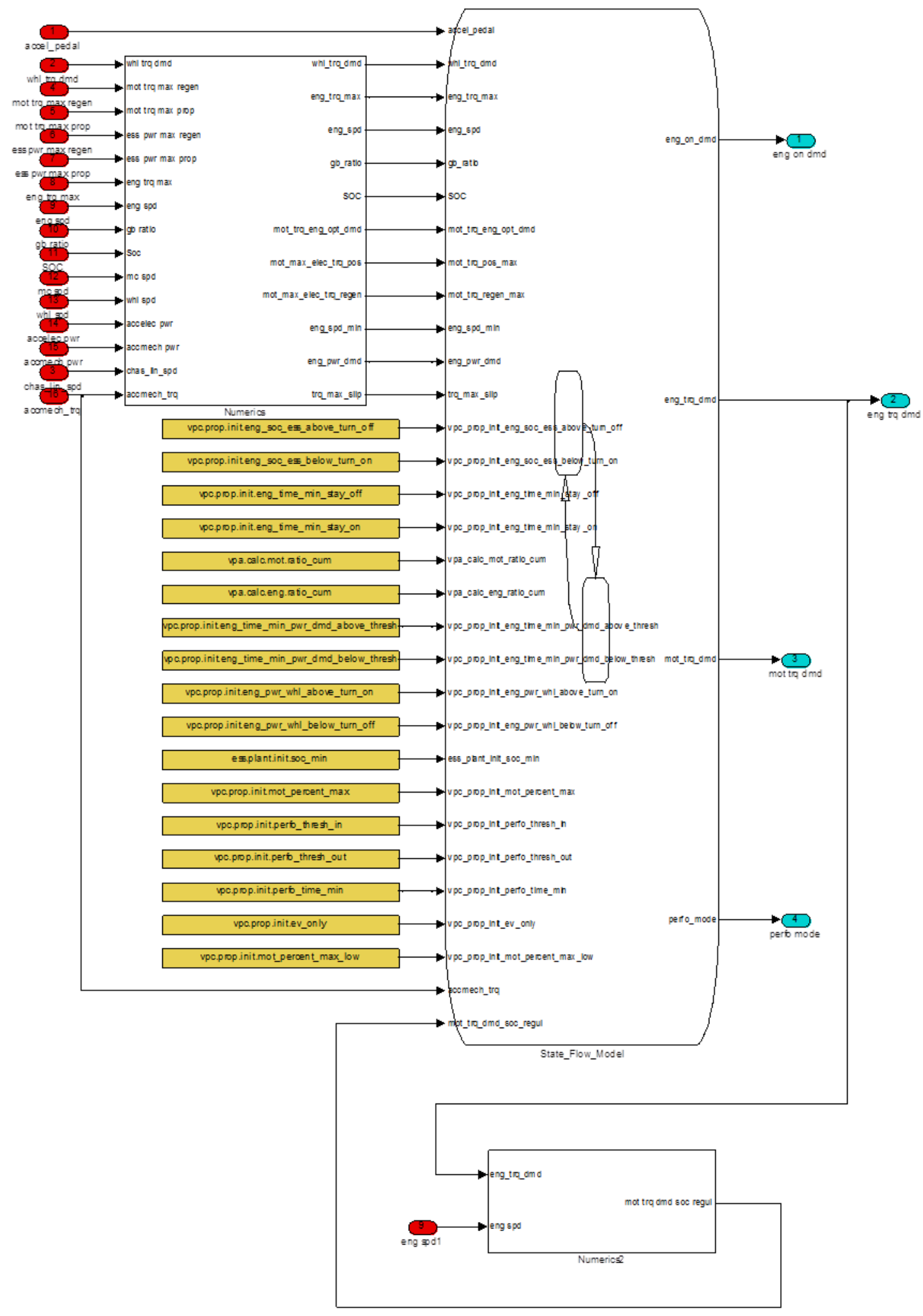

Figure 3.4: Diagram for subsystem control strategy for direct parallel pre-transmission HEV [54] 


\subsubsection{The vehicle propulsion controller design description}

The forward-looking simulation tool, Autonomie, was used in this study to simulate different vehicle models. It works by simulating the appropriate driver for the development of the accelerator and brake pedal inputs in order to meet the target vehicle speed. Developers can build a model car properly by selecting the engine, components, configuration files (the response time and equipment's efficiency maps) and control strategy and graphical user interface. As shown in

previous Figures (3.3 and 3.4) the VPC worked basically by gathering the driver demands, which is depressing the accelerator or the braking pedal inputs, and all other component characteristics. The components such as engine, transmission, electric motor and battery have maximum and minimum capability. Moreover, the controller considered several other parameters such as time for equipment to respond, efficiency map, mechanical and electrical power sources.

For example, when the driver depressed the accelerator pedal to move the vehicle, the VPC managed the powertrain's power to decide the power demand of each power source (i.e. engine and electric motor). This decision was based on the equipment energy and the vehicle activity. The equipment has initialization files that enable the designer to define different controlling parameters, such as engine on/off status, maximum power from the electric motor, and driving mode. Moreover, if the vehicle was being driven at low speed and the electric motor power was sufficient to propel the vehicle as well as enough energy in the ESS, then the VPC will make the vehicle run on electric mode as an electric vehicle (EV) to improve FE. On the other hand, when the driver hits brake pedal, the VPC will manage the powertrain to capture as much free energy as possible from using the regenerative brake system. This amount of captured free energy depends on the electric generator and ESS capacities. Figure (3.5) shows the state flow chart of the model and how it manages the power sources based on power events [54]. Furthermore, some braking is always supplied to the steering tires to maintain vehicle stability. 

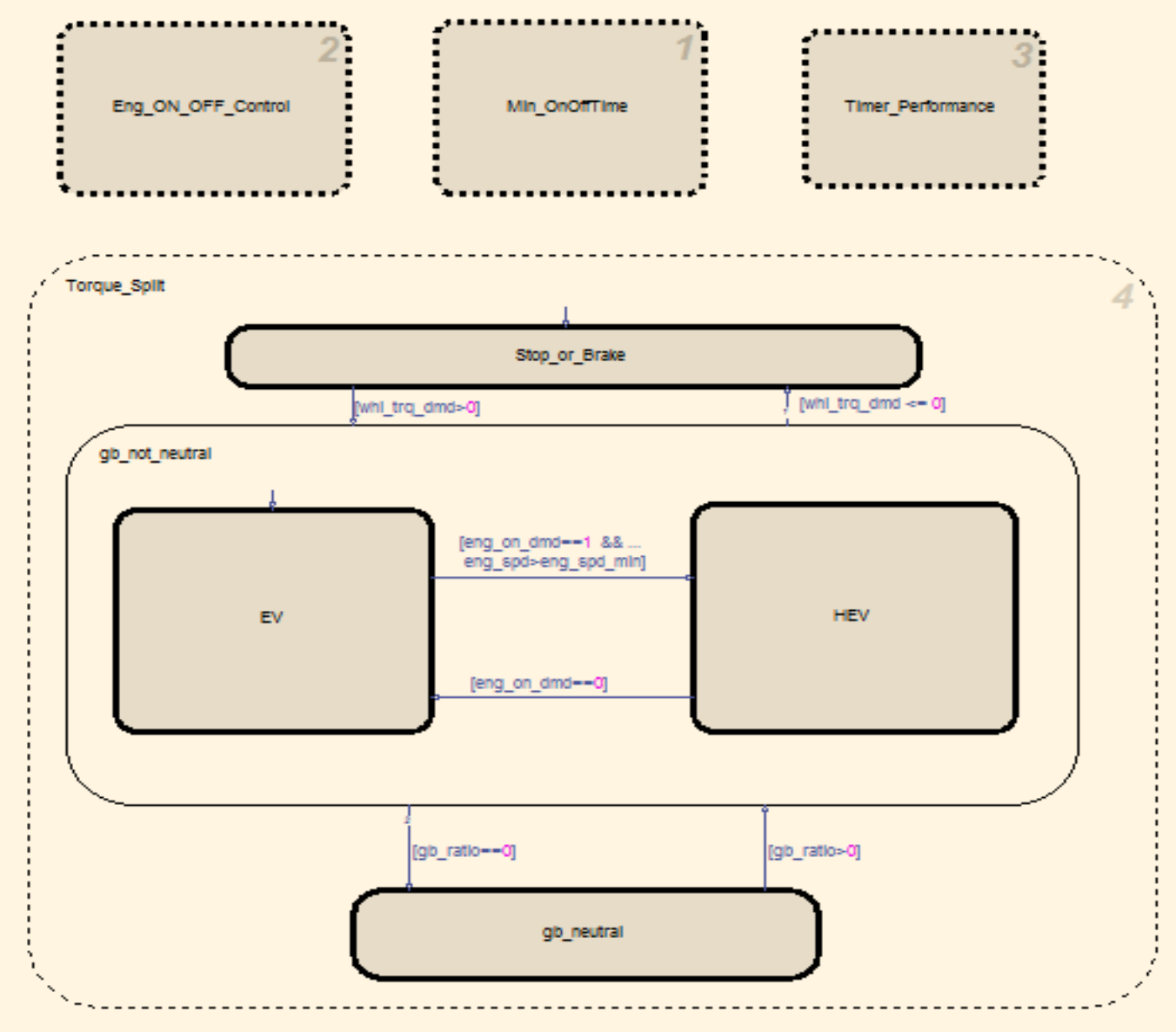

Figure 3.5: State flow (chart) for the VPC of parallel pre-transmission hybrid electric vehicle [54]

\subsubsection{Parallel HEV power sources management}

The sub charts of state flow show how the VPC manages the powertrain to propel the wheels or to capture the free energy while using the brake system. Figures (3.6-3.9) show in detail the coding and commands inside these sub charts. All of these commands together work to manage the parallel hybrid electric vehicle powertrain to propel the wheels. Based on the engine's power demand, the VPC managed the engine's on/off status. For example, when the power demand was less than $50 \mathrm{~kW}$, the engine will be off. There is a time period considered for the engine to respond the on command as shown in Figure (3.6). A hybrid electric vehicle can work as an electric vehicle depending on the alternative power source capability (i.e. electric motor and ESS) and the vehicle activity (i.e. mainly vehicle speed as well as acceleration and ascending hills). It was proved that the electric vehicle mode is more efficient in fuel economy perspective at low speed. On the other hand, it's proven that the vehicle is better to propel as a conventional vehicle (i.e. using engine only) at highway speed. Therefore, at high speed the alternative power 
source (i.e. the electric motor) would work as an assistant power source to assist the engine when the wheels' power demand surpasses the engine peak power, and it also works on capturing the free energy while using the brake system. The VPC could choose the power source to propel the wheels based on that strategy as shown in Figures (3.7 -3.8).

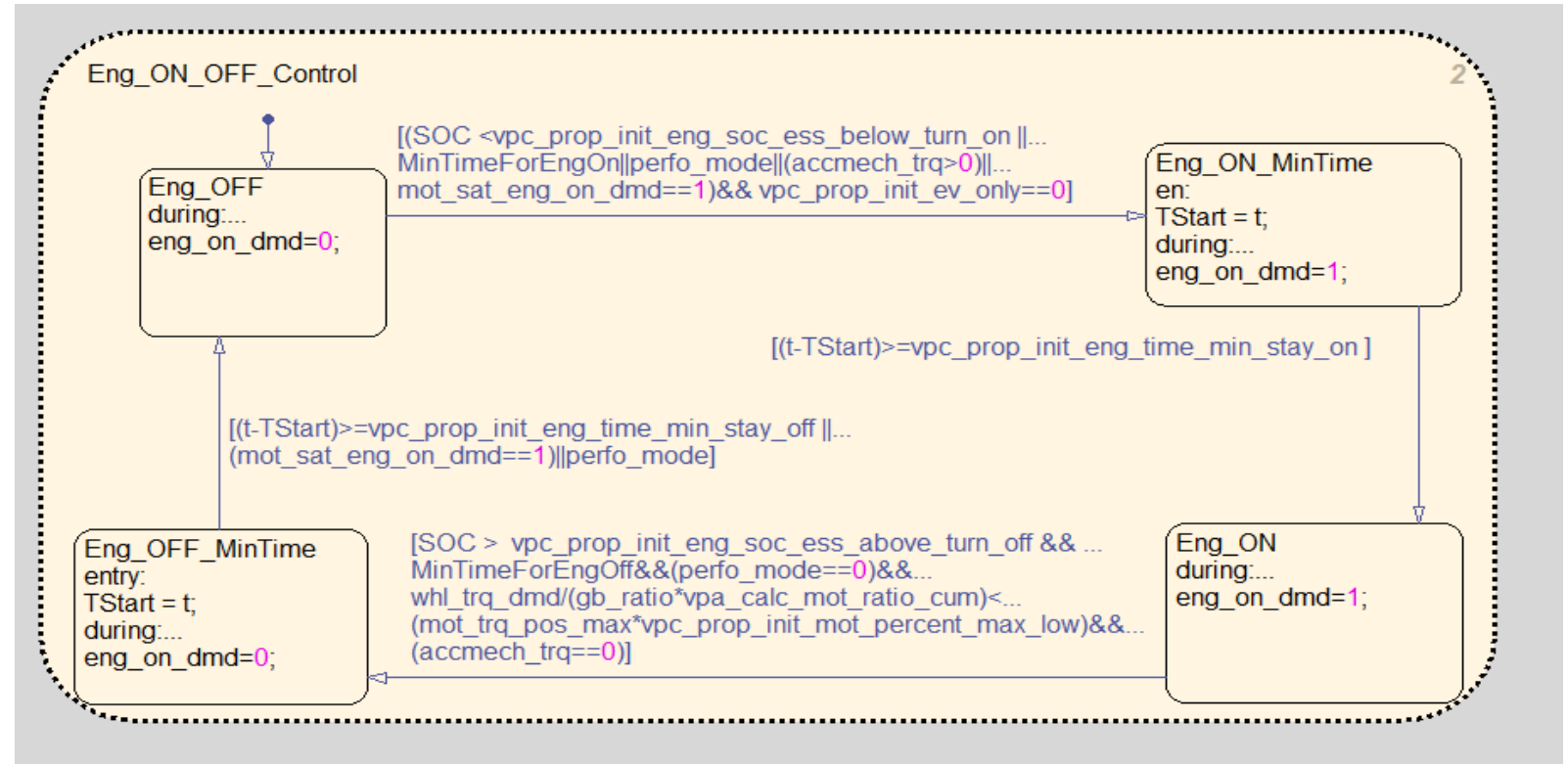

Figure 3.6: State flow (sub chart) for the VPC of parallel pre-transmission hybrid electric vehicle to control engine on/off status [54]

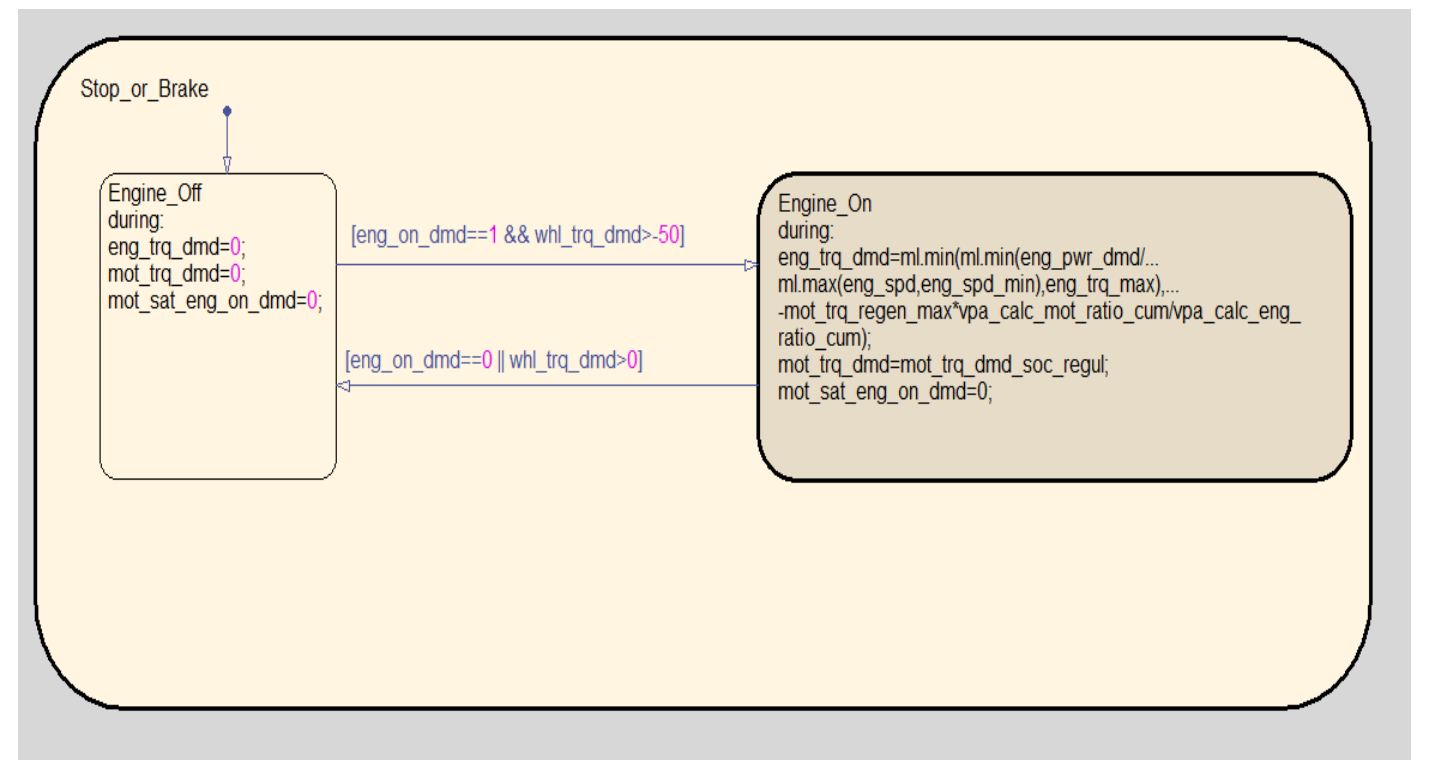

Figure 3.7: State flow (sub chart) for the VPC of parallel pre-transmission hybrid electric vehicle to manage the powertrain at stop or the brake status [54] 


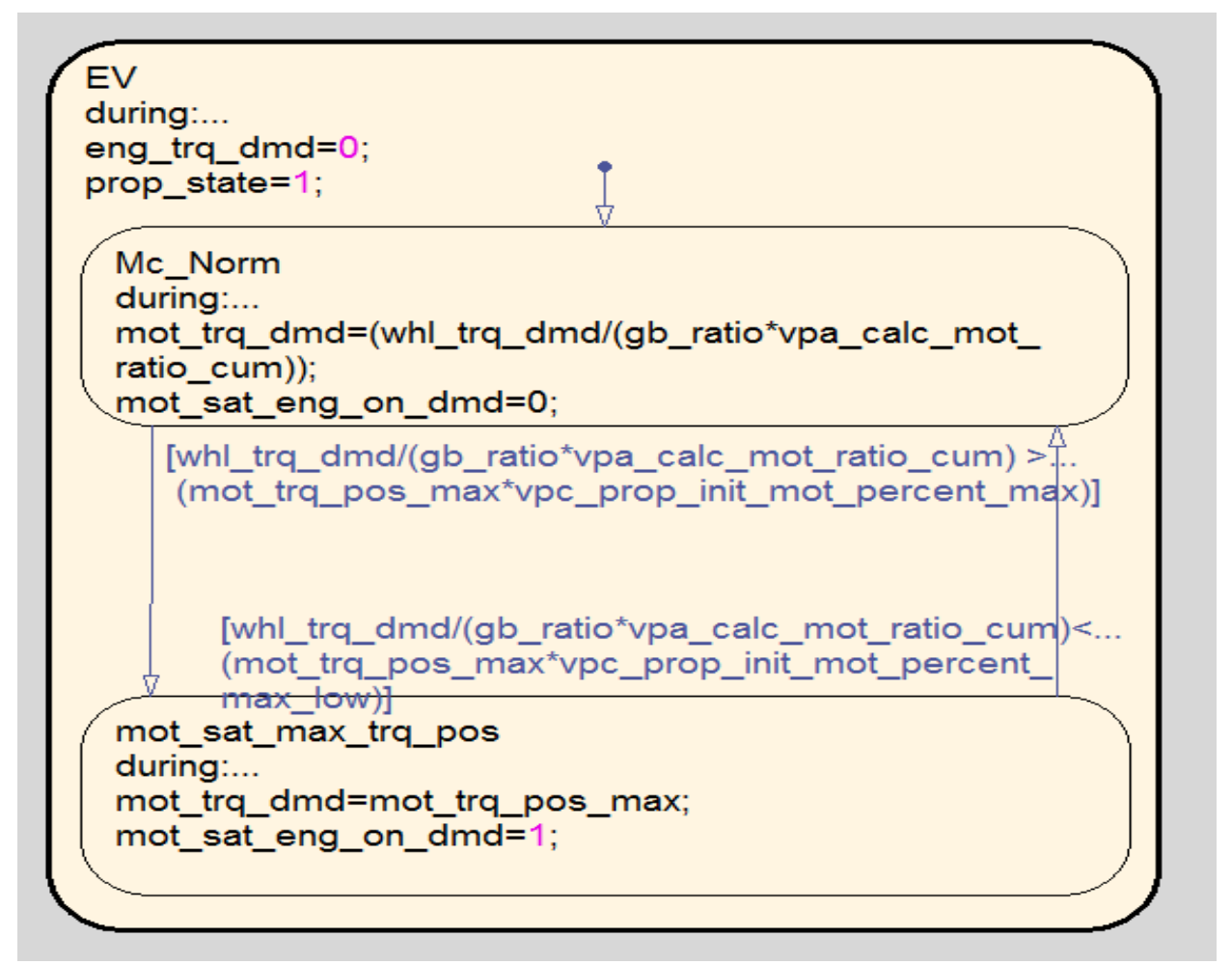

Figure 3.8: State flow (sub chart) for the VPC of parallel pre-transmission hybrid electric vehicle to run the vehicle on electric mode [54]

\subsection{Model and validate a heavy duty vehicle}

A conventional heavy duty vehicle was modeled using Autonomie software and the main equipment sizes are listed in Table (3.1). A hybrid electric heavy duty vehicle model was built using Autonomie software also. The parallel hybrid electric heavy duty vehicle configuration was chosen for this study. The hybridization depth varied from $100 \mathrm{~kW}$ motor size and $5 \mathrm{kWh}$ battery pack energy to $200 \mathrm{~kW}$ motor size and $15 \mathrm{kWh}$ battery pack. 
Table 3.1: Equipment sizes summary

\begin{tabular}{|l|c|c|c|c|}
\hline & Conventional & HEV-1 & HEV-2 & HEV-3 \\
\hline Engine Power (kW) & 410 & 410 & 410 & 410 \\
\hline GVWR 50\%load (kg) & 26,000 & 26,000 & 26,000 & 26,000 \\
\hline GVWR 100\%load (kg) & 36,000 & 36,000 & 36,000 & 36,000 \\
\hline Final Drive (ratio) & $3.55: 1$ & $3.55: 1$ & $3.55: 1$ & $3.55: 1$ \\
\hline Tires (m radius) & $305 / 70 / \mathrm{R} 22.5$ & $305 / 70 / \mathrm{R} 22.5$ & $305 / 70 / \mathrm{R} 22.5$ & $305 / 70 / \mathrm{R} 22.5$ \\
\hline Motor (kW) & -- & 100 & 150 & 200 \\
\hline Battery Energy (kWh) & -- & 5 & 10 & 15 \\
\hline Battery Power $(\mathrm{kW})$ & --- & 100 & 150 & 200 \\
\hline Transmission & 10 speed $(14.8-1)$ & $10 \mathrm{speed}$ & 10 speed & 10 speed \\
& & $(14.8-1)$ & $(14.8-1)$ & $(14.8-1)$ \\
\hline Mechanical Acc. $(\mathrm{kW})$ & 5.2 & 1 & 1 & 1 \\
\hline Elec. Acc. $(\mathrm{kW})$ & 0.3 & 3 & 3 & 3 \\
\hline
\end{tabular}

\subsubsection{The tested conventional truck information}

The heavy duty vehicle used in this study was a Peterbilt truck, which is a conventional tractor, with a non-EGR 550 HP (410 kW) 1996 Caterpillar 3406E engine, 18-speed Roadranger manual transmission and Peterbilt 379EXHD tandem axle drive. A test was performed at the WVU Transportable Heavy Duty Vehicle Emission Testing Laboratory in April 2006. Acknowledgement is given to the engineers, staff, faculty, and graduate students that were accompanying with the testing operations within the Center for Alternative Fuels, Engines, and Emissions (CAFEE) at WVU. The vehicle was operated over the UDDS on the chassis dynamometer to provide continuous emissions as well as engine speed and engine percent load data [49]. Detailed information about the vehicle can be found in Table 3.2 [55]. 
Table 3.2: Details of the Peterbilt truck information [55]

\begin{tabular}{|c|c|}
\hline WVU Test Reference Number & WVU-Peterbilt-D2-TEST_D \\
\hline Vehicle Type & Tractor \\
\hline Vehicle Manufacturer & Peterbilt \\
\hline Vehicle Model Year & 1996 \\
\hline Vehicle tested weight (lb.) & 56,000 \\
\hline Odometer Reading (mile) & Started from 441,097 \\
\hline Transmission Type & 18-speed Manual Transmission \\
\hline Final drive ratio & 3.55 \\
\hline Tire Size & $305 / 70 \mathrm{R} 22.5$ \\
\hline Coefficient of Rolling Resistance & 0.00938 \\
\hline Coefficient of Aerodynamic & 0.79 \\
\hline Engine Type & Caterpillar 3406E \\
\hline Engine Model year & 1996 \\
\hline Primary Fuel & D2 \\
\hline Test Cycle & UDDS (also termed TEST_D) \\
\hline Test Date & $4 / 21 / 06$ \\
\hline
\end{tabular}

\subsubsection{Engine model}

The engine used in this study was a Caterpillar C15 with rated power of $324 \mathrm{~kW}$. The engine model size was scaled to match the tested engine power size $(410 \mathrm{~kW})$. The engine model in Autonomie considered engine torque demand, engine speed, and engine $\mathrm{ON}$ as input parameters, and it considered the fuel consumption rate as output. Figure (3.9) shows the general Simulink diagram of the engine model. 


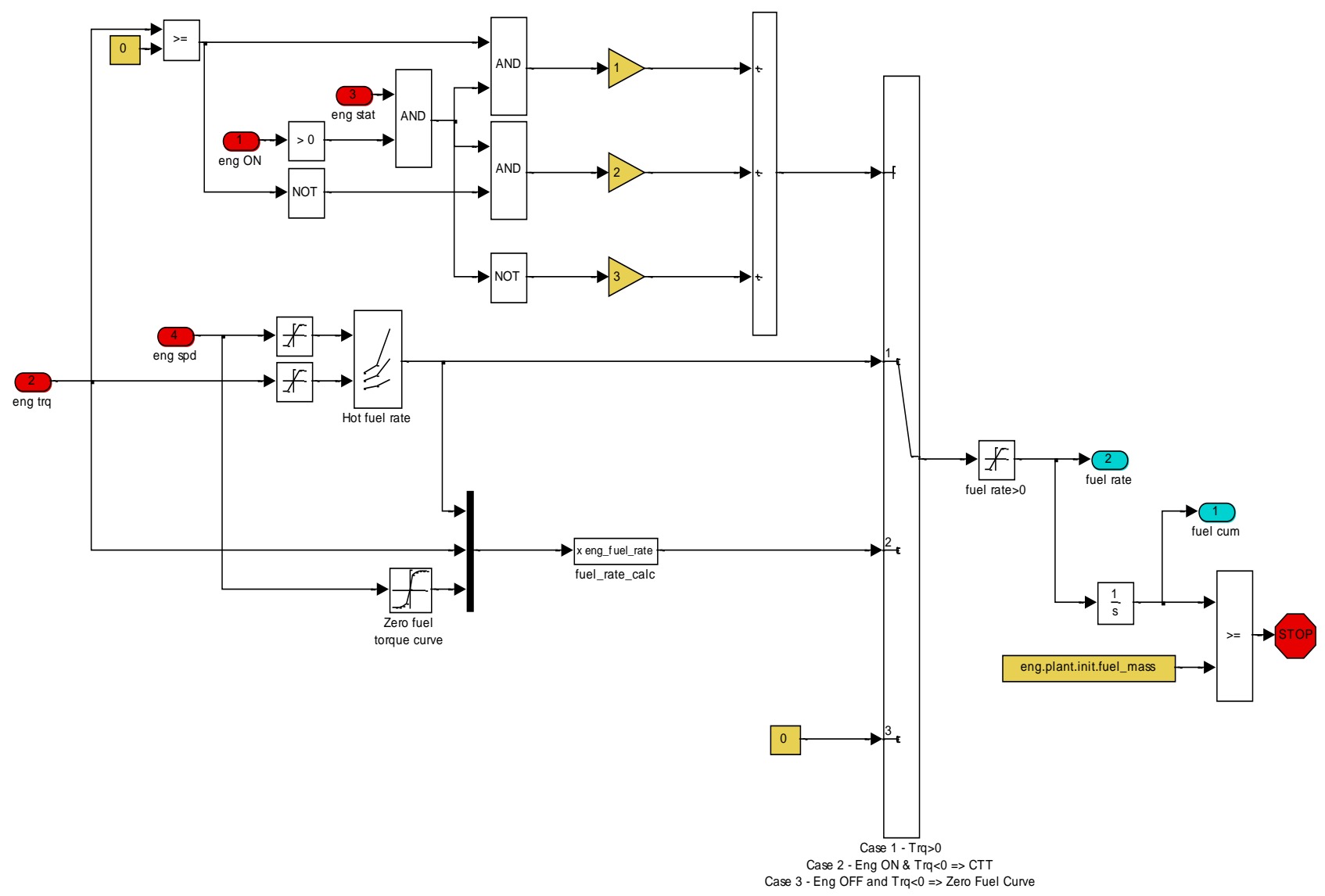

Figure 3.9: General Simulink diagram of the engine model in Autonomie [54]

\subsubsection{Validate simulation results}

When any model is simulated, a very important question must be answered. The question is, are these results for that model valid? An experimental measurement was used to validate the conventional vehicle model. The data used were for the truck owned by WVU/CAFEE as presented in previous section. The truck $(410 \mathrm{~kW})$ was run through the UDDS on the chassis dynamometer to provide fuel rate, engine speed and engine percent load. Table (3.3) shows the measured [57] and simulated results respectively. Figures (3.10-3.14) show the measured results verses the simulated test for the conventional truck model on the UDDS driving cycle. In this 
study, a parallel hybrid electric vehicle wasn't tested, but the model of it has been simulated using Autonomie. Moreover, the same validated conventional truck model was used after adding the hybridization equipment, which was electric motor, battery pack, and vehicle propulsion controller. Therefore, the outcomes of this study can be considered as reliable results.

Table 3.3: Validation results of peterbilt truck

\begin{tabular}{|l|c|c|c|}
\hline Parameter & Measured & Autonomie Simulated & Relative \% Error \\
\hline UDDS cycle (mile) & 5.44 & 5.4 & 0.74 \\
\hline Average vehicle speed (m/s) & 8.35 & 8.34 & 0.03 \\
\hline Fuel economy (MPG) & 3.82 & 3.65 & 4.4 \\
\hline Fuel mass (kg) & 4.58 & 4.71 & -2.84 \\
\hline Average Engine fuel rate (g/s) & 4.37 & 4.49 & -2.79 \\
\hline Average Engine speed $(\mathrm{rad} / \mathrm{s})$ & 124.06 & 107.76 & 13 \\
\hline Average Engine torque $(\mathrm{Nm})$ & 498.93 & 510.10 & -2.24 \\
\hline Average Engine power $(\mathrm{kW})$ & 79.59 & 70.82 & 11 \\
\hline \hline Average $\mathrm{CO}_{2}(\mathrm{~g} / \mathrm{mile})$ & 2640.49 & 2764.36 & -4.7 \\
\hline
\end{tabular}

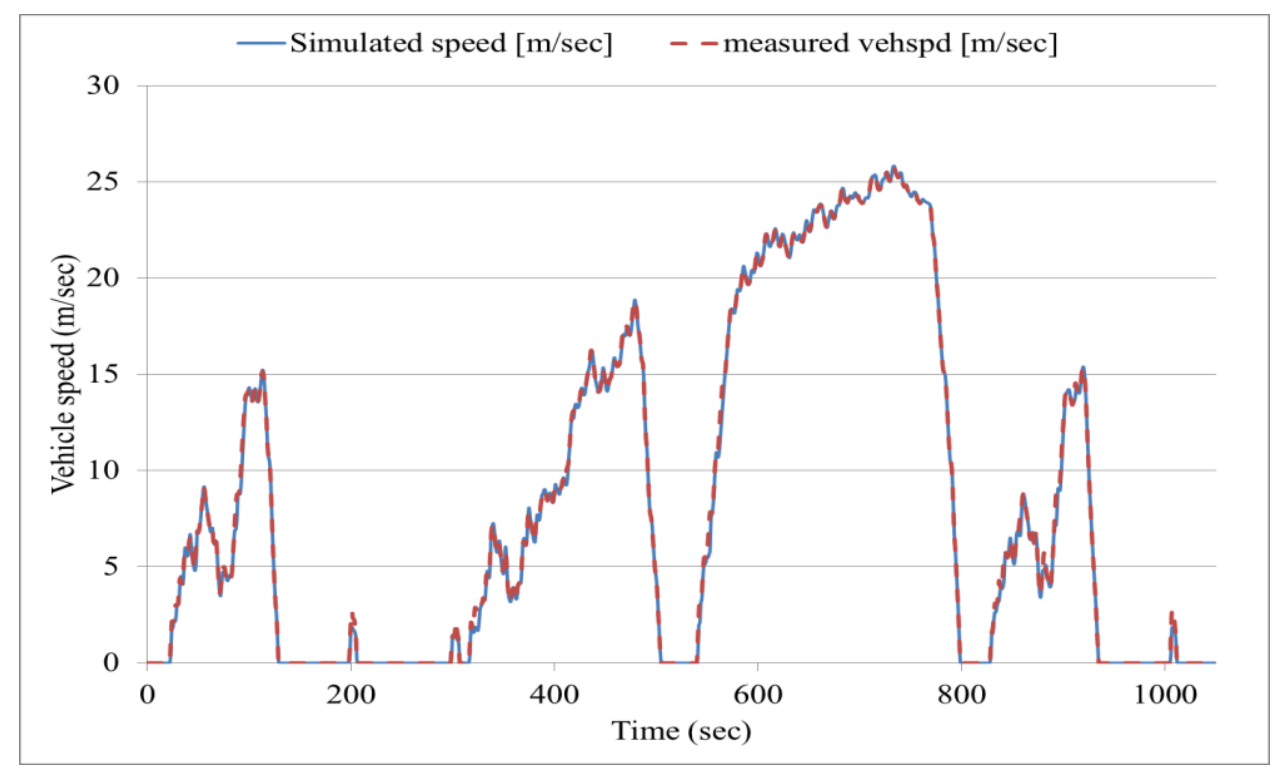

Figure 3.10: Measured versus simulated vehicle speed. 


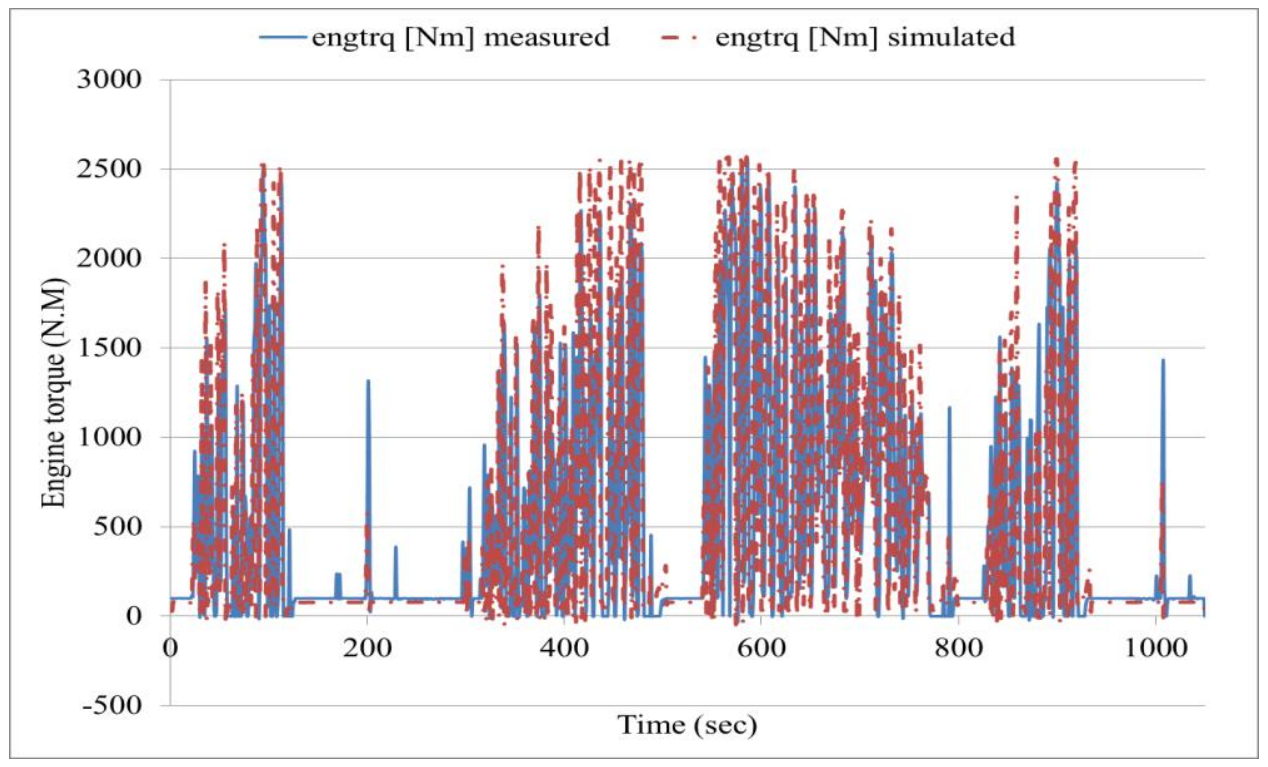

Figure 3.11: Measured versus simulated engine torque.

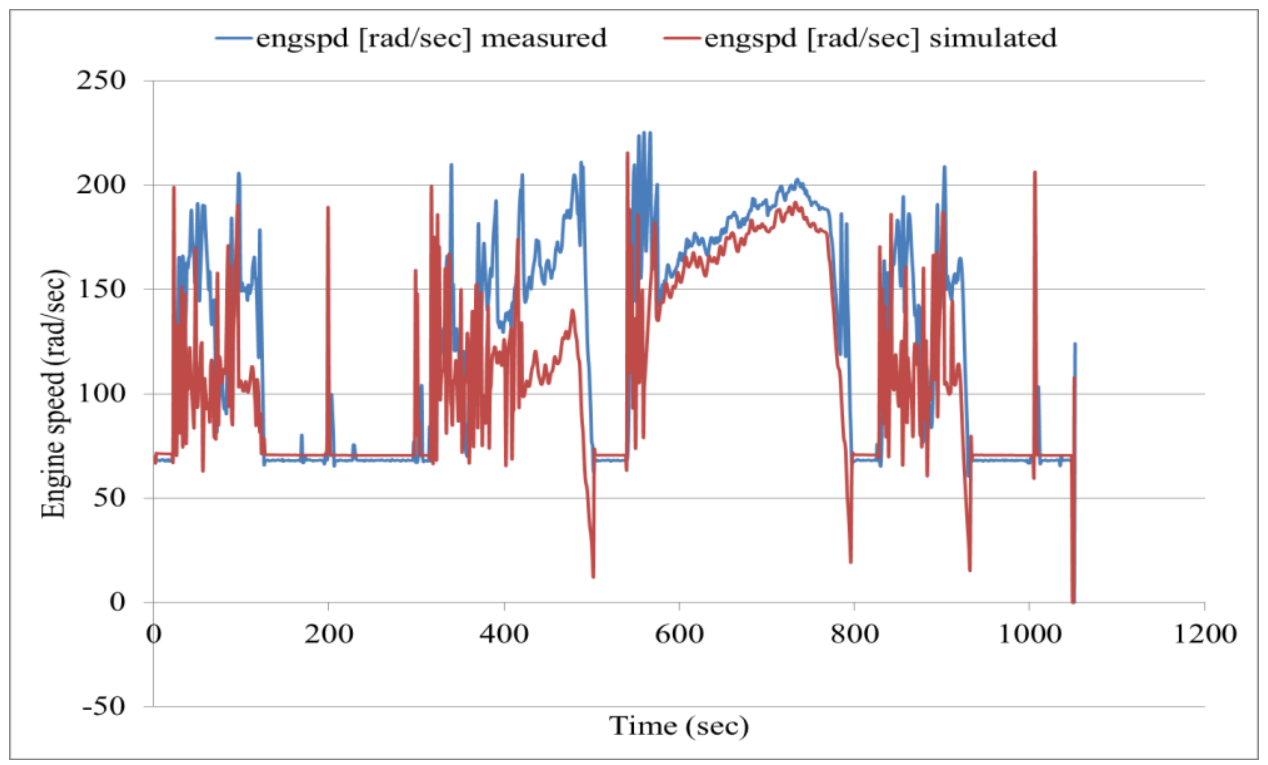

Figure 3.12: Measured versus simulated engine speed. 


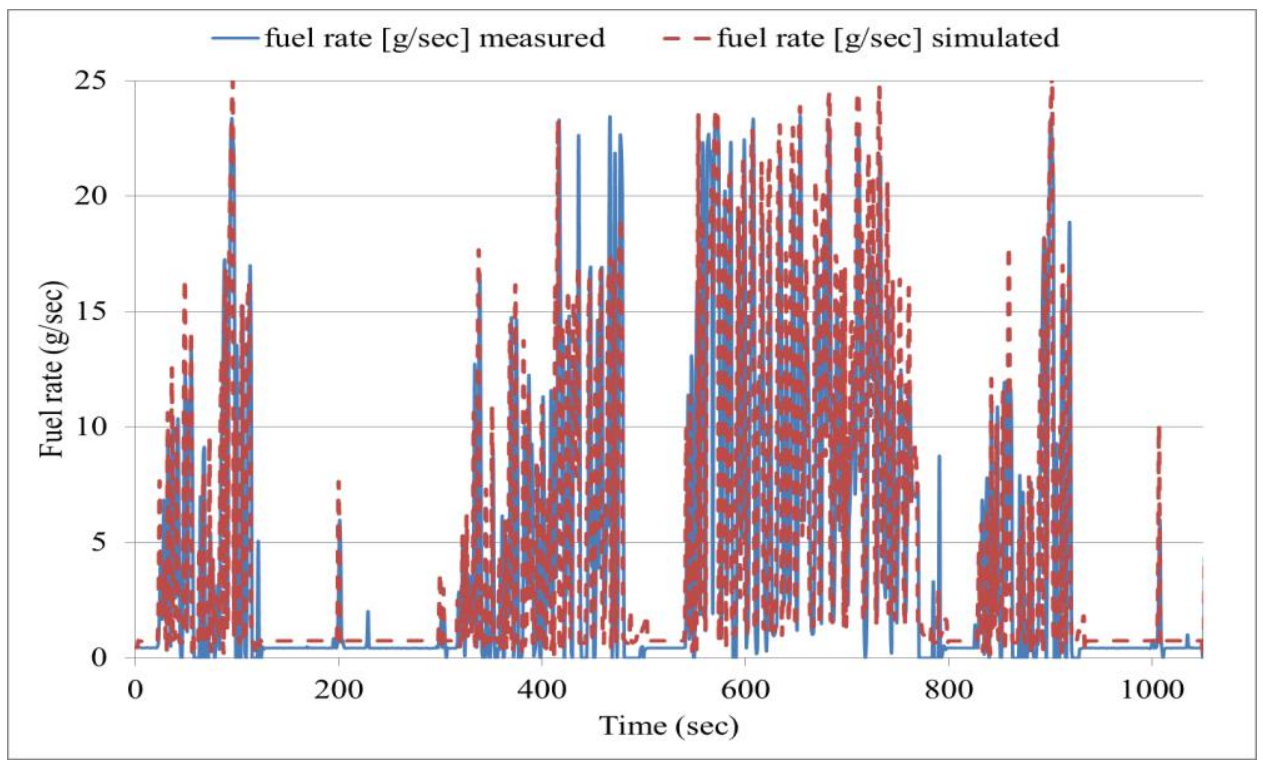

Figure 3.13: Measured versus simulated fuel rate.

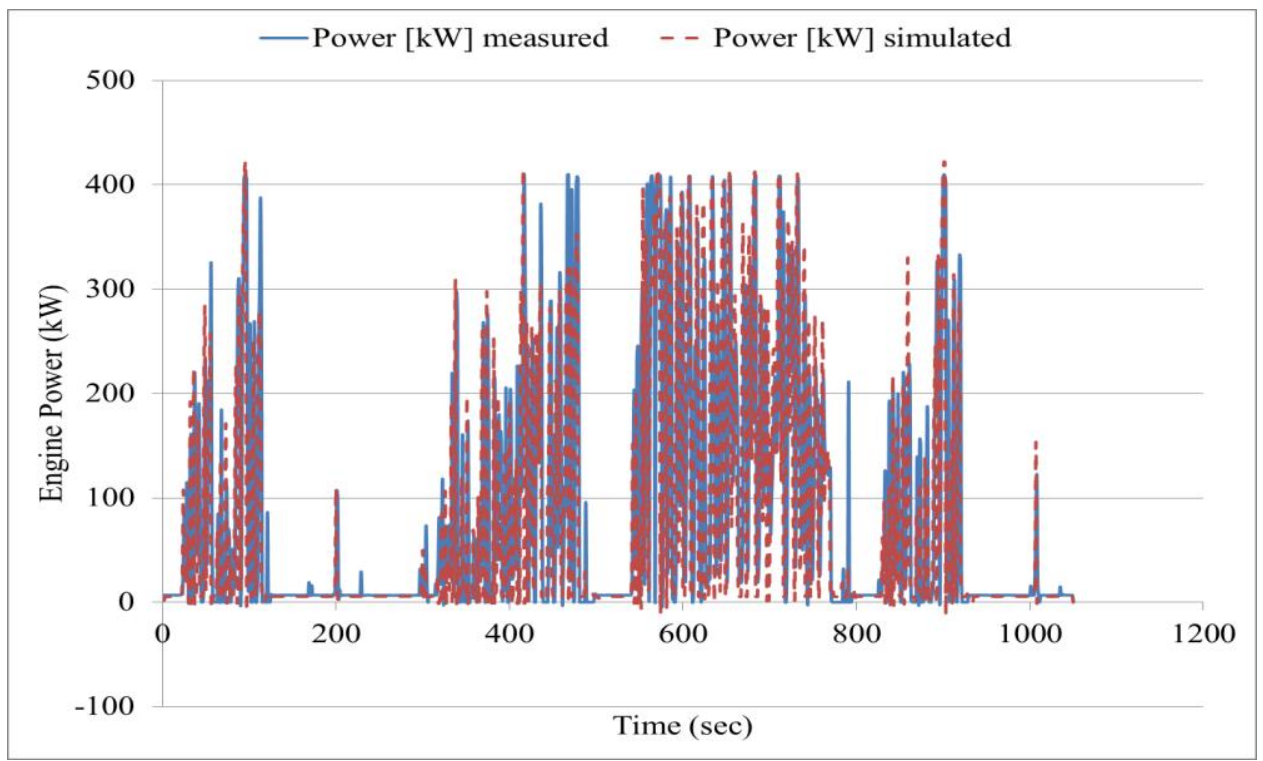

Figure 3.14: Measured versus simulated output engine power. 


\subsection{Several uphill and downhill terrains with highway constant speed cycles}

The ultimate goal of modifying the VPC was to enable it to manage the ESS in opportunistic regenerative braking. On the other hand, the looking-ahead control should be precise in estimating the upcoming power demand. Therefore, ascending and descending hill with constant speed has been selected to analyze the modified model sensitivity. Moreover, a constant highway speed driving cycle (60 mile/hour) has been selected. Road grade was generated as 3\% for uphill and downhill terrain as shown in Figures (3.15 -3.17). Figure (3.18) shows the different road grade as going up and down hill which is $0.5 \%, 2 \%$, and $3 \%$, respectively. The purpose of generating different road grades was to investigate the modified VPC model detection effectiveness. Since, the road grade between $1 \%$ and $-2 \%$ does not need the driver to depress the gas pedal or hit the brake to maintain vehicle speed as proven in previous study [35], so the road grade for terrains have been selected in between and out of the values. In other words, when the truck ascends a road with less than $1 \%$ grade, the powertrain does not need substantial additional power to maintain the truck speed.

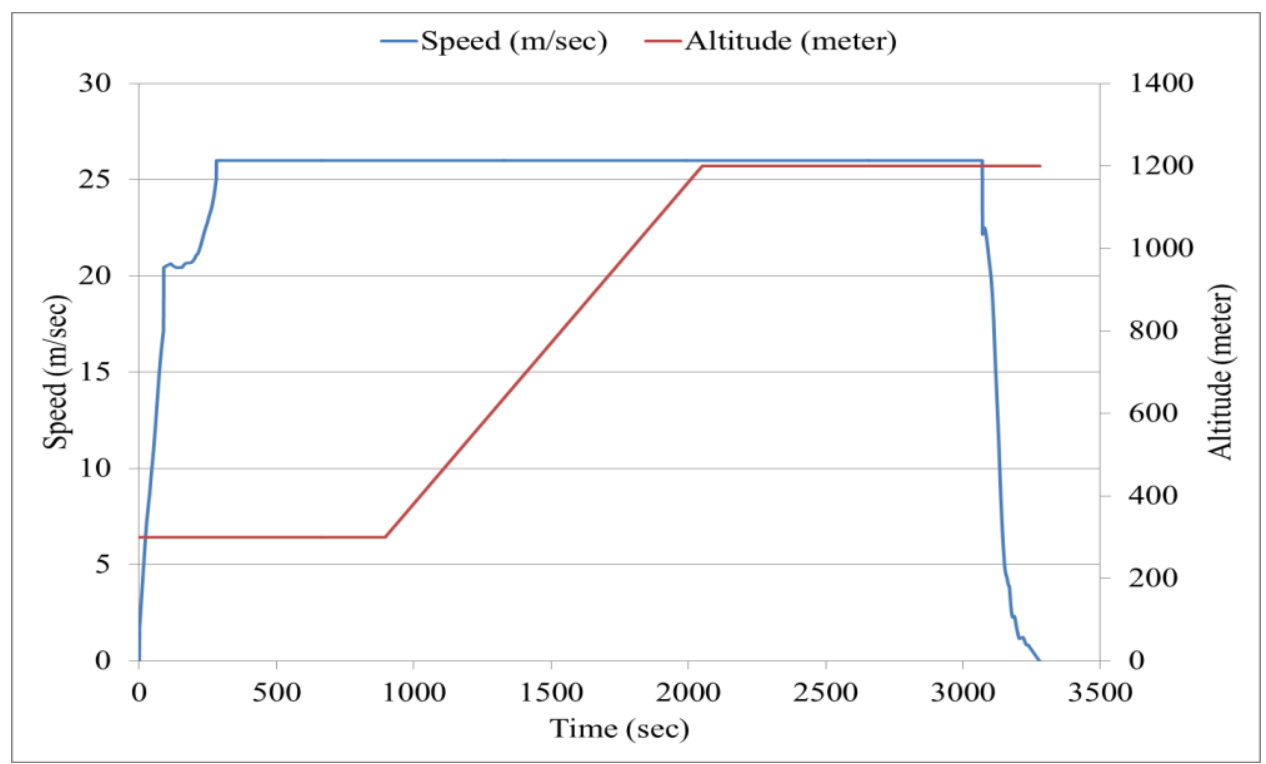

Figure 3.15: Ascending hill with 3\% road grade 


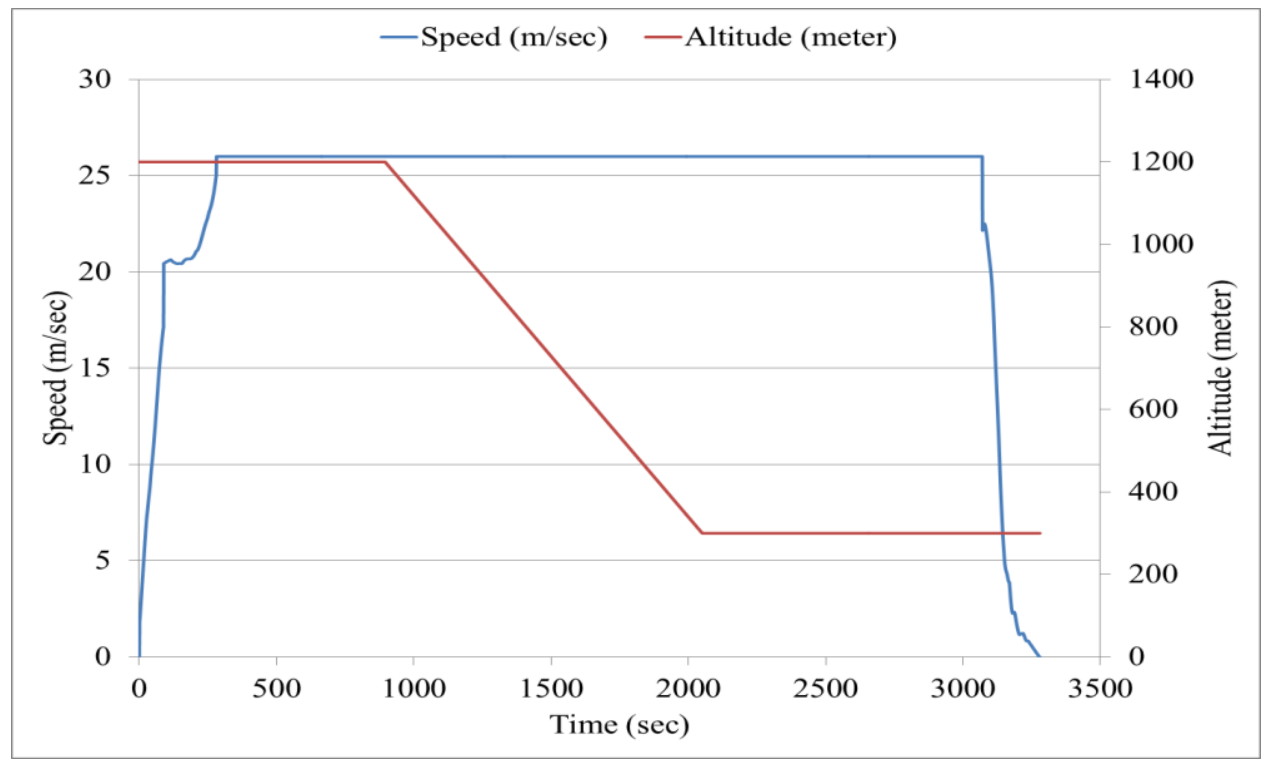

Figure 3.16: Descending hill with 3\% road grade

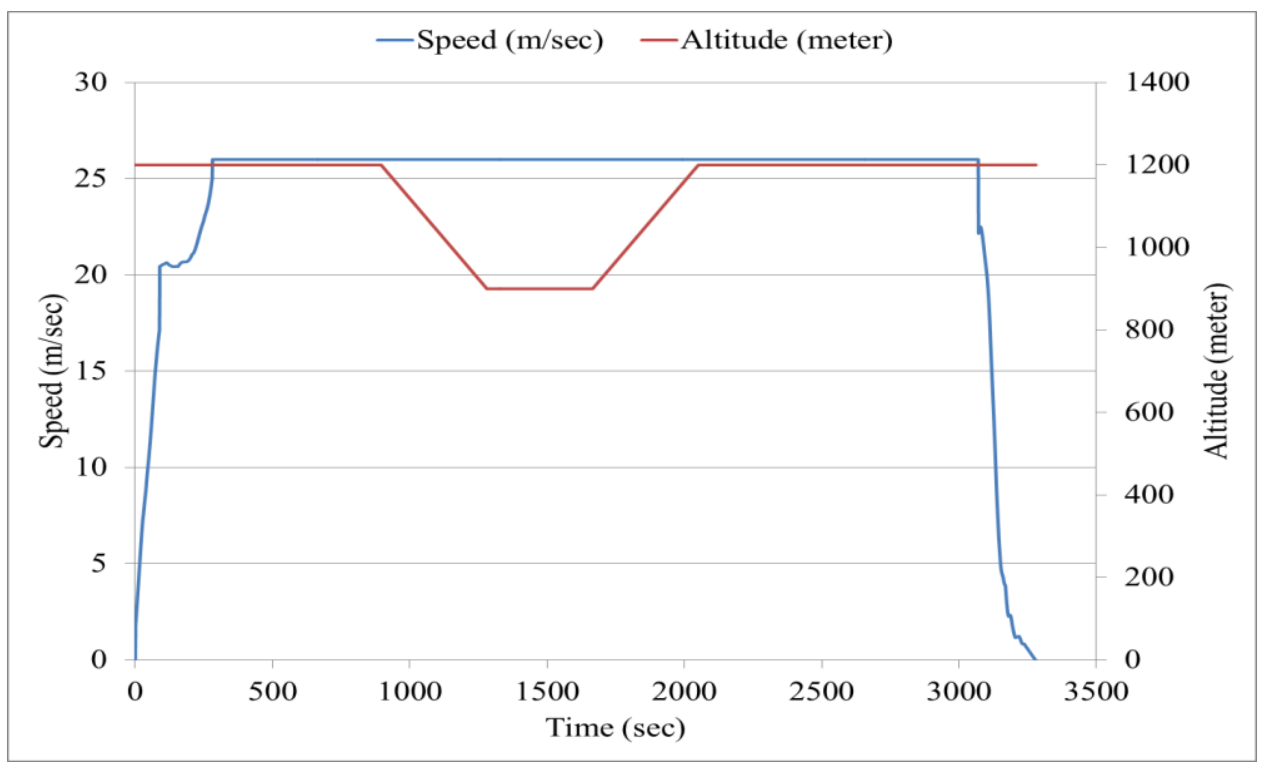

Figure 3.17: Descending and Ascending hill with 3\% road grade 


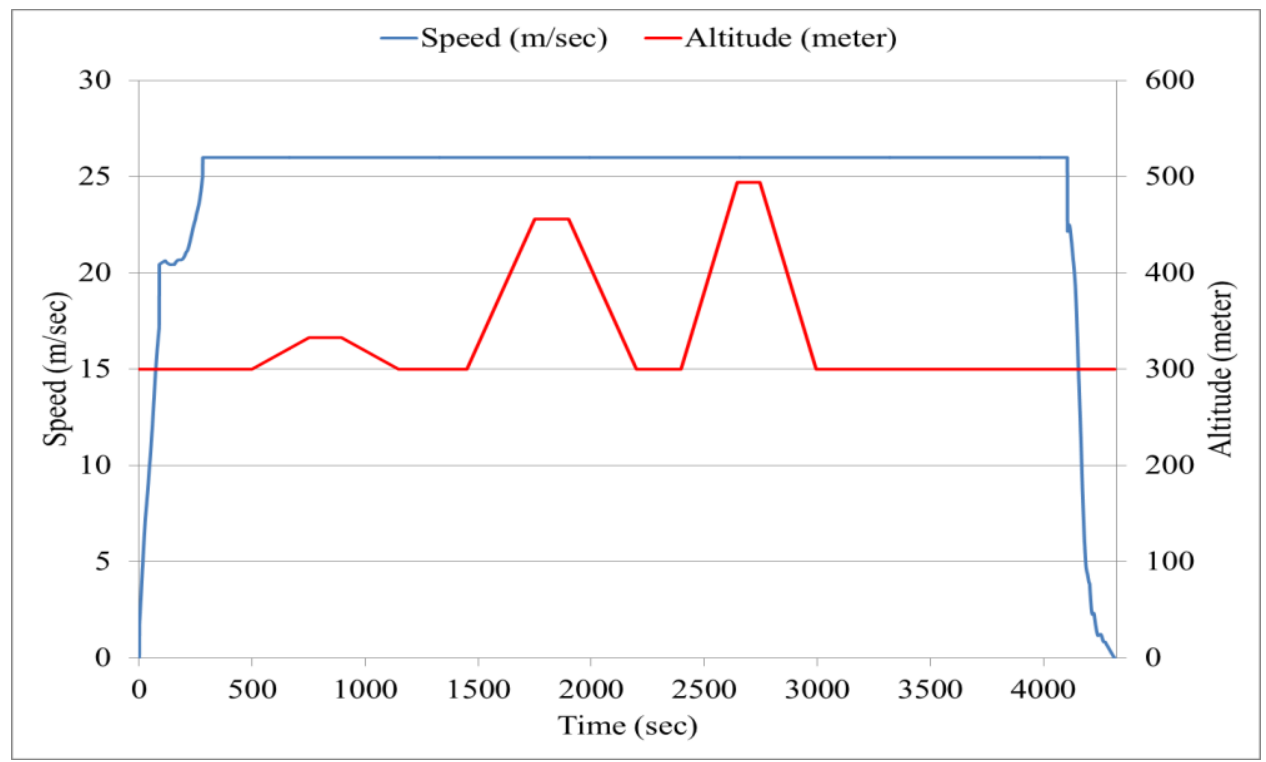

Figure 3.18: Ascending and Descending hill with $0.5 \%, 2 \%$ and $3 \%$ road grades respectively

\subsection{Identify different real world driving cycles for heavy duty vehicles}

This section identifies the real world data source and summarizes some important characteristics for these cycles. The data source, which were used in this study, is representative of in-use measurements achieved with PEMS and WVU MEMS units [56]. Appreciation is given to the engineers, staff, faculty, and graduate students that were associated with the testing campaigns within the CAFEE at WVU. Vehicles used to collect this data were heavy duty vehicles with GVWR of 80,000 lbs. The MEMS consisted of an exhaust gas sample conditioning system and emissions gas analyzers for $\mathrm{NO}_{\mathrm{X}}$ and $\mathrm{CO}_{2}$ concentrations, a data acquisition system, an exhaust flow rate measurement device, ECU interface hardware, ambient condition sensors and a global positioning system (GPS) receiver. For this study, ECU, ambient condition sensors, barometer, and GPS were the main sources for vehicle speed and road grade data. 


\section{Routes used}

Five routes were used in this study. These routes represent different highway patterns with different road grades [57].

\section{West Virginia University to California route}

The accumulative time for this trip was about 42 hours driving and about 2,500 miles in distance. Three sections were selected during this trip and they are as follows:

Columbus, $\mathrm{OH}$ to Indianapolis, IN (Clmbs2Ind) route: This route represented a highway and flat terrain journey on I-70 for about 170 miles.

Las Vegas, NV to Halloran Summit Road, Nipton, CA (Las2Hall) route: This route represented uphill driving on highway I-15 for about 70 miles.

Halloran Summit Road, Nipton, CA to Barstow, CA (Hall2Bar) route: This route represented downhill driving on highway I-15 for about 80 miles.

\section{Bruceton Mills route:}

This route was separated into outbound (Sab2BM) and homecoming (BM2Sab) trips. These two routes have a significant hill climb during each route and provided more than 10 minutes of continuously high power demand data. The route began at the West Virginia University Sabraton facility near to the entrance ramp on I-68 east and nonstop on I-68, where a climb of a constant $5 \%$ grade existed, followed by transient road grades to the reversal point at Bruceton Mills, WV. The total trip distance was about 40 miles. The highway speed limit was $70 \mathrm{mph}$ on the going trip; however, the highway speed limit was $50 \mathrm{mph}$ on the way back to the WVU facility in Sabraton when the route was steeply descending. Table 3.4 gives additional information and characteristics of the real world routes.

Figures (3.19-3.23) show the real world driving cycles used in this study. The $1 \%$ and $-2 \%$ road grade have been selected because the driver between these values does not need to depress the gas pedal or depress brake to maintain vehicle speed as shown in previous study [35]. In other words, when the truck ascends a road with less than $1 \%$ grade, the powertrain will not need a substantial additional power to maintain the truck speed. 
Table 3.4: Characteristics of real world condition cycles.

\begin{tabular}{|l|l|c|c|c|c|c|c|c|}
\hline & & Ave Speed & $\begin{array}{c}\text { Max } \\
\text { Speed }\end{array}$ & $\begin{array}{c}\text { Grade } \\
\mathbf{>} \mathbf{1 \%}\end{array}$ & $\begin{array}{c}\text { Grade } \\
\mathbf{< - 2} \%\end{array}$ & Distance & Duration & $\begin{array}{c}\text { Time } \\
\text { stop }\end{array}$ \\
\hline & & $\mathrm{mph}$ & $\mathrm{mph}$ & $\%$ & $\%$ & mile & hour & $\%$ \\
\hline $\mathbf{1}$ & Clmbs2Ind & 61.21 & 70.17 & 6.80 & 1.17 & 170.61 & 2.78 & 0.25 \\
\hline $\mathbf{2}$ & Las2Hall & 57.94 & 70.53 & 25.17 & 5.54 & 81.69 & 1.41 & 0.49 \\
\hline $\mathbf{3}$ & Hall2Bar & 53.96 & 65.9 & 20.20 & 27.82 & 72.58 & 1.34 & 0.53 \\
\hline $\mathbf{4}$ & BM2Sab & 31.08 & 70.32 & 30.9 & 35.66 & 20.29 & 0.65 & 10 \\
\hline $\mathbf{5}$ & Sab2BM & 33.02 & 71.71 & 42.52 & 25.20 & 20.66 & 0.62 & 15.75 \\
\hline
\end{tabular}

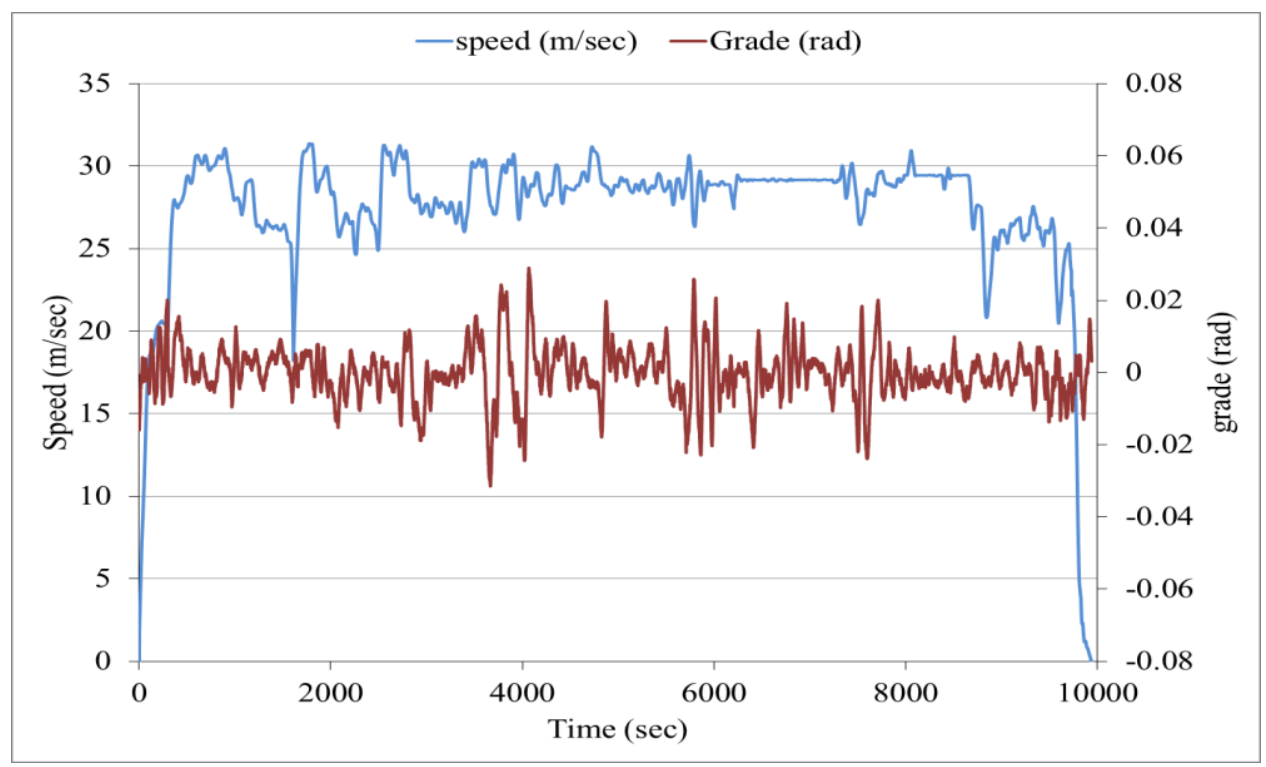

Figure 3.19: Columbus, $\mathrm{OH}$ to Indianapolis, IN real world driving cycle [56] 


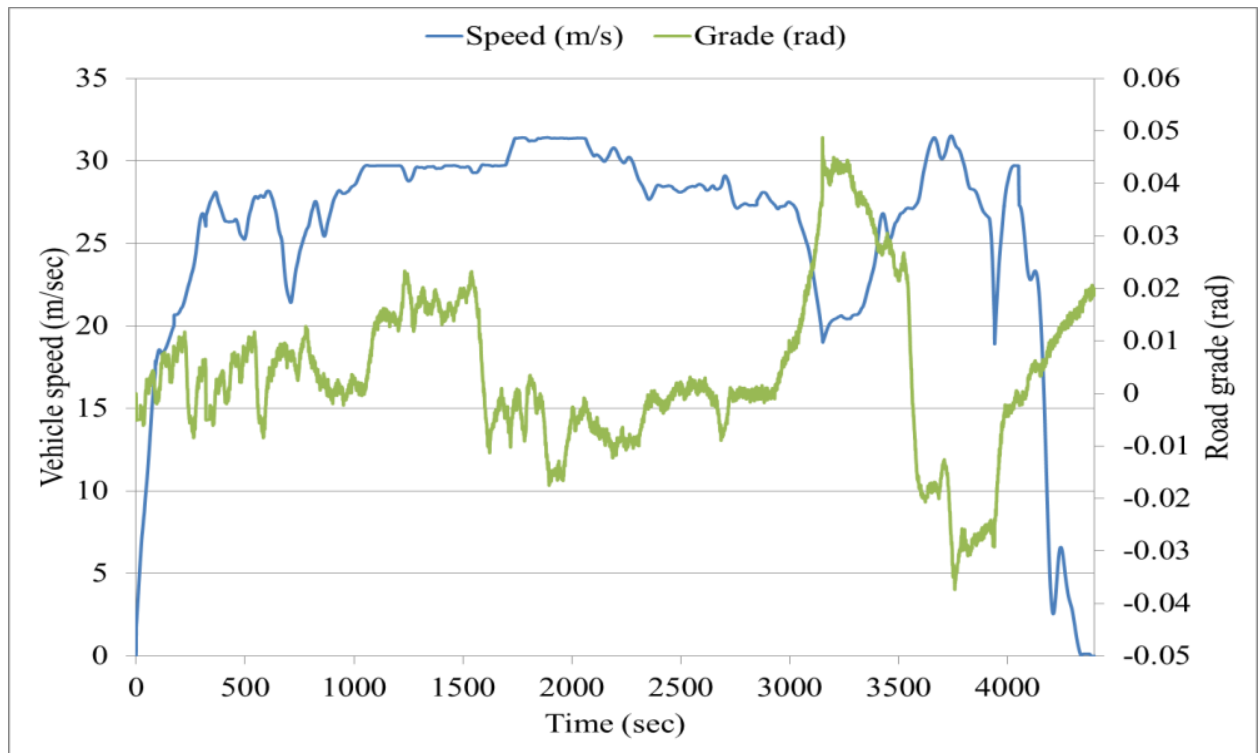

Figure 3.20: Las Vegas, NV to Halloran Summit Road, Nipton, CA real world cycle [56]

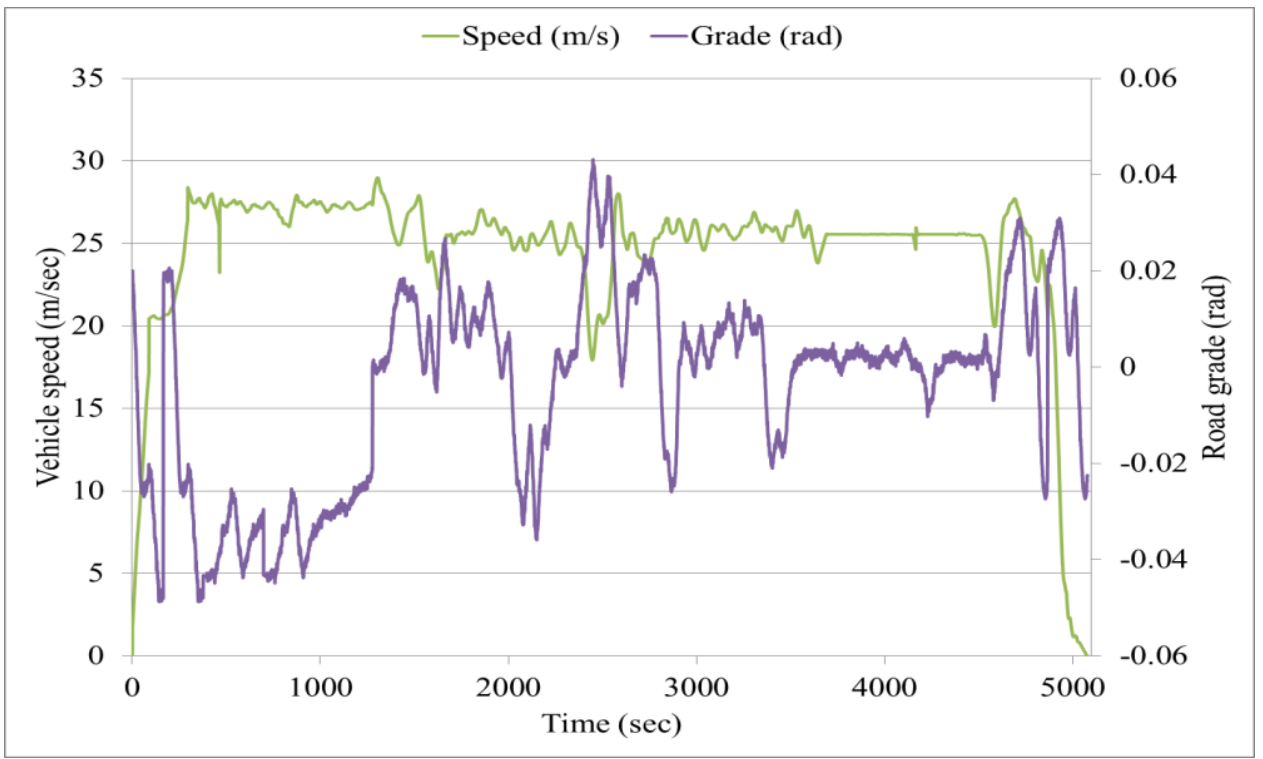

Figure 3.21: Halloran Summit Road, Nipton, CA to Barstow, CA real world driving cycle [56] 


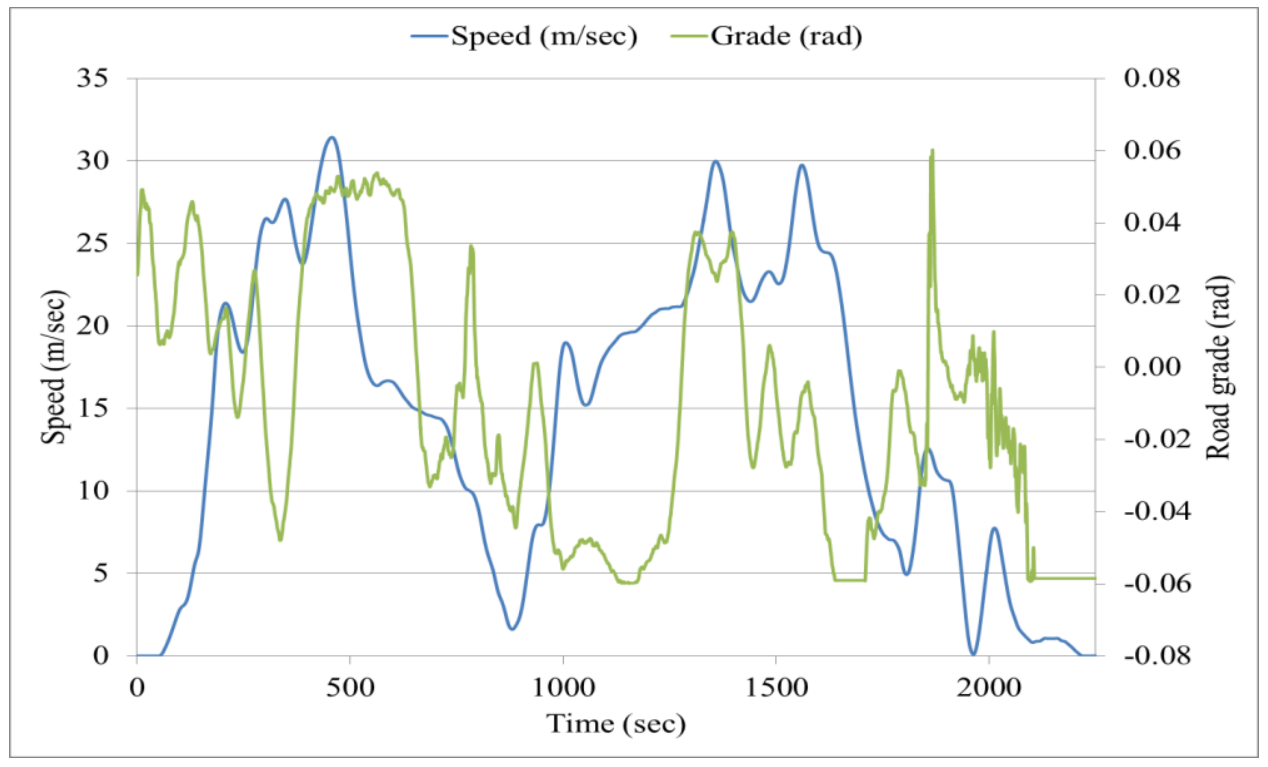

Figure 3.22: Bruceton Mills to Sabraton on I 68, WV driving cycle [56]

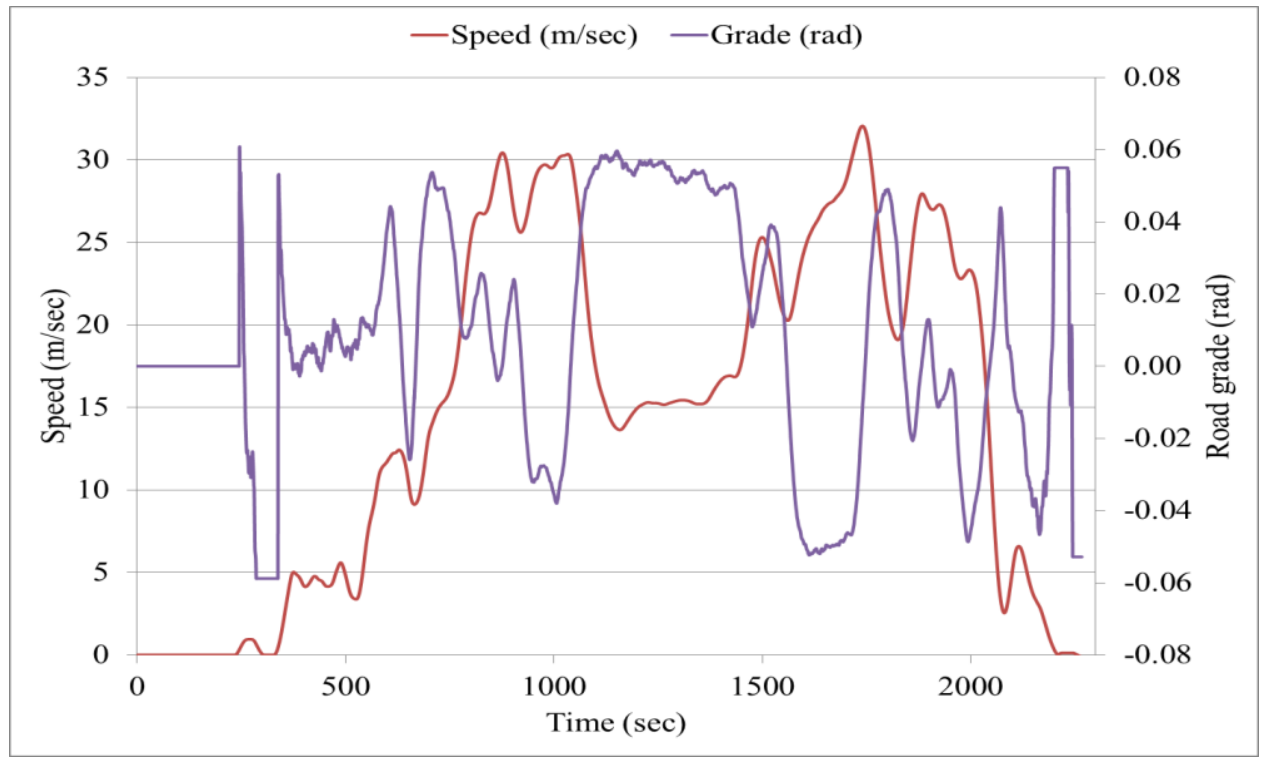

Figure 3.23: Sabraton to Bruceton Mills on I 68, WV driving cycle [56] 


\subsection{Road altitude and grade calculation}

\subsubsection{Road altitude}

The road grade calculation was significant in this study because of the estimated additional power demand required for ascending hills or the available free energy for capture when descending hills. Since the major source for alternative energy for a HEV is using the brake system, road grade needs to be calculated precisely. Barometric pressure was used during the real world cycles to calculate the altitude as a function of pressure and temperature [57]:

$h=\frac{T_{o}}{L}\left(\left(\frac{P}{P_{o}}\right)^{-L R / g}-1\right)$

where: $\mathbf{h}$ represents the altitude (meter), To represents the standard temperature, $\mathbf{R}$ is the universal gas constant for air, $\mathbf{L}$ is the standard temperature lapse rate, Po is the standard pressure, and $\mathbf{g}$ represents gravitational acceleration. The derived equation was based on a subset of the International Standard Atmosphere (ISA) model formulated by the International Civil Aviation Organization (ICAO). The ISA assumed a constant lapse rate between 0 and 33,000 feet altitude [58]. Table (3.5) shows the atmospheric parameters used in the calculation of altitude in SI units.

Table 3.5: Atmospheric parameters [59]

\begin{tabular}{|l|c|c|l|}
\hline Symbol & Value & Unit & Description \\
\hline Po & 101,325 & $\mathrm{~Pa}$ & Pressure at zero altitude (base pressure) \\
\hline To & 288.15 & $\mathrm{~K}$ & Temperature at zero altitude \\
\hline g & 9.80665 & $\mathrm{~m} / \mathrm{s}^{2}$ & Acceleration due to gravity \\
\hline $\mathbf{L}$ & -0.0065 & $\mathrm{~K} / \mathrm{m}$ & Lapse rate \\
\hline R & 287.053 & $\mathrm{~J} / \mathrm{kg} \mathrm{K}$ & Gas constant for air \\
\hline
\end{tabular}




\subsubsection{Road grade calculation}

The American Association of State Highway and Transportation Officials (AASHTO) defined road grade as the rise or fall in vertical distance divided by the horizontal distance for that exact route [59]. To calculate the road grade, geometric relationships were used as shown in Figure (3.24). The change in elevation (V) during a specific time interval $\left(t=t_{2}-t_{1}\right)$ is based on road elevation data, which is the ambient pressure and measured using barometer. The theory of triangles represents the actual distance (S). Equations (3.2-3.5) show the calculation process for road grade.

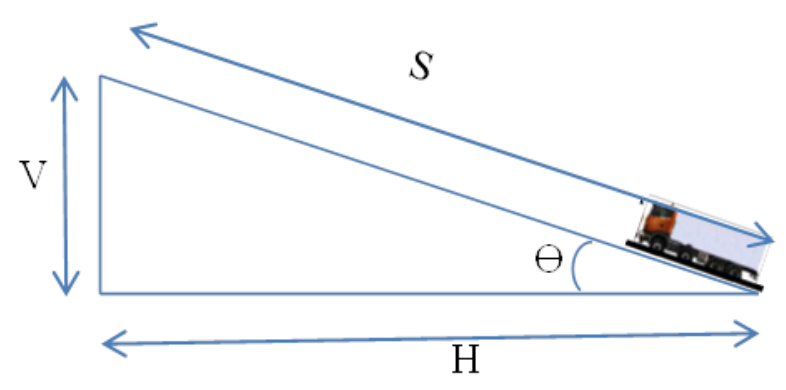

Figure 3.24: Scheme of relationship between

distance and elevation of the road

$S=\bar{v} * t$

Equation 3.2

$H=\sqrt{S^{2}-V^{2}}$

Equation 3.3

$\operatorname{Grade}(\%)=\frac{V}{H} * 100$

Equation 3.4

$\theta=\tan ^{-1}\left(\frac{V}{H}\right)$

Equation 3.5

Some instantaneous calculated grade values were outliers, so the data were post-processed and refined due to signal noise. The refinement was accomplished by removing the outlier values and using linear interpolation between the previous and next value to substitute missing data. 


\subsection{Generating road grade patterns and matching them with the chassis driving cycles such as Transient, Cruise, and HDUDDS:}

Several chassis driving cycles were used to study hybrid electric heavy duty vehicle efficiency [58]. Repeated cycles were performed to simulate a long trip in comparison to chassis driving cycles, which mimics real world conditions. The highway chassis cycles were repeated without stop periods. Several road grades (radian) and oscillation periods $(\mathrm{km})$ were generated. In other words, repeated cruise non-stop driving cycles with different road grades and periodic road grade represented by a sine wave (to mimic hilly and mountain terrain) were used. Repeated HDUDDS driving cycles with different road grades were used here also. Acceleration and deceleration were not detected for these looking-ahead scenarios. Table (3.6) shows the chassis cycles with some properties. Table (3.7) shows the suggested road grades and period. Figures (3.25-3.30) show a sample of different chassis cycles used in this study.

Table 3.6: Different repeated chassis driving cycles

\begin{tabular}{|c|c|c|c|c|c|c|c|}
\hline & Cycle name & Cycle ID & $\begin{array}{l}\text { Ave } \\
\text { Spd }\end{array}$ & $\begin{array}{l}\text { Max } \\
\text { Spd }\end{array}$ & Distance & Duration & $\begin{array}{l}\text { Time } \\
\text { stop }\end{array}$ \\
\hline & & & $\mathrm{mph}$ & $\mathrm{mph}$ & mile & $\mathrm{hr}$. & $\%$ \\
\hline 1 & Cruise cycle & Cruise & 50.97 & 59.06 & 121.62 & 2.39 & 1.89 \\
\hline 2 & Heavy Duty Diesel Truck & HHDDT65 & 60.91 & 66.77 & 136.90 & 2.25 & 1.06 \\
\hline 3 & CARB Transient Cycle & Transient & 15.34 & 47.54 & 17.05 & 1.11 & 18.46 \\
\hline 4 & $\begin{array}{l}\text { HD Urban Dynamometer } \\
\text { Driving Schedule }\end{array}$ & $\begin{array}{l}\text { UDDS } \\
\text { truck }\end{array}$ & 18.74 & 57.66 & 33.12 & 1.77 & 35.28 \\
\hline 5 & $\begin{array}{l}\text { City Suburban Heavy } \\
\text { Vehicle Route }\end{array}$ & CSHVR & 13.59 & 43.80 & 40.32 & 2.97 & 27.70 \\
\hline
\end{tabular}

Table 3.7: Road grades and period generated to match with chassis cycles

\begin{tabular}{|c|c|c|c|}
\hline $\begin{array}{r}\text { Grade\% } \\
\text { Period km }\end{array}$ & \multicolumn{1}{|c|}{$\mathbf{2}$} & \multicolumn{1}{|c|}{$\mathbf{3}$} & $\mathbf{4}$ \\
\hline $\mathbf{5}$ & $2 \% \& 5 \mathrm{~km}$ & $3 \% \& 5 \mathrm{~km}$ & $4 \% \& 5 \mathrm{~km}$ \\
\hline $\mathbf{1 0}$ & $2 \% \& 10 \mathrm{~km}$ & $3 \% \& 10 \mathrm{~km}$ & $4 \% \& 10 \mathrm{~km}$ \\
\hline
\end{tabular}




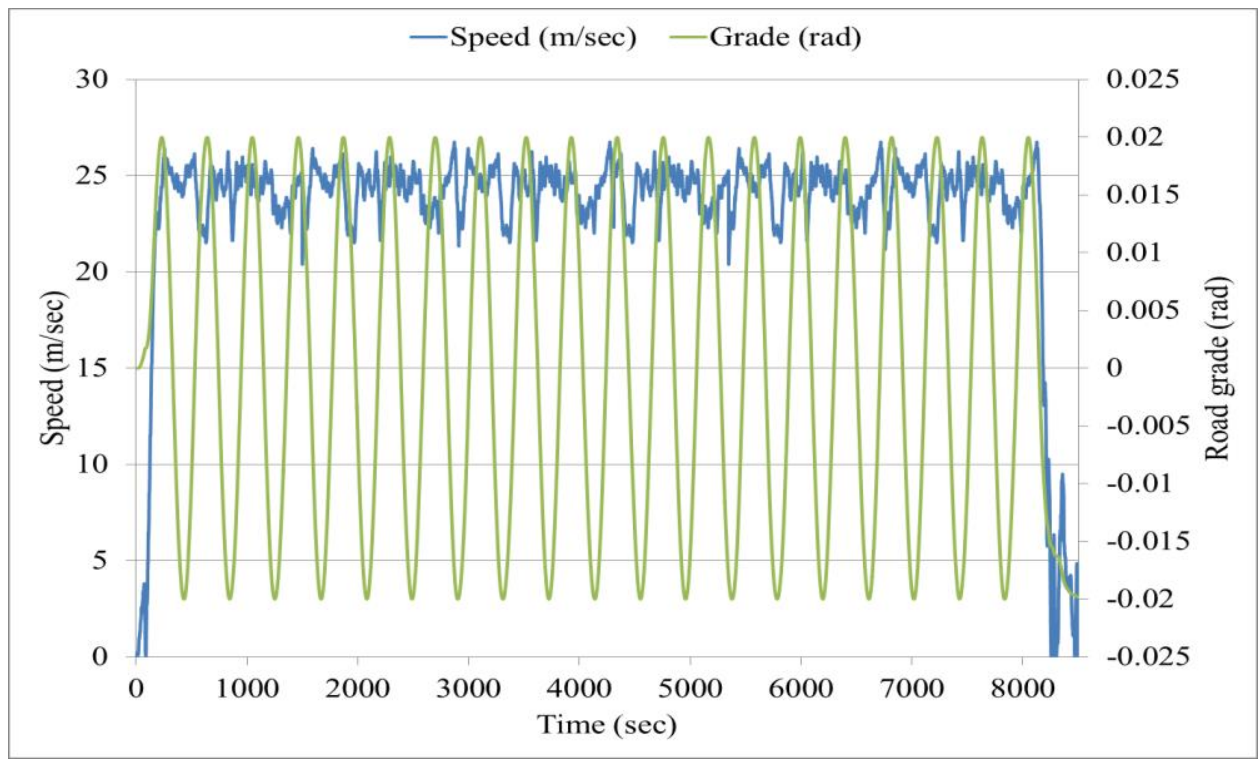

Figure 3.25: Repeated Cruise cycle with $2 \%$ maximum grade and $10 \mathrm{~km}$ period

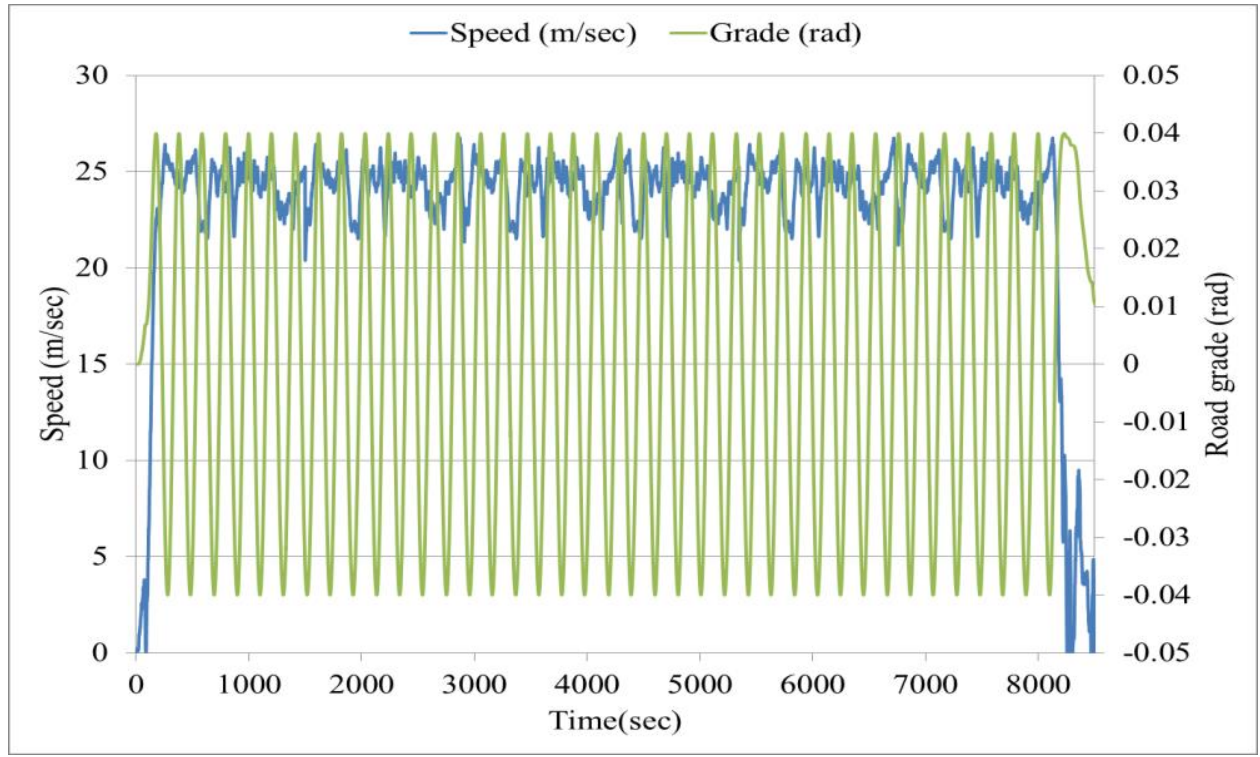

Figure 3.26: Repeated Cruise cycle with $4 \%$ maximum grade and $5 \mathrm{~km}$ period 


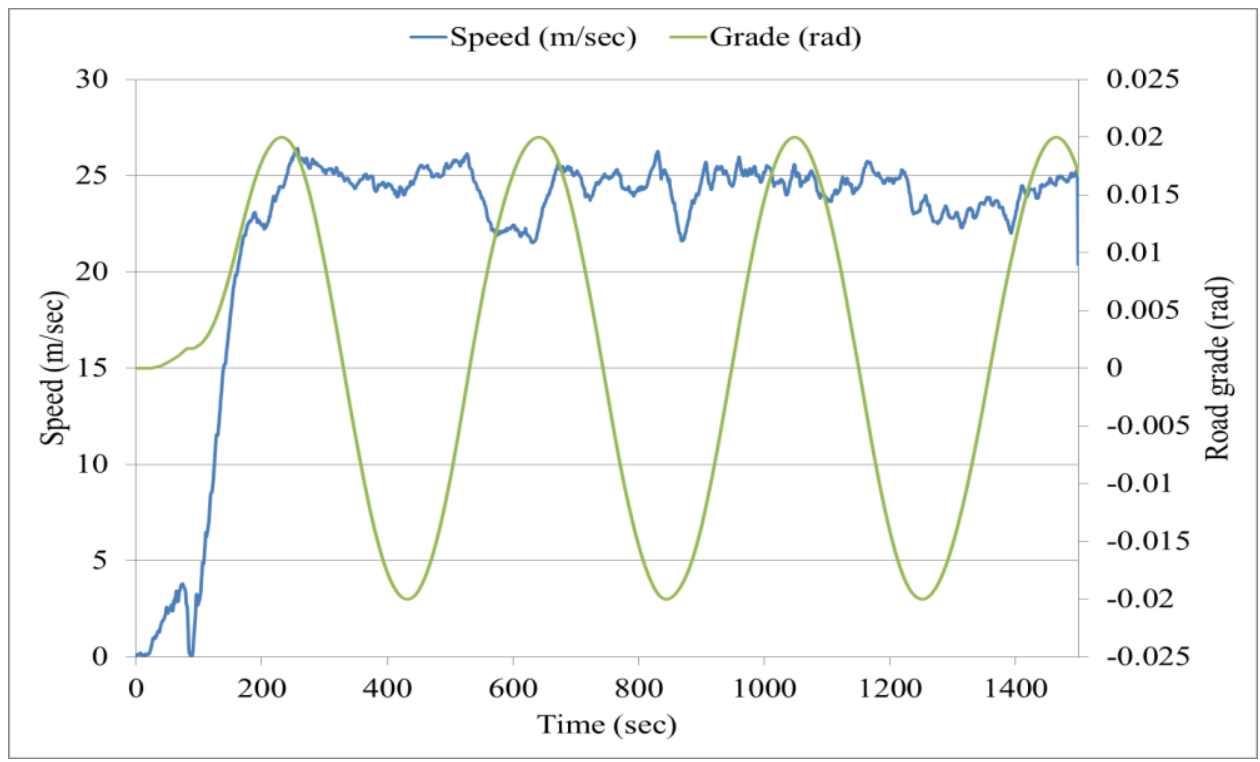

Figure 3.27: Part of repeated Cruise cycle with $2 \%$ maximum grade and $10 \mathrm{~km}$ period

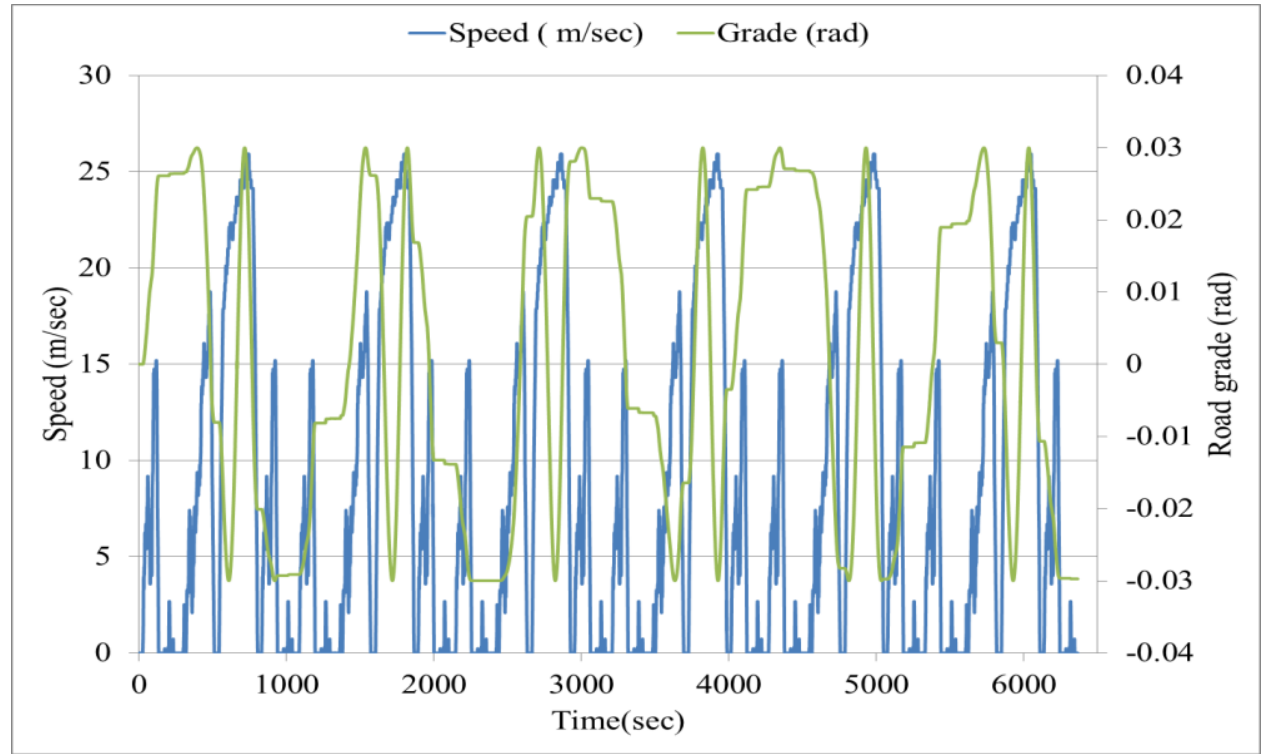

Figure 3.28: Repeated HDUDDS cycle with 3\% maximum grade and $5 \mathrm{~km}$ period 


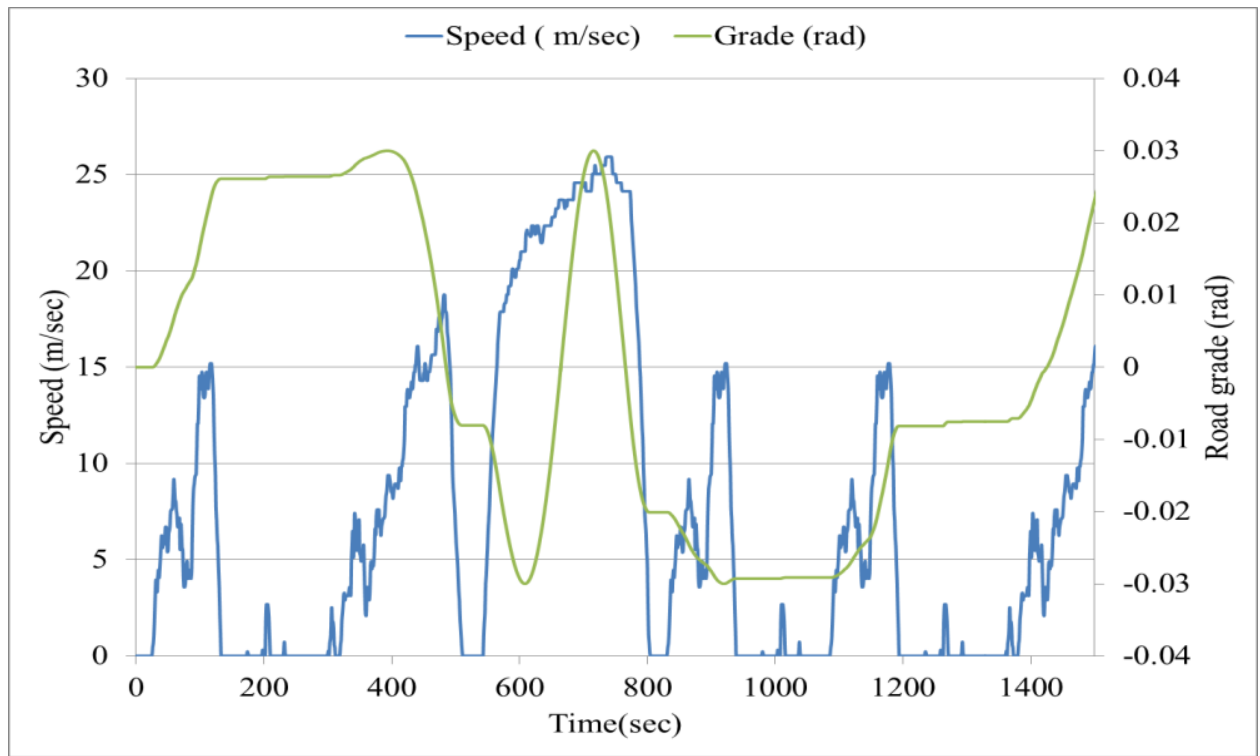

Figure 3.29: Part of repeated HDUDDS cycle with 3\% maximum grade and $5 \mathrm{~km}$ period

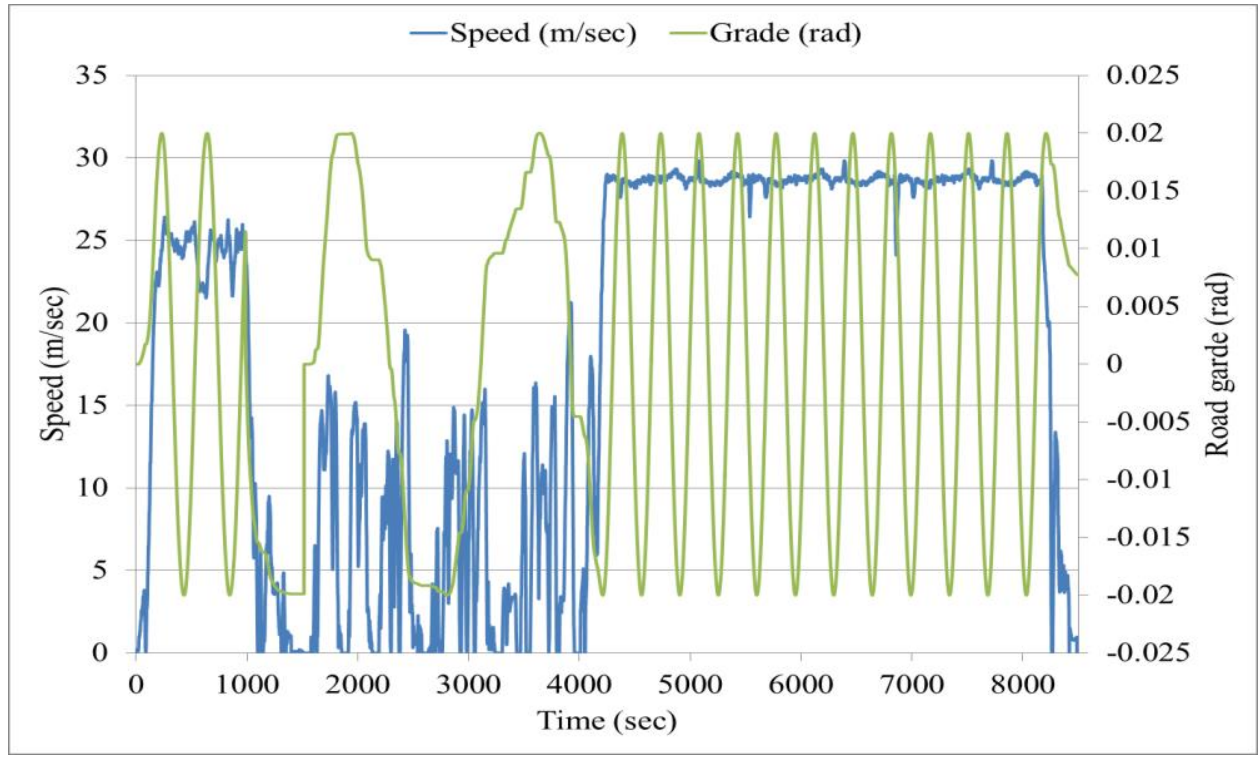

Figure 3.30: Combined Cruise, CSHVR, Transient, and HDT65 cycle with $2 \%$ maximum grade and $10 \mathrm{~km}$ period 


\subsection{Summary}

This section provides an overview of the model. Autonomie has been analyzed to understand the parallel hybrid electric heavy duty vehicle model. The major modification was for the vehicle propulsion controller to enable looking ahead and manage power in advance. The algorithm of the modified model was explained Different real world and chassis cycles were used to evaluate the possible benefits of the parallel heavy duty HEV with looking-ahead control strategy. 


\section{Chapter 4 : The Methodology}

\subsection{Modifying the VPC for a direct parallel HEV model.}

\subsubsection{Conventional vehicle propulsion controller}

For this study the modification will be on the subsystem control strategy to calculate the engine torque demand. The conventional calculation of the engine torque demand worked by inputting the parameters (wheel torque demand, wheel speed demand, SOC, accessories power, engine speed, and maximum engine torque). The target SOC was sustained at the middle. If the battery SOC is above the middle, then the ESS would be depleted. On the other hand, if the battery SOC was below middle, then the ESS would be charged. Accessory power was added to the total power demand. If the total torque demand was surpassed the maximum engine torque, then the maximum engine torque was commanded as the output engine torque. For the VPC, if the wheel power demand exceeded the output engine power, then the VPC depleted the ESS regardless of the battery threshold SOC, as long as battery SOC was above the minimum safety limit and motor power was capable of assisting the main power source. For this study, the ESS's power was assumed equal to the motor's power. Figure (4.1) shows diagram of the calculation sequence for engine torque demand [54].

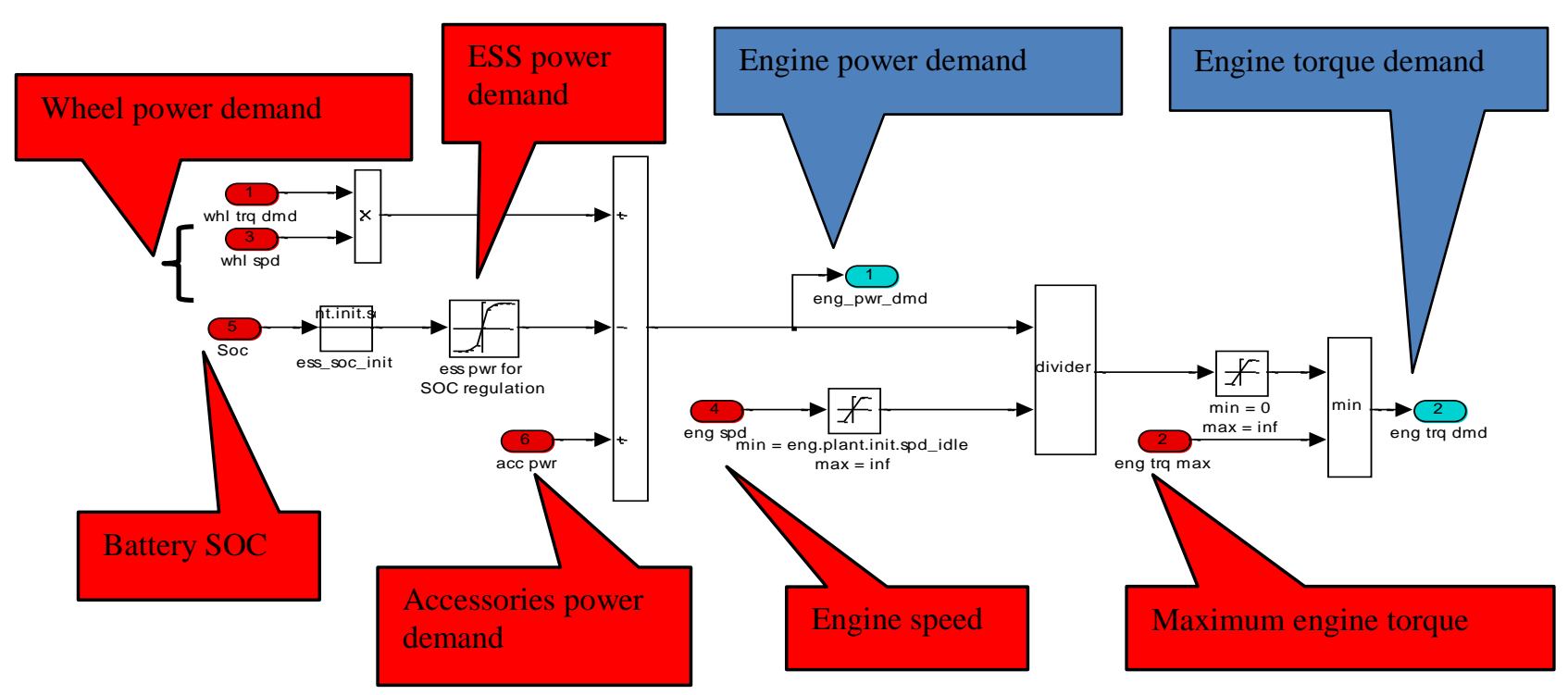

Figure 4.1: Subsystem control strategy for a direct parallel pre-transmission HEV to calculate engine torque demand [54] 


\subsubsection{Modified vehicle propulsion controller}

The modified subsystem control strategy to calculate engine torque demand for a looking-ahead strategy worked basically by opening a time window in the future, calculating the power demand. Based on that calculation, the engine torque demand was recalculated to manage the battery SOC. Figure (4.2) shows the diagram of the proposed looking-ahead subsystem control strategy to manage the battery SOC and calculate the new engine torque demand.

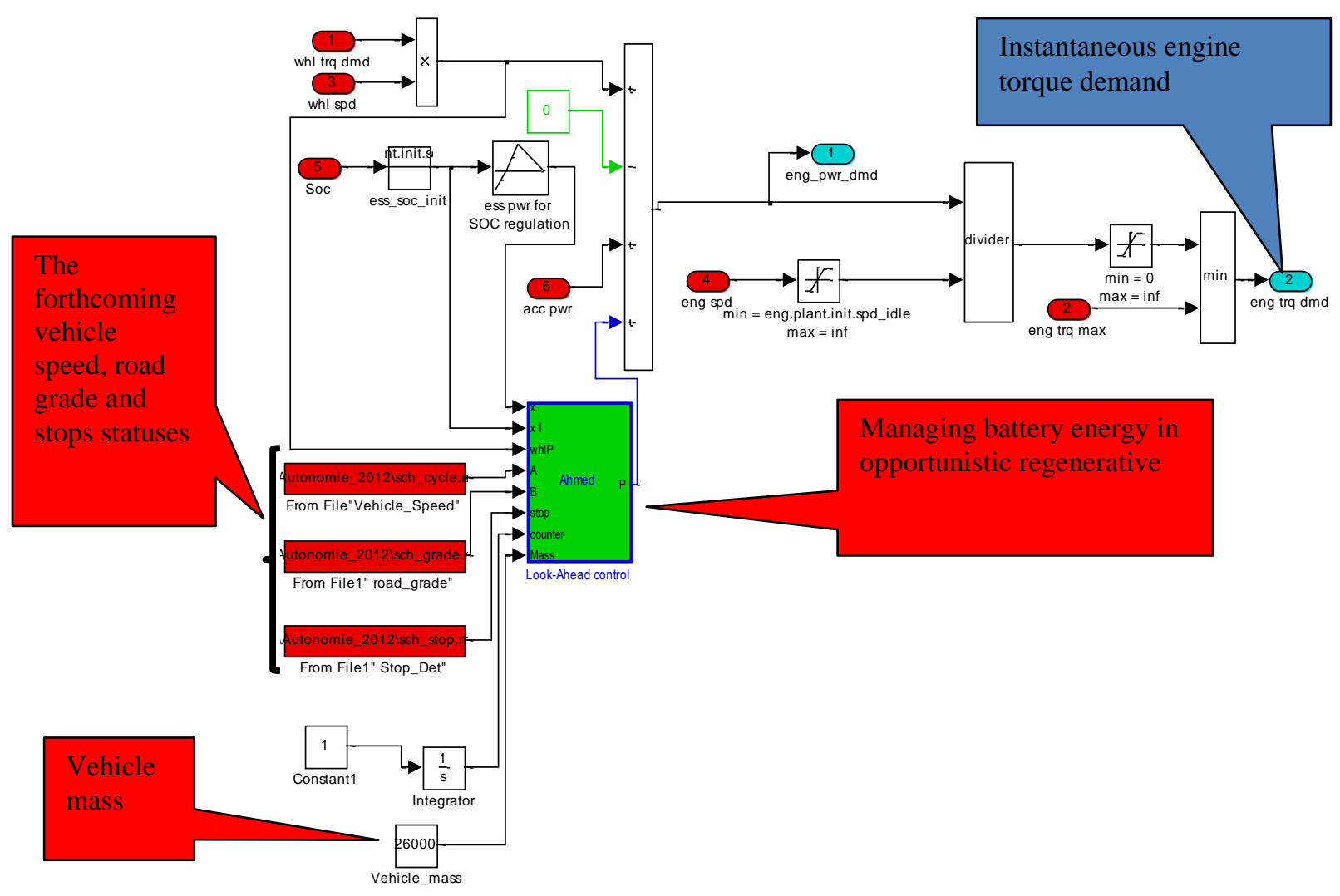

Figure 4.2: Diagram of the proposed looking-ahead subsystem control strategy for a direct parallel pre transmission HEV to manage the battery SOC 


\subsection{Algorithm of the modification on vehicle propulsion controller}

\subsubsection{Simple algorithm}

Following is the algorithm for the looking-ahead model to manage the battery SOC and calculate the new instantaneous engine torque based on the forthcoming power demand. For the modified controller, if the instantaneous vehicle speed was less than $25 \mathrm{mph}$ (city activity) and the electric motor power was sufficient to propel the wheels, then the vehicle was driven as an electric vehicle (engine off). Moreover, the $25 \mathrm{mph}$ speed was selected to switch between electric modes to hybrid mode because it represents a speed limit in most cities' routes, and it could be tuned if needed.

1- Open a time window (one minute) in the future and calculate the average speed ahead and compare it to the current speed for the vehicle.

2- Simultaneously for the same opened window, calculate the average forthcoming road grade and compare it to the current road grade.

3- Use the road load equation to calculate the negative road grade when the vehicle starts using the brake system as shown in equation 4.1 .

$P_{t}=\left(m \frac{d v}{d t}+\frac{1}{2} C_{D} \rho A v^{2}+\mu \mathrm{mg}+\mathrm{mg} \sin (\theta)\right) v$

Equation 4.1

Equation 4.2 assumes that the vehicle propulsion power demand becomes zero before it starts becoming negative, and it assumes that the vehicle is moving at constant speed (i.e. using the cruise control system, and the acceleration and deceleration $=0$ ).

Assume $P_{t}=0$ and $\frac{d v}{d t}=0$.

To calculate negative road grade (radian) ahead considering vehicle speed and mass as variables, the equation was as follows:

$\theta=\sin ^{-1}\left(\left(-\frac{1}{2} C_{D} \rho A v^{3}-\mu \mathrm{mgv}\right) /(m g v)\right)$

Equation 4.2 
4- If the road grade ahead is less than calculated negative road grade (equation 4.2), then calculate the expected power ahead and manage the battery SOC to guarantee enough battery capacity to capture the upcoming free energy (from using the brake system) as much as possible.

5- If the road grade ahead is greater than zero, then calculate the power needed to go uphill. Then if the power demand to go uphill will surpass $80 \%$ of engine peak power (to give a chance for un expected acceleration and better fuel efficiency also [57]), then charge the battery the same as the energy demand ahead if the battery does not have enough energy for that energy demand ahead.

6- For both charging or discharging status, if the equipment has not generated enough power to respond, then the controller will make them work at maximum possible working zone considering all limitations to simulate the vehicle performance in real life as Autonomie software is doing.

7- This procedure keeps repeating every second during the trip to manage battery SOC in opportunistic regenerative braking.

8- The engine power management works to guarantee that the power from the engine and the battery together will be adequate for the next event to the extent possible.

9- The looking-ahead strategy stops executing 10 minutes ahead of the trips' ends, so that the cycle ends with the same battery SOC as for without the looking-ahead strategy as possible.

10- The communication between the infrastructures to vehicle (I2V) is assumed to be continuous, and the information will be updating regularly every two minutes.

\subsubsection{Sophisticated algorithm}

For a sophisticated control strategy, it was found that significant improvement can be achieved by opening a dynamic window within the time window that is already open. The purpose of the dynamic window is to calculate the cumulative energy needed or available, and determine if the battery SOC will be critical. Then, based on that calculation, the VPC will make a decision to manage battery SOC in opportunistic regenerative braking. Moreover, if no opportunity to charge the battery ahead of a climb is available, then the fuel energy will be used increase the SOC. The major benefit is to avoid unnecessary charging or discharging efficiency losses. Figure (4.3) shows the scheme for expected forthcoming traffic conditions to make a proper action in 
advance. The symbols $\mathbf{x}$ and $\mathbf{y}$ in the Figure refer to the beginning and the end of the lookingahead period (window time). Real world and chassis dynamometer driving with road grades for city and highway driving cycles were used to study the possible impact of using ITS information on parallel heavy duty HEV efficiency.

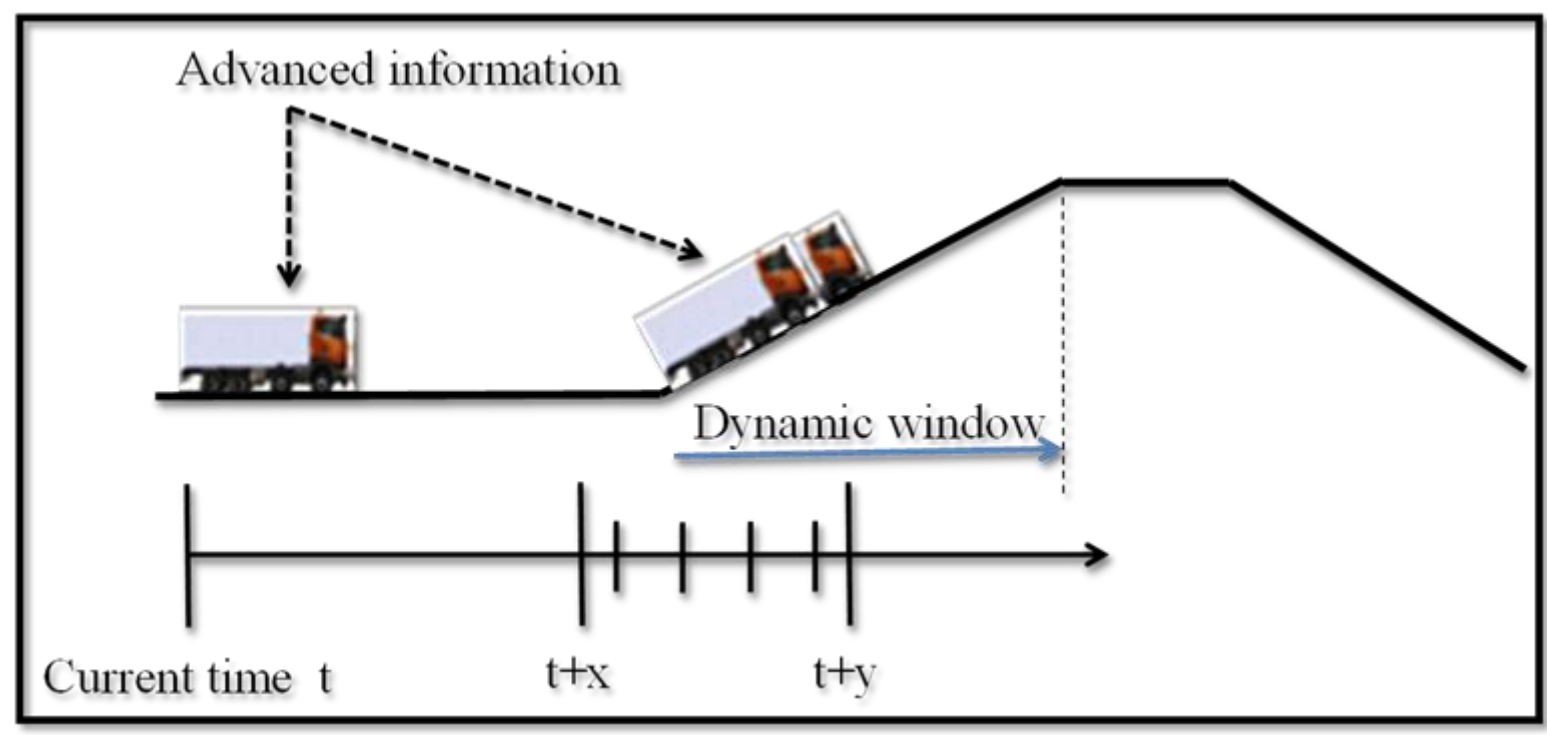

Figure 4.3: Scheme of looking-ahead strategy for forthcoming traffic conditions.

\subsection{Scenarios to study the impact of ITS information ahead.}

At the start of each trip with a known destination, a GPS is going to generate a speed profile based on infrastructure information for ITS and generate the road grade profile from the database for GIS. The main concern of looking-ahead strategy is obtaining false or inaccurate information for the forthcoming traffic condition. When the information for forthcoming traffic conditions is wrong, the impact might be worse than without looking-ahead strategy. This study assumed different scenarios of the information precision and details that the VPC might receive to manage ESS in opportunistic regenerative.

Five scenarios are proposed for this study as following:

1- Scenario 1 (Sc1): ITS information ahead will be for forthcoming route elevation with $\pm 1.5 \%$ road grade resolution, practical vehicle speed, and opening one window each time.

2- Scenario 2 (Sc2): ITS information ahead will be for forthcoming practical route elevation, speed limit, and opening one window each time. 
3- Scenario 3 (Sc3): ITS information will be regarding route elevation, practical speed ahead with $\pm 5 \mathrm{mph}$ resolution, and opening one window each time.

4- Scenario 4 ( $\mathrm{Sc} 4)$ : ITS information will be regarding route elevation, practical speed ahead with $\pm 10 \mathrm{mph}$ resolution, and opening one window each time.

5- Scenario 5 (Sc5): ITS information ahead will be regarding route elevation, speed limit, practical speed, and stop detection. In this scenario two windows at the same time will be open, one window for a short time ahead, such as 1 minute ahead, to detect the vehicle stopping and second time window for a longtime, such as 3, 5, and 7 minutes ahead (based on the hybridization equipment sizes), ahead regarding route elevation and practical speed.

Therefore, these scenarios can help to illustrate the possible positive and negative impacts of forthcoming information precision. In other words, the false alert for power demand or driver using the brake system can impact negatively on fuel economy or alternative power contribution. The term practical vehicle speed used in the scenarios means the real time vehicle speed ahead. Moreover, the plus/minus traffic information accuracy is generated by assuming that the data accuracy is switching every 50 seconds. For example, $\pm 10 \mathrm{mph}$ resolution means that the forthcoming real time vehicle speed increased by $10 \mathrm{mph}$ for 50 seconds and then decreased by $10 \mathrm{mph}$ for the next 50 seconds and so on.

\subsection{Hybridization equipment efficiency}

The hybridization equipment consist mainly of the electric motor, energy storage system and vehicle propulsion controller. Electric motor efficiency represents the main source for energy losses [60]. Energy storage system represents another loss factor due to charging and discharging efficiency [46].

\subsubsection{Energy storage system efficiency}

The hybrid electric vehicles are available in the auto market and passed the first phase, which is the concern of architecture sophistication and maintenance issues [61]. The customers' demand is concerned with improving HEV performance. One of the main sources for energy losses in HEV is the energy storage system losses. The ESS efficiency is a function of the current and internal resistance of the battery, which cause loss. Ni-MH battery showed a strong trend in low heat production during charging and discharging [62]. Nickel metal hydride battery, which is 
used in this study, may help to improve HEV in two ways: the first improvement is decreasing the ESS cost, and the second improvement is the ESS efficiency. Figure (4.4) shows the efficiency for ESS on heavy duty Transient driving cycle for heavy duty HEV model using Autonomie software [54].

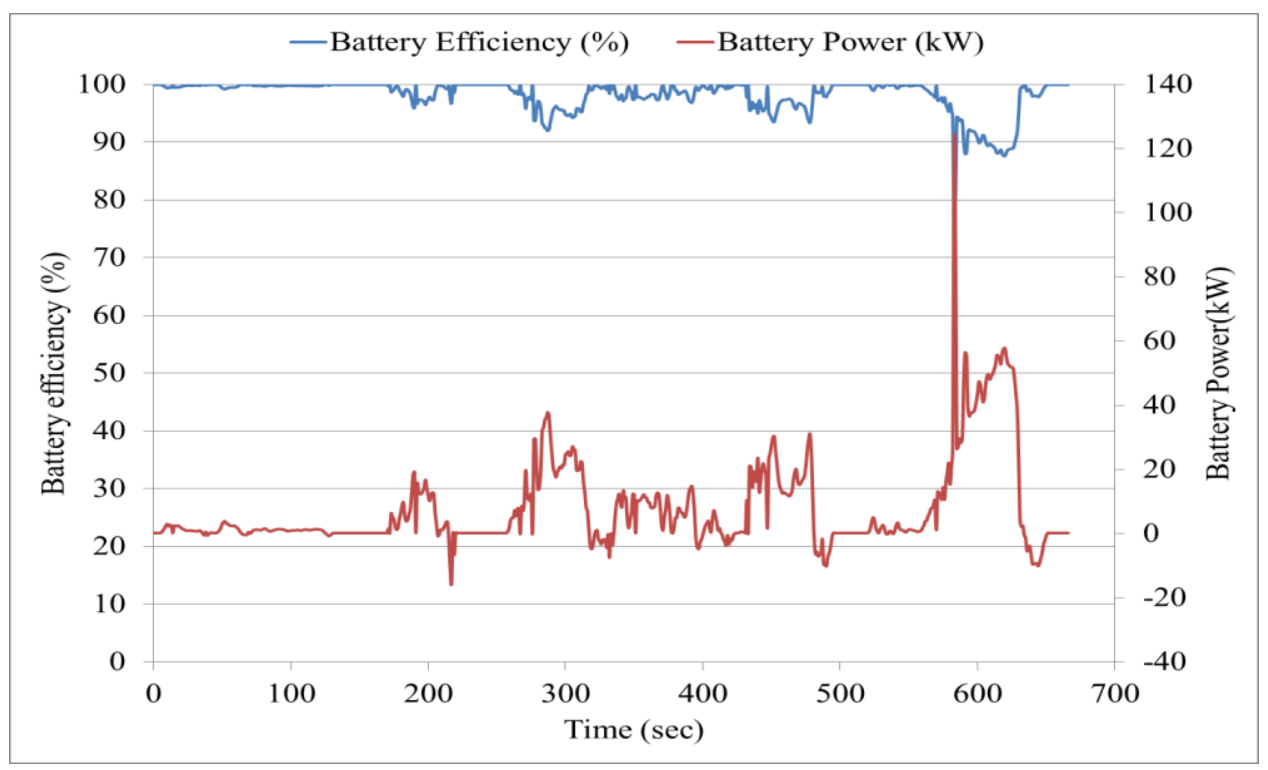

Figure 4.4: Energy storage system efficiency and power on heavy duty transient driving cycle

\subsubsection{Electric motor efficiency}

The electric motor efficiency represents a major source to decrease the ESS charging and discharging total efficiency [63]. Manufacturers are working continuously to improve HEV equipment efficiencies including electric motors [63]. UQM developed a technology to provide the market with efficient electric motors for HEV specifically [64]. UQM Power Phase electric motors which are used in this study show a strong trend regarding the efficiency and performance $[64,65]$. The design characteristics of the electric machine contribute to lower usage of copper, iron and other materials, and it contributes to reduced built-up costs compared to other motors of similar power. These aspects have permitted them to rate their advanced motors and controls competitively while providing better performance than conventional motors and controllers. UQM motors can be operated in either a forward or reverse direction of rotation, or it can be operated either in motor or generator mode with the ability to dynamically change from one mode of operation to another in millisecond response time [64, 66]. On the other hand, 
the electric motors have some limits and that is due to thermal issues; for example the electric motor used in this study cannot run at the maximum power more than 250 seconds [54]. A UQM electric motor has been used for this study. Figures (4.5 and 4.6) show the electric motor efficiency.

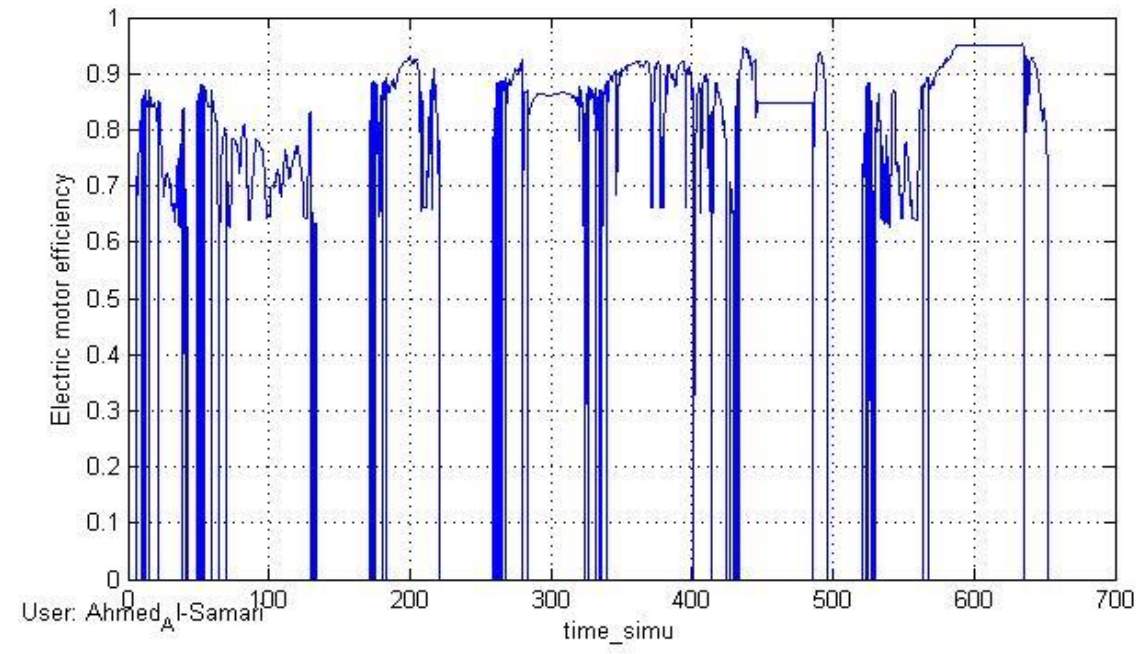

Figure 4.5: Electric motor efficiency [56]

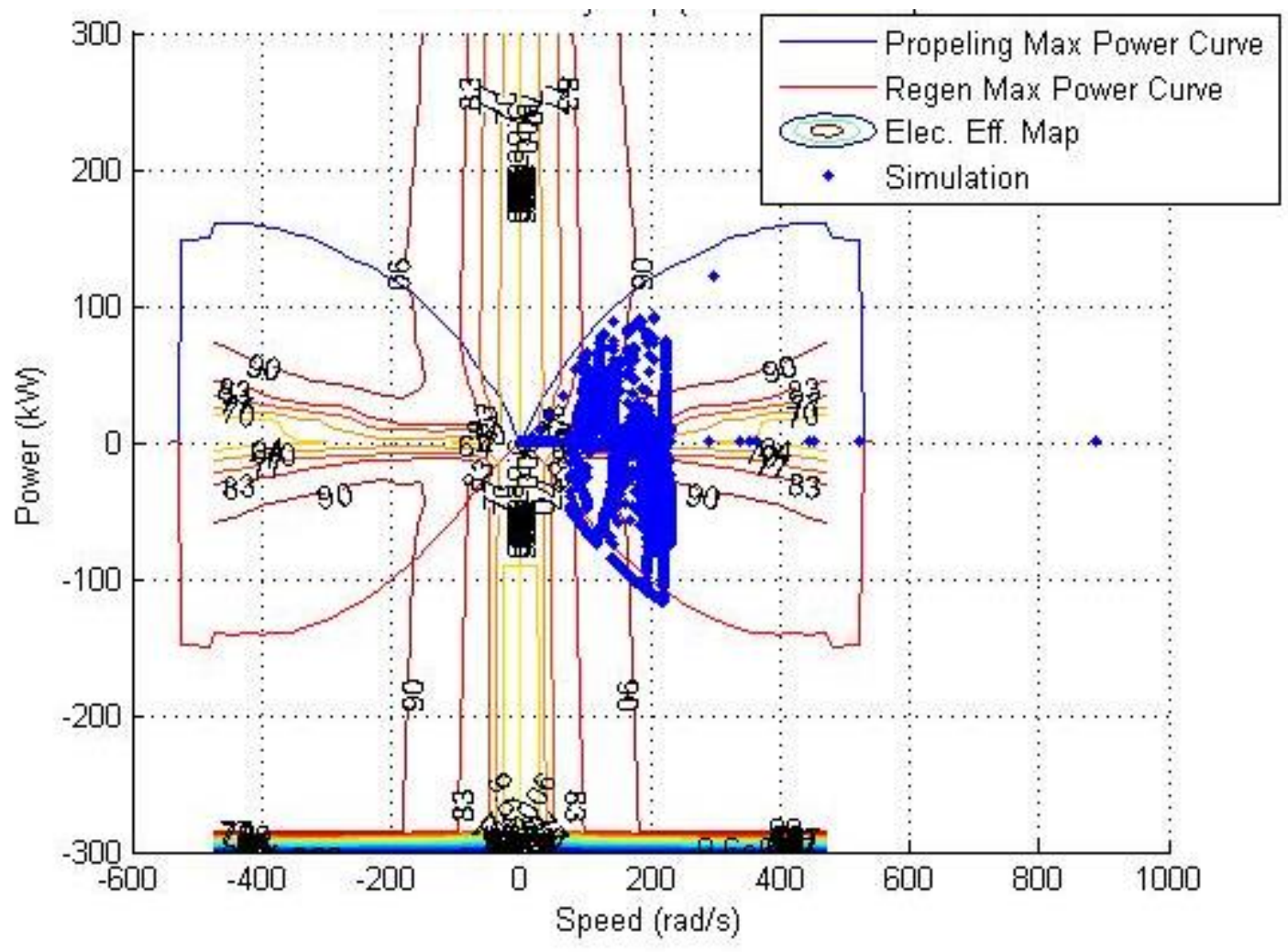

Figure 4.6: Electric motor efficiency map for $150 \mathrm{~kW}$ motor power [56] 


\subsection{Calculation of time needed for charging and discharging electric storage system}

The time needed for charging or discharging the energy storage system was calculated based on the equipment capacity. Moreover, the electric motor may work at $70-80 \%$ of peak load to work at better efficiency according to the efficiency map [67]. For the calculated ESS and electric motor efficiency in the previous sections, the rough estimation of charging and discharging efficiency could be assumed as $80 \%$ for the electric motor and $95 \%$ for the battery pack. On the other hand, during the simulation, equipment efficiency will be calculated more accurately based on their efficiency maps using Autonomie software [54].

The maximum effective capacity of the battery pack to be charged or discharged represents about $55 \%$ of the battery pack size. The battery cannot be discharged below the safety limit, which is $30 \%$, and cannot exceed 90\% [46]. On other hand, since the ahead energy estimation is based on averaging the information (vehicle speed and road grade) ahead, it will be more convenient to give a tolerance with $5 \%$ of SOC, for small events might not be expected, such as driver unexpected demand for acceleration or deceleration. In other words, the modified VPC was limiting charging of the battery to a maximum of $85 \%$ SOC and discharge to a minimum of $35 \%$ SOC based on forthcoming events. Equation 4.3 is used to calculate time needed for charging and discharging the energy storage system. Figure (4.7) shows the time required for charging and discharging with different equipment sizes.

Time $_{(\text {minutes for charge or discharge })}=\frac{60 * 0.55 * \text { Battery energy size } / 0.95}{\text { motor power } * 0.8} \quad$ Equation 4.3 


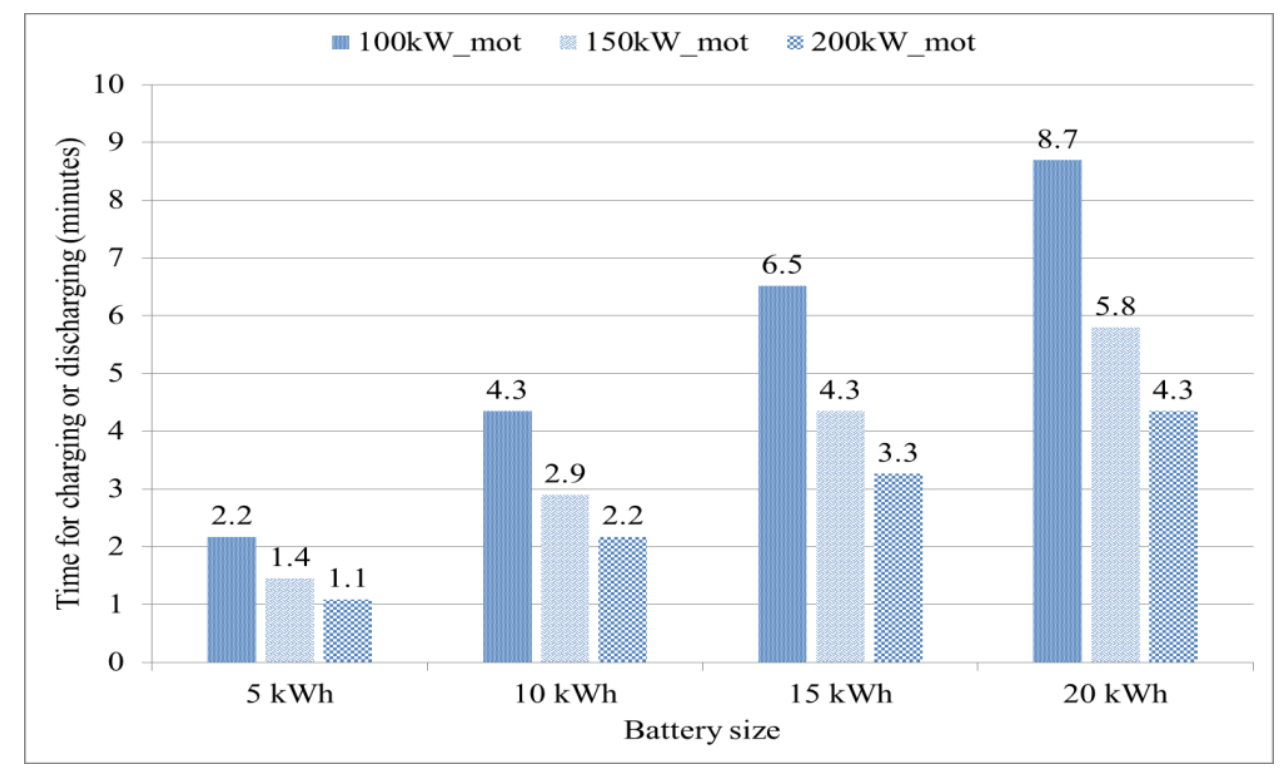

Figure 4.7: Charging and discharging time for different energy storage system sizes

\subsection{Estimate the negative road grade when driver needs to depress the brake}

The method used to predict the forthcoming energy demand was by applying the road load equation to calculate the total power demand. The negative road grade was calculated based on two assumptions; the first was that the power demand will become zero (power demand decreased to become zero, and then it starts becoming negative); the second assumption was that the acceleration and deceleration will be zero. Equation 4.4 shows the negative road grade when the driver start using brake system.

$\theta=\sin ^{-1}\left[\left(-\frac{1}{2} C_{D} \rho A v^{3}-\mu \mathrm{mg} v\right) /(m g v)\right]$

Equation 4.4

Where, $\mathbf{m}$ is mass of the vehicle $(\mathrm{kg}), \boldsymbol{v}$ is speed of the vehicle $(\mathrm{m} / \mathrm{sec}), \mathbf{A}$ is frontal area of the vehicle $\left(2.25 \mathrm{~m}^{2}\right.$ and $7.5 \mathrm{~m}^{2}$ for light and heavy duty, respectively), $\mathbf{g}$ is acceleration due to gravity $\left(9.81 \mathrm{~m} / \mathrm{sec}^{2}\right), \mathbf{C}_{\mathbf{D}}$ is aerodynamic drag coefficient $(0.79$ and 0.3 for heavy duty and light 
duty respectively $[68,69]), \boldsymbol{\mu}$ is tire rolling resistance coefficient $(0.008$ and 0.00938 for light and heavy duty vehicle respectively $[69,70]), \boldsymbol{\rho}$ is air density, and $\boldsymbol{\theta}$ is the negative road grade to start using brake system in radian ( $\mathrm{rad})$.

Figures (4.8 and 4.9) show the variation of negative road grade relative to vehicle weight and speed to start using the brake system for heavy and light duty vehicles respectively. It can be noticed that the sensitivity of heavy duty for negative road grade is significantly more than light duty vehicles for negative road grade. For example, the light duty driver at weight and speed of $1,000 \mathrm{~kg}(2,200 \mathrm{lb})$ and $25 \mathrm{~m} / \mathrm{sec}(56 \mathrm{mph})$, respectively, will use the brake system when negative road grade is less than $-3.6 \%$. However, for heavy duty truck with $25,000 \mathrm{~kg}(55,000 \mathrm{lb})$ and $25 \mathrm{~m} / \mathrm{sec}(56 \mathrm{mph})$, a driver will need to use brake system when the negative road grade is less than $-1.9 \%$. Therefore, the heavy duty drivers need to use the brake system more frequently than light duty drivers to maintain vehicle speed.

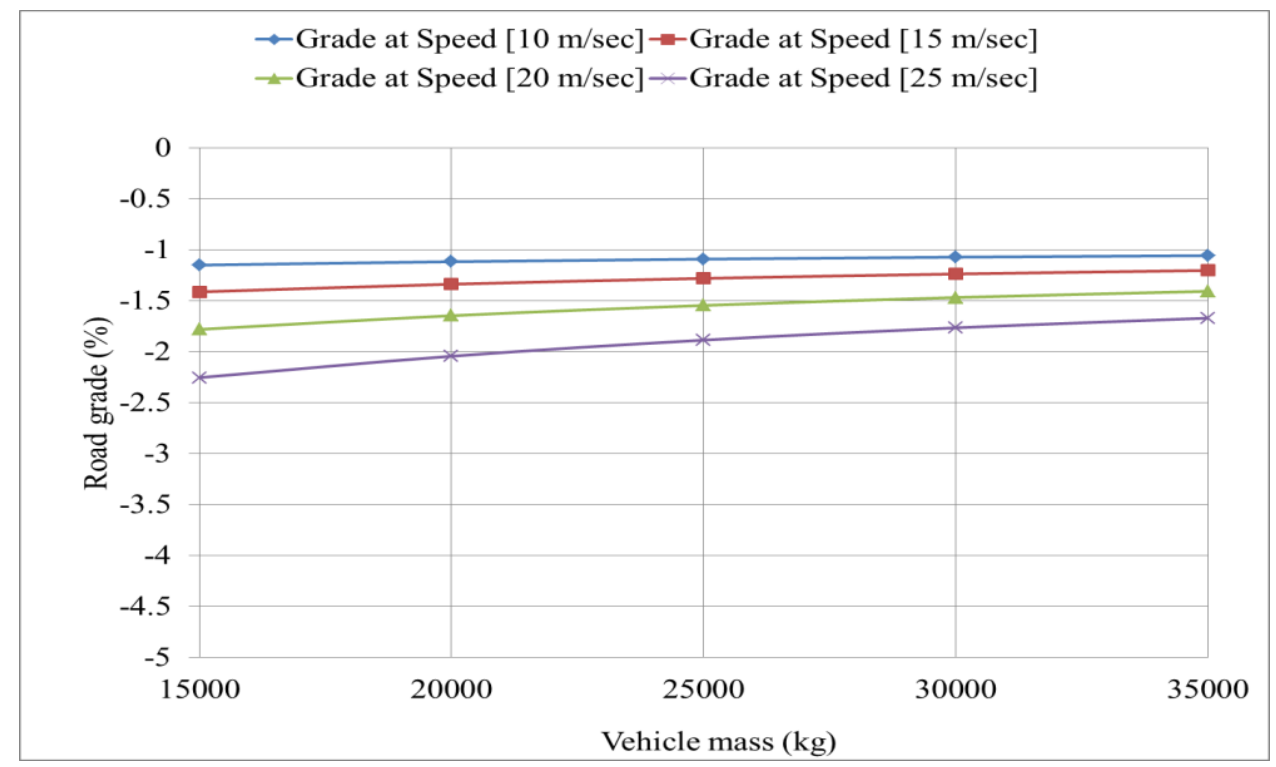

Figure 4.8: Negative road grade to start using brake system relative to vehicle speed and weight for heavy duty vehicle 


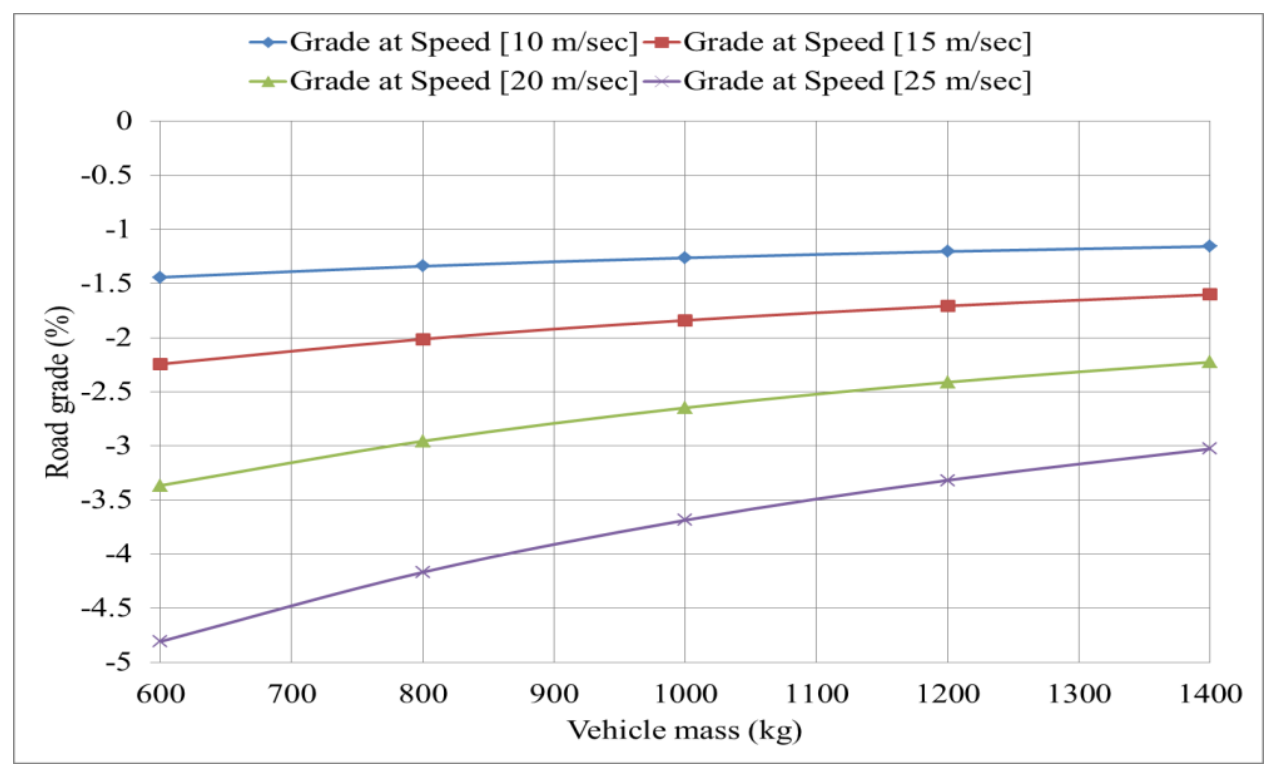

Figure 4.9: Negative road grade to start using brake system relative to vehicle speed and weight for light duty vehicle

\subsubsection{Energy expected using the brake system for truck in comparison to light duty}

The free energy expected from using the brake system, when descending hills, is proportional mainly to the vehicle mass and speed as shown in the road load equation. Figures (4.10 and 4.11) show the negative power that could be available at the wheel to capture for heavy and light duty vehicles. For the same negative road grade and speed, the power expected using the brake system for a truck is much more than the power expected from a car. Whereas the typical engine sizes are about $160 \mathrm{hp}$ and $500 \mathrm{hp}$ for a car and a truck, respectively, the power available from using the brake system was significantly different. This amount of free energy available at wheels have several limitations to store it in the ESS, such as electric generator peak and continuous current limits, the battery charging efficiency, and also the ESS peak and continuous charging control limits. This free energy to capture using the brake system, when the vehicle was descending a hill, encourages optimizing the VPC to capture as much free energy as possible. This optimization includes the looking-ahead control in cooperation with the ITS information. 


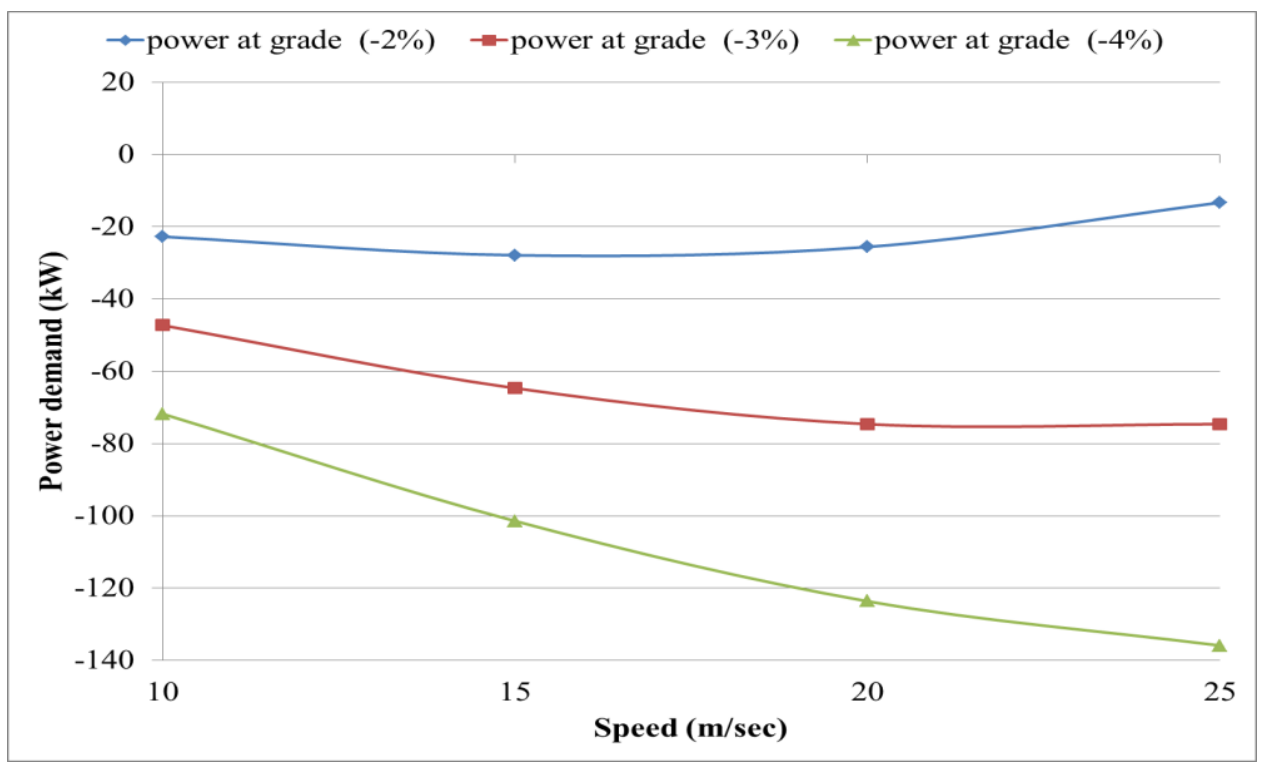

Figure 4.10: Power demand at wheels for truck $(25,000 \mathrm{~kg})$ at different negative road grade

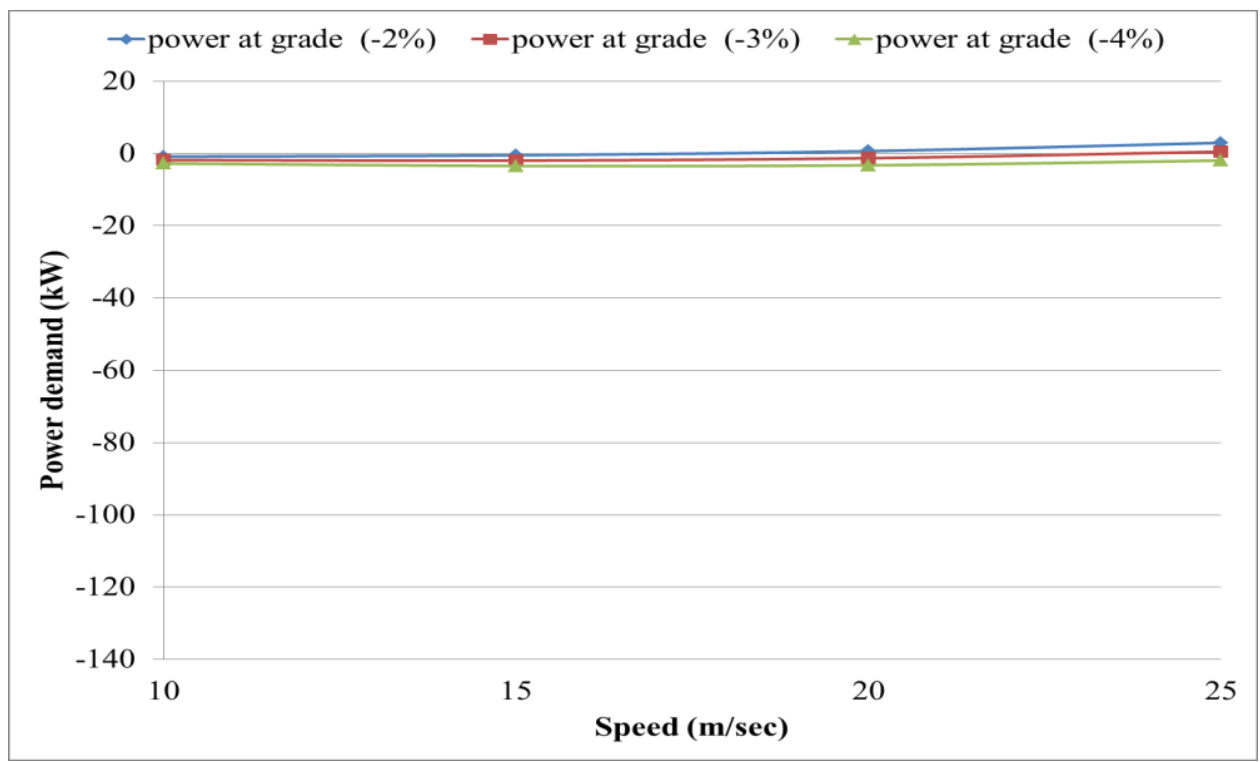

Figure 4.11: Power demand at wheels for light duty vehicle $(1000 \mathrm{~kg})$ at different negative road grade 


\subsubsection{Impact of aerodynamic and tire improvement on increasing use of the brake}

The vehicle manufacturing technologies are improving continuously and the chance to improve vehicle performance through decreasing power losses are increasing. Decreasing rolling resistance and air drag coefficients can contribute to decreasing the total power demand to propel a vehicle according to the road load equation. Moreover, decreasing the coefficients can impact the sensitivity of vehicles to the negative road grade which makes the driver start using the brake system to compromise vehicle speed earlier. Therefore, hybrid electric vehicle contribution comes in capturing that expected increased free energy. Most indicators indicate that the hybrid electric vehicles are going to contribute significantly in improving fuel economy and decreasing greenhouse gases emissions. So, any improvement in the hybrid electric vehicles performance can positively impact the consumers.

Figure (4.12) shows the impact of decreasing rolling resistance coefficient by $25 \%$ ( 0.00938 to 0.007035) on the sensitivity for the negative road grade to make drivers start using the brake system for trucks. Figure (4.13) shows the impact of decreasing the aerodynamic drag coefficient by $25 \%$ ( 0.79 to 0.59 ) on sensitivity for negative road grade to make the driver start using the brake system. Moreover, Figure (4.14) shows the impact of decreasing both rolling resistance and aerodynamic dynamic drag coefficients by $25 \%$ on the sensitivity for negative road grade that make drivers start using the brake. The results of calculations revealed that decreasing of rolling resistance by $25 \%$ can increases the chance for drivers to use the brake (negative road grade change) by about $20 \%$ at low speed $(25 \mathrm{mph})$. This percentage decreases as vehicle speed increasing until about $6 \%$. On the other hand, decreasing of air drag coefficient by $25 \%$ can increase the chance for using the brake (change negative road grade) by about $10 \%$ at high speed (60 mph). This chance decreases as vehicle speed decreases until about 3\% at low speed (25 $\mathrm{mph}$ ). When considering decreasing rolling resistance and air drag coefficients, the chance for drivers to use the brake system is increased by about $23 \%$ at different vehicle speed. In the Figures, $\mu 1$ and $\mathrm{Cd} 1$ represent the original values of rolling resistance and air drag coefficients respectively. The $\mu 2$ and $\mathrm{Cd} 2$ represent the new values with a $25 \%$ decrease. 


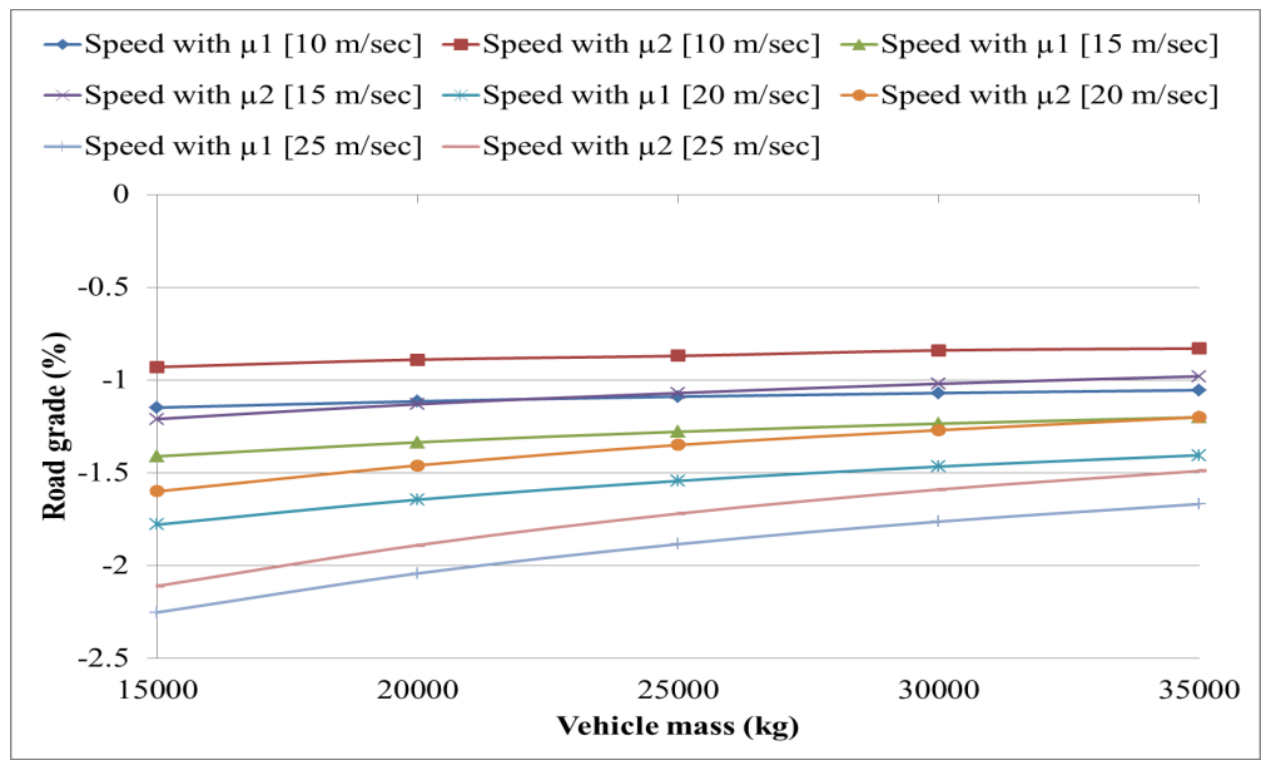

Figure 4.12: Negative road grade when driver needs to start depressing brake with different rolling resistance coefficient values

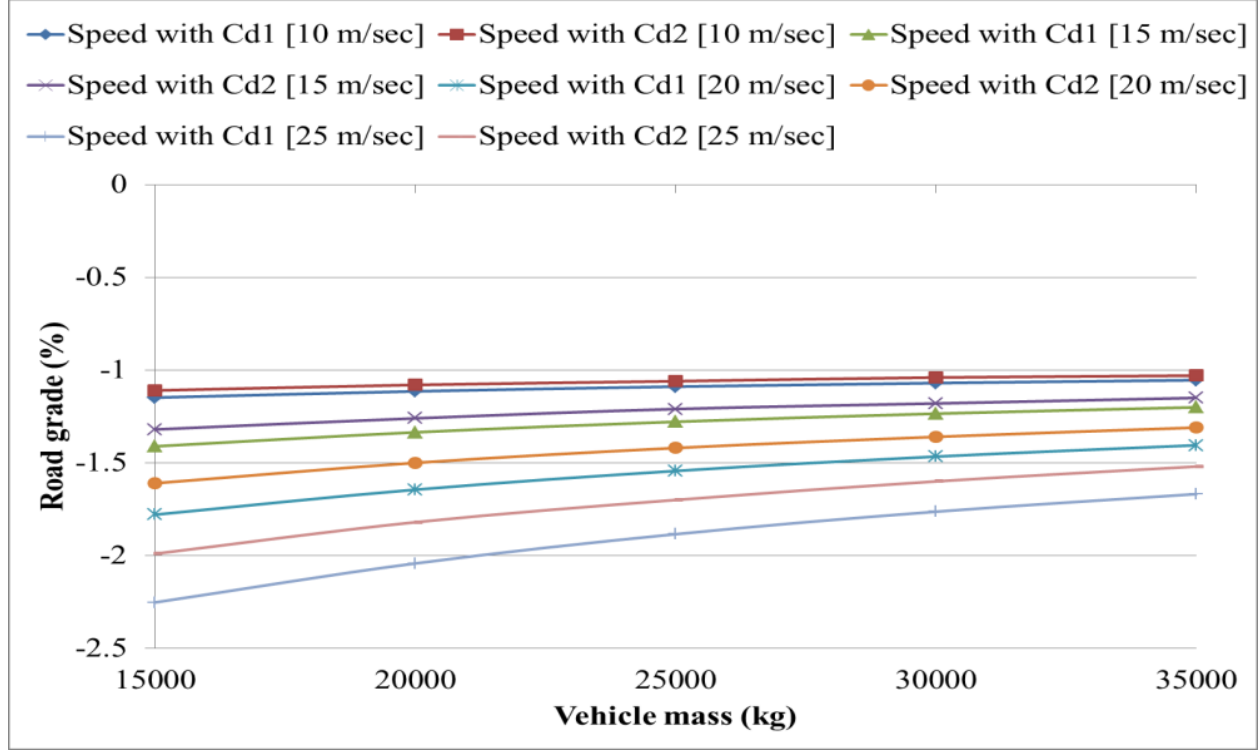

Figure 4.13: Negative road grade when driver needs to start depressing brake with different air drag coefficient values 


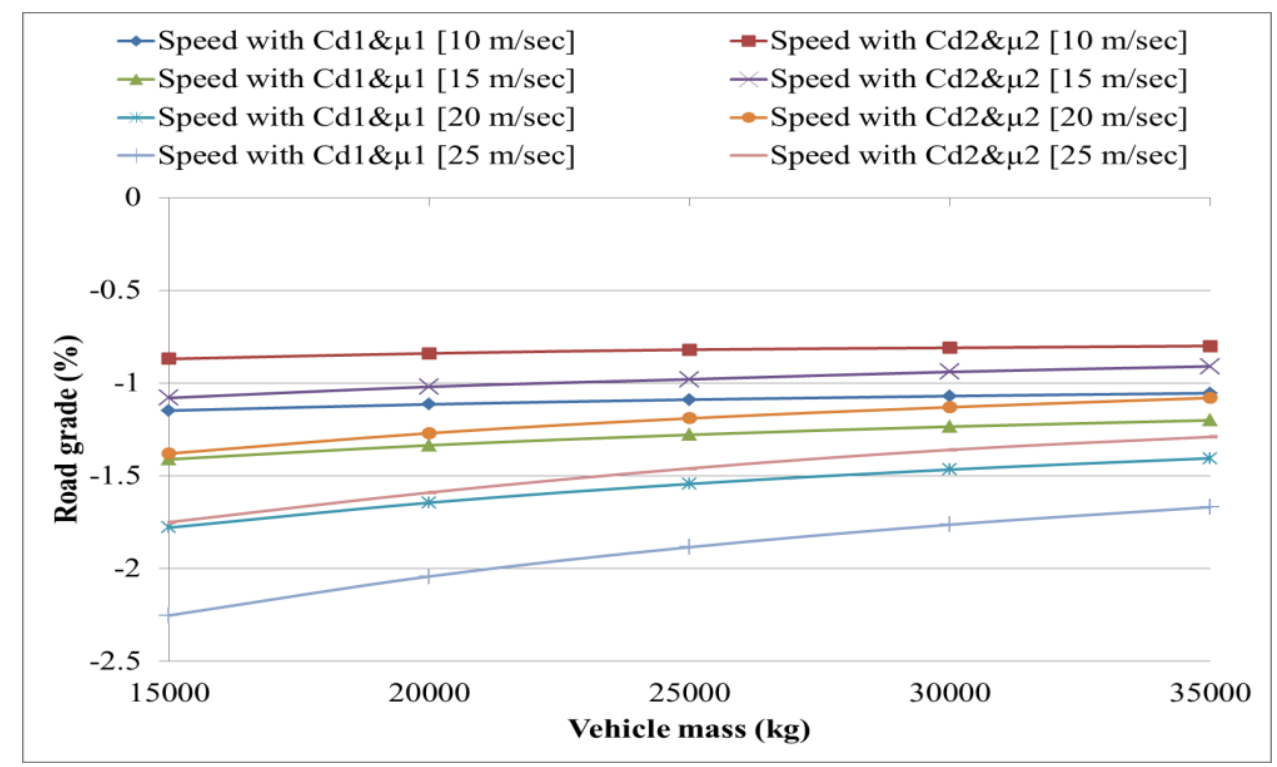

Figure 4.14: Negative road grade when driver needs to start depressing brake with different rolling resistance and air drag coefficients values

The advantage of a hybrid electric vehicle is capturing the free energy while using the brake system. Therefore, when a driver starts depressing the brake earlier descending hills, the free captured energy could be increased. Figure (4.15) shows the negative road grade change (when a driver starts using the brake) due to improved rolling resistance or air drag coefficients. Negative change here has a positive impact on increasing the chance for a driver using the brake earlier and capturing more energy. For example, if the rolling resistance and air drag coefficients improved by $25 \%$ for a heavy duty vehicle that is need to use the brake system at road grade less than $-2 \%$, then the driver will need to use the brake system at road grade below $-1.6 \%$. In other words, improving load coefficients (i.e. $\mu$ and Cd) lead to increase trucks' sensitivity for negative road grade. 


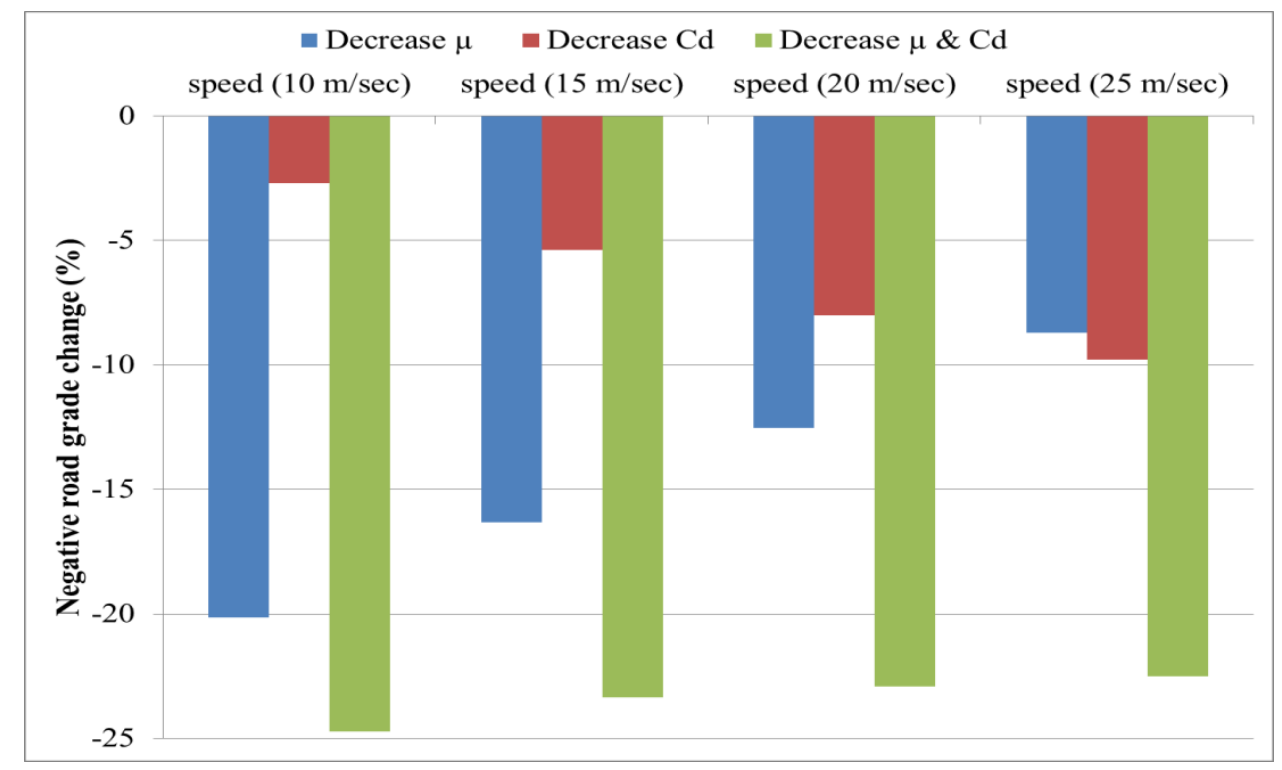

Figure 4.15: Negative road grade change due to improved rolling resistance and air drag coefficients.

As a consequence of improving the trucks' sensitivity to negative road grade, the expected free energy to capture using the brake system will increase. Figure (4.16) shows the impact of improving rolling resistance and air drag coefficients on total power available descending hills. Negative power demand can increase up to about 35\% as shown in Figure (4.17). This potential increase in power demand obtainable descending hills is promising about hybrid electric vehicle future. These calculations' results gave a good reason to do this study and to propose algorithms to improve ESS management in opportunistic regeneration. This conclusion leads to an important question: which is worth more, improving truck design, such as decrease frontal area and improving load loss coefficients, or improving a HEV performance? Any improvement in vehicle load losses can contribute in boosting HEV benefits in perspective of fuel economy improvement. If the vehicle load losses decrease, then the vehicle sensitivity for road grade and deceleration to use the brake system increase. In other words, heavy duty vehicle weight will not change due to coefficients (i.e. air drag and rolling resistance) improvement, so the force (using the brake system) needed to compromise the vehicle speed will increase. 


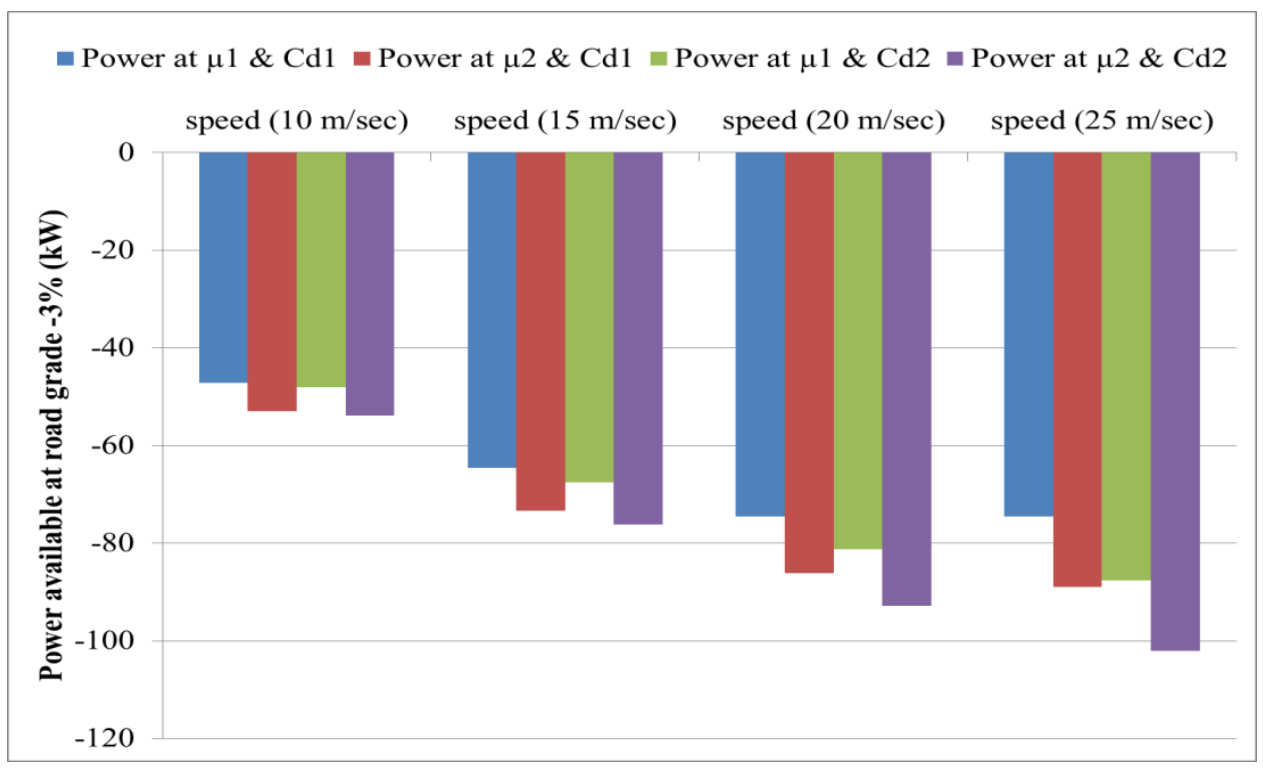

Figure 4.16: Power available to capture descending hill (-3\%) for heavy duty vehicle $(25,000 \mathrm{~kg})$

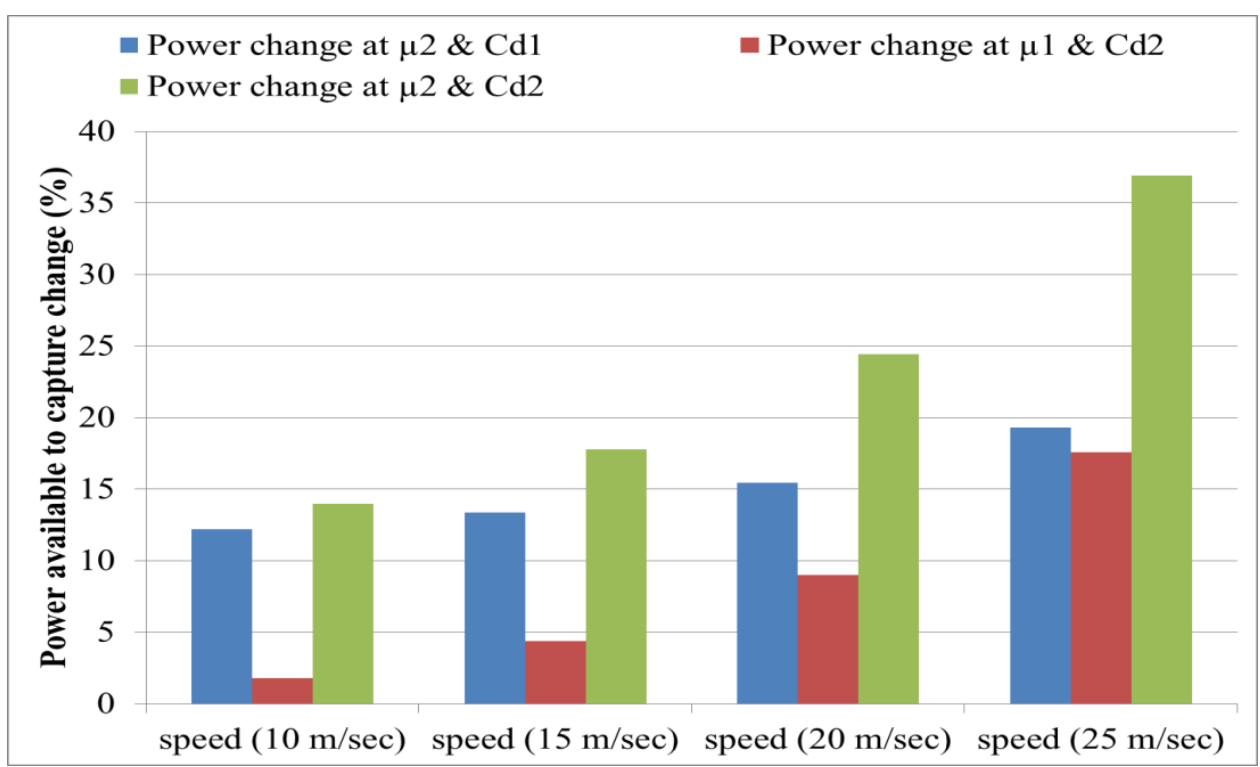

Figure 4.17: Impact of improving the rolling resistance and the air drag coefficients on heavy duty vehicle $(25,000 \mathrm{~kg})$ descending hills $(-3 \%)$. 


\subsection{Evaluate the results goodness}

The main objective of modifying vehicle propulsion for parallel heavy duty hybrid electric vehicle is to improve fuel economy and enable the powertrain system to maintain vehicle speed (adequate power) during all activities. In other words, the purpose of this study is to optimize $\mathrm{HEV}$ in perspective of improving fuel economy while maintaining adequate power at the same time. The criteria to evaluate the goodness of the modified model performance are the square of correlation $\left(\mathrm{R}^{2}\right)$ and root mean square error (RMSE) as shown in equation 4.5 and 4.6 [71]. Relative error (RE) is used also to evaluate the goodness of the average simulation speed results compared to the average target speed. The RE equation is defined in equation 4.7.

$$
\begin{gathered}
R^{2}=\left(\frac{\sum_{i=1}^{N}\left(y_{i}-\bar{y}\right)\left(y_{i_{i}}-\overline{y^{\sim}}\right)}{(N-1) s_{y} s_{y^{\sim}}}\right)^{2} \\
R M S E=\sqrt{\frac{1}{N} \sum_{i=1}^{N}\left(y_{i}^{\sim}-y_{i}\right)^{2}} \\
R E=\frac{\left(\bar{y}-\overline{y^{\sim}}\right)}{\bar{y}} * 100 \%
\end{gathered}
$$$$
\text { Equation } 4.5
$$$$
\text { Equation } 4.6
$$

where $y_{i}$ and $y_{i}^{\sim}$ are measured and simulated vehicle speed data respectively, $\bar{y}$ and $\overline{y^{\sim}}$ are their average, $s_{y}$ and $s_{y} \sim$ are their chassis deviation respectively, and $\mathrm{N}$ is the number of data points.

\subsection{Summary}

This section provides the methodology to implement the suggested modification on the vehicle propulsion controller for the direct parallel HEV. This section represents the core of this study. Simple and sophisticated algorithms have been presented in this section. Different scenarios were discussed in this study to explore the impact of information details and precision on modified VPC response. Time for charging or discharging has been estimated based on equipment sizes and efficiencies. The role that the VPC must consider is to explore when drivers are going to use the brake system. Road load equation was used to calculate the negative road grade when drivers start using the brake system. Both ESSs' and electric motor efficiencies' impact on HEV performance have been discussed. 


\section{Chapter 5 : Results and discussions}

\subsection{Model sensitivity for different terrain types}

Looking-ahead control strategy for heavy duty parallel HEV can contribute significantly in

preparing the system for forthcoming traffic conditions regarding vehicle speed and road elevation. The best way to show the impact of looking-ahead strategy on the powertrain system is by using simple terrains and seeing how the vehicle propulsion controller (VPC) will respond. The simple terrains suggested here are the up and down hills at constant road grades with constant vehicle speed.

\subsubsection{Simple looking-ahead VPC control strategy}

The simple terrain types, which have been chosen, are the uphill, downhill and down-uphill terrain types. The VPC controlling strategy with simple looking-ahead algorithm works by opening a time window ahead, for 1 minute period, and detects the traffic conditions regarding vehicle speed and road elevation ahead. The road load equation is applied to estimate if the powertrain is going to need hybridization system for assisting or depositing energy.

Figures (5.1-5.2) show the FC, battery SOC, engine energy losses and electric motor energy losses behavior with and without forthcoming traffic information for descending and ascending terrains, and this was for a direct parallel HEV model $(150 \mathrm{~kW}$ and $10 \mathrm{kWh}$ for electric motor and battery sizes respectively) at $50 \%$ full load $(26,000 \mathrm{~kg})$. When the truck was about to descend a hill, the looking-ahead VPC managed battery SOC in opportunistic regeneration to capture free energy as much as possible. For Figure (5.1b), the VPC worked on sustaining the energy that was captured during descending hills to use later if needed. In other words, the proposed strategy works by switching sustaining and depleting battery SOC during the different vehicle activities. Moreover, the response of looking-ahead VPC was obvious when the truck was ascending a hill or descending it. When the truck was about to go uphill, the looking-ahead VPC managed battery SOC in opportunistic regeneration as shown in Figure (5.2b). The battery SOC could be managed incorporates engine charging when no braking opportunity exists. The VPC stopped looking-ahead about 10 minutes before the trip ended, so the VPC worked on maintain battery SOC in the middle. The reason to stop looking-ahead before the trip ending is to 
guarantee that the battery SOC will be the same at the end of trip for looking-ahead and without looking-ahead strategies. These results were for scenario two, which is looking at forthcoming speed limit (60 mph for these results) and road grade.

There are several other factors that can show the impact of ITS on the HEV. Fuel consumption change due to modifying the vehicle propulsion controller strategy based on the forthcoming traffic information is one of the important factors that can give a clear idea about the impact of looking-ahead strategy. Figures (5.1b and 5.2b) show the impact of ITS on the integrated fuel consumption for descending and ascending terrains and highway driving cycles. For example, the cumulative fuel consumption was decreased from $23.159 \mathrm{~kg}$ to $22.703 \mathrm{~kg}$, which represents about $-2 \%$ changes in the total fuel consumption when descended the hill. This decreasing in the total fuel consumption came from managing the ESS in opportunistic generation and assures more capacity to capture more free energy when descended a hill. However, for the parallel HEV model ascended the hill as shown in Figure (5.2), there was a small penalty due to managing the ESS and prepare more energy to assist the main power source, which is the engine. The total fuel consumption was increased by about $0.5 \%$, and this increasing is due to using the engine to charge the battery preparing for the forthcoming power demand, which is acceded the engine peak power. Moreover, these increasing in the fuel consumption can be avoided if we didn't prepare the ESS for the forthcoming power demand using the engine. In the real world driving cycles, these penalty from using engine to charge the battery can be compromised as shown in the next sections. The main reason for the chance of decreasing using engine to charge the battery is that the real world terrains are not ascending hills only, but they are ascending and descending hills. Therefore, in this algorithm, the VPC working on sustain any free energy captured due to descending hills until there is need for it to ascend next hills.

Figures (5.1c and 5.2c) show the impact of looking-ahead on the energy losses from engine and the electric motor. The energy losses from the electric motor were worse for the both situations (the descending and ascending hills). The reason for increasing in the energy losses of the electric motor was that the more activity for it. However, this increasing in the energy losses considered small in comparison to the decreasing of the energy losses in the engine. For example, in figure (5.1c) the energy losses for the electric motor were increased from 5.6 MJ to 
6.4 MJ. On the other hand, the energy losses from the engine decreased from $645.8 \mathrm{MJ}$ to 635.1 MJ. Therefore, the energy losses for the electric motor increased about $1 \mathrm{MJ}$, but at the same time the energy losses from the engine decreased about $10 \mathrm{MJ}$.
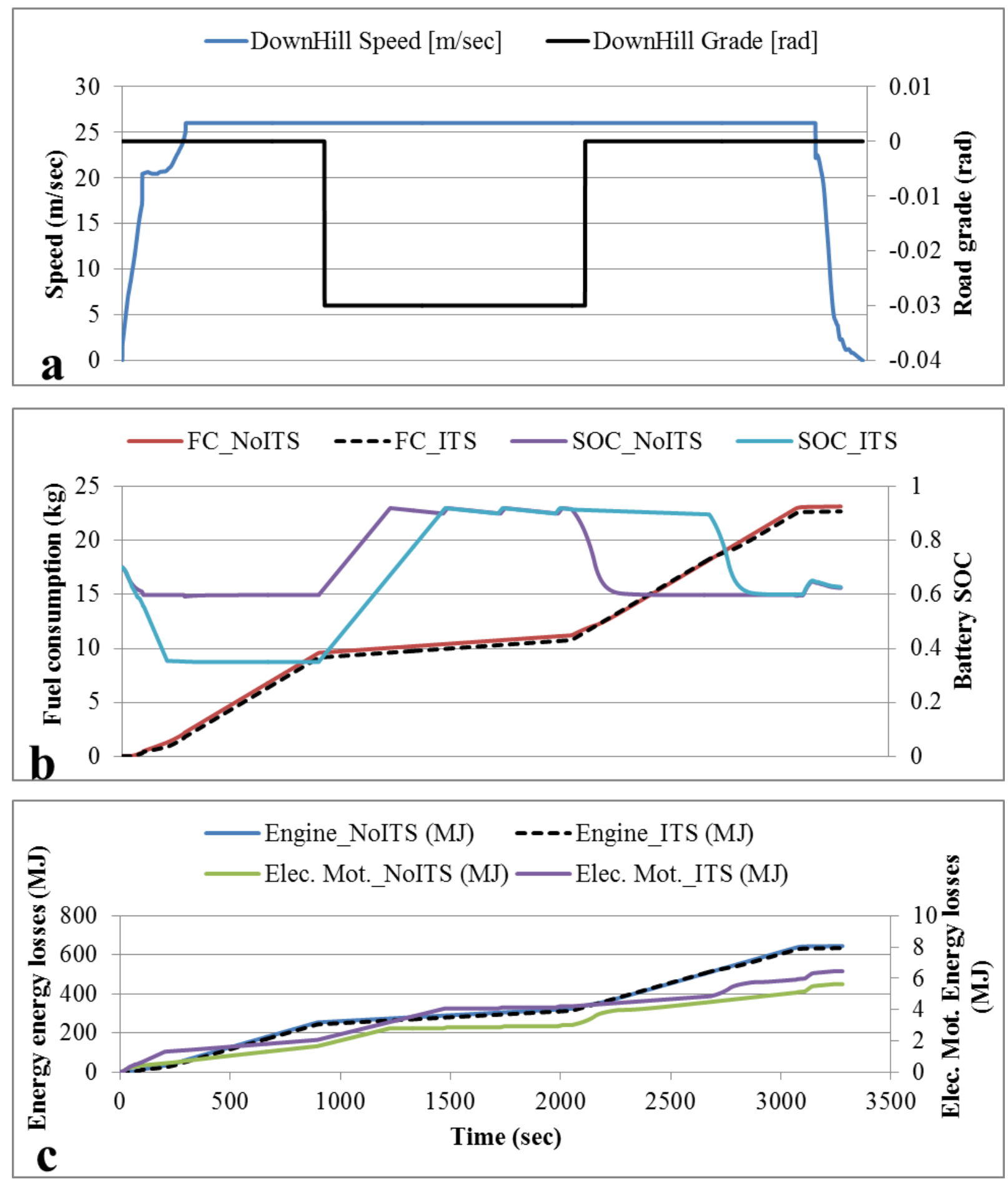

Figure 5.1: Impact of the ITS on FC, battery SOC, engine energy losses and electric motor energy losses for going downhill and highway driving cycle 

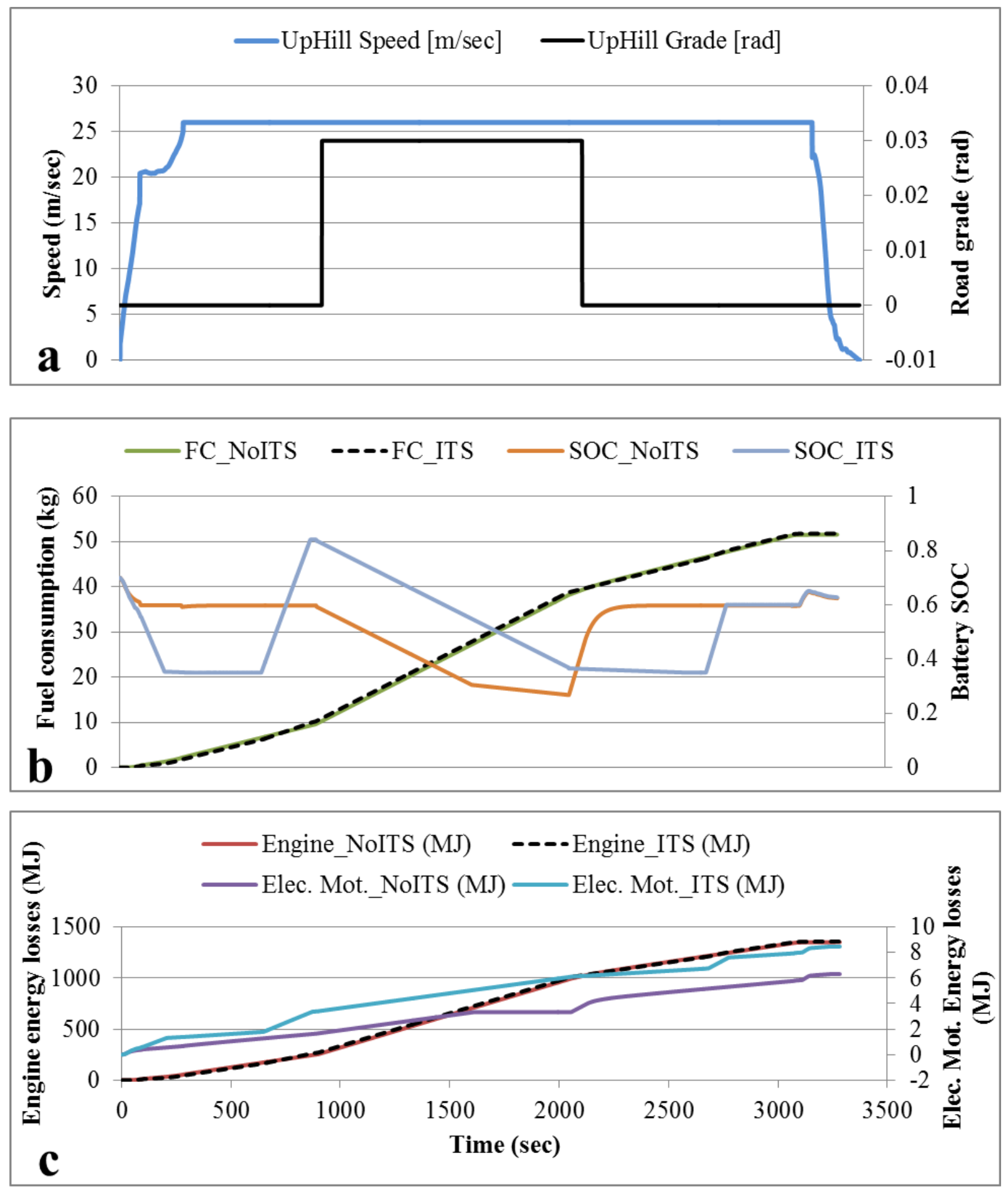

Figure 5.2: Impact of the ITS on FC, battery SOC, engine energy losses and electric motor energy losses for going uphill and highway driving cycle 
Figures (5.3 and 5.4) show the impact of looking-ahead on the ESS management. Different other factors to analyze the impact of looking-ahead were listed previously. The battery SOC changing can give an obvious idea about what is going on and how the VPC respond for the forthcoming power demand. The looking-ahead VPC shows a proper response while the truck was ascending and descending small hills. For ascending and descending small hills, the looking-ahead VPC didn't charge or deplete the battery because there was no need for that (according to road load equation as explained in the methodology section). For example, in Figure (5.4) the VPC didn't charge the ESS to prepare for the $-0.5 \%$ and $-2 \%$ road grades. The reason for that is the road grade until $-2 \%$ will not make the driver to depress the brake pedal to compromise the vehicle speed on highway. It has been calculated and proven that there is a certain negative road grade for every type of activity then the driver needing to depress the brake pedal to compromise the vehicle speed, please check the section 4.6 of chapter four for more details. Moreover, at the road grade $0.5 \%$ and $2 \%$ the VPC didn't charge the battery because the total forthcoming power demand didn't accede the peak engine power. Appendixes $\mathrm{C}$ shows the impact of looking-ahead strategy on the total forward and reverse energy losses, also the total equipment efficiencies have been shown for the highway and uphill driving cycle.

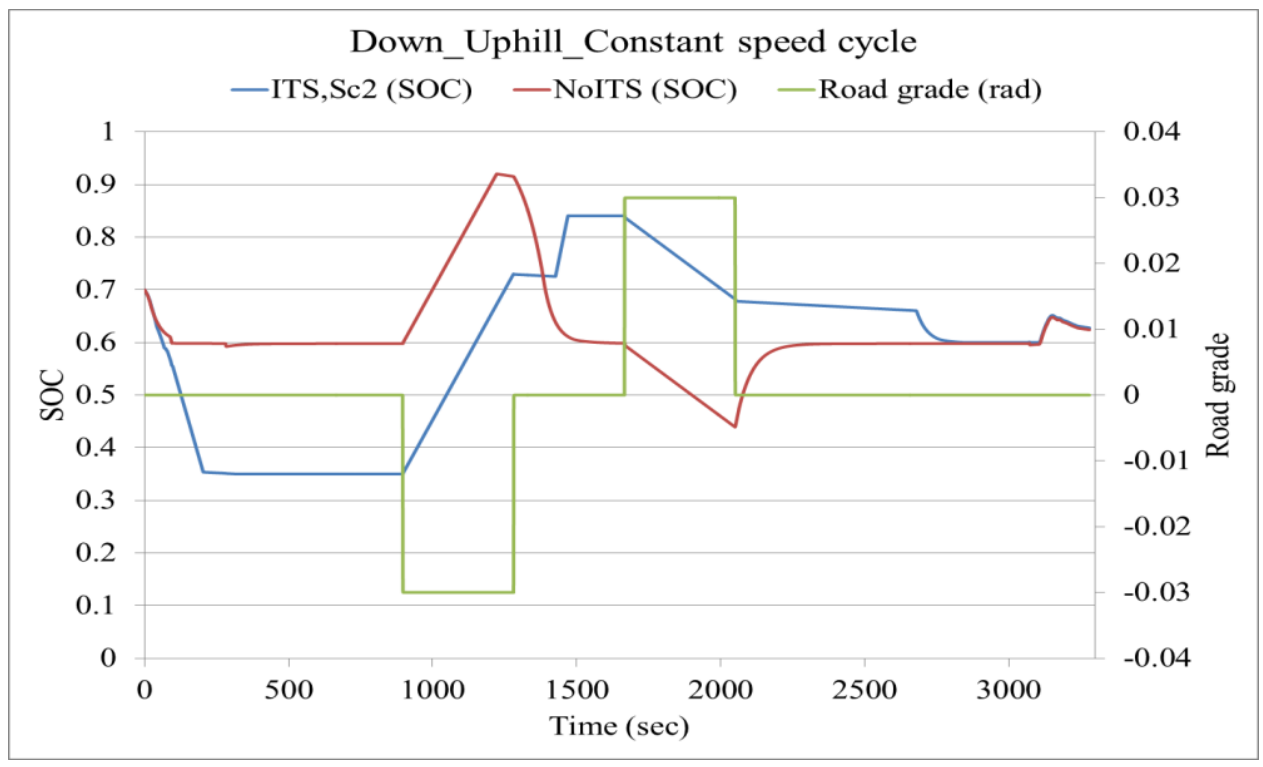

Figure 5.3: Impact of looking-ahead on the battery SOC for descending and ascending hills and highway driving cycles 


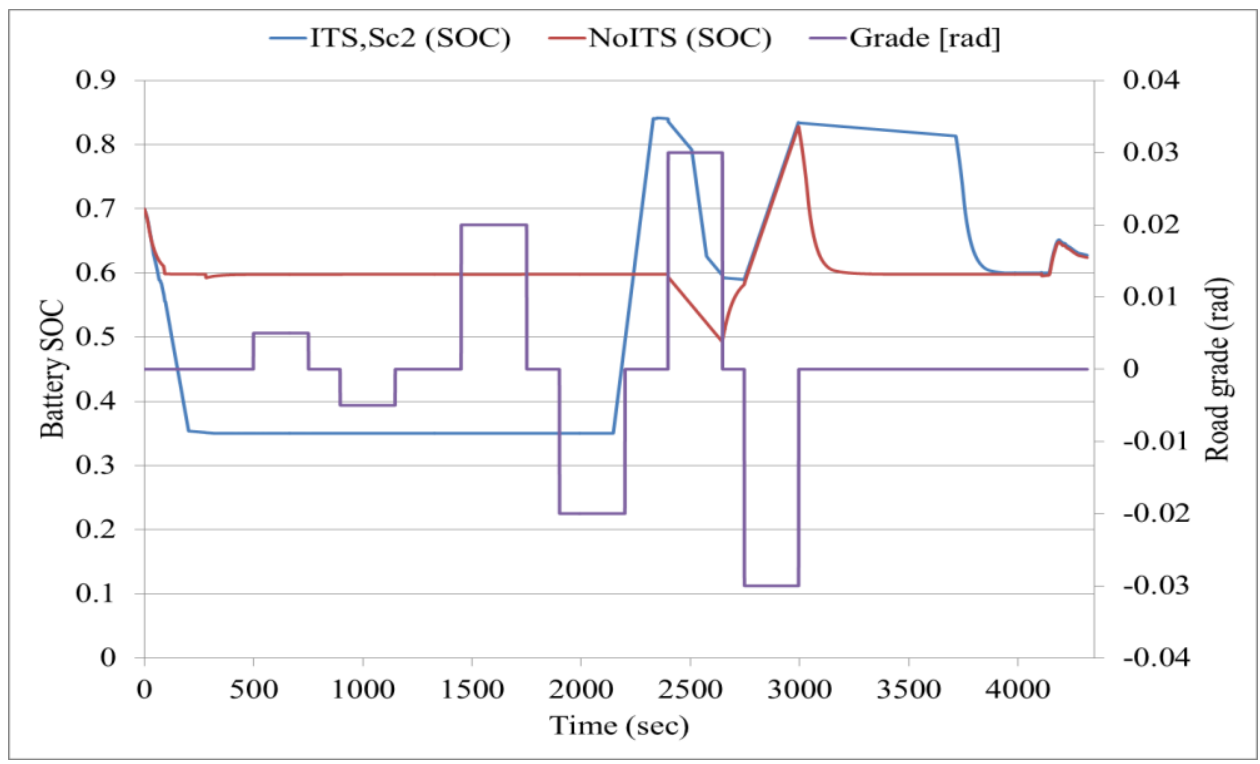

Figure 5.4: Impact of looking-ahead on the battery SOC for different updownhill grades and highway driving cycles

\subsubsection{Sophisticated looking-ahead VPC control strategy}

The battery SOC management may represent an important reflection on the proposed lookingahead strategy effectiveness. Figure (5.5) reveals the VPC managing for the energy storage system with and without looking-ahead strategy (ITS) for a $150 \mathrm{~kW}$ and $10 \mathrm{kWh}$ electric motor and battery pack energy, respectively. Figure (5.5) presents the looking-ahead VPC management for the battery SOC in advance to prepare the system for forthcoming power demand. It can be noticed that at the beginning of the trip the VPC depleted the battery SOC because the electric mode at low speed is better than conventional mode [35]. The VPC didn't charge or discharge the ESS when the vehicle was about to ascending or descending small hills, because there was no expected energy to capture or additional power demand. However, when the vehicle was ascending the hill with $3 \%$ road grade for relatively short time, the VPC charged the ESS with same expected additional amount of power demand using the engine. Whereas the VPC without looking-ahead strategy kept the battery SOC at the default threshold (60\%), there was not enough alternative power during ascending the entire hill. These results are for a direct parallel HEV model (150 kW and 10kWh for electric motor and battery sizes respectively) with 50\% full load. The VPC with sophisticated algorithm showed as same respond as for simple algorithm regarding the long ascending and descending hills. 


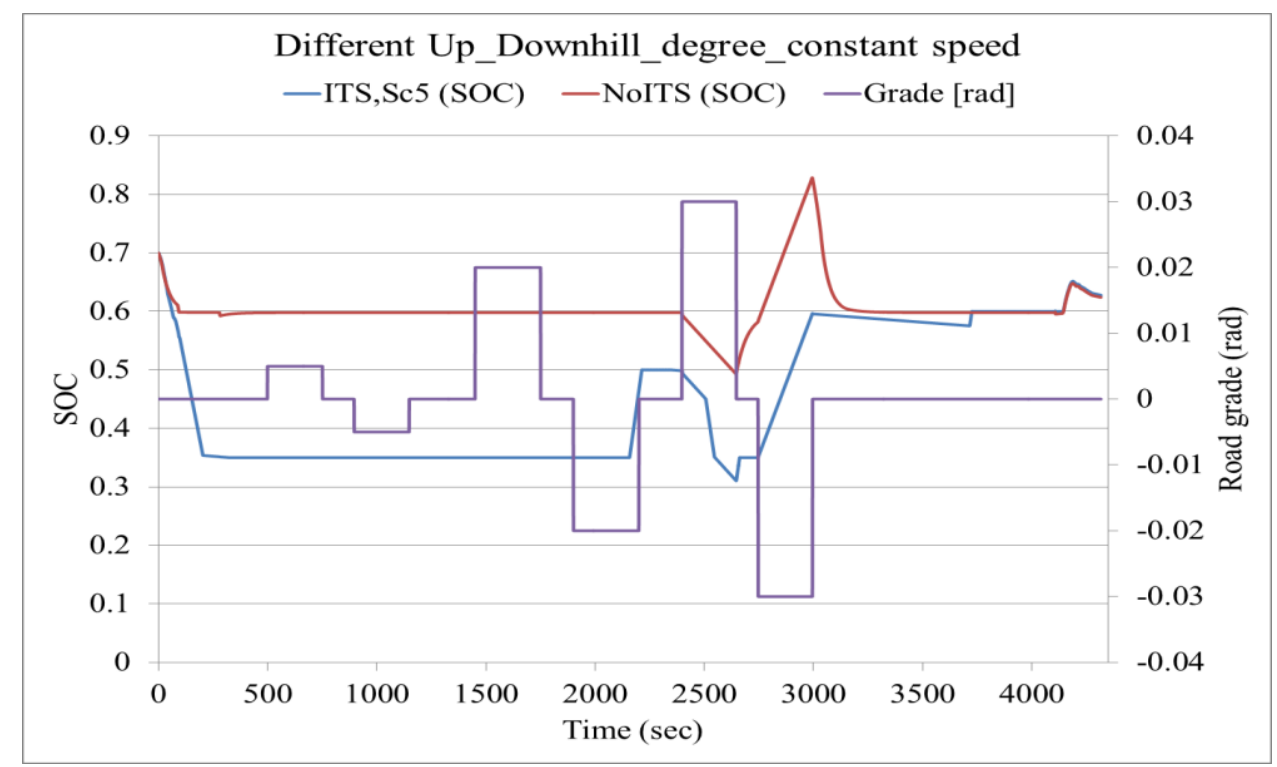

Figure 5.5: Sophisticated algorithm for different up-downhill grades and highway driving cycle

Figure (5.6) shows the response of the VPC regarding battery SOC with simple and with sophisticated algorithm. It is obvious that the sophisticated algorithm can work better especially, for small ascending or descending hill terrain type. The sophisticated algorithm can save more fuel by avoiding using engine to charge the battery unless there was a real need for that. Figures for simple and advance algorithms on uphill, downhill, and down-uphill terrain didn't show difference in battery SOC response. The reason for that is due to the long-slope road. For Figure (5.6) the battery SOC is showing how the model sophistication may impact the energy demanded from the engine to charge the battery. It is well known that it's better to avoid charging the battery using engine and to avoid the energy losses due to electric motor and battery equipment (see the trip period 2000-3000 seconds for Figure (5.6)). Figure (5.7) shows the cumulative engine energy losses during the test. The Energy losses change during the test can give a good understanding for the respond of the VPC regarding the forthcoming power demand. 


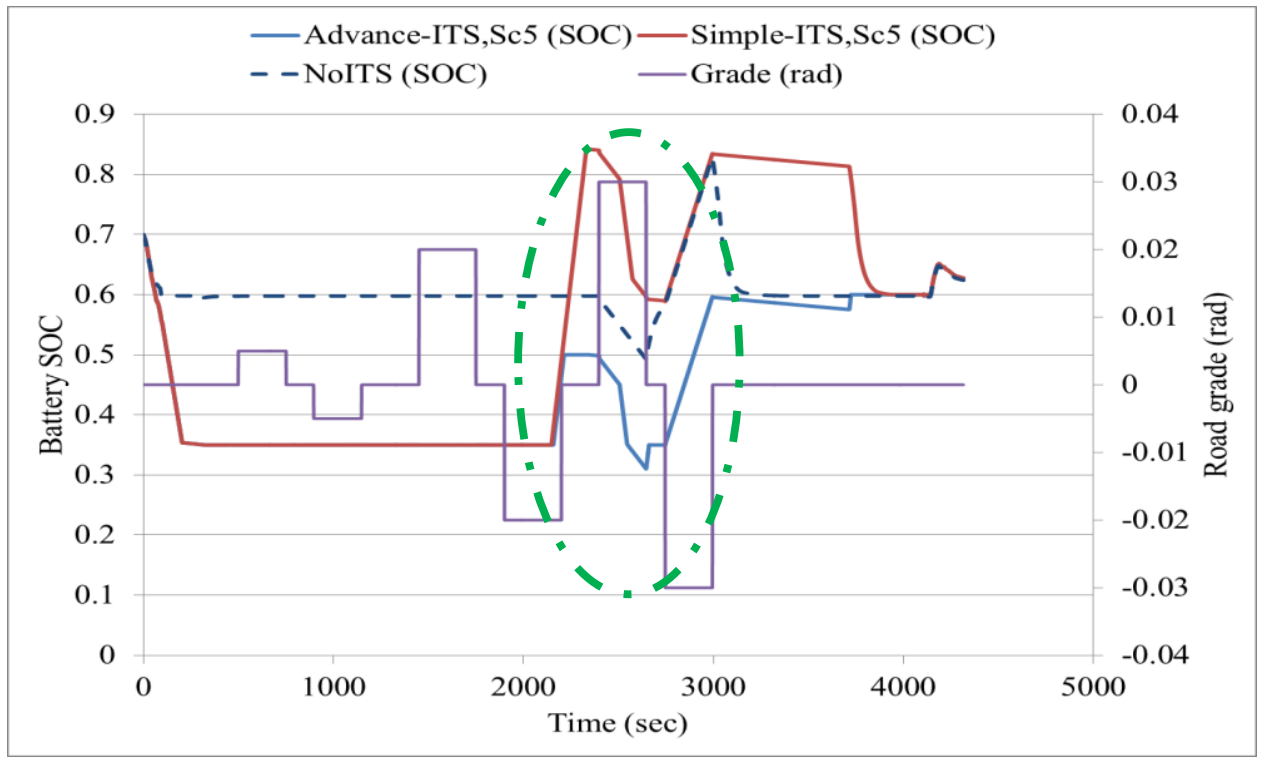

Figure 5.6: Battery SOC variation on different road grade degrees with simple and sophisticated looking-ahead propulsion controller.

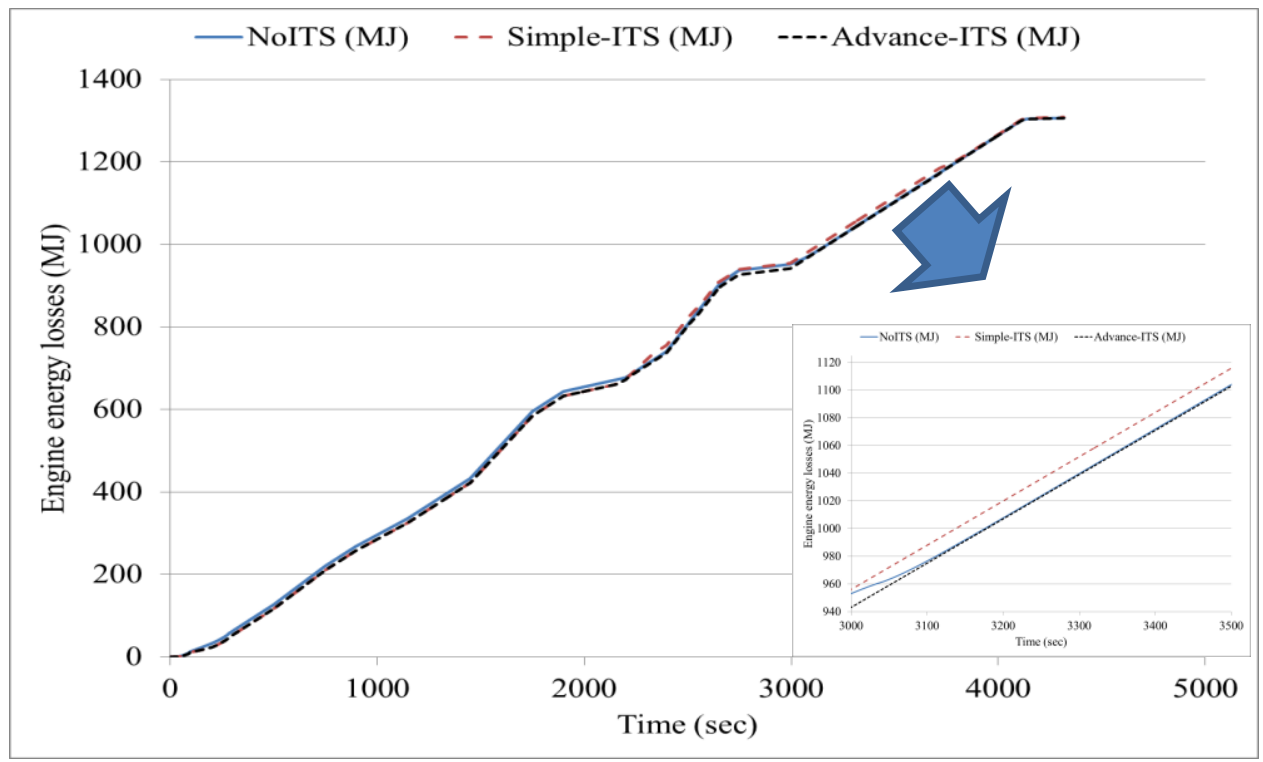

Figure 5.7: Engine energy losses with No ITS, simple ITS, and advanced

ITS control strategy for the different road grade degrees with highway driving cycle 
Figures (5.8 and 5.9) show the impact of the looking-ahead strategy on accumulated fuel consumption for different up-downhill terrains. Accumulated fuel consumption can reflect the impact of looking ahead on the engine. Moreover, it can help analyze the looking-ahead control strategy impact on the main power source, which is the engine, due to ascending and descending hills. In other words, each trip can be divided to sectors and investigated the impact of lookingahead on each sector. It is obvious that the VPC demands more power from the engine with looking-ahead (when ascending hills) to prepare energy for upcoming event. So it's logical to expect that the FE for the HEV ascending hills only will not improve, but it may be decreased due to using the engine to charge the ESS. As explained before all charging and discharging events for the ESS have a penalty which is equipment efficiency losses, as explained in details before. The accumulate fuel consumption shown in Figure (5.8) were $51.541 \mathrm{~kg}$ without lookingahead and $51.784 \mathrm{~kg}$ with looking-ahead control strategy, which means the accumulated FC increased by $0.5 \%$ due to looking-ahead strategy. Moreover, the accumulated FC shown in Figure (5.9) were $36.574 \mathrm{~kg}$ without looking-ahead and $36.412 \mathrm{~kg}$ with looking-ahead control strategy, which means the accumulated FC decreased by $0.5 \%$ due to the looking-ahead strategy.

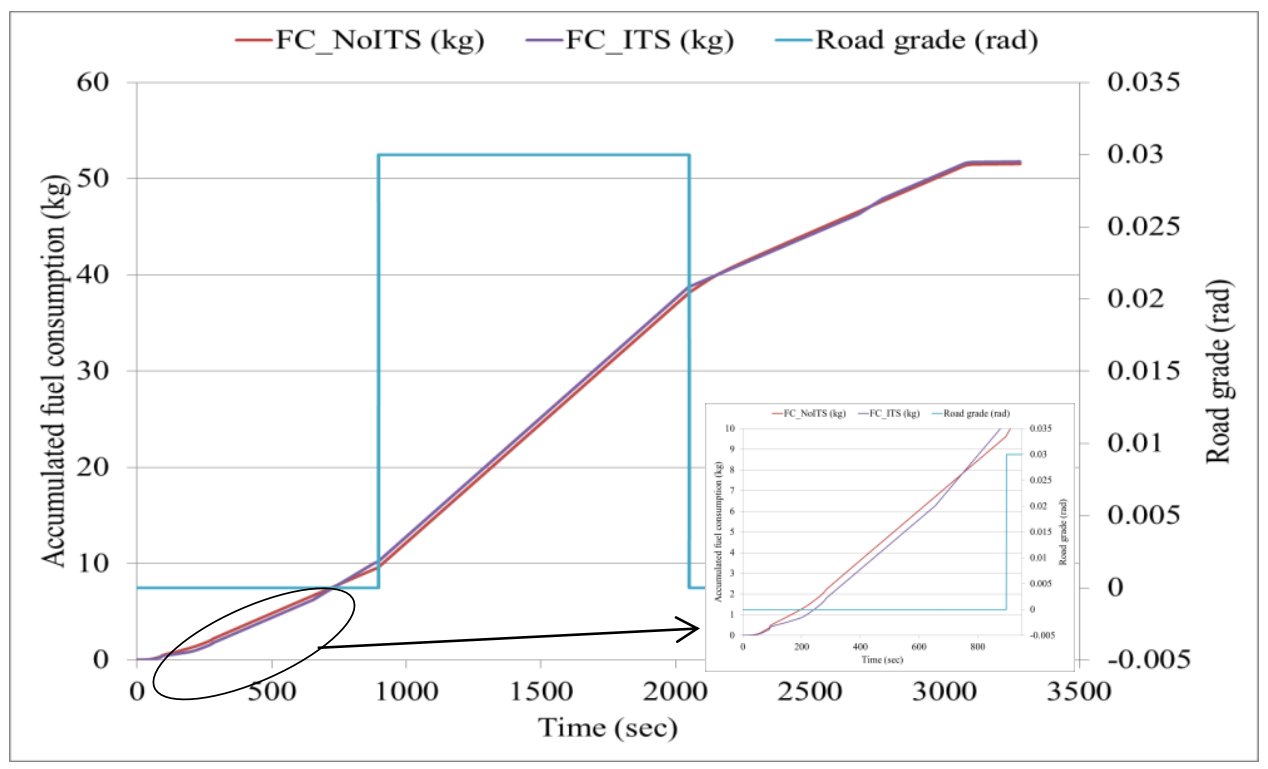

Figure 5.8: FC on uphill highway driving cycle for parallel HEV (150 $\left.\mathrm{kW} \_10 \mathrm{kWh}\right)$ at $50 \%$ load 


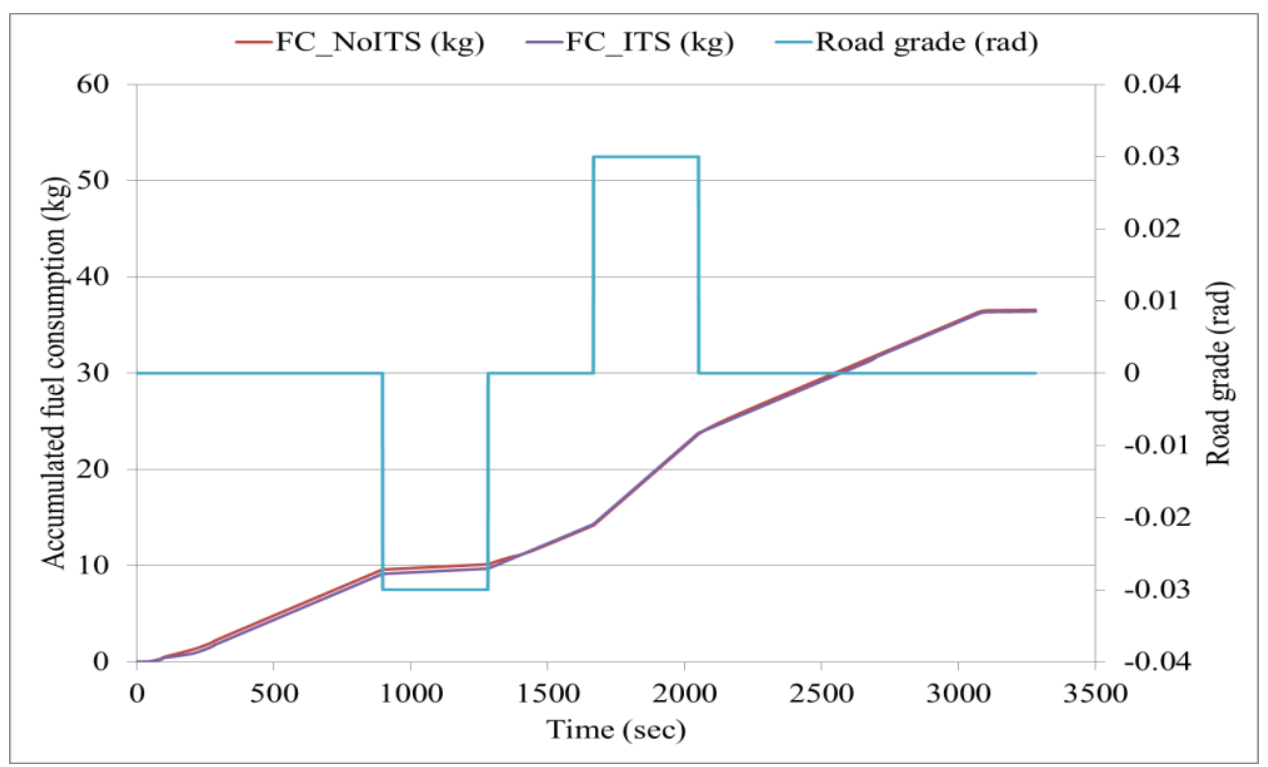

Figure 5.9: FC rate on down-uphill highway driving cycle for parallel HEV (150 kW_10kWh) at $50 \%$ load

\subsection{The impact of looking-ahead control strategy for parallel HEV on fuel economy and powertrain management}

\subsubsection{Ascending and descending hill constant speed driving cycles}

Since a hybrid electric vehicle fuel economy improvement depends mainly on capturing free energy using the brake system, the looking-ahead control strategy may play a role to optimize fuel economy and total powertrain's power (i.e. engine and electric motor). The fuel economy has been calculated for different terrain types. The fuel economy represents one of the most important factors to evaluate the worth of any powertrain improvement. However, there are several other factors designers might need to consider. For example, the alternative energy system effectiveness and maintaining adequate power represent other factors to consider. Figure (5.10) shows the fuel economy for the truck model with different hybridization equipment sizes at different up-downhill terrains and $50 \%$ full load $(26,000 \mathrm{~kg})$ with no ITS and ITS respectively in comparison for the conventional truck. In Figure (5.10) the term Conv_uphill stands for conventional vehicle model with ascending hill, and Uphill-NoITS or Uphill-ITS mean parallel HEV with and without looking-ahead strategy respectively. 


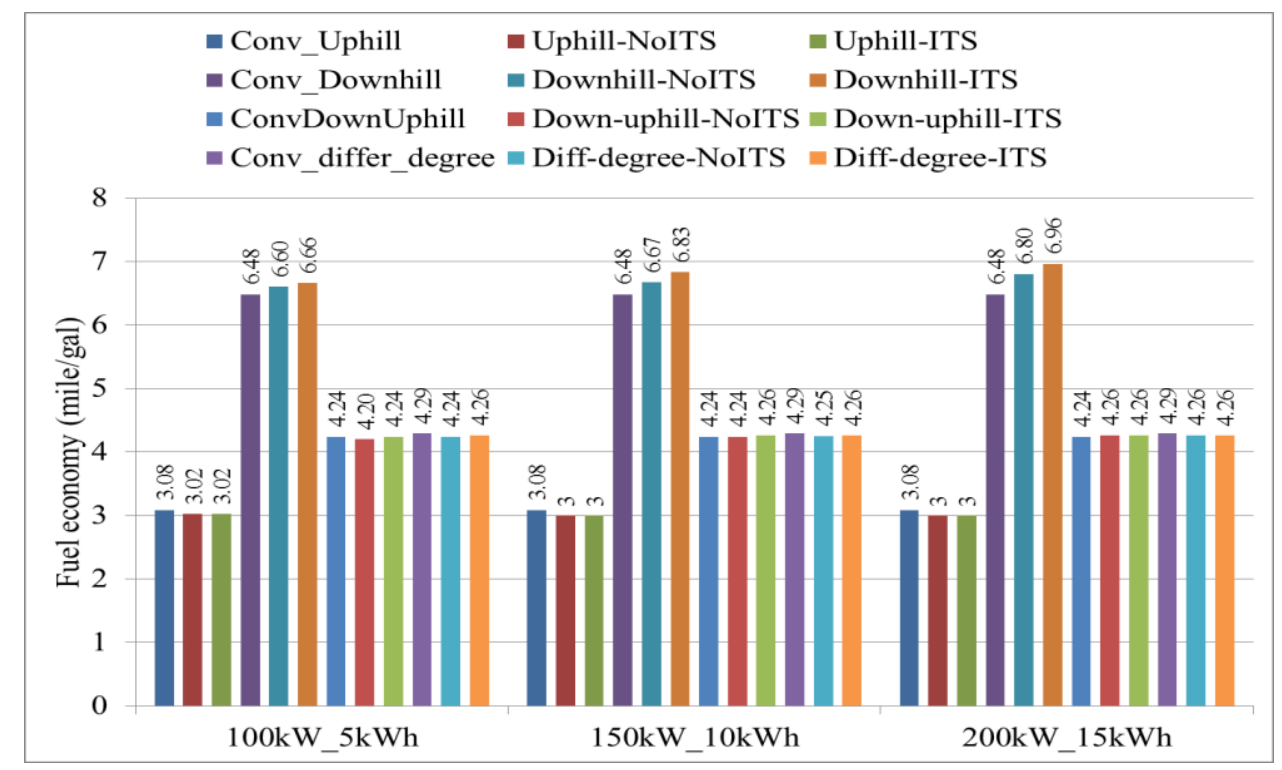

Figure 5.10: Fuel economy for different up-downhill terrains for conventional model and parallel HEV with different equipment sizes with $50 \%$ load.

Fuel economy may be increasing due to the modification of vehicle propulsion controller. The modification on the VPC was to enable looking-ahead and know in advance the forthcoming traffic conditions. Figures (5.11-5.14) show the percentage change in fuel economy for different vehicle configurations (i.e. conventional and parallel HEV) at 50\% load with NoITS and ITS, respectively. The equation used to calculate the percentage change is as following:

Percentage change $=\frac{F E_{I T S}-F E_{N o I T S}}{F E_{N o I T S}} * 100$

Equation 5.1

Percentage change $=\frac{F E_{N o I T S}-F E_{c o n v}}{F E_{c o n v}} * 100$

Equation 5.2

Where $\mathrm{FE}_{\mathrm{ITS}}$ is the fuel economy for different vehicle configuration models with looking-ahead strategy, $\mathrm{FE}_{\mathrm{NoITS}}$ is the fuel economy for different vehicle configuration models without lookingahead strategy, and $\mathrm{FE}_{\mathrm{conv}}$ is for conventional vehicle configuration. 
One of the most important factors when implementing a comparison between different vehicle configurations (conventional vehicle, conventional parallel HEV, and looking-ahead parallel HEV models) is the fuel economy improvement.

Figure (5.11) shows the fuel economy change for a parallel HEV (without looking-ahead) and conventional vehicle model at $50 \%$ full load and different terrain types with constant highway speed driving cycles. The results revealed that the fuel economy change was positive on descending hill terrain. However, the fuel economy change for ascending hill was negative, which could be explained because of the extra power needed from the alternative power system (battery pack). Moreover, the parallel HEV model was able to maintain adequate power more than the conventional model while ascending hill.

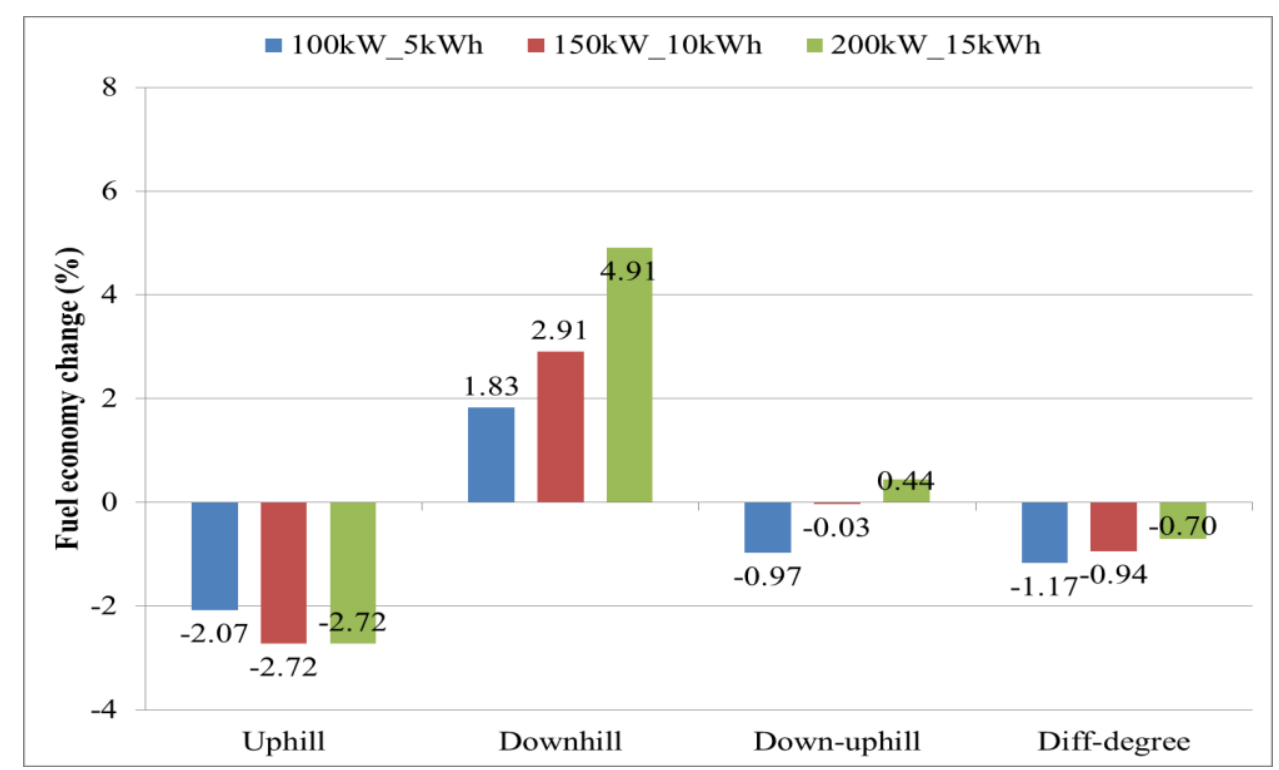

Figure 5.11: Fuel economy change for a conventional and parallel HEV with No ITS control strategy model

Figures (5.12 and 5.13) represent the fuel economy change for parallel HEV with looking-ahead strategy and conventional vehicle model, and parallel HEV with and without looking-ahead respectively. It can be seen from this Figure (5.14) that there is no change in the trend of 
ascending hill terrain between parallel HEV with and without looking-ahead when comparing both of them with conventional vehicle model. On the other hand, in the case of parallel with looking-ahead configuration it has been shown that the alternative energy is doubled, as mentioned in the explanation of the suggested algorithm (more details will be discussed in next sections).

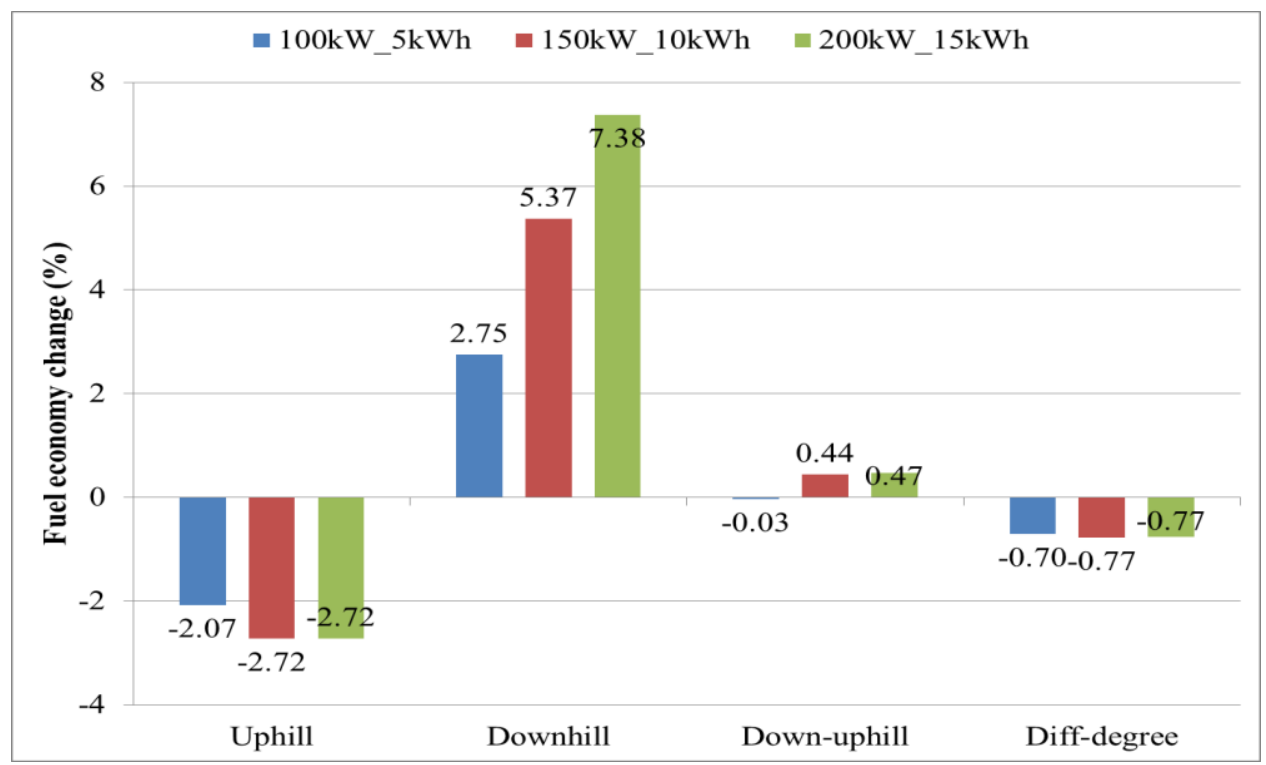

Figure 5.12: Fuel economy changing for a conventional and parallel HEV with ITS control strategy model

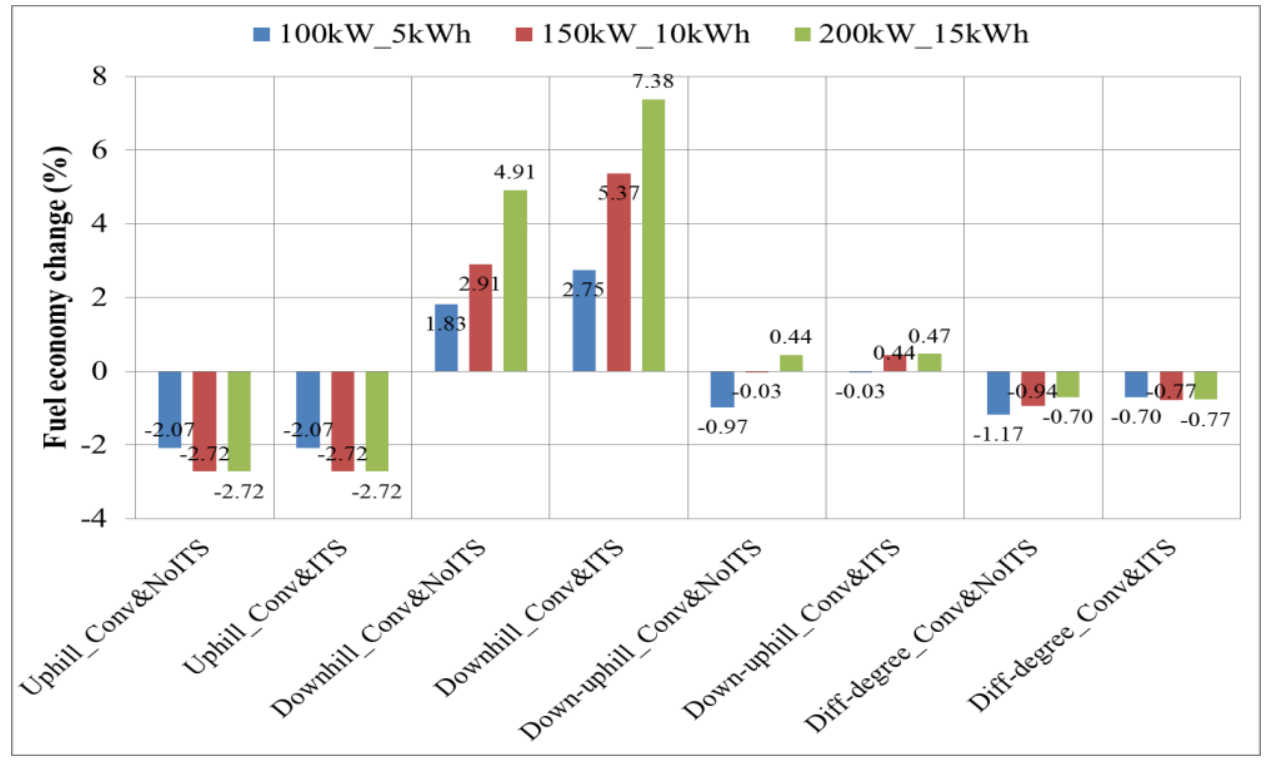

Figure 5.13: Fuel economy changing for a conventional and parallel HEV with ITS once and No ITS second control strategy model 


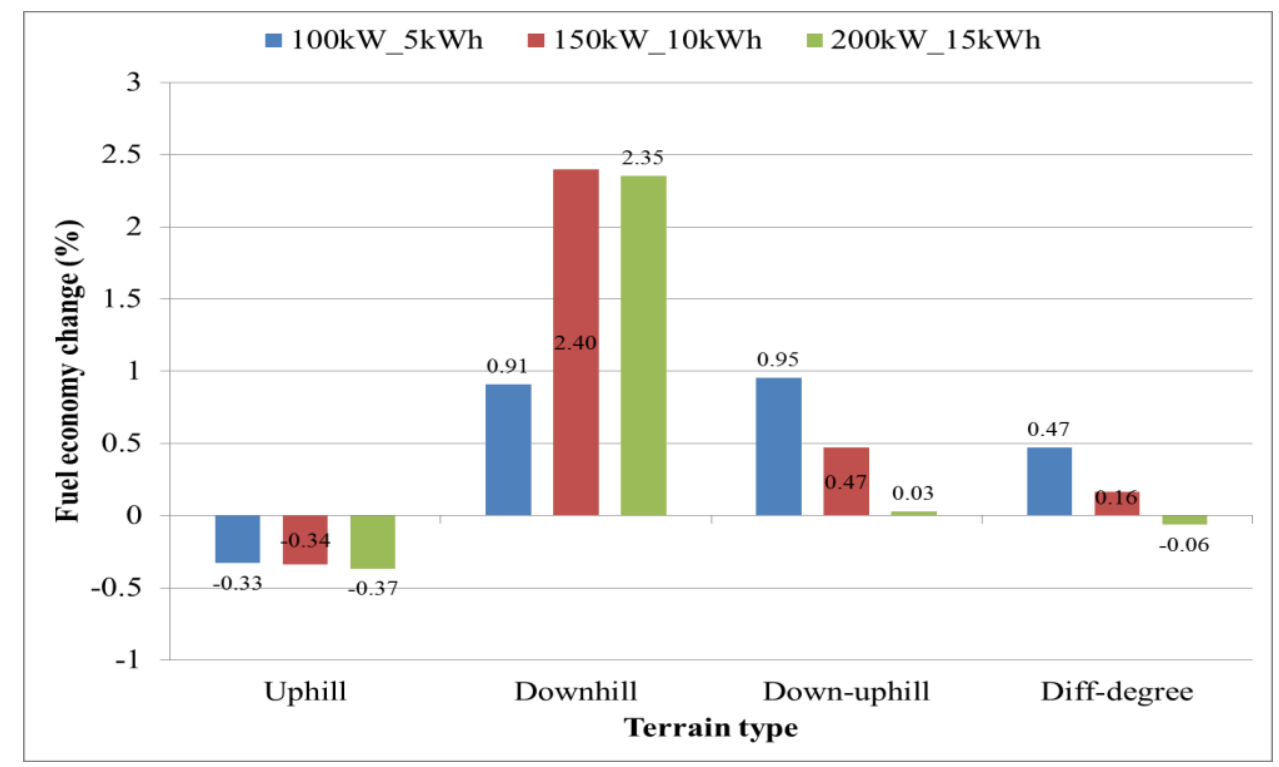

Figure 5.14: Fuel economy change for a parallel HEV with ITS and No

ITS control strategy model

The looking-ahead control strategy could impact the powertrain such as engine efficiency and amount of free energy captured. Figures (5.15 and 5.16) show the fuel hot map for the engine on uphill and constant speed driving cycle. This parallel HEV model was $100 \mathrm{~kW}$ and $5 \mathrm{kWh}$ for electric motor and battery, respectively at $50 \%$ load. These Figures didn't show a significant change in the engine activity. However, with downsized engine power the engine hot map activity changes were obvious as shown later in this Chapter. Moreover, Figure (5.17) shows the impact of looking-ahead on energy captured in comparison to without looking-ahead strategy for different up-downhill terrains. Figure (5.18) shows the regenerative braking recovered at battery change due to looking-ahead strategy in comparison to without looking-ahead strategy for different real world driving cycles. Figures (5.19 and 5.20) show the impact of looking-ahead strategy on the ESS effectiveness for down-uphill constant highway driving cycle. The VPC without looking-ahead maintained the battery SOC in the middle to give an equal chance for charging, when the driver hit the brake pedal, and discharging, when the driver demanded power that surpasses the peak engine power. 


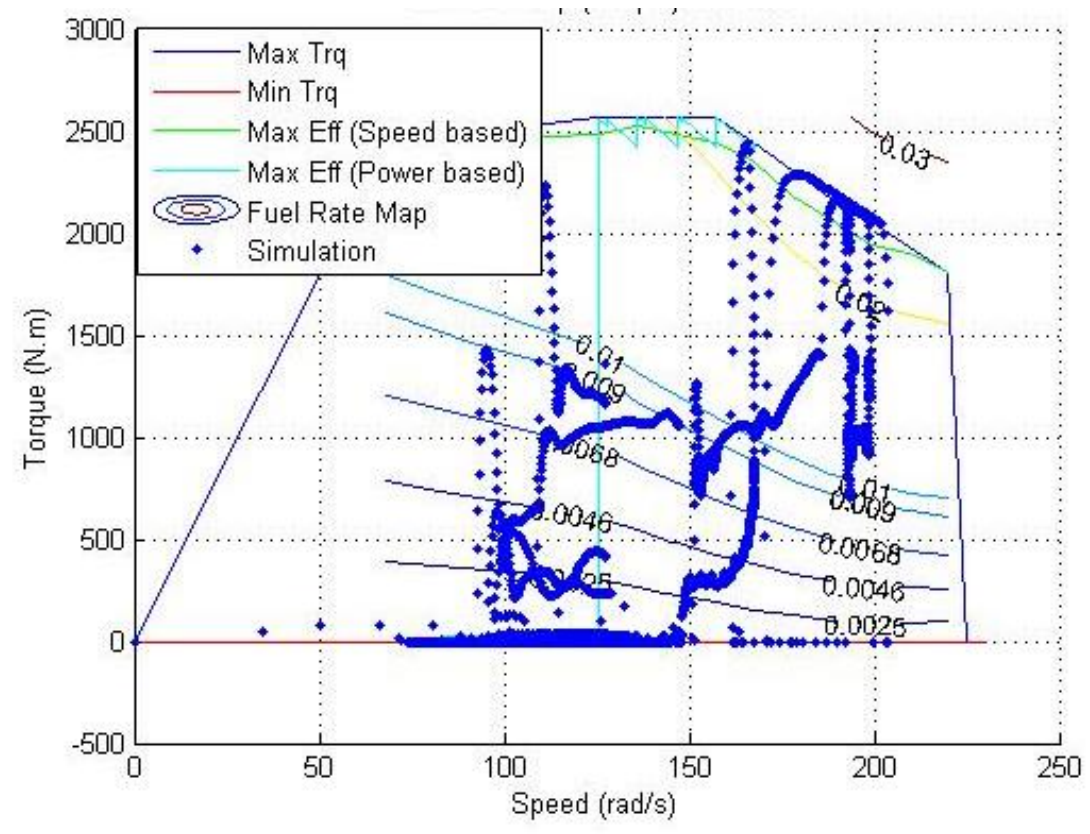

Figure 5.15: Fuel hot map for parallel HEV model (50 \% load) with looking-ahead strategy

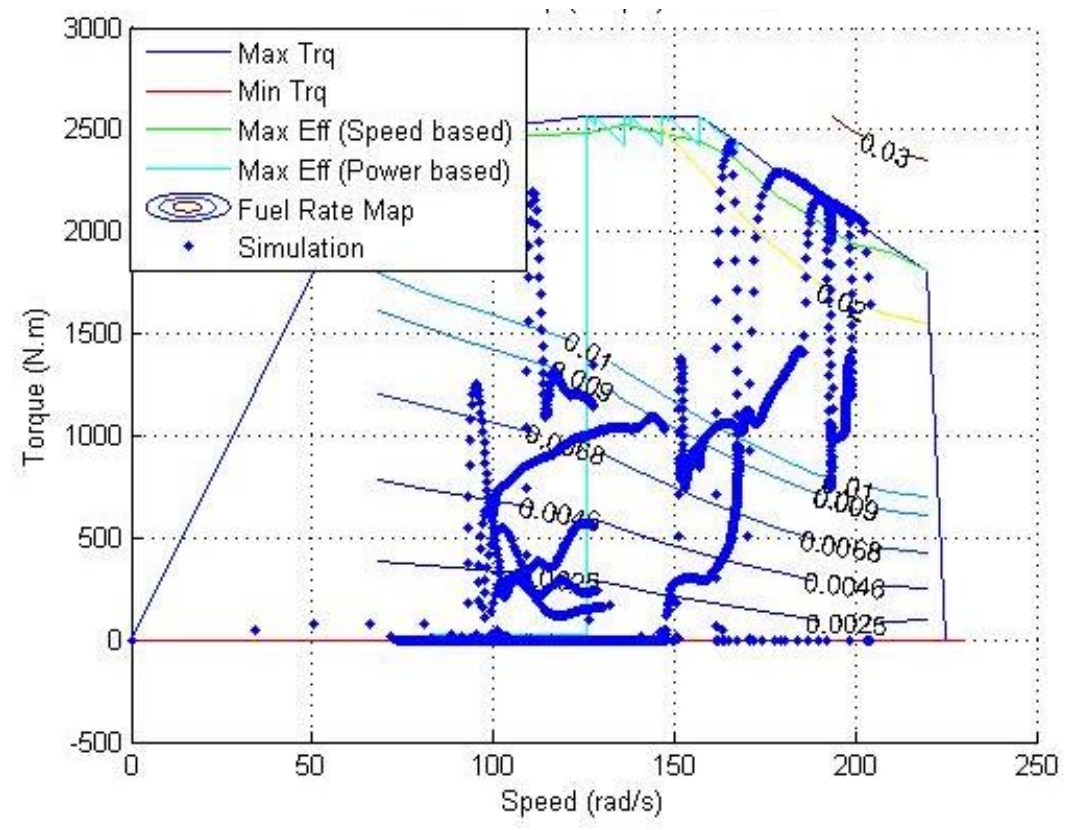

Figure 5.16: Fuel hot map for parallel HEV model (50 \% load) without looking-ahead strategy 


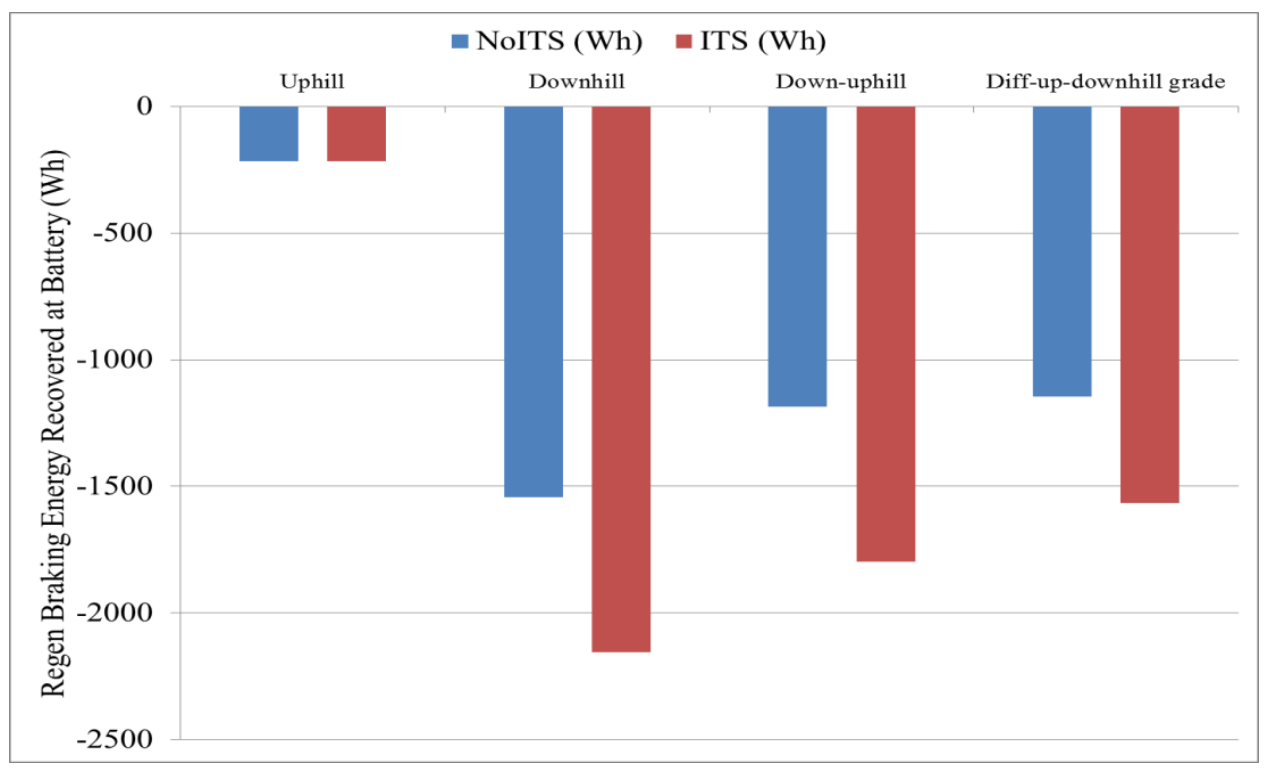

Figure 5.17: Regenerative braking recovered at battery for parallel HEV model (100 kW_5kWh) with $50 \%$ load

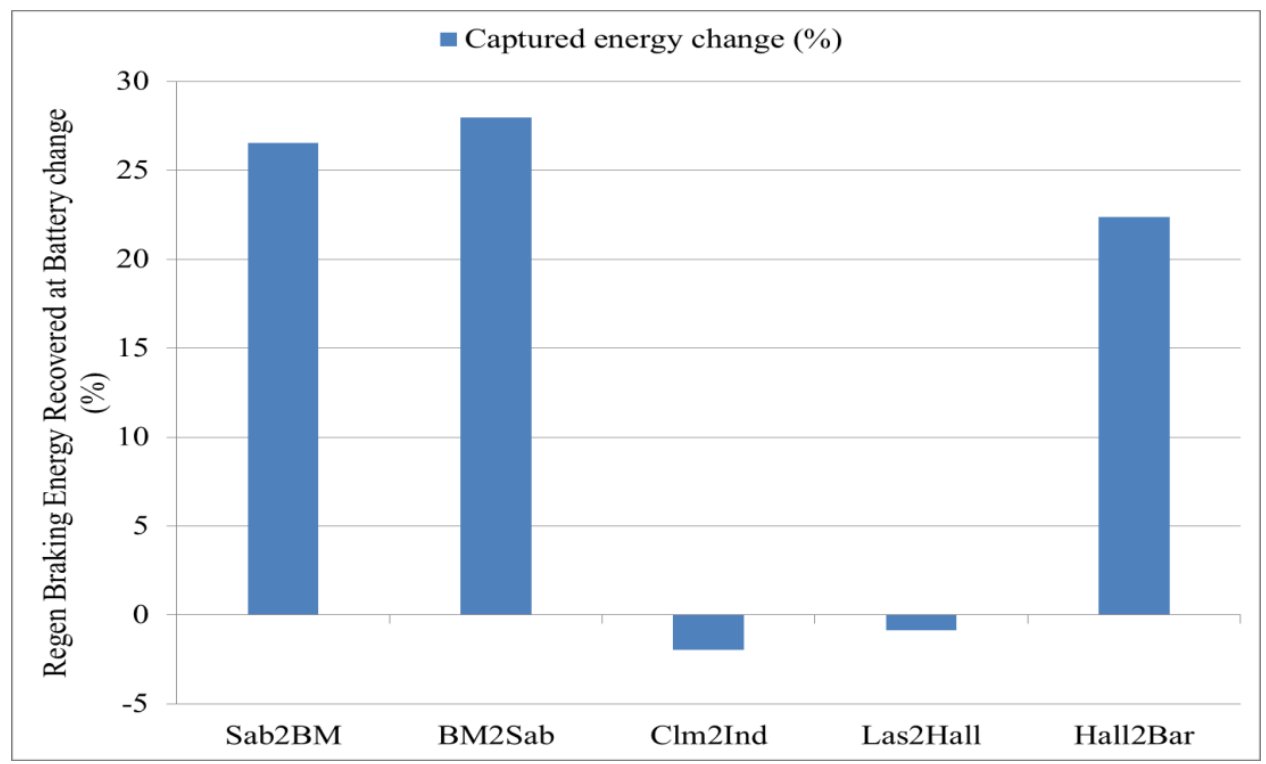

Figure 5.18: Regenerative braking recovered at battery change for parallel HEV model (100 kW_5kWh) with $50 \%$ load 


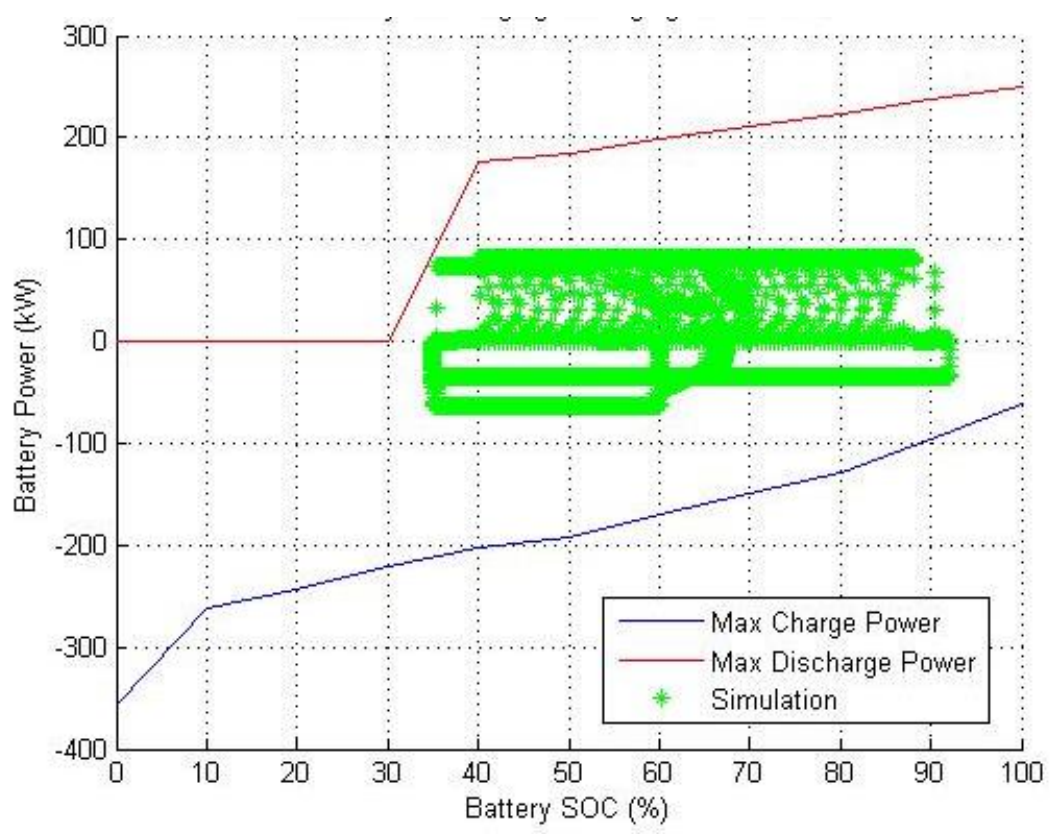

Figure 5.19: ESS activity for parallel HEV model with looking-ahead strategy on down-uphill constant speed driving cycle.

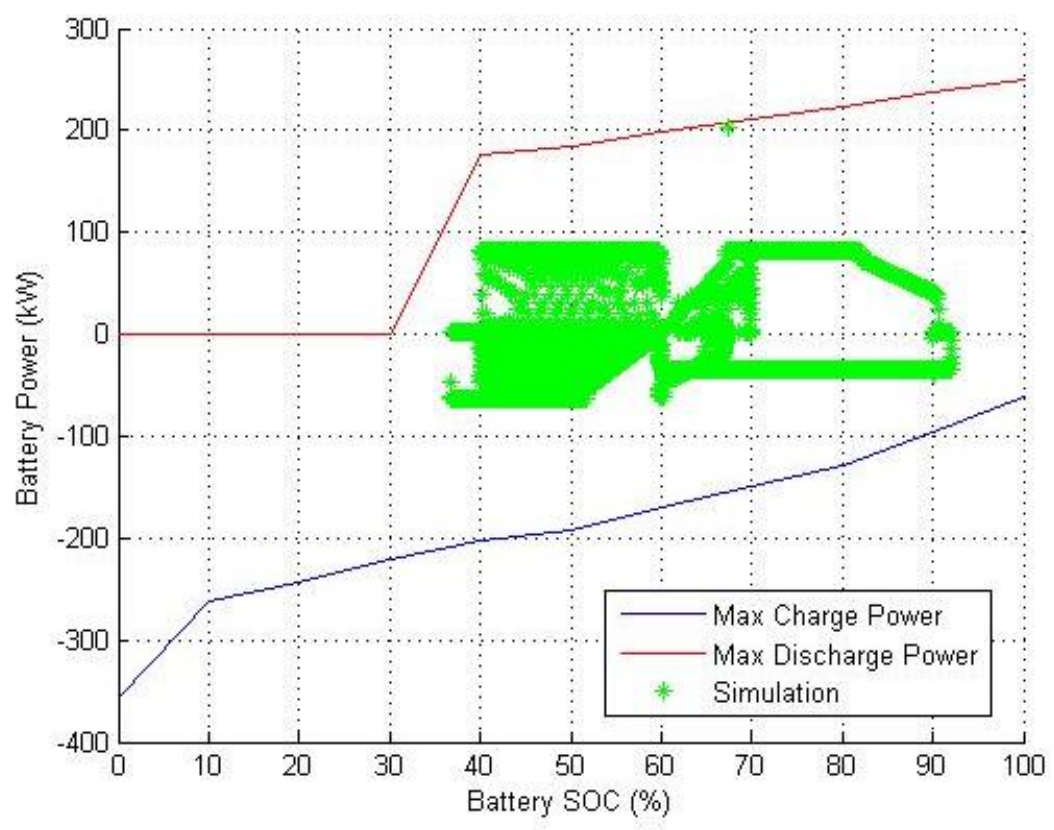

Figure 5.20: ESS activity for parallel HEV model without lookingahead strategy on down-uphill constant speed driving cycle. 


\subsubsection{Real world cycles}

Real world driving cycles have been identified in Chapter 3. These cycles represent the different terrain types (flat, ascending hills and descending hills). Figure (5.21) shows the fuel economy for five different cycles with looking-ahead and without looking-ahead strategy. Figures (5.225.26) show the fuel economy change for different real world cycles and with different scenarios. The suggested scenarios for this study are detailed in Chapter 4. These results were for HEV model with $50 \%$ full load (GVWR $=26,000 \mathrm{~kg}$ ). The results show that the fuel economy for looking-ahead control strategy can be improved up until $3 \%$ in comparison to the same parallel HEV model without looking-ahead strategy. The looking-ahead control strategy benefits on fuel economy improvement were limited on flat terrain type (Clmb2Ind). These results, Figures (5.22 and 5.23), showed also that the precisions of forthcoming traffic information can impact lookingahead response positively. In other words, these results revealed that when the forthcoming traffic information was not precise, then the VPC action regarding the forthcoming power demand might not be prepared properly. This wrong forthcoming power event estimation could either lead to using the engine to charge the ESS with no real need for that or do not guarantee enough capacity in the ESS to capture all free energy. The term Sc stands for scenario, for example, Sc1 means scenario one and so on.

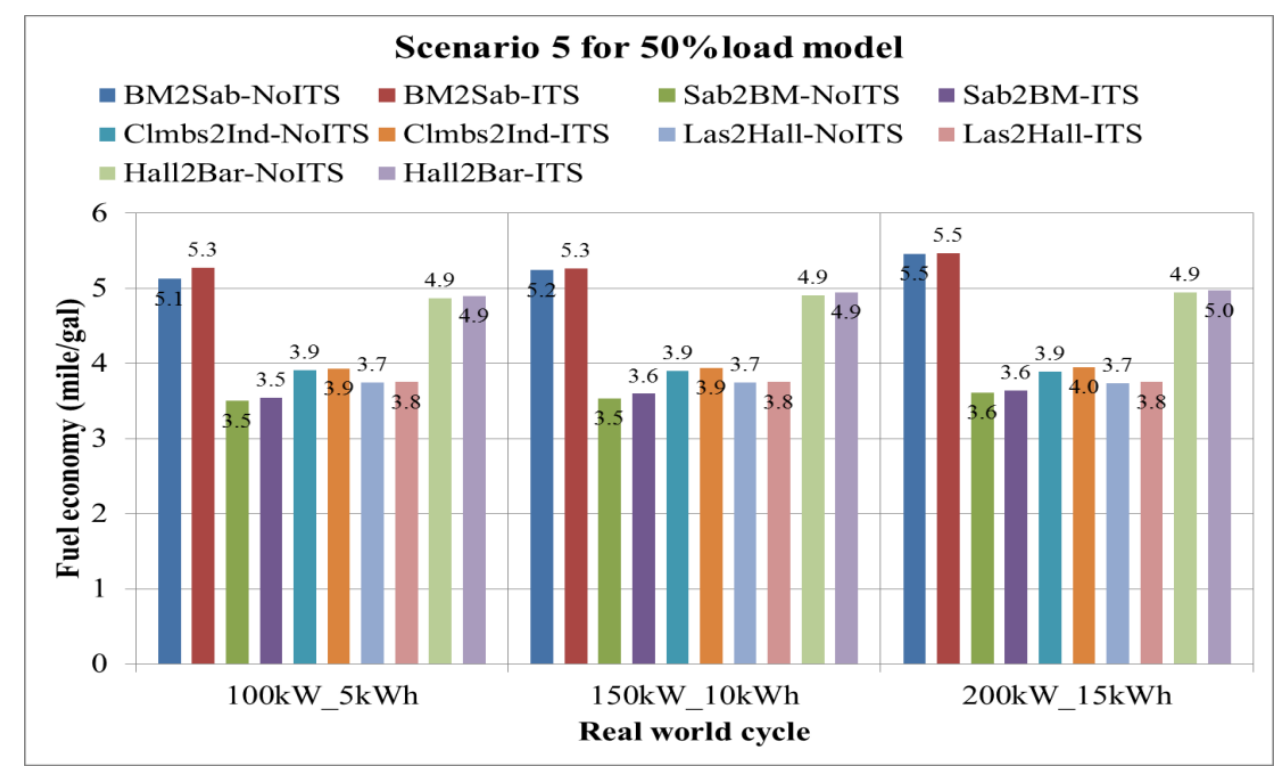

Figure 5.21: Fuel economy for different real world cycles for parallel HEV model (GVWR 26,000 kg) 


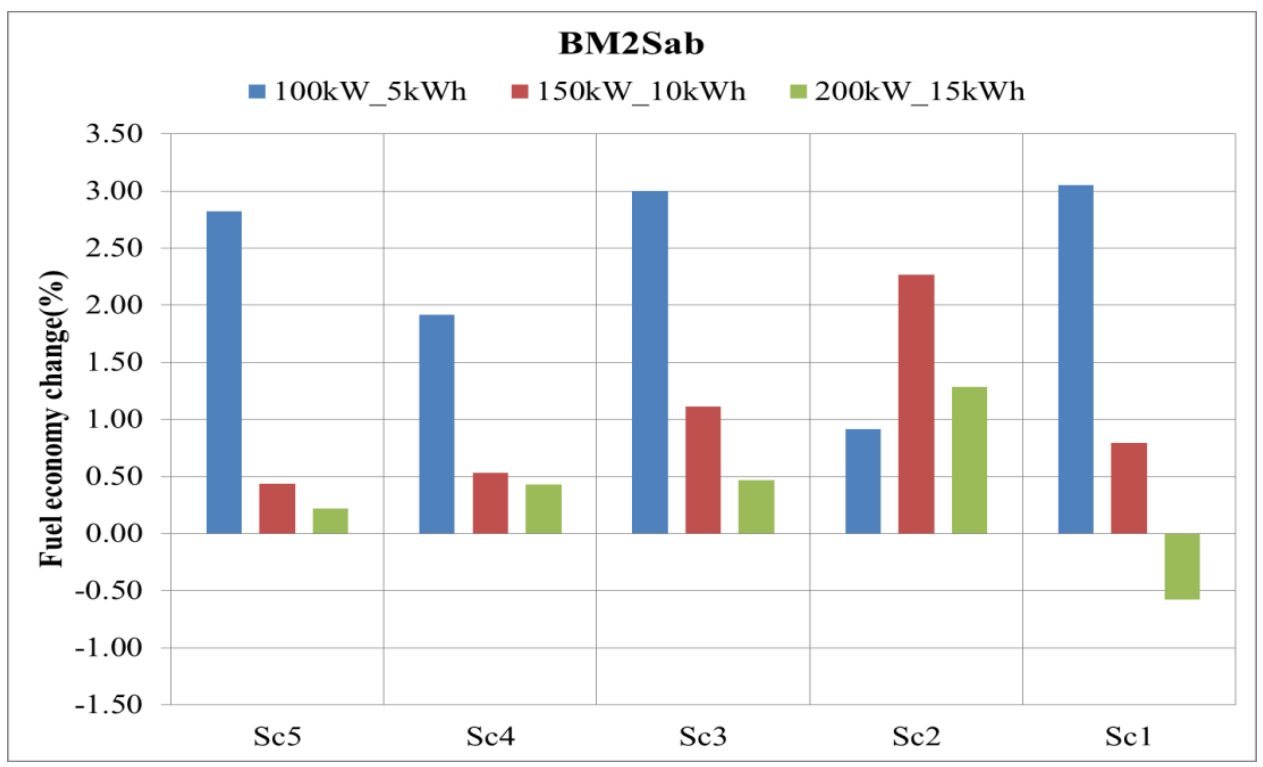

Figure 5.22: Fuel economy change for different scenarios on BM2Sab cycle as a result of the looking-ahead strategy

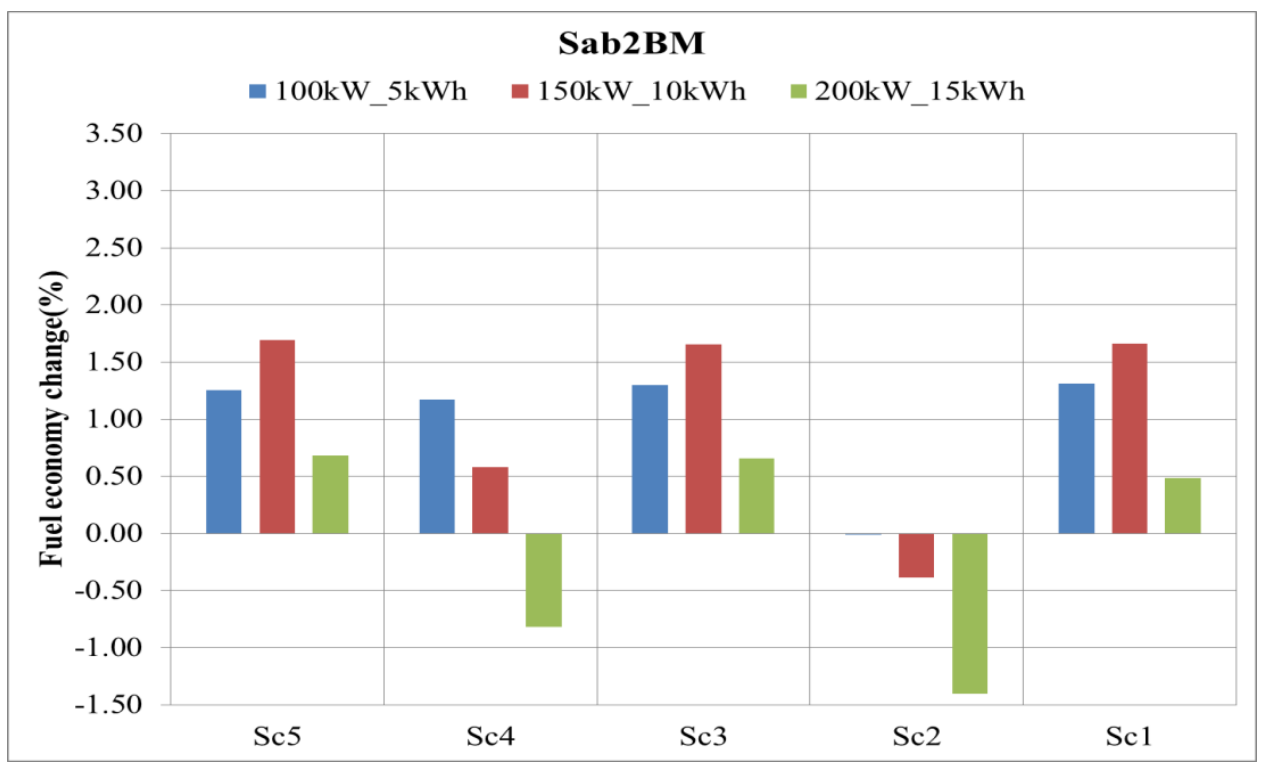

Figure 5.23: Fuel economy change for different scenarios on Sab2BM cycle as a result of the looking-ahead strategy 


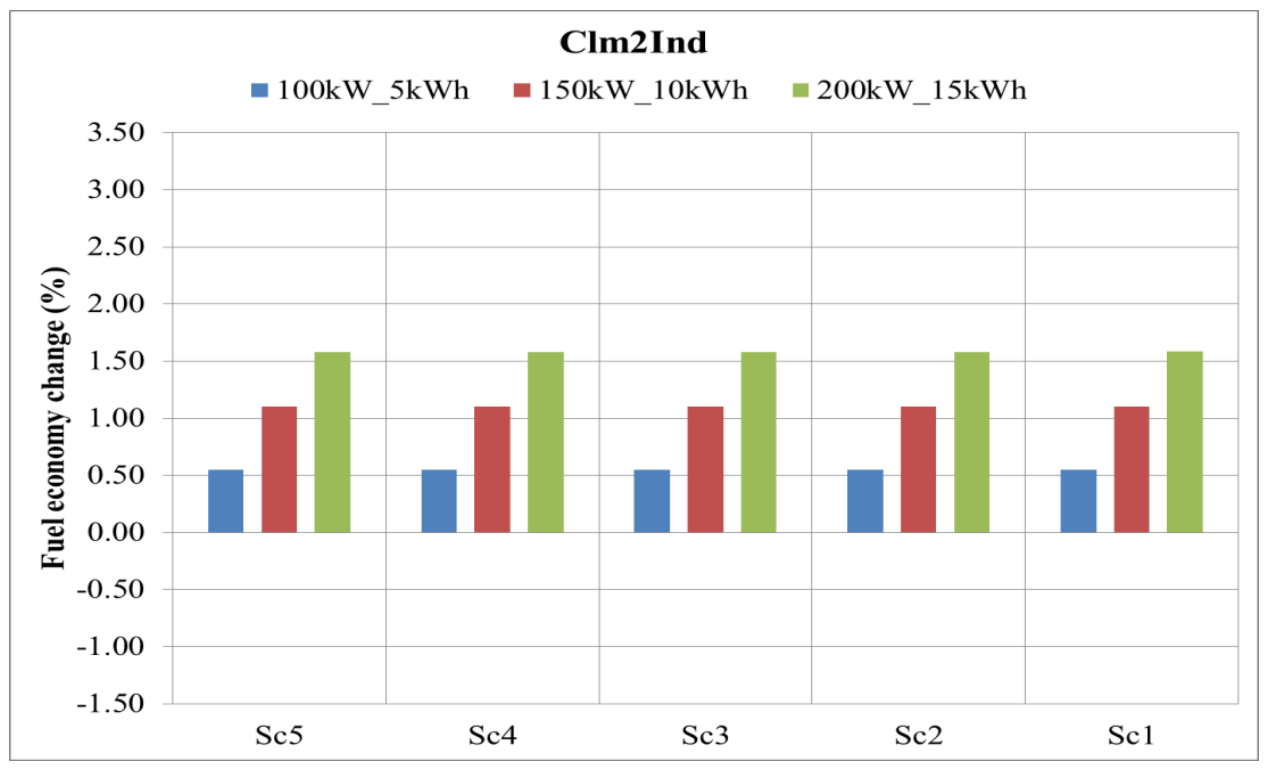

Figure 5.24: Fuel economy change for different scenarios on Clm2Ind cycle as a result of the looking-ahead strategy

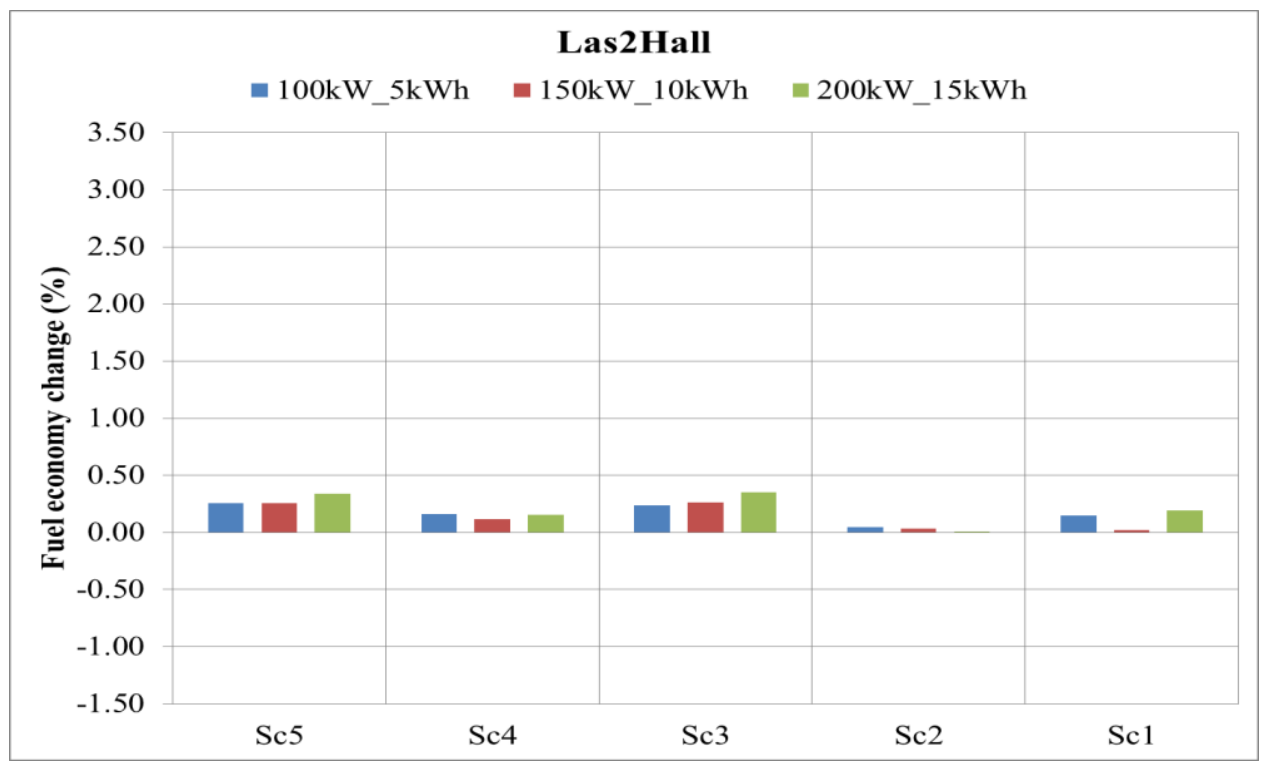

Figure 5.25: Fuel economy change for different scenarios on Las2Hall cycle as a result of the looking-ahead strategy 


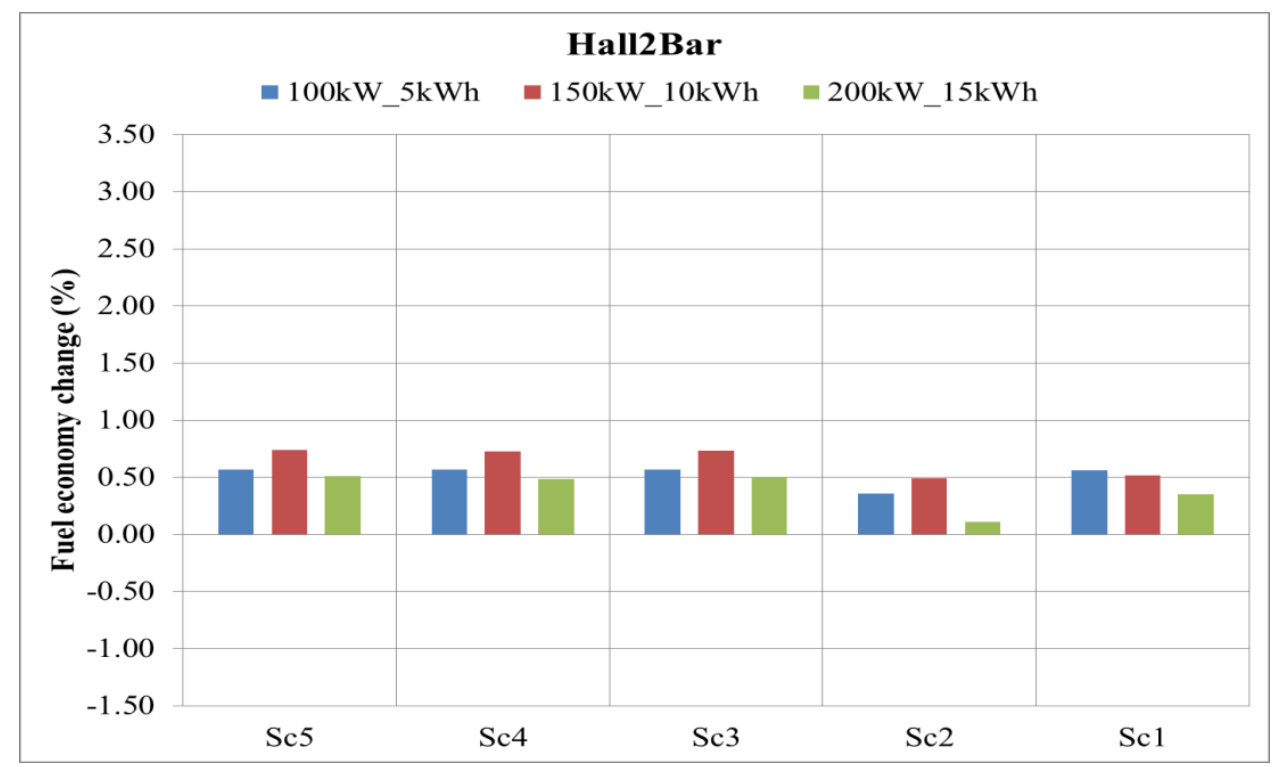

Figure 5.26: Fuel economy change for different scenarios on Hall2Bar cycle as a result of the looking-ahead strategy

Figures (5.27 and 5.28) show the cumulative FC for Sab2BM and BM2Sab real world driving cycles, respectively. These driving cycles represent the different terrain types such as ascending, descending and flat terrains. Moreover, Figures (5.29 and 5.30) show the amount of free energy captured at the battery and captured energy change for looking-ahead strategy in comparison to without looking-ahead control strategy. These results are for parallel HEV model with 100kW and 5kWh electric motor and ESS, respectively, and at 50\% full load model $(26,000 \mathrm{~kg})$. These results revealed that the advanced management of the alternative power source can increase the free energy captured while using the brake system. Moreover, the other important finding of the looking-ahead control strategy is that the improvement of the alternative power source (the electric motor) contribution. 


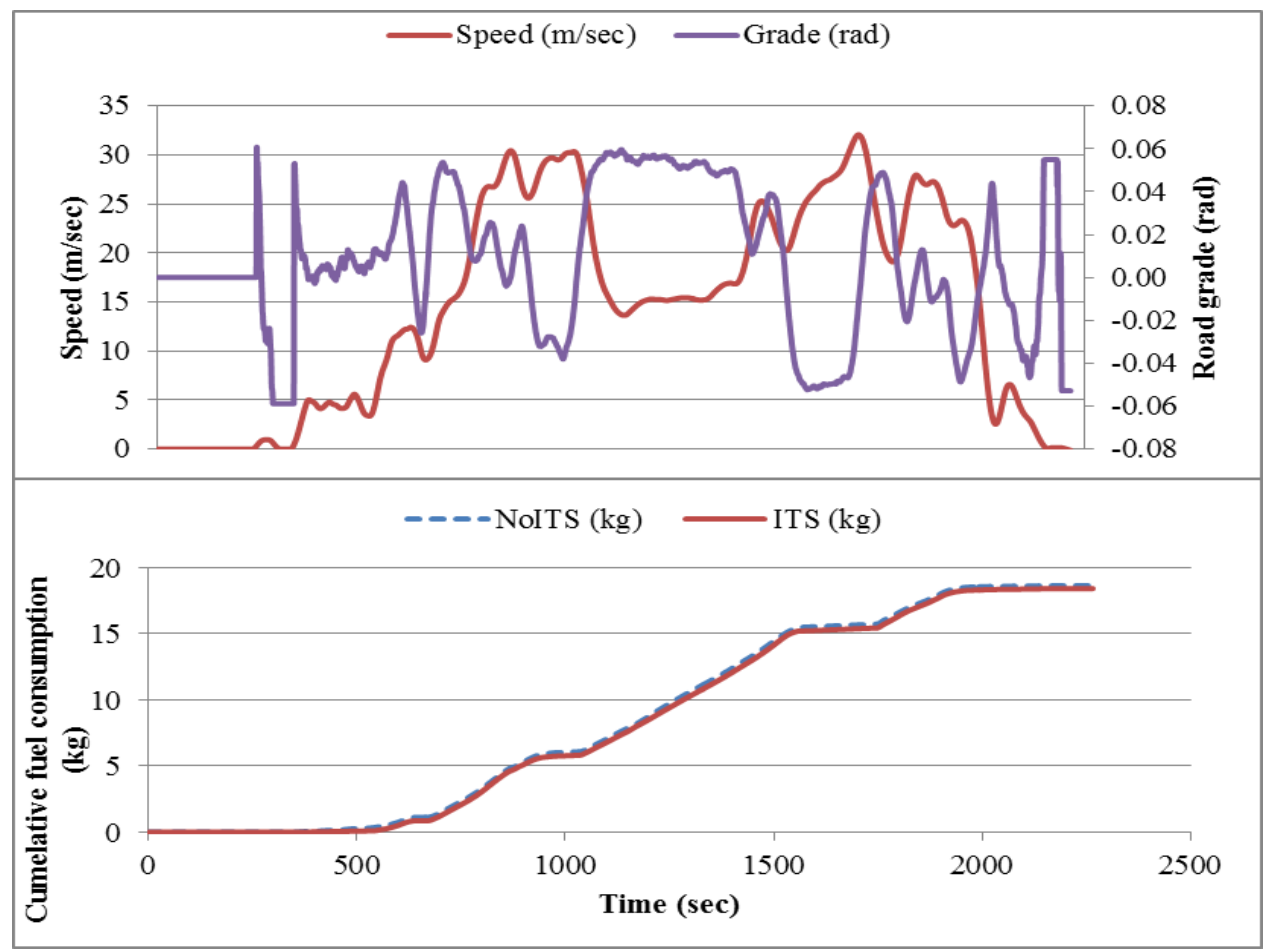

Figure 5.27: Cumulative FC for parallel HEV with and without lookingahead strategy on the real world driving cycle Sab2BM

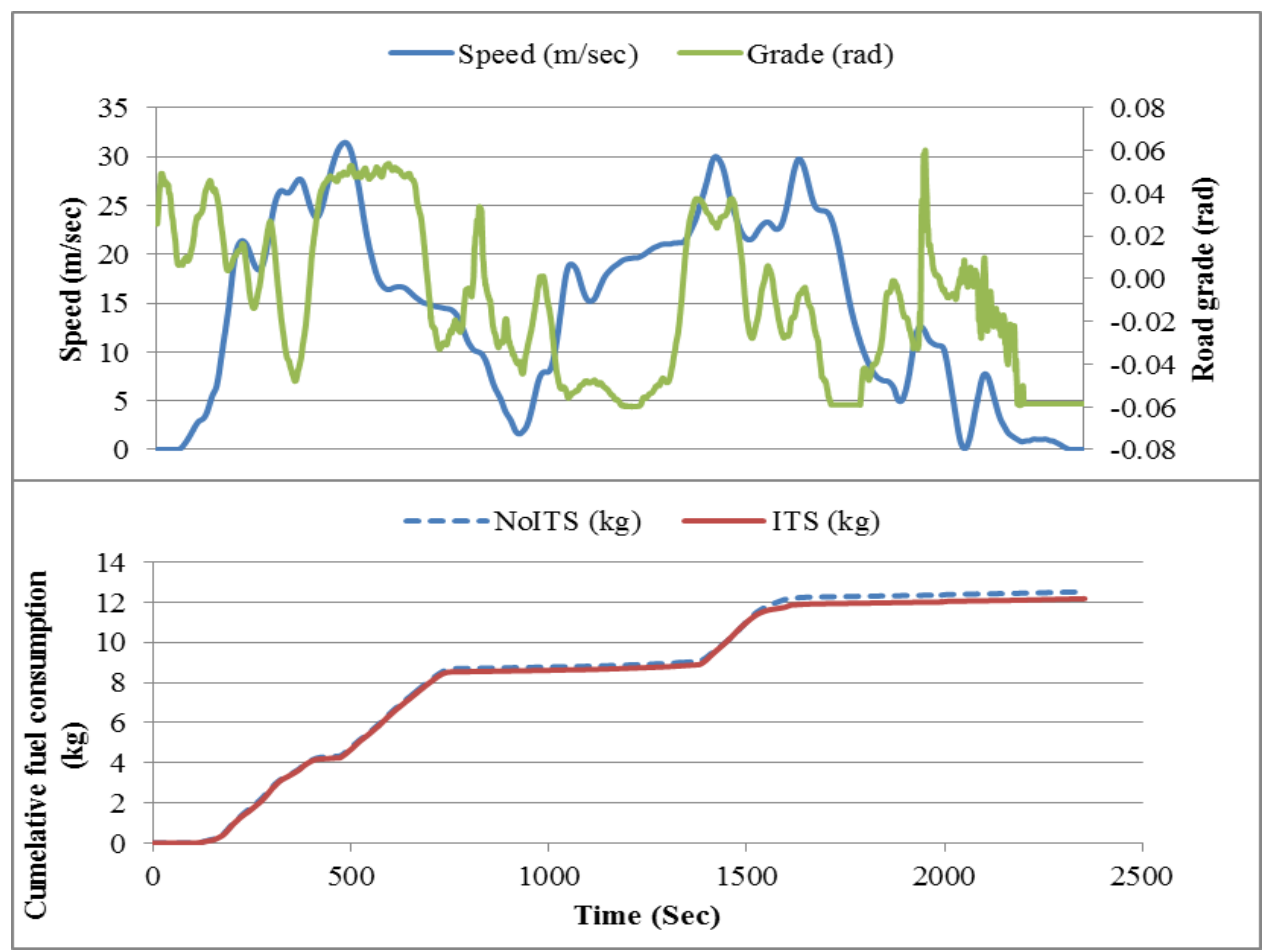

Figure 5.28: Cumulative FC for parallel HEV with and without lookingahead strategy on the real world driving cycle BM2Sab 


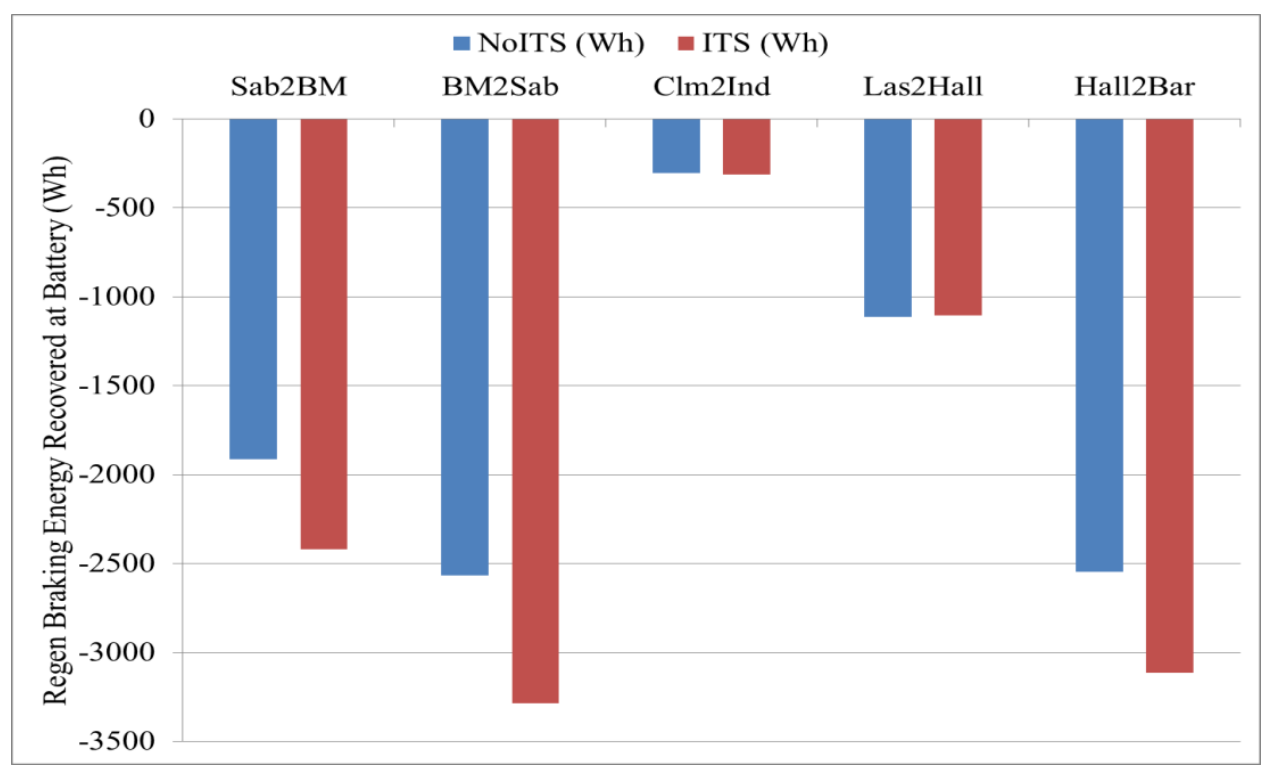

Figure 5.29: The energy recovered at battery using the brake system only on different real world driving cycles

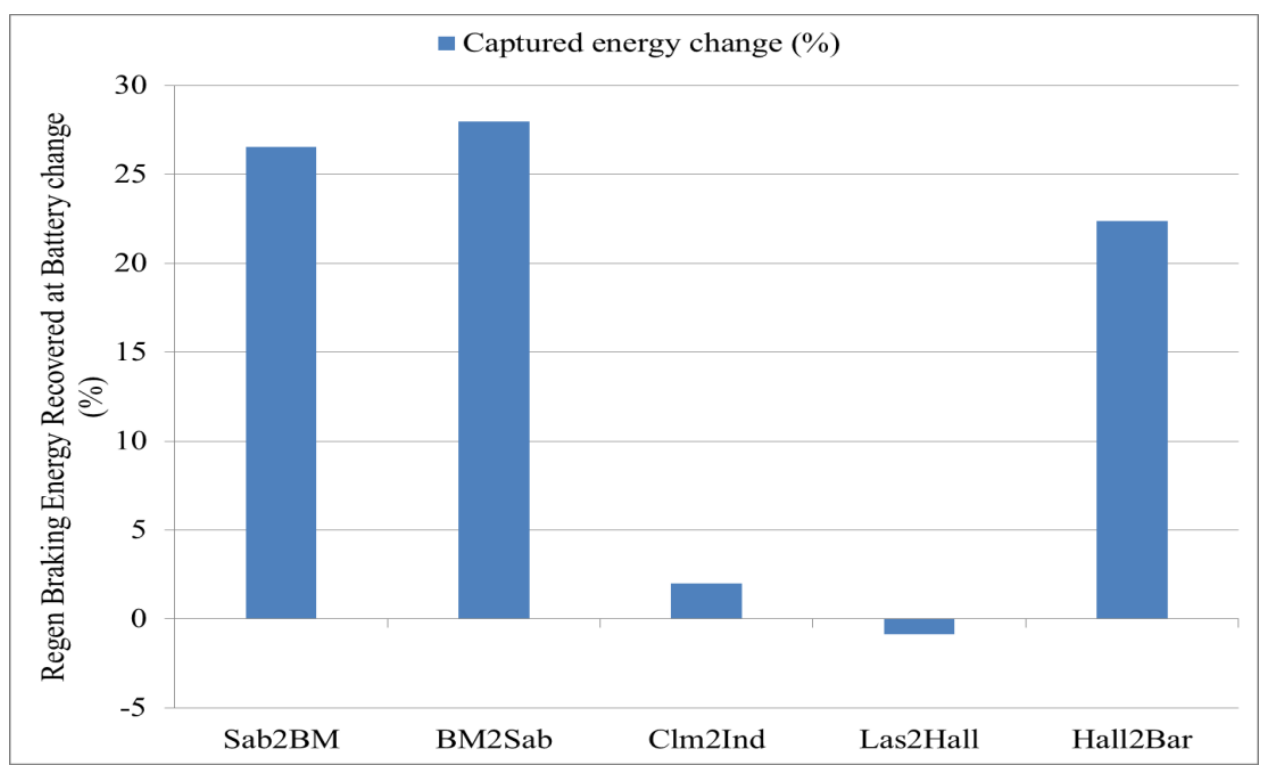

Figure 5.30: Impact of looking-ahead strategy on the amount of free energy recovered at battery for different real world driving cycles 


\subsubsection{Chassis cycles}

The vehicle activities and terrain types may represent the crucial factors affecting improving fuel economy when using a hybrid vehicle instead of a conventional vehicle. Different routes (ascending and descending hills) with constant speed highway cycle and real world driving cycles, including road grade, have been used to investigate the possible benefits of parallel HEV configuration on improving fuel economy for heavy duty vehicle model. In this section, several chassis driving cycles are used. Different scenarios are considered for this investigation. Repeated cruise non-stop driving cycles with different road grades and period of road grade as a sine wave (to mimic hilly and mountain terrain) are used. Repeated HDUDDS driving cycles with different road grades are used here also. Acceleration and deceleration are not detected for these looking-ahead scenarios. Although, for example, in UDDS truck cycle the driver was accelerating during ascending hills, the total power (the engine and the electric motor) was sufficient to track the wheel power demand. A combination of city driving cycles and highway driving cycles (HHDDT65, Transient, UDDS truck, and CSHVR), to mimic the real life truck activities, are used. Figures (5.31 and 5.32) show the fuel economy and fuel economy change for repeated cruise driving cycles with different road grades for parallel HEV (100 kW and $5 \mathrm{kWh}$ for motor and battery) models (GVWR 26,000 kg) with looking-ahead strategy in comparison to parallel HEV without looking-ahead strategy. Figures (5.33-5.36) show the fuel economy and fuel economy change for parallel $\mathrm{HEV}(150 \mathrm{~kW}$ and $10 \mathrm{kWh}$ for motor and battery) models on repeated HDUDDS and combined driving cycles with different road grades and periods of oscillation. Results for repeated HDUDDS and combined driving cycles were for scenario 5, which is looking for practical forthcoming traffic conditions, and 50\% load (GVWR 26,000 kg) vehicle model. The results showed that the highest fuel economy improvement was for the terrain with a maximum road grade of $4 \%$, and the reason for that is because of preparing ESS capacity to capture more energy while using the brake system. However, there are other benefits beside the fuel economy improvement, which is maintaining adequate power during different activities and improve battery pack effectiveness, and these concepts will be discussed later in this chapter. 


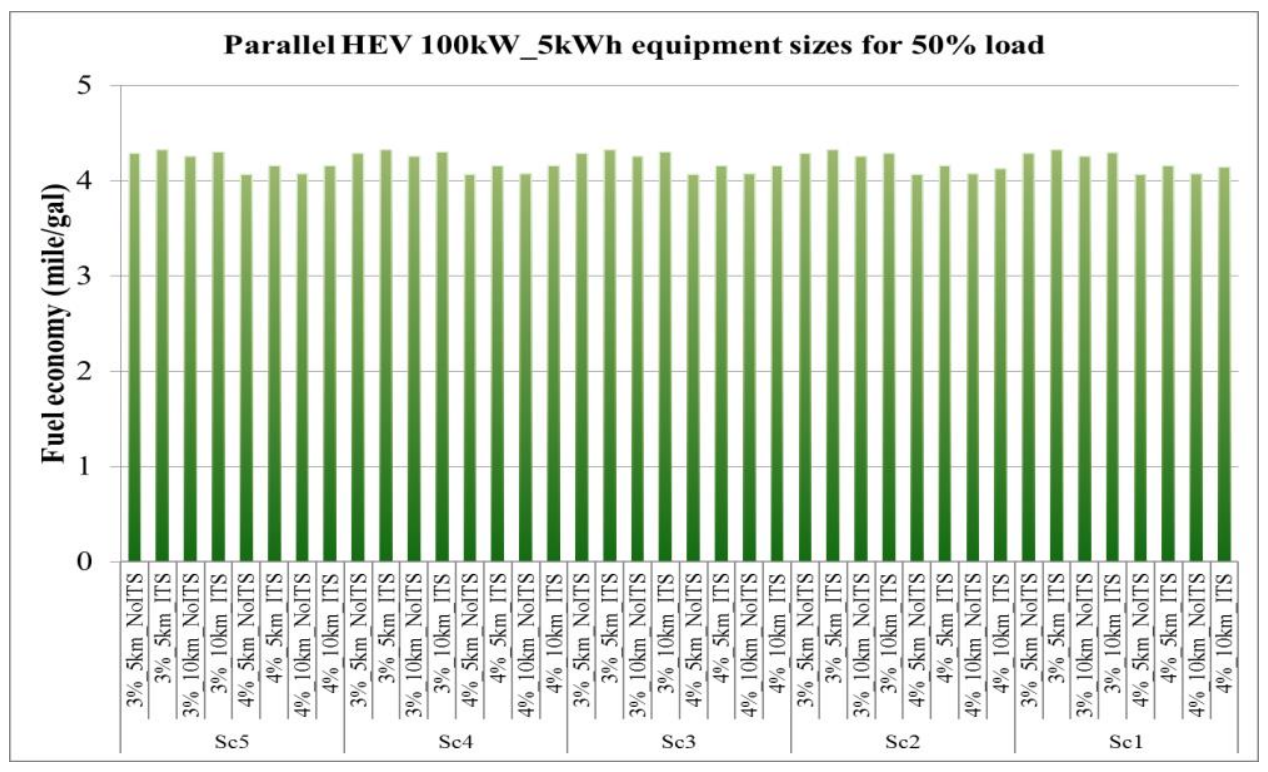

Figure 5.31: Fuel economy for different scenarios on repeated Cruise driving cycle with different road grade and terrain types

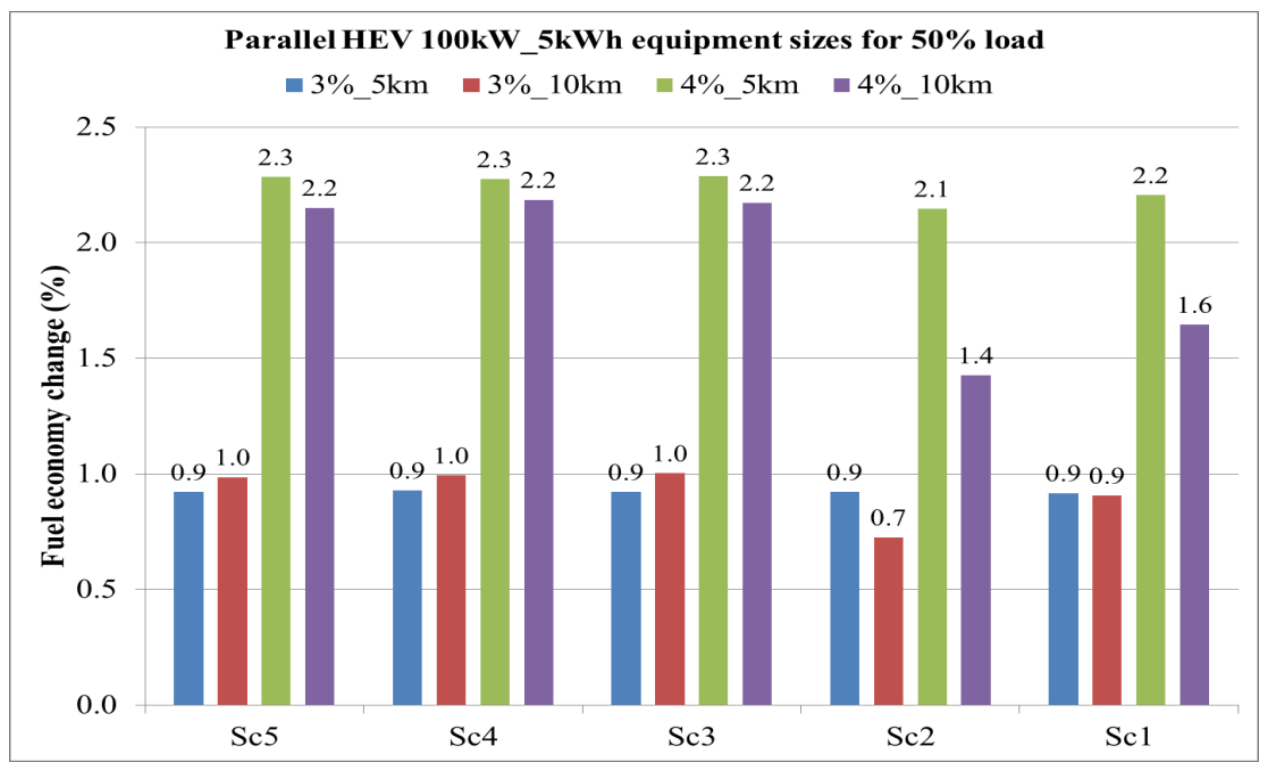

Figure 5.32: Fuel economy change for different scenarios on repeated Cruise driving cycle with different road grade and terrain types 


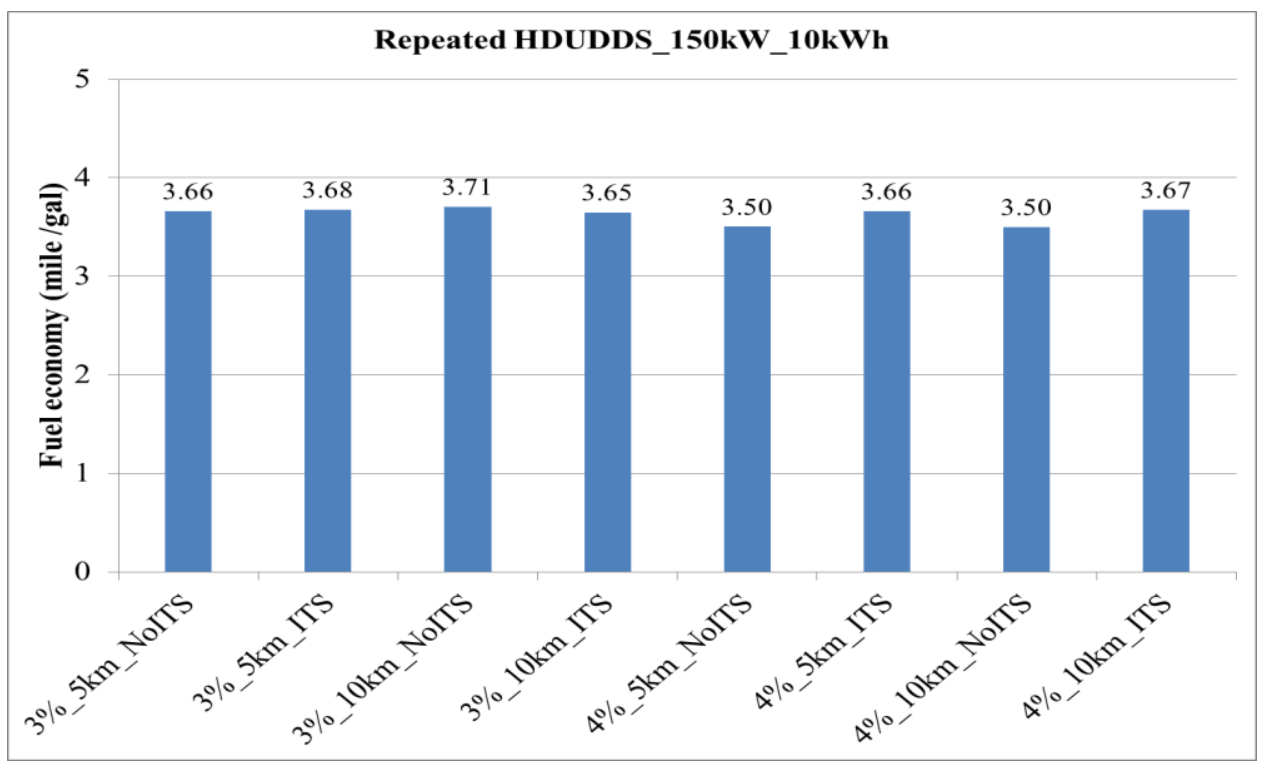

Figure 5.33: Fuel economy for repeated HDUDDS cycle for a parallel HEV (150 kW \& $10 \mathrm{kWh}$ ) model with looking-ahead strategy and without it.

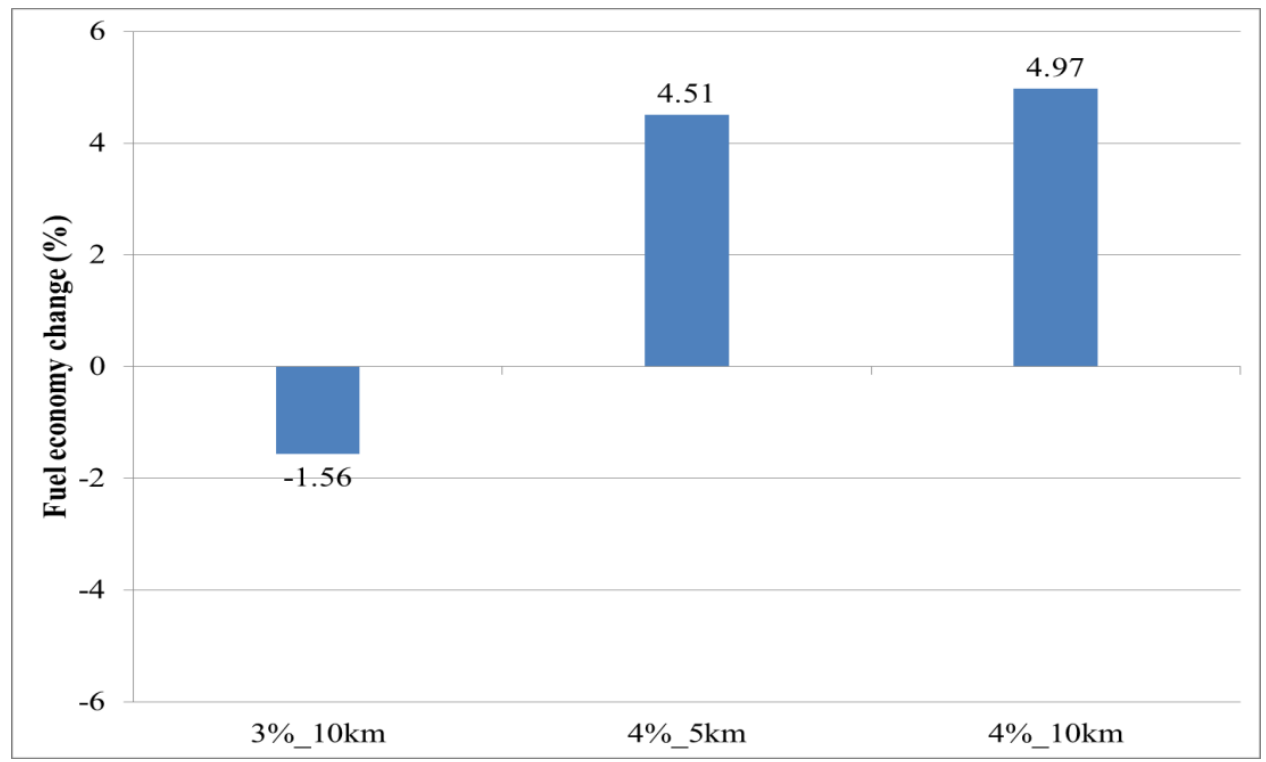

Figure 5.34: Fuel economy change for repeated HDUDDS cycle for a parallel HEV (150 kW \& $10 \mathrm{kWh}$ ) model with looking-ahead strategy and without it. 


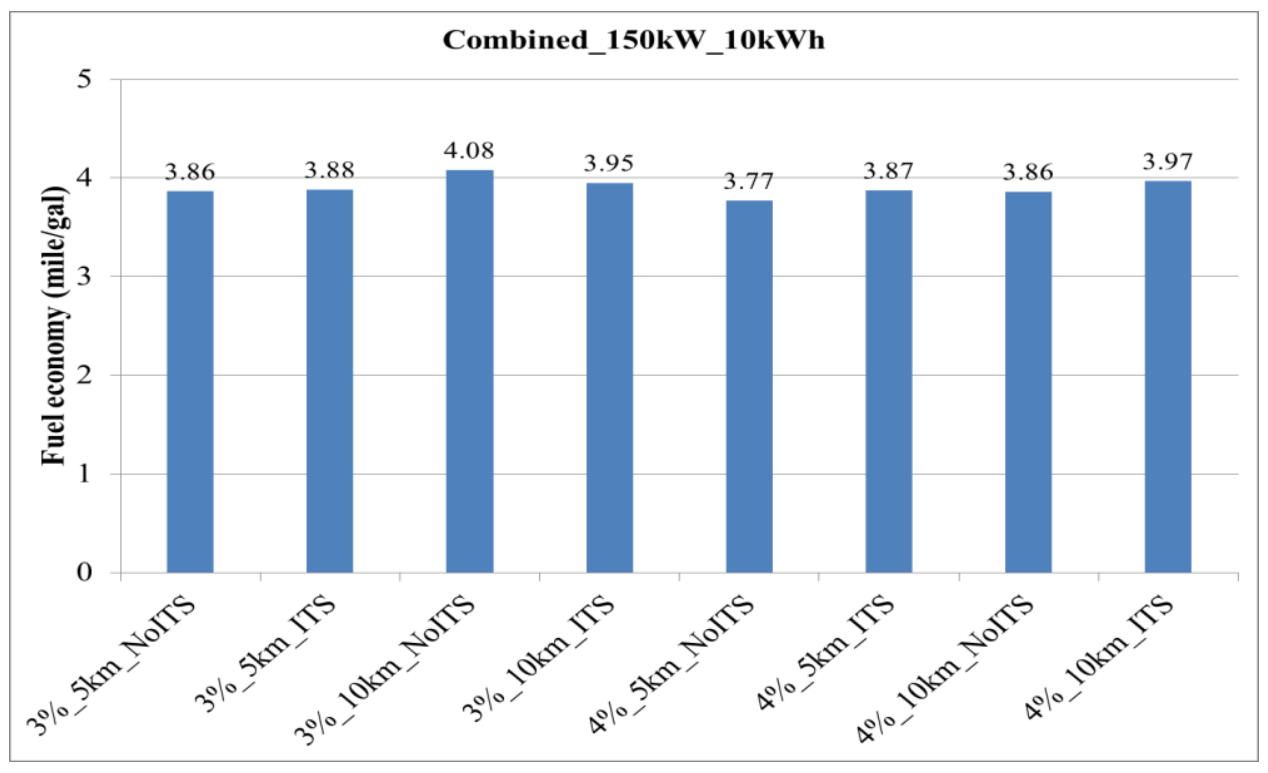

Figure 5.35: Fuel economy for combined driving cycle for a parallel HEV $(150 \mathrm{~kW} \& 10 \mathrm{kWh})$ model with looking-ahead strategy and without it.

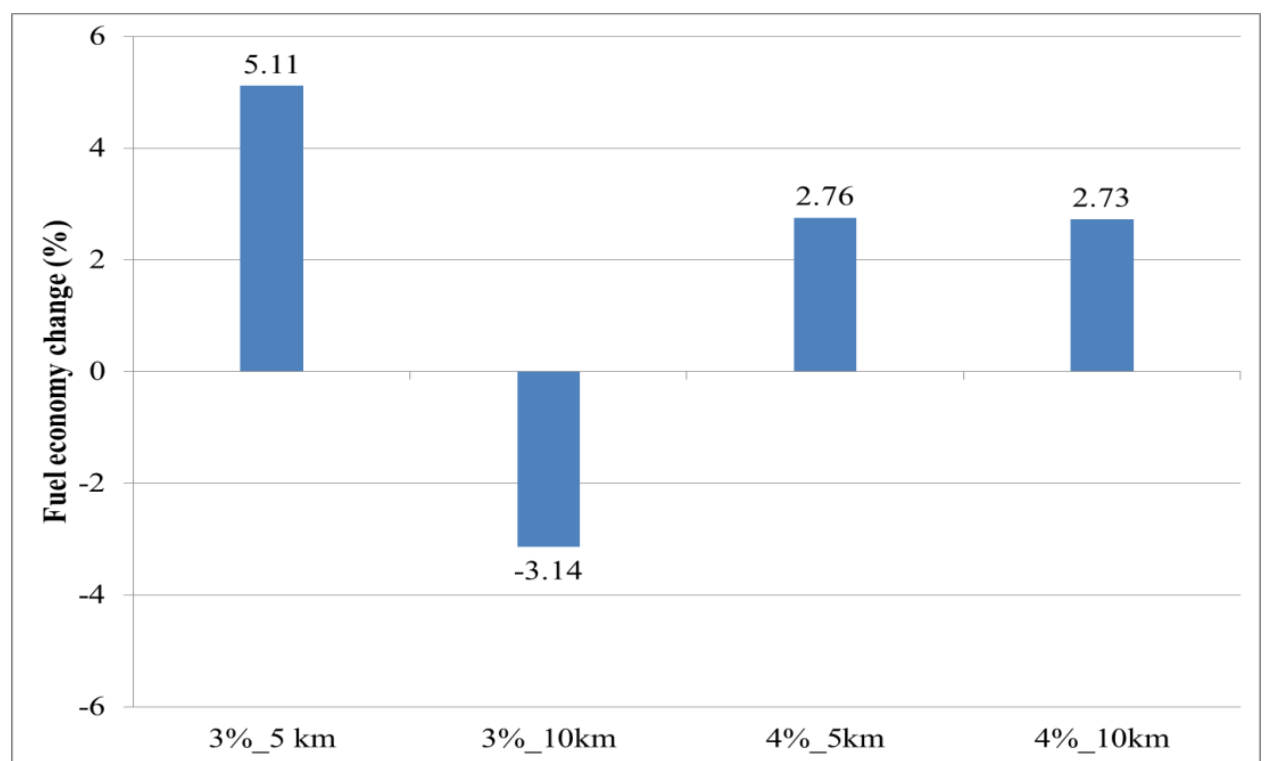

Figure 5.36: Fuel economy change for combined driving cycle for a parallel HEV $(150 \mathrm{~kW} \& 10 \mathrm{kWh})$ model with looking-ahead strategy and without it 


\subsection{The contribution of looking-ahead VPC on energy storage system effectiveness}

Looking-ahead VPC strategy can improve the energy storage effectiveness by guaranteeing as much energy as possible to assist the powertrain system when the vehicle is ascending hills. The ESS effectiveness means here that the capability of the ESS to assist the main power source (the engine) when power demand exceeds the engine peak power or in capturing free energy while using the brake system. On the other hand, looking-ahead VPC strategy can guarantee capacity to capture as much of the expected energy coming from using the brake system as possible. For example, the $5 \mathrm{kWh}$ ESS with looking-ahead strategy can work almost the same as $10 \mathrm{kWh}$ ESS without looking-ahead control strategy as shown in Figure (5.37). For this model with the NiMH (nickel metal hydride) battery type, the safety limits for SOC are limited by $90 \%$ maximum and $30 \%$ minimum [72]. Therefore, the actual energy capacity available of the battery is about $60 \%$ of the total battery size. Figure (5.37) shows the capacity available for charging or discharging with both (with looking-ahead and without looking-ahead) control strategies. For the proposed strategy, the VPC will discharge the battery, based on the total upcoming free energy, down until $35 \%$ if there is expected energy capturing. Moreover, the VPC will charge the battery, based on the total upcoming extra energy (exceeding engine power) needed, up until $85 \%$ if there is expected extra power demand due to ascending a hill.

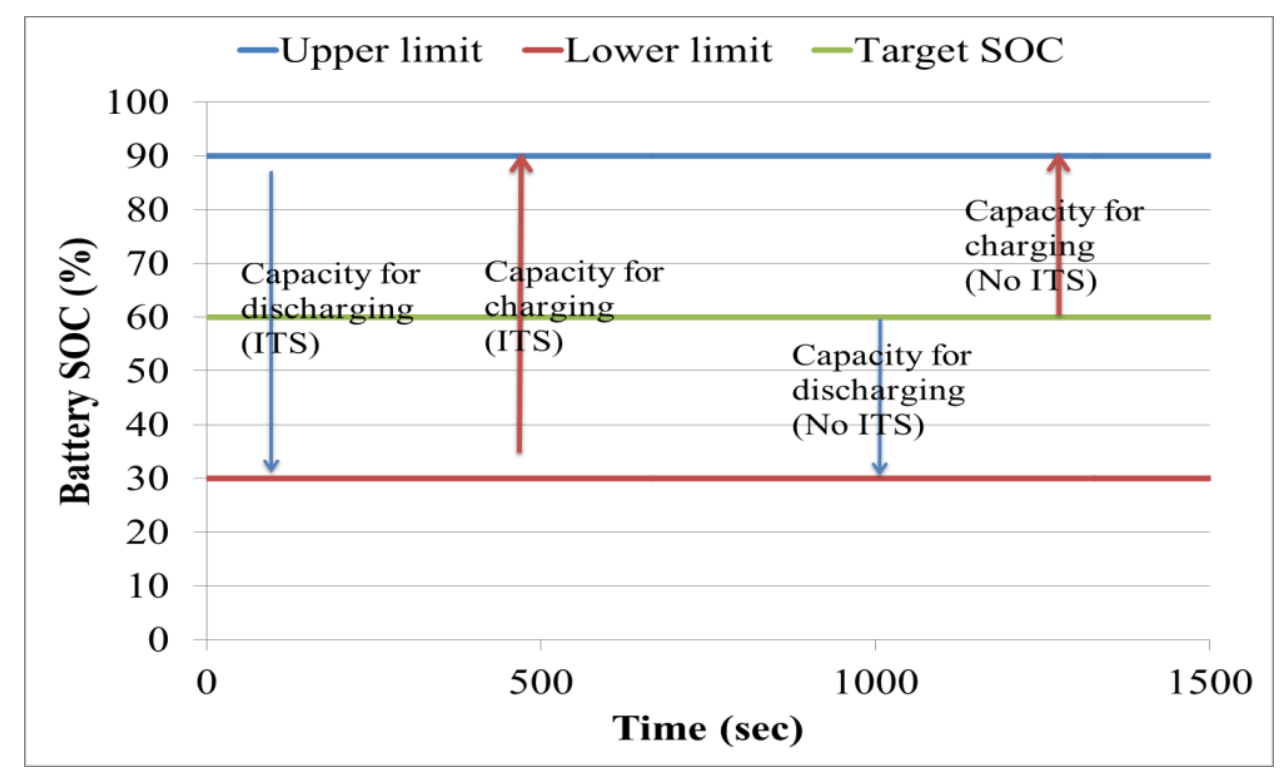

Figure 5.37: The battery capacity for charging and discharging with and without looking-ahead strategy. 
Since the battery system represents the alternative power source for a hybrid electric vehicle, any increase in effective capacity of it can contribute the HEV effectiveness, except that it can add mass to the vehicle. For the conventional parallel hybrid electric vehicle the default battery SOC represents $50 \%$ of actual battery capacity. VPC for a HEV is sustaining battery SOC at the middle. On the other hand, in looking-ahead VPC strategy the battery SOC will be adopted based on the power demand. The major objectives of this strategy are to guarantee as much as possible capacity for charging and discharging the battery system and to avoid using the engine to charge the battery, unless there was a need for that to avoid charging efficiencies losses. To show the obvious impact of control strategy on the battery effectiveness, the model was tested on long, to use all the ESS capacity, ascending or descending hills. Figures (5.38-5.40) show the battery SOC variation for pre transmission parallel HEV with $100 \mathrm{~kW}$ and $5 \mathrm{kWh}$ equipment sizes for the electric motor and the battery respectively at 50\% full load and for scenario 5, which is looking for typical forthcoming traffic conditions, on different terrain types with constant speed cycle (about $60 \mathrm{mph}$ ). In Figure (5.39), at peak battery SOC there was a saw tooth pattern and it due to VPC response. In other words, the VPC stops charging the battery when battery SOC reaches the upper safety limit, and then the battery energy depletes due to electric accessories load (3 kW for this model); so the VPC allows charging it using free energy and so on.

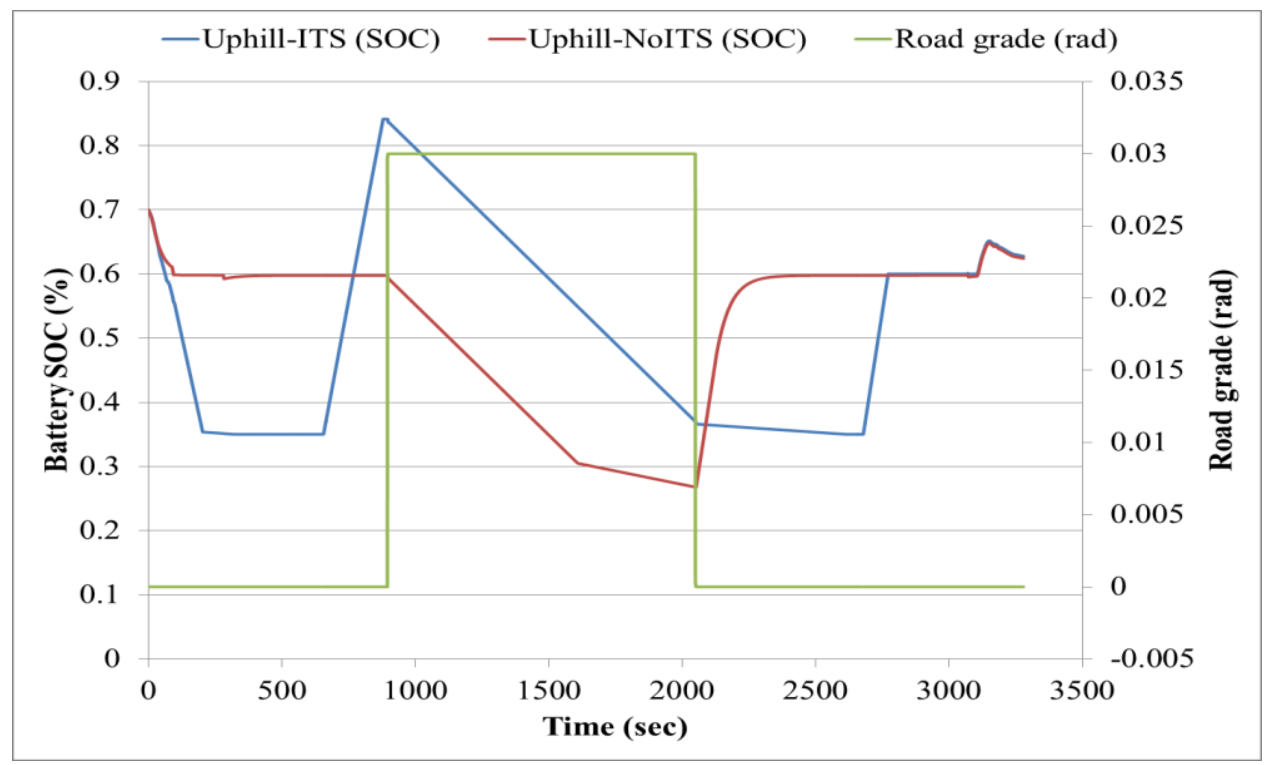

Figure 5.38: Energy storage system effectiveness on ascending hillhighway driving cycle with ITS and No ITS strategy 


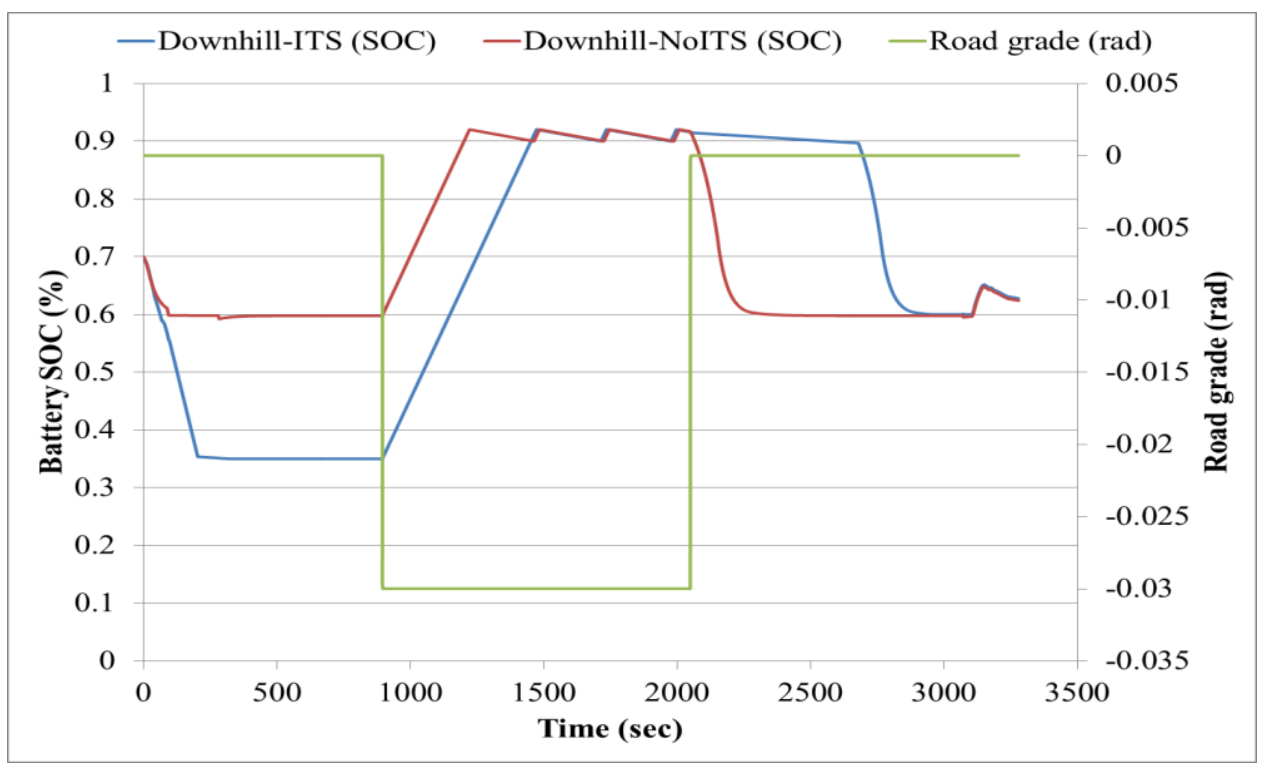

Figure 5.39: Energy storage system effectiveness on descending hillhighway driving cycle with ITS and No ITS strategy

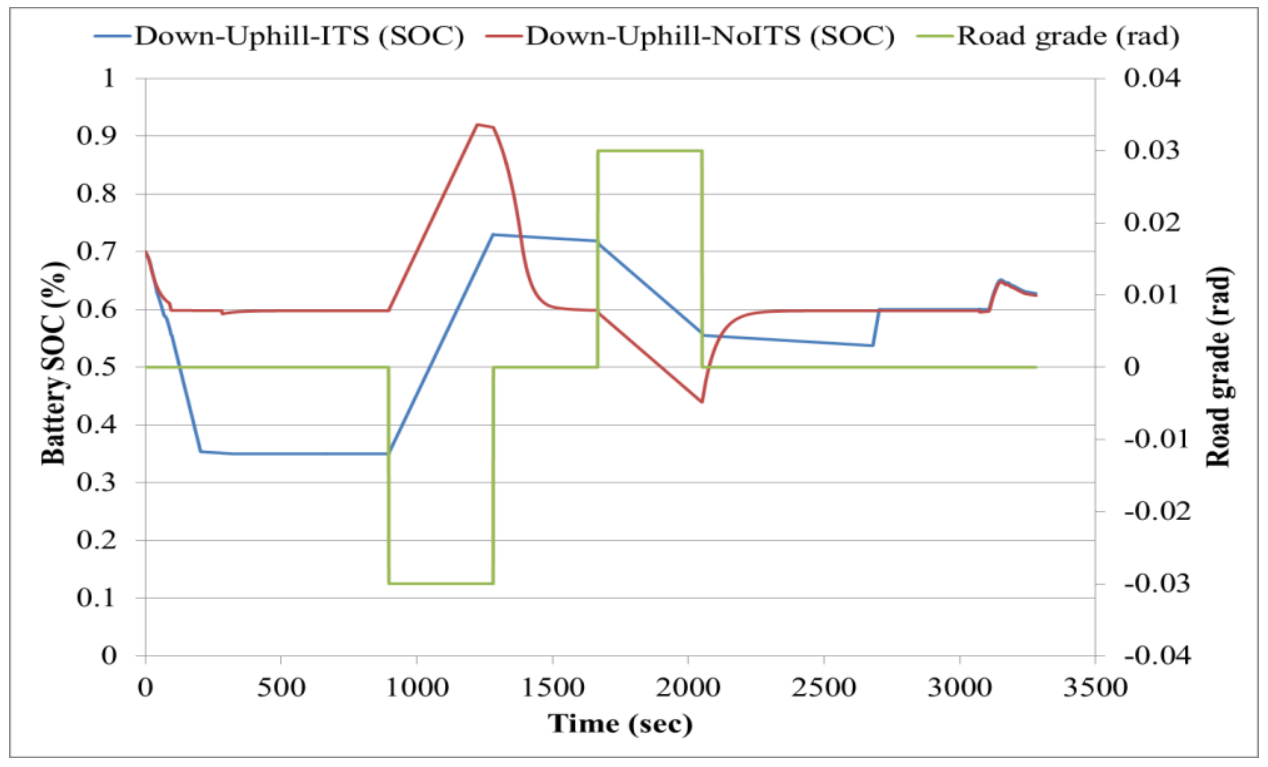

Figure 5.40: Energy storage system effectiveness on descending-ascending hill-highway driving cycle with ITS and No ITS strategy 


\subsection{The ability of suggested control strategy to improve maintaining adequate power during driving}

For a conventional powertrain, if the vehicle power demand surpasses the peak engine power, the VPC will compromise the vehicle speed to make the power demand match the peak engine power available. However, in the hybrid electric system there is a secondary power source which is the electric motor, and the electric motor may improve total power sources. The amount of assistance the hybridization system may be able to offer to the powertrain depends on the equipment (i.e. electric motor and battery) sizes. Since the default strategy for the VPC is to maintain the battery SOC at $60 \%$, the chance for the hybridization system to assist the engine may be (on a long ascending hill) limited. However, the looking-ahead control strategy can override this problem by preparing the assistance power ahead. The preparing can be by charging the battery (using the engine) with approximately the same as expected extra energy needed. The limitation to prepare the system completely for the forthcoming power demand is the upper limit of battery SOC. Moreover, the other limitations for looking-ahead strategy benefits might be the unexpected driver behavior (such as driving on different route) or wrong calculation of power demand based on wrong road grade estimation. This improvement in the vehicle's total power will be at the expense of fuel economy and that is due to charging and discharging in efficiencies. To investigate the impact of this strategy obviously on sustaining power, the ascending and descending hill-constant speed cycles represents the best example to show. Figures (5.41-5.44) show the impact of the looking-ahead control strategy for conventional vehicle in comparison to hybrid electric vehicle with and without looking-ahead control strategy. These results are for $100 \%$ full load model $(\mathrm{GVWR}=36,000 \mathrm{~kg})$ with equipment sizes of $150 \mathrm{~kW}$ and $10 \mathrm{kWh}$ for the electric motor-generator and battery pack respectively. The preliminary results for this strategy revealed that the looking-ahead can improve HEV in comparison to HEV without looking-ahead strategy. For example, in the Figure (5.42) the engine power demand show different responses at about 2100 seconds of the trip time because of the looking-ahead strategy. The parallel HEV model without ITS worked immediately on charging the ESS and maintained the SOC at the middle, but the parallel HEV with ITS didn't request immediate charging because there was no need for the forthcoming events. 


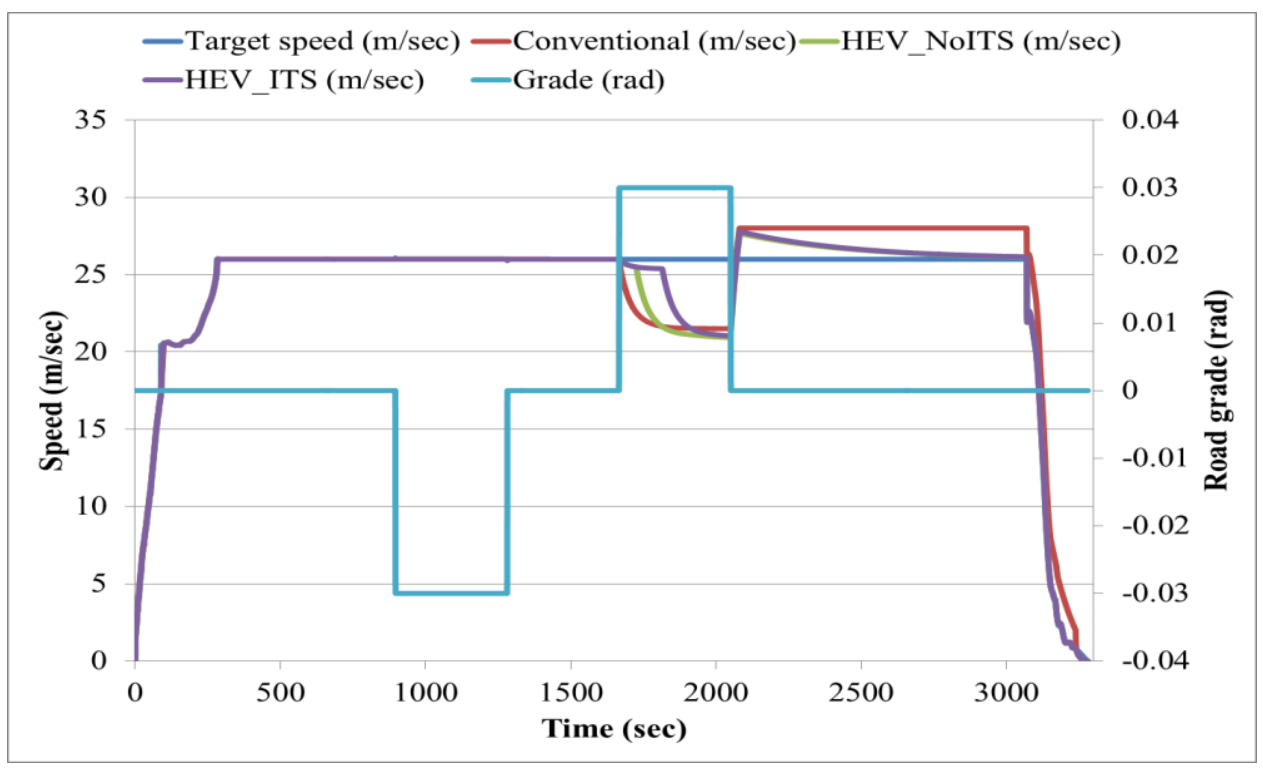

Figure 5.41: Vehicle speed for different models of $100 \%$ full load truck on down-uphill cycle

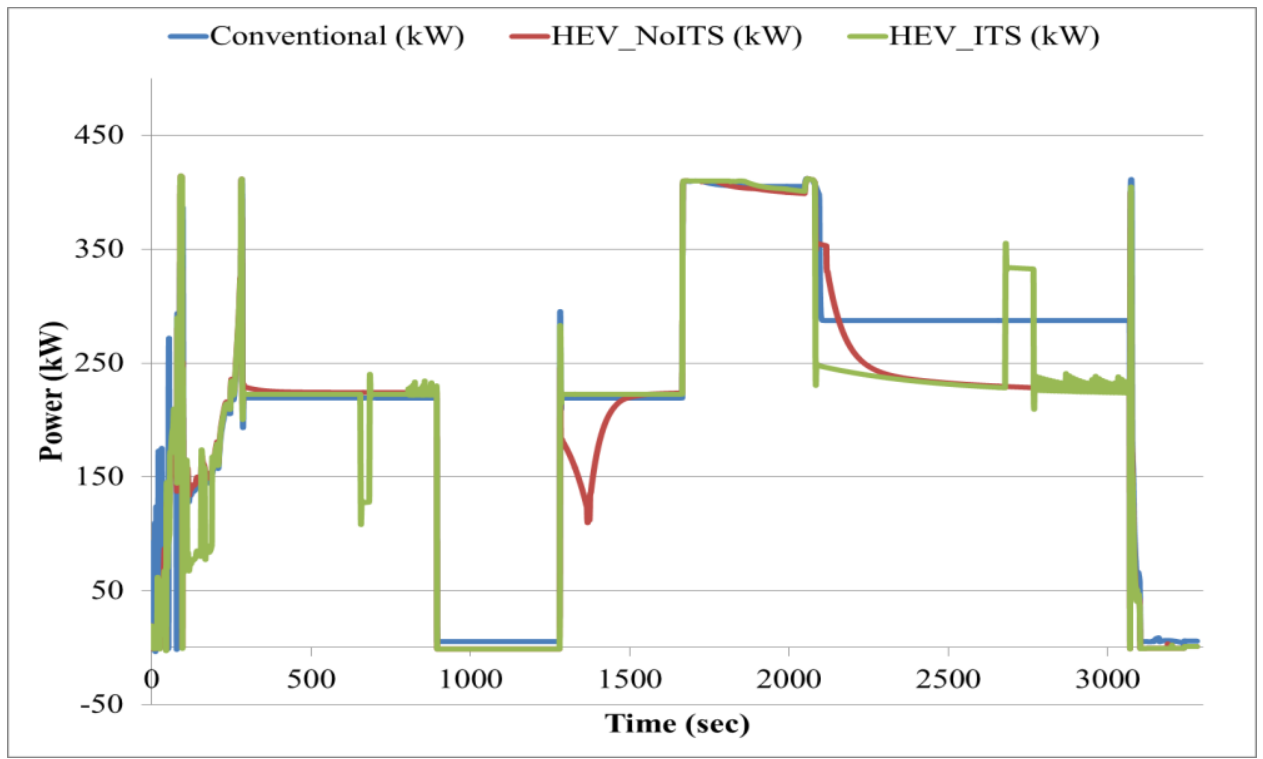

Figure 5.42: Engine power for the different vehicle models with $100 \%$ full load on down-uphill cycle 


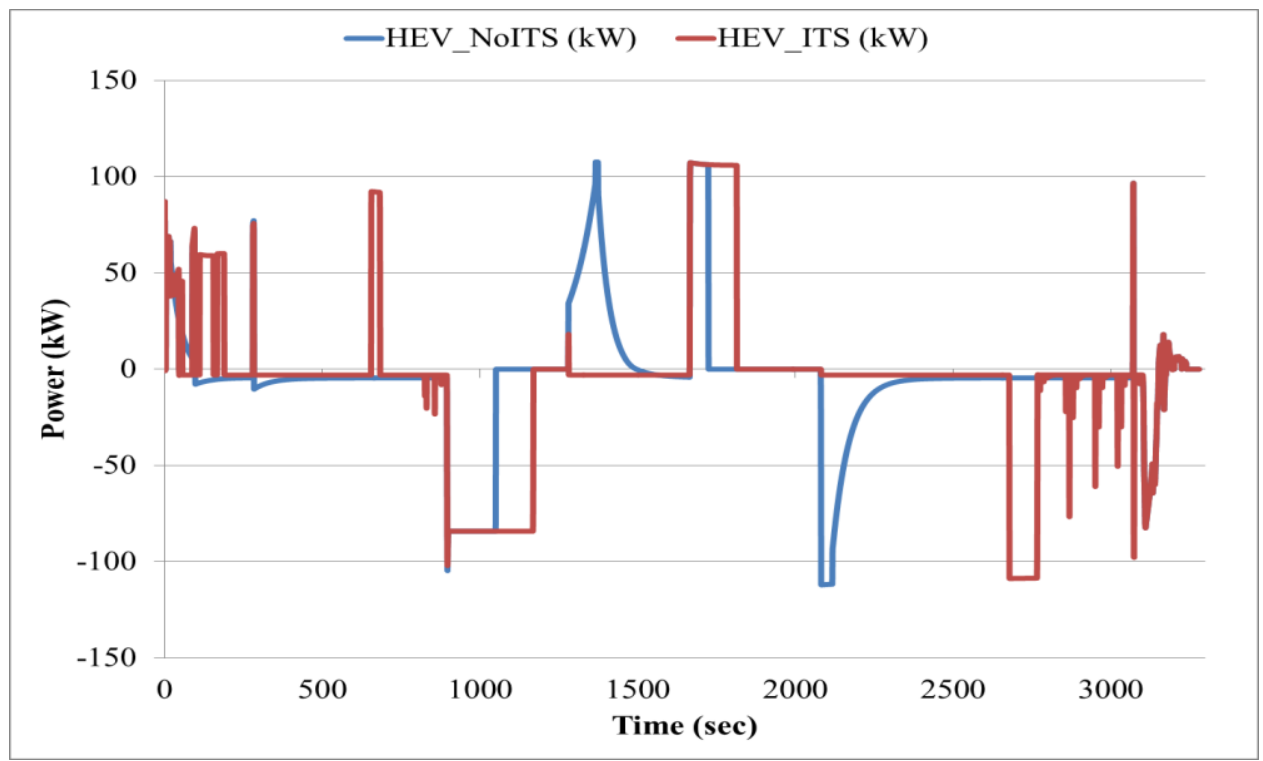

Figure 5.44: Electric motor power for HEV model with and without looking-ahead strategy with $100 \%$ full load on down-uphill cycle

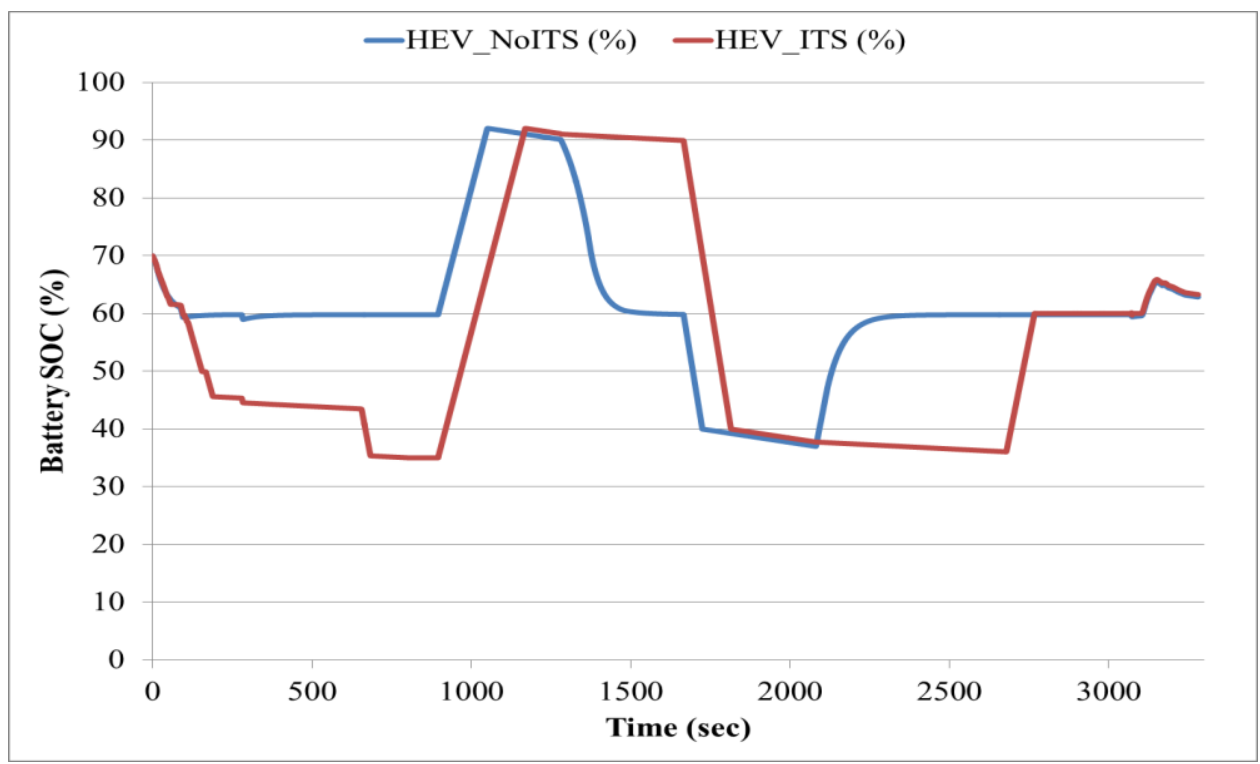

Figure 5.43: Battery SOC for HEV model with and without looking-ahead strategy with $100 \%$ full load on down-uphill cycle 


\subsection{Looking-ahead strategy impact on maintaining adequate power for different real world driving cycles.}

Maintaining adequate power can be investigated by comparing the vehicle's model speed to the target vehicle speed on different driving cycles. Different real world cycles, including road grade data, were used in this investigation, and these driving cycles represent ascending hills, descending hills, and flat terrain highway cycles. For real world driving cycles, will see different terrain types. For example, hilly terrain and mountain terrain with small (i.e. $-2 \%$ to $+2 \%$ for heavy duty vehicles) and steep (less than $-5 \%$ or more than $5 \%$ ) road grade can be noticed in the different real world cycles.

Figures (5.45-5.49) show the vehicle speed for different real world driving cycles. The vehicle model is $100 \%$ full load $(36,000 \mathrm{~kg})$, and the hybridization equipment sizes are $150 \mathrm{~kW}$ and 10 $\mathrm{kWh}$ for the electric motor-generator and battery respectively. These results show that the powertrain's power (engine and hybridization equipment) was sufficient to maintain vehicle speed even without looking-ahead control strategy. However, it may be noticed that a significant impact of looking-ahead control strategy is the opportunity to downsize the engine or equipment sizes (next section). The HEV with both control strategies was able to maintain vehicle speed (sufficient power). However, the conventional vehicle failed to maintain vehicle speed (sufficient power) at ascending hills terrain.

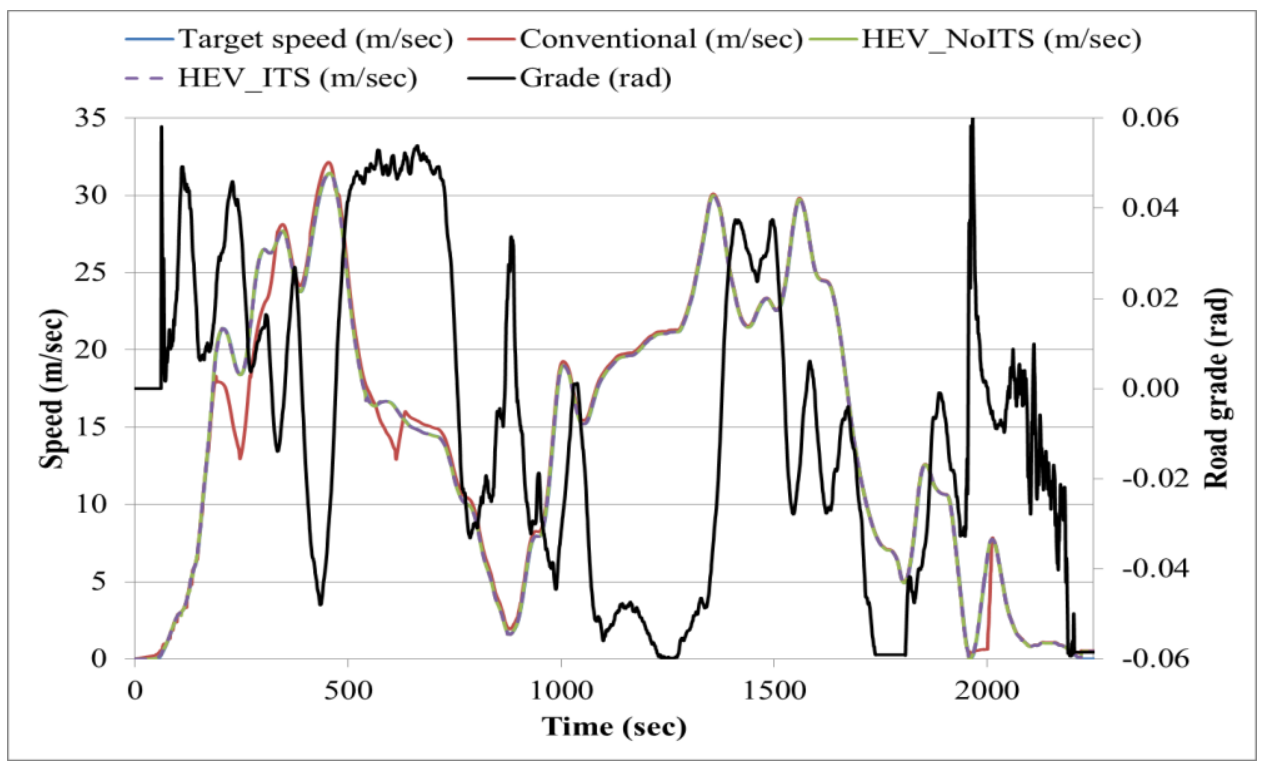

Figure 5.45: Impact of looking-ahead control strategy on maintaining vehicle speed on BM2Sab cycle 


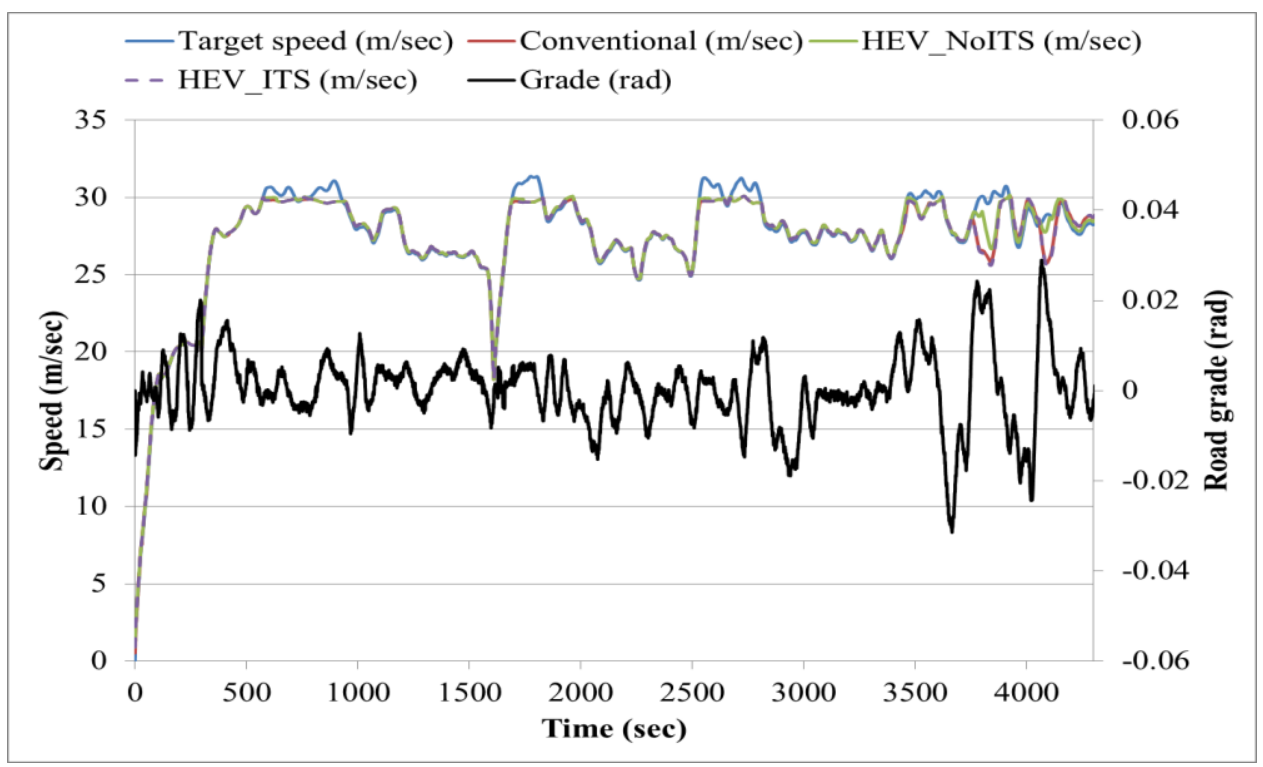

Figure 5.46: Impact of looking-ahead control strategy on maintaining vehicle speed (GVWR 36,000 kg) on Clm2Ind cycle

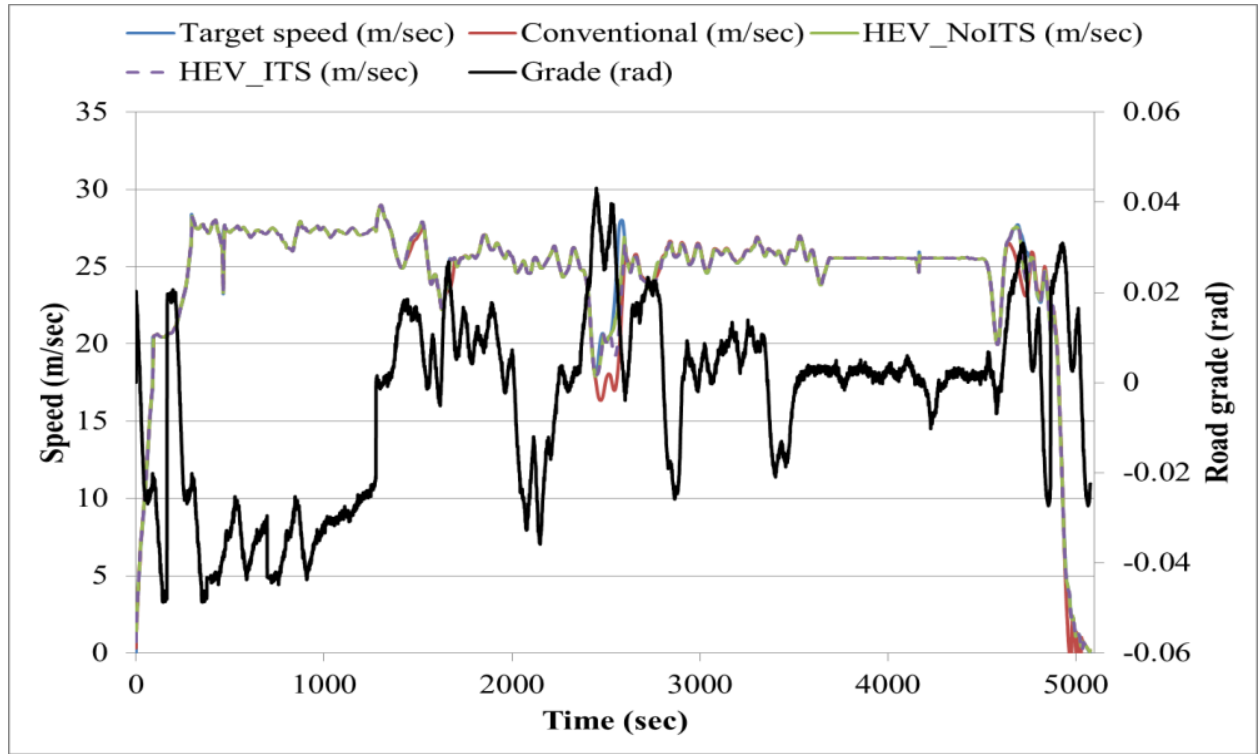

Figure 5.47: Impact of looking-ahead control strategy on maintaining vehicle speed (GVWR 36,000 kg) on Hall2Bar cycle 


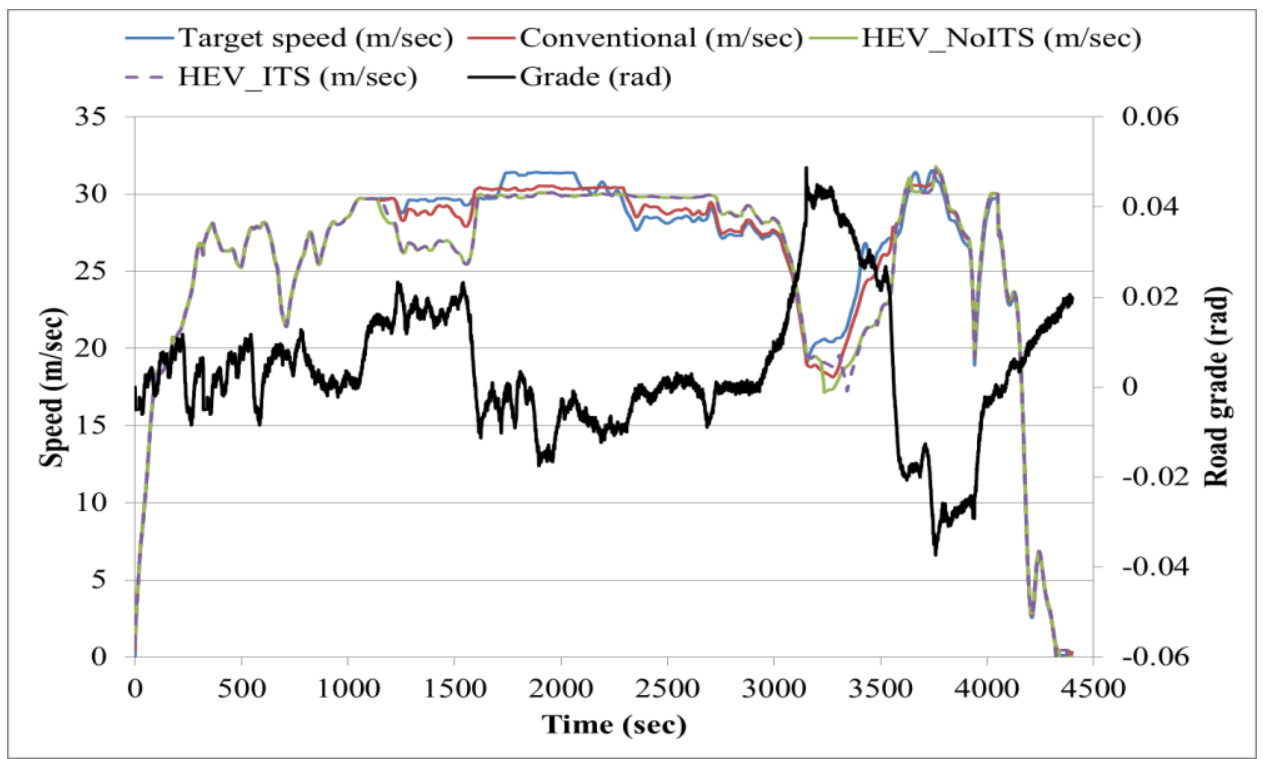

Figure 5.48: Impact of looking-ahead control strategy on maintaining vehicle (GVWR 36,000 kg) speed on Las2Hall cycle

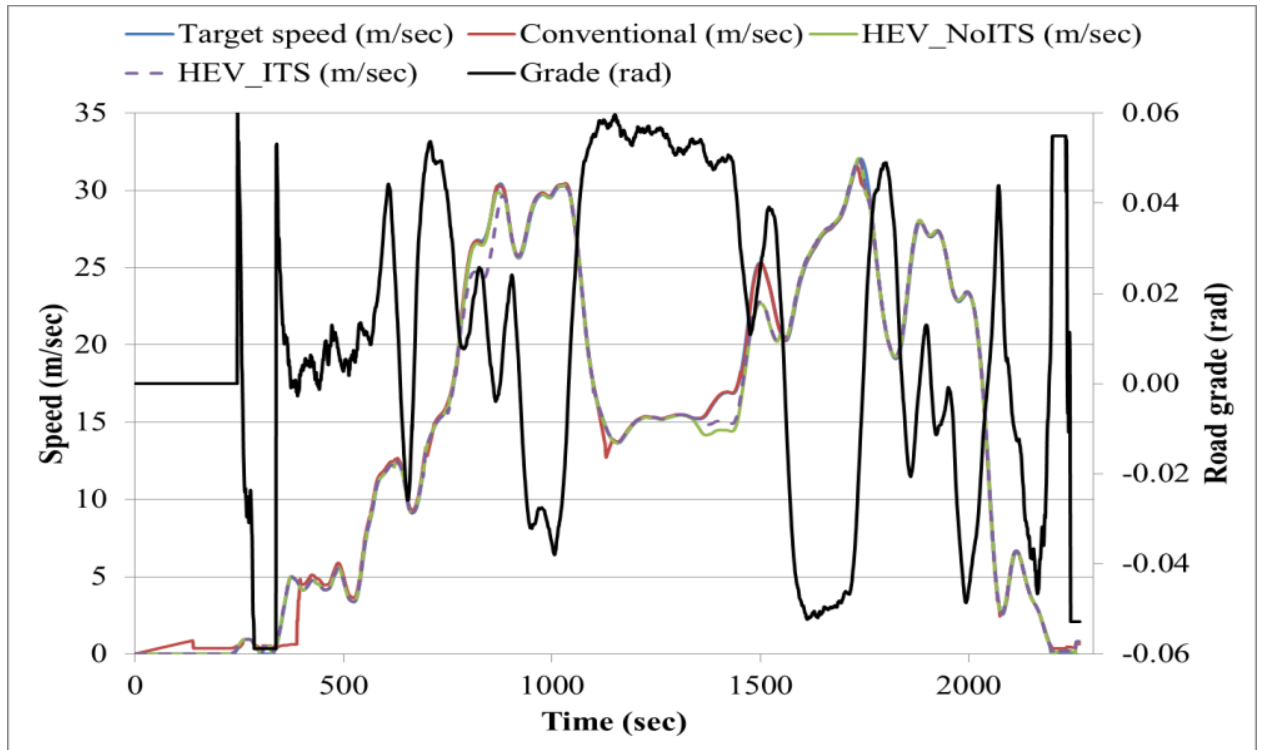

Figure 5.49: Impact of looking-ahead control strategy on maintaining vehicle (GVWR 36,000 kg) speed on Sab2BM cycle 


\subsection{Downsizing engine power opportunity with looking-ahead strategy}

Since the alternative power source is established in HEV, there might be a chance to downsize engine power while maintaining adequate total power for different terrain types. To check the possibility of downsizing the engine power while maintaining same total powertrain performance, different real world driving cycles were used at $100 \%$ full load vehicle model (maximum possible load). The engine size for this model became $310 \mathrm{~kW}$ instead of the original one, which was $410 \mathrm{~kW}$. All other equipment sizes didn't change. The parallel HEV model for this test was $150 \mathrm{~kW}$ and $10 \mathrm{kWh}$ for the electric motor-generator and battery sizes respectively. The scenario of looking-ahead strategy used was Scenario 5, which is looking for practical forthcoming vehicle speed and road grade. The conventional and HEV model were for these results in this section at $100 \%$ full load. Figure (5.50) shows the vehicle speed for a conventional truck model with full size engine compared to the parallel HEV (150 kW and $10 \mathrm{kWh}$ equipment sizes) with a smaller engine $(310 \mathrm{~kW})$ at up-downhill constant speed cycles.

Figures (5.51 and 5.52) show the engine power and electric motor power for the conventional and HEV (with NoITS and ITS) models, and Figure (5.53) shows the battery SOC for the same cycle. Results show that the HEV with looking-ahead strategy can improve the ability to downsize engine power while maintaining adequate total vehicle power for most cycle time with different road grades. Several real cycles based on the distance travelled investigated to show that the parallel HEV model with smaller engine and with ITS can finish at the same point at the same position in comparison to the parallel HEV model with full size engine and without ITS. Looking-ahead time to start preparing the system for forthcoming traffic conditions needs to be increased, and that time increases because of limited extra engine power available to charge the battery preparing to go uphill. For example, the window in the future regarding traffic conditions was about four minutes (based on equipment sizes, see Chapter 3 for more details) ahead for $150 \mathrm{~kW}$ and 10kWh (motor and battery) equipment sizes respectively, but with smaller engine power the time window in the future (for forthcoming traffic conditions) needs to be longer, such as six minutes. For Figure (5.51), the engine power shows different responses at the last 1000 seconds of the trip, and these differences are due to the VPC strategy with and without lookingahead. The parallel HEV model without looking-ahead strategy depleted the energy stored in the 
ESS and sustained the battery SOC at the middle. However, the parallel HEV model didn't deplete the ESS until about to finish the trip. Both strategies ended at the same battery SOC.

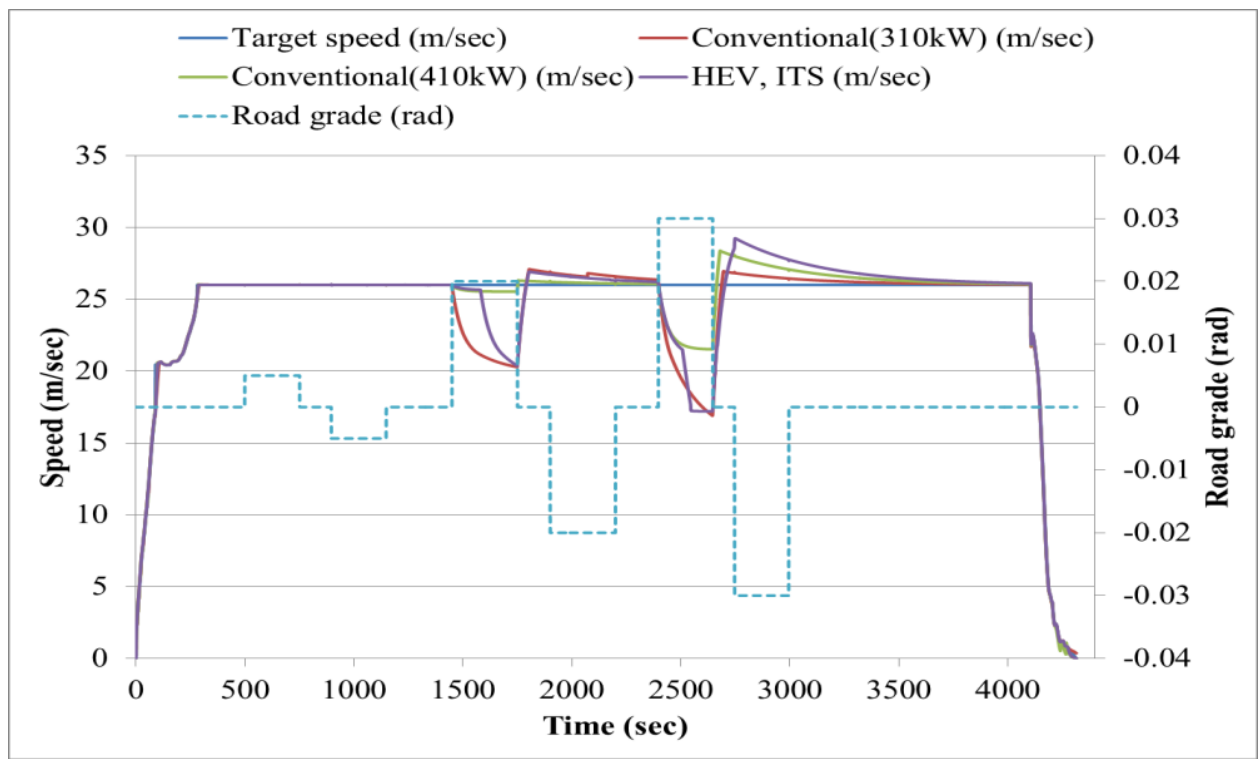

Figure 5.50: Vehicle speed for model (GVWR 36,000 kg) with conventional engine and with smaller engine relative to HEV model with ITS

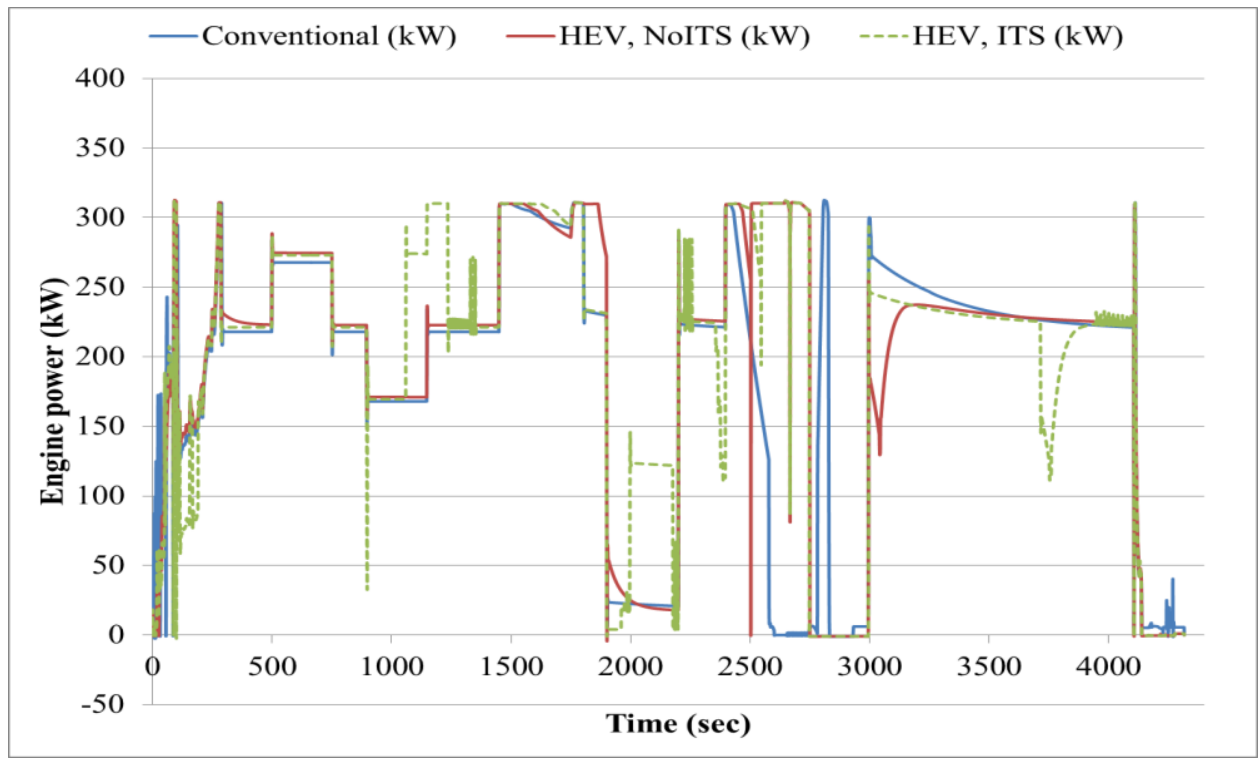

Figure 5.51: Engine power for a conventional (smaller engine) and HEV (GVWR $36000 \mathrm{~kg}$ ) with ITS and No ITS 


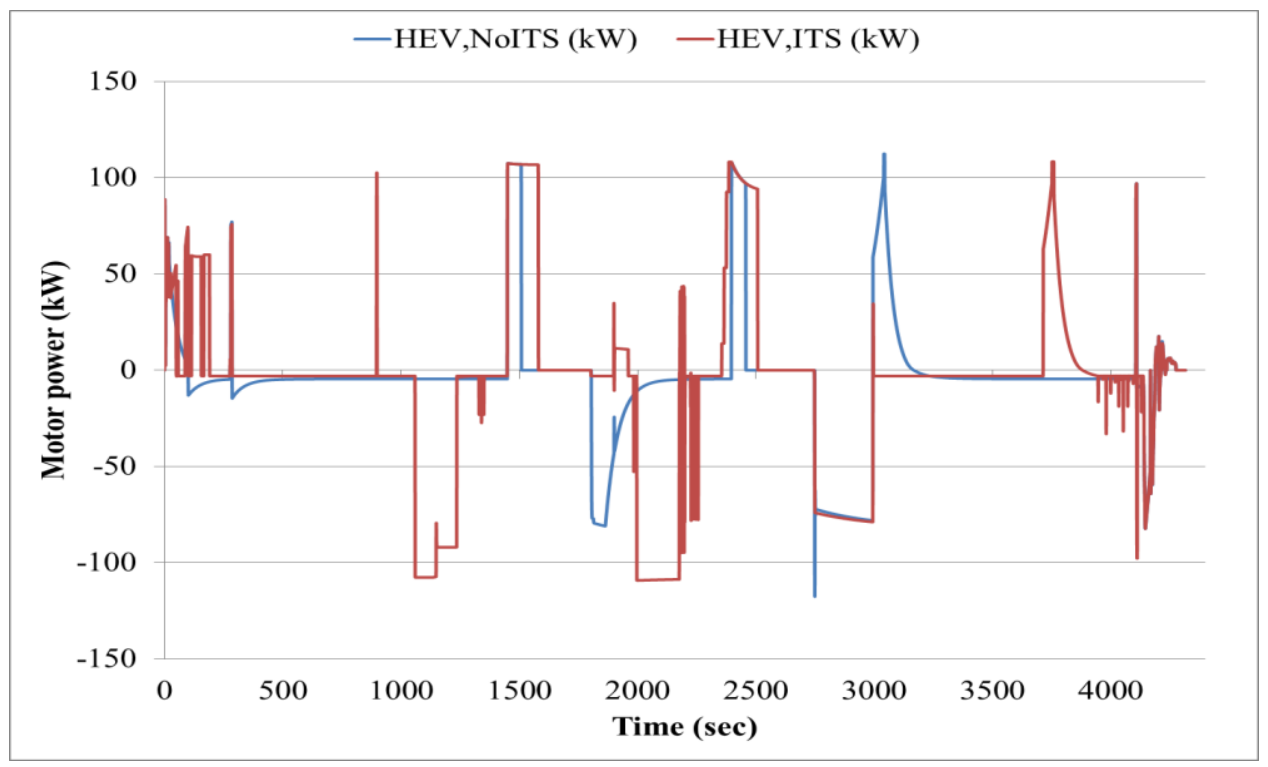

Figure 5.52: Motor power for a conventional (smaller engine) and HEV (GVWR 36,000 kg) with ITS and No ITS

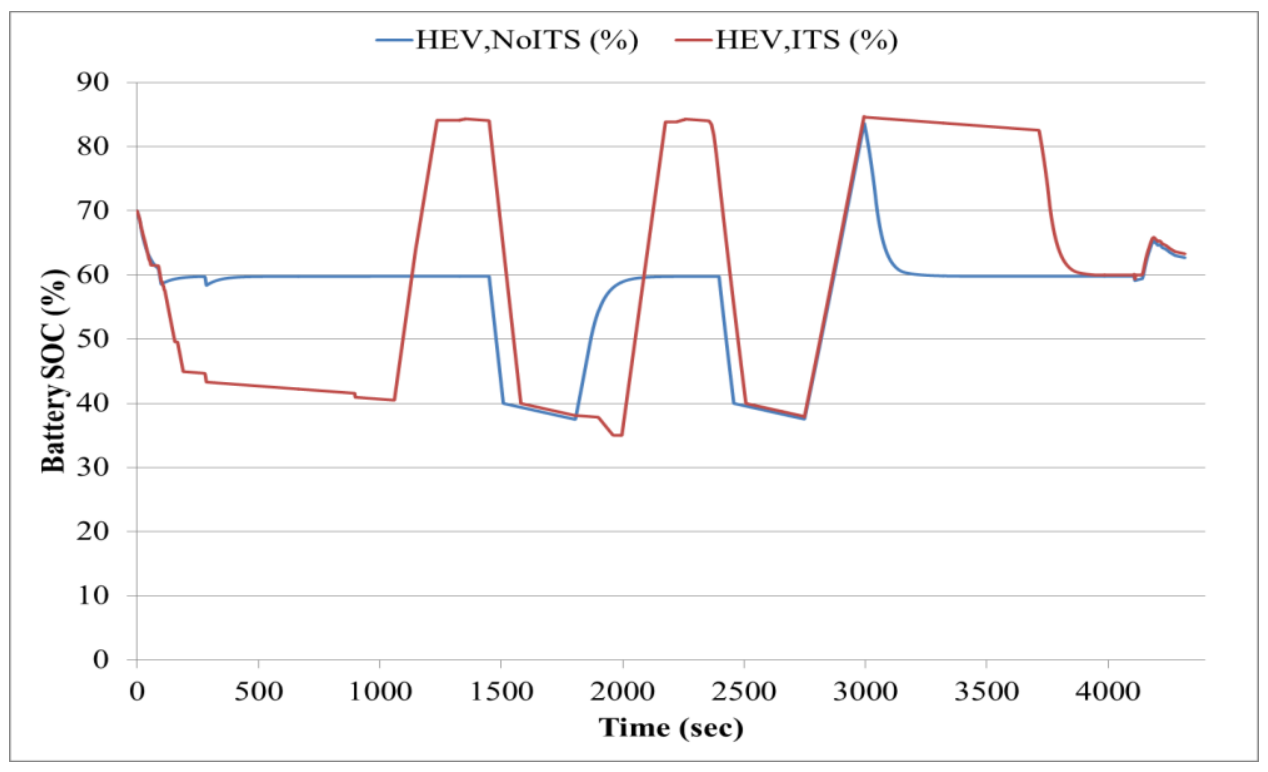

Figure 5.53: Battery SOC for the HEV (GVWR 36,000 kg) with ITS and No ITS 
Looking-ahead strategy shows a benefit on maintaining adequate power, even with downsizing the engine power. Figure (5.54) shows the cumulative fuel consumption verses distance traveled for Sab2BM real world driving cycle. The conventional vehicle model with smaller engine revealed less fuel consumption. However, that decrease of fuel consumption was on account of distance traveled. In other words, the conventional vehicle with smaller engine power didn't reach the destination (due to periods of insufficient engine's power during the trip). That means the engine power was not sufficient during the entire trip. Therefore, the HEV with smaller engine and looking-ahead strategy was the optimum between these three vehicles models types (i.e. conventional with full engine, conventional with smaller engine and HEV with small engine). This result was for $100 \%$ full load model $(36,000 \mathrm{~kg})$ with $150 \mathrm{~kW}$ and $10 \mathrm{kWh}$ equipment sizes.

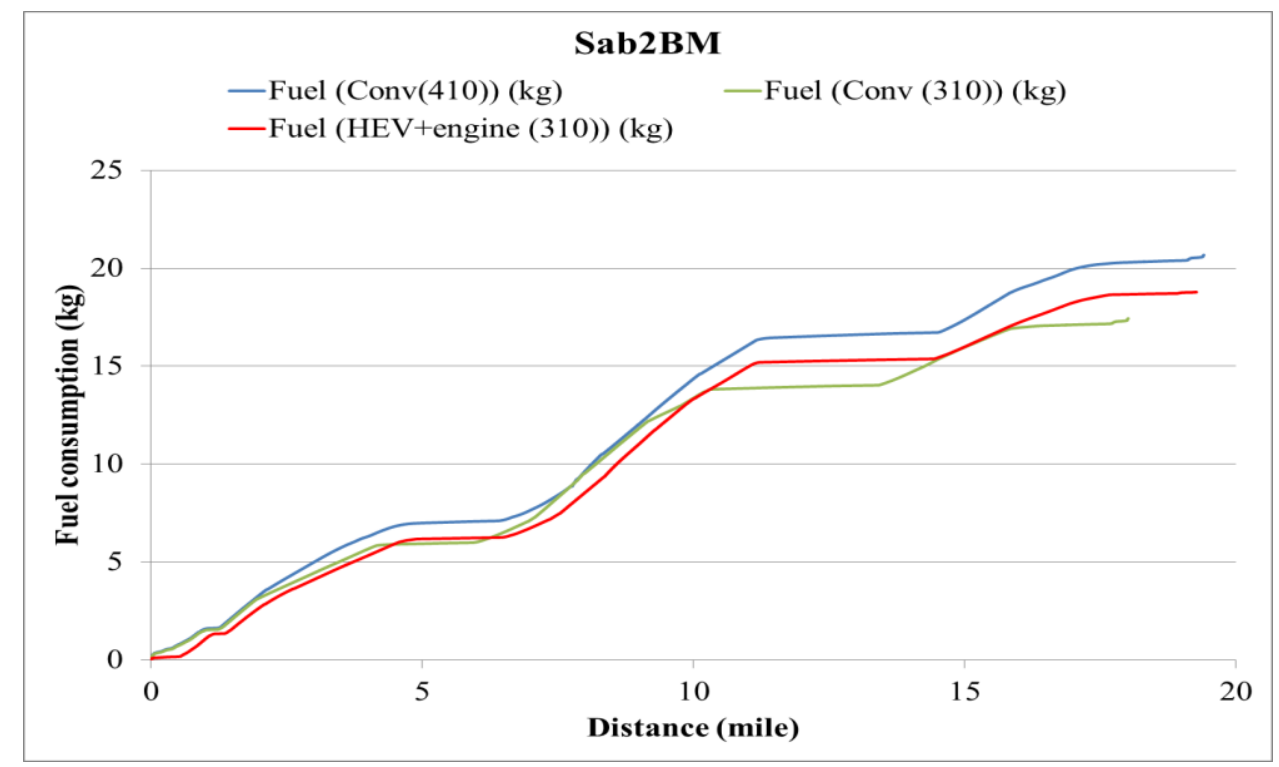

Figure 5.54: Cumulative fuel consumption verses distance traveled for three vehicles modeled on Sab2BM real world driving cycle

To assess the benefit from downsizing engine power, the new HEV model should finish the same route finished by the conventional model with full size engine power (410). Figures (5.55 and 5.56) revealed that both the HEV model with the smaller engine $(310 \mathrm{~kW})$ and looking-ahead strategy and the model with the conventional full size engine $(410 \mathrm{~kW})$ accomplished almost same route, about $99 \%$ of the route distance. However, the conventional model with smaller engine $(310 \mathrm{~kW})$ didn't finish the route at the same time, which means that this model can't be 
compared to the other models in fuel economy perspective (apple to apple point of view). These results were for constant speed with different road grades and real world driving cycle Sab2BM respectively. The other important finding from this work is that the HEV model with smaller engine may become worse on longer ascending hills because batteries get depleted.

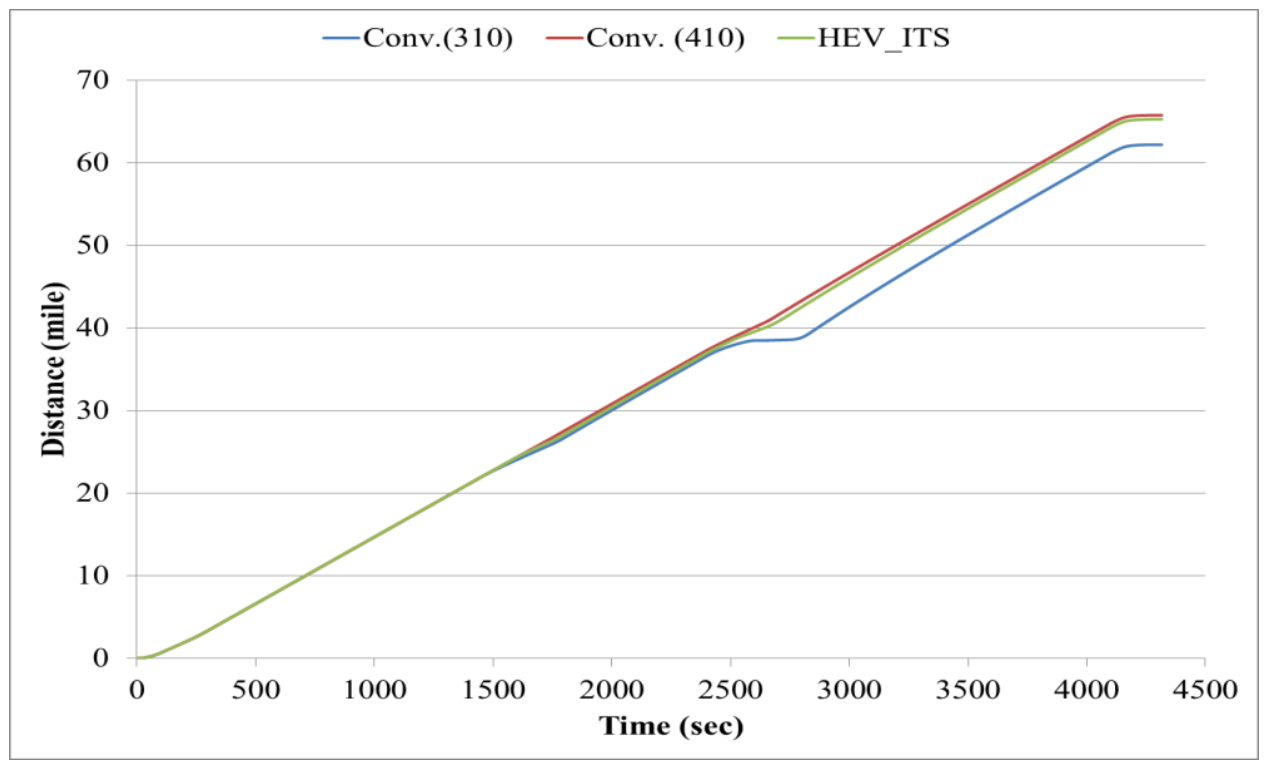

Figure 5.55: Distance finished by different models on constant speed with different road grade route

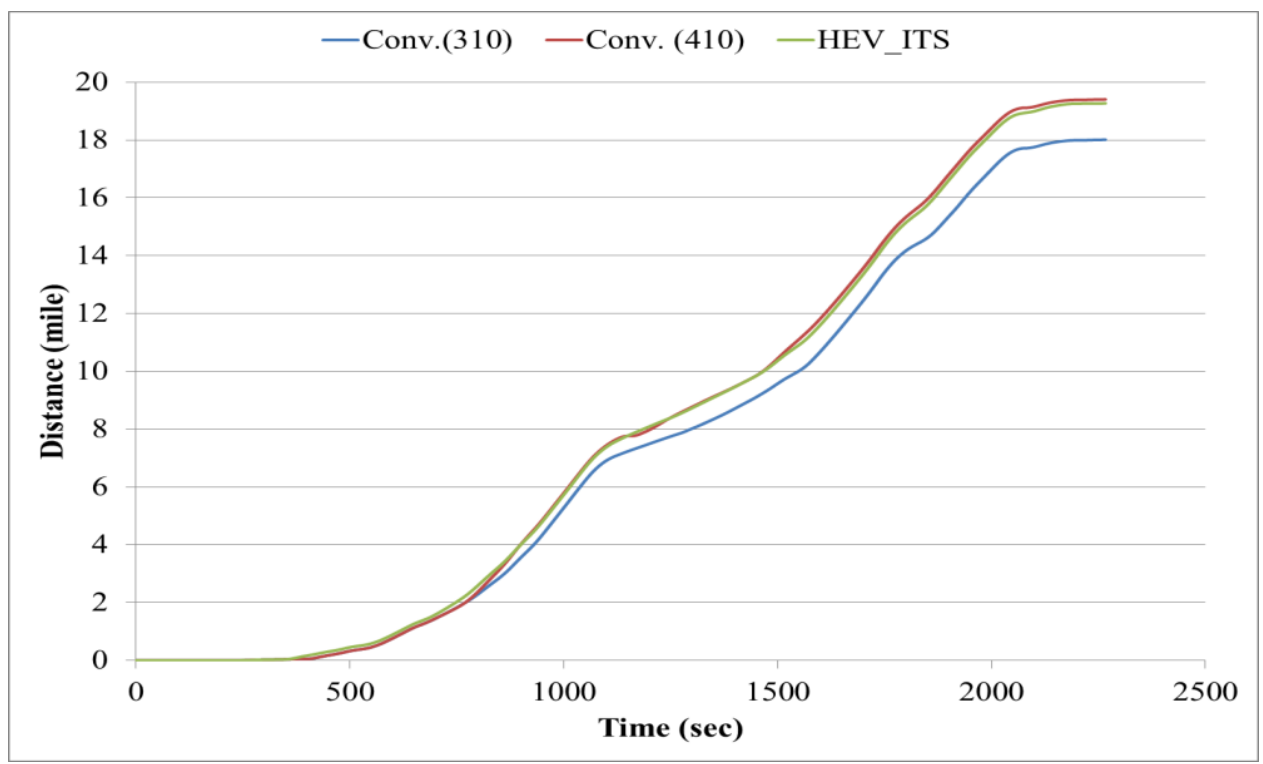

Figure 5.56: Distance finished by different models on real world driving cycle Sab2BM route 
Figures (5.57-5.61) show the vehicle speed for a conventional truck model with full size engine in comparison to parallel HEV (150 kW and $10 \mathrm{kWh}$ equipment sizes with smaller engine (310 $\mathrm{kW})$ ) for different real world driving cycles. The vehicle speed for the HEV model with smaller engine wasn't able to follow the target speed all time, but it did it most time.

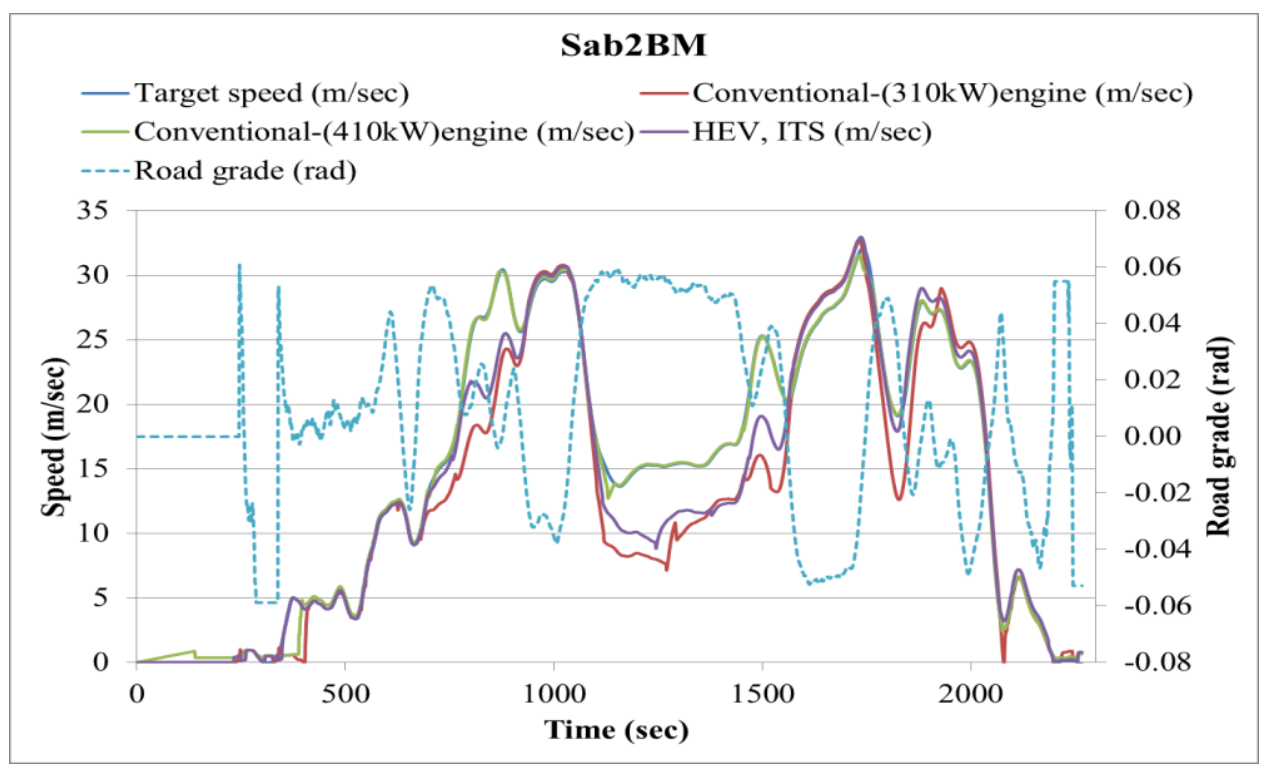

Figure 5.57: Vehicle speed variation due to engine downsize for vehicle model $(\mathrm{GVWR}=36,000 \mathrm{~kg})$ on Sab2BM route

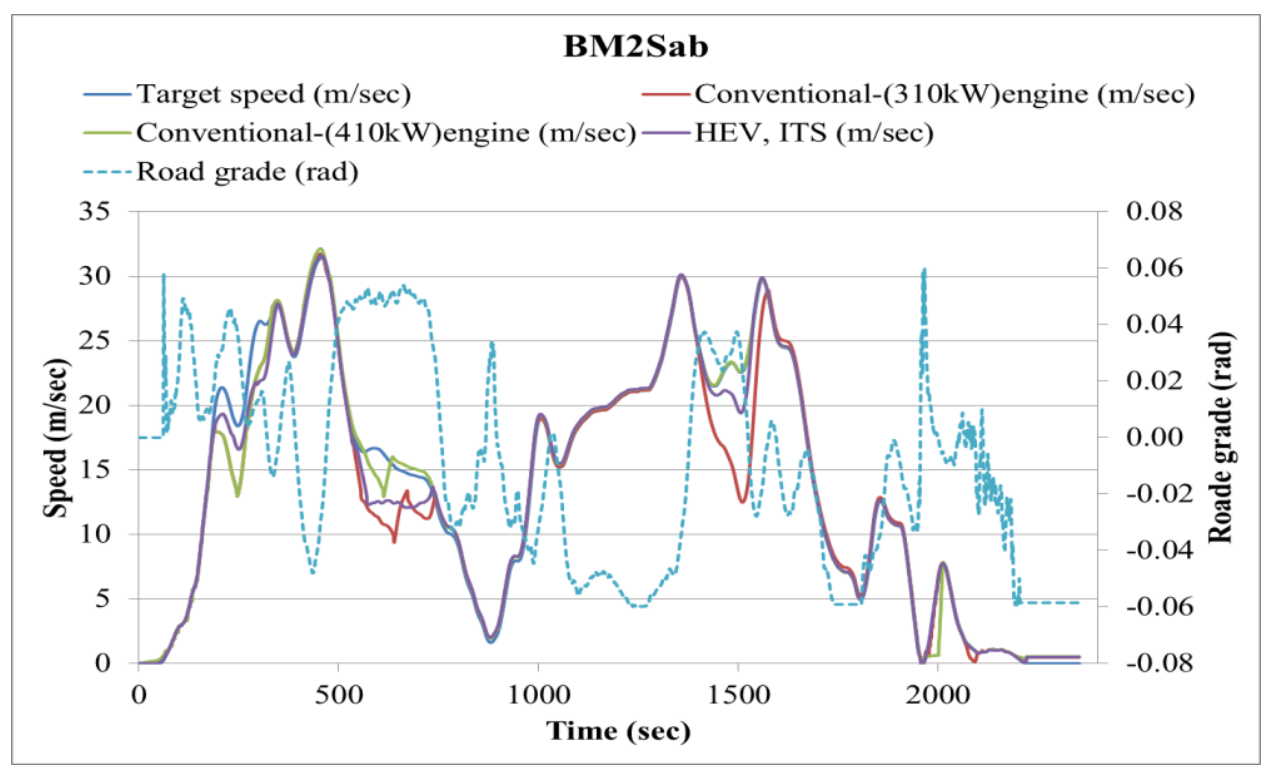

Figure 5.58: Vehicle speed variation due to engine downsize for vehicle model $(\mathrm{GVWR}=36,000 \mathrm{~kg})$ on $\mathrm{BM} 2 \mathrm{Sab}$ route 


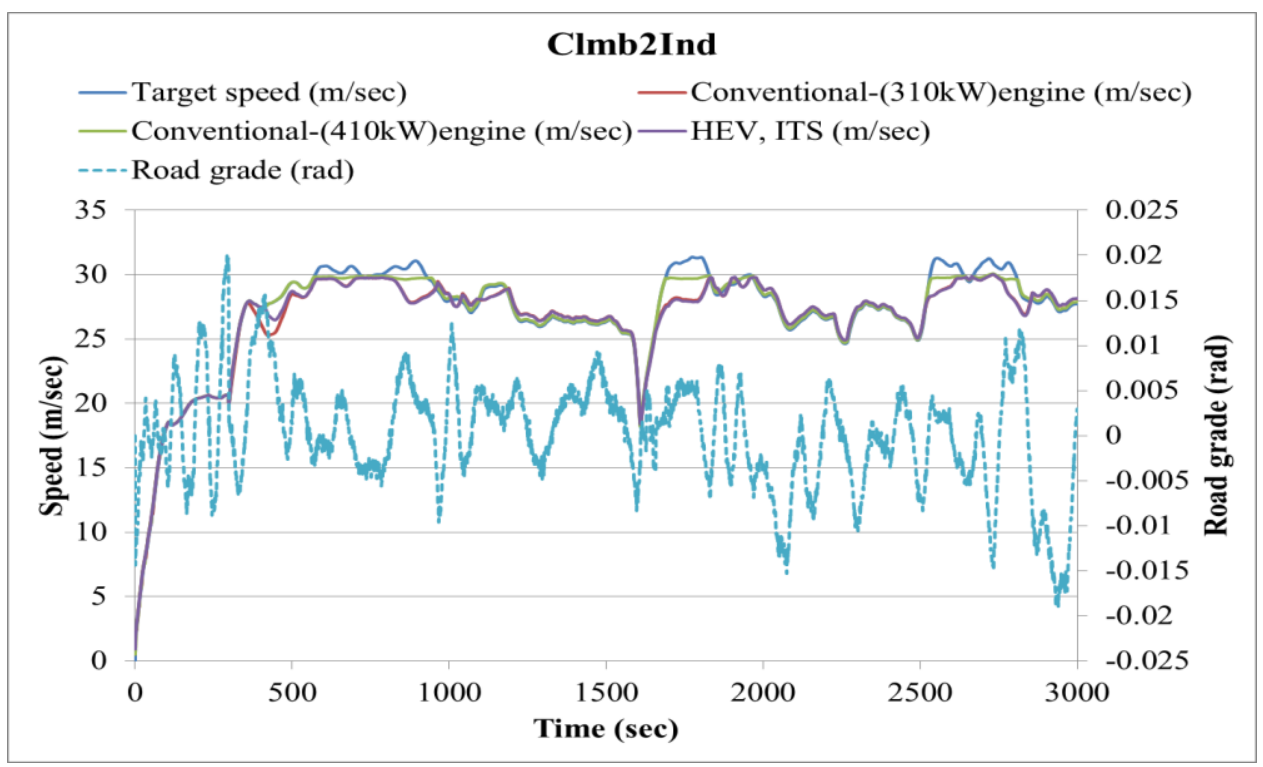

Figure 5.59: Vehicle speed variation due to engine downsize for vehicle model $(\mathrm{GVWR}=36,000 \mathrm{~kg})$ on Clmb2Ind route

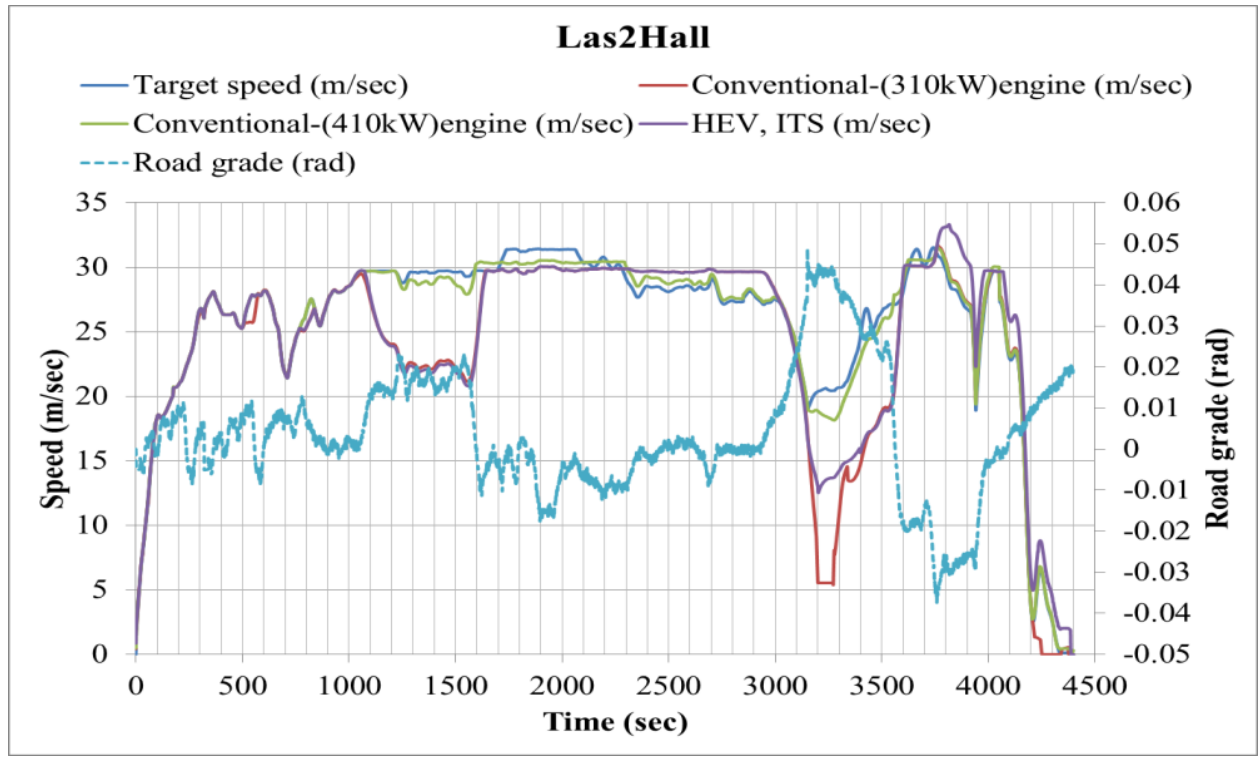

Figure 5.60: Vehicle speed variation due to engine downsize for vehicle model $(\mathrm{GVWR}=36,000 \mathrm{~kg})$ on Las $2 \mathrm{Hall}$ route 


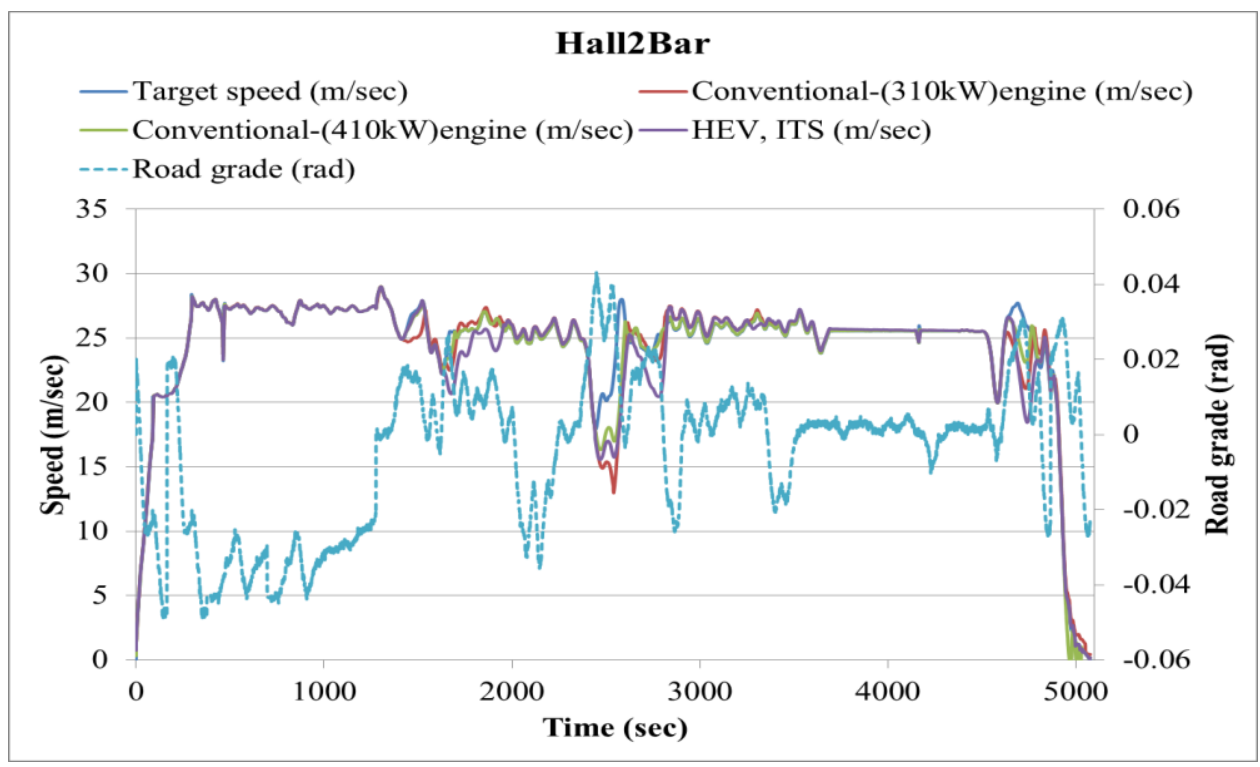

Figure 5.61: Vehicle speed variation due to engine downsize for vehicle model $(\mathrm{GVWR}=36,000 \mathrm{~kg})$ on Hall2Bar route

Figures (5.62-5.67) show the impact of engine power downsizing on the engine efficiency for different real world driving cycles. In other words, the engine downsizing can contribute to improving the engine efficiency by running it at an increased efficiency on the torque-speed map. In the one case, the average efficiency of the engine over the cycle, found in BM2Sab way, was 38\%, whereas the smaller engine with hybrid operation offered higher efficiency, at $39 \%$. Moreover, Figures (5.68 and 5.69) show the impact of engine power downsizing on the alternative power source, the ESS, activity. The ESS activity for smaller engine HEV model was obviously more in comparison to the ESS activity for the original HEV model; the reason for that is that the HEV model with smaller engine needed alternative power source to assist the main power source more frequently. However, using battery more frequently has a penalty due to charging and discharging efficiencies losses. The major challenge of the engine power downsizing is the maintaining of adequate power during different vehicle activities. Therefore, the alternative power source has proven that it is qualified to substitute the power lost due to engine power downsizing in different vehicle activities such as ascending hills, descending hills and flat highway terrains with full load test $(36,000 \mathrm{~kg})$. 


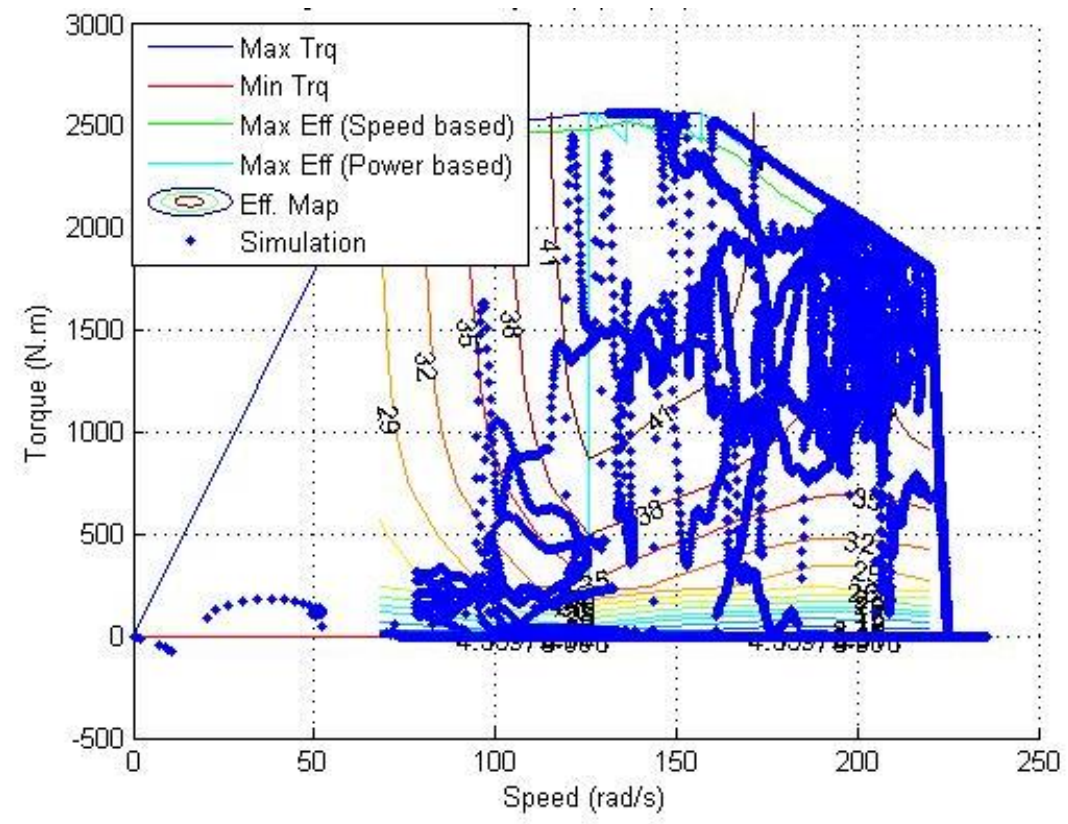

Figure 5.62: Engine hot efficiency map for parallel HEV model with full size engine $(410 \mathrm{~kW})$ at Las 2 Hall driving cycle

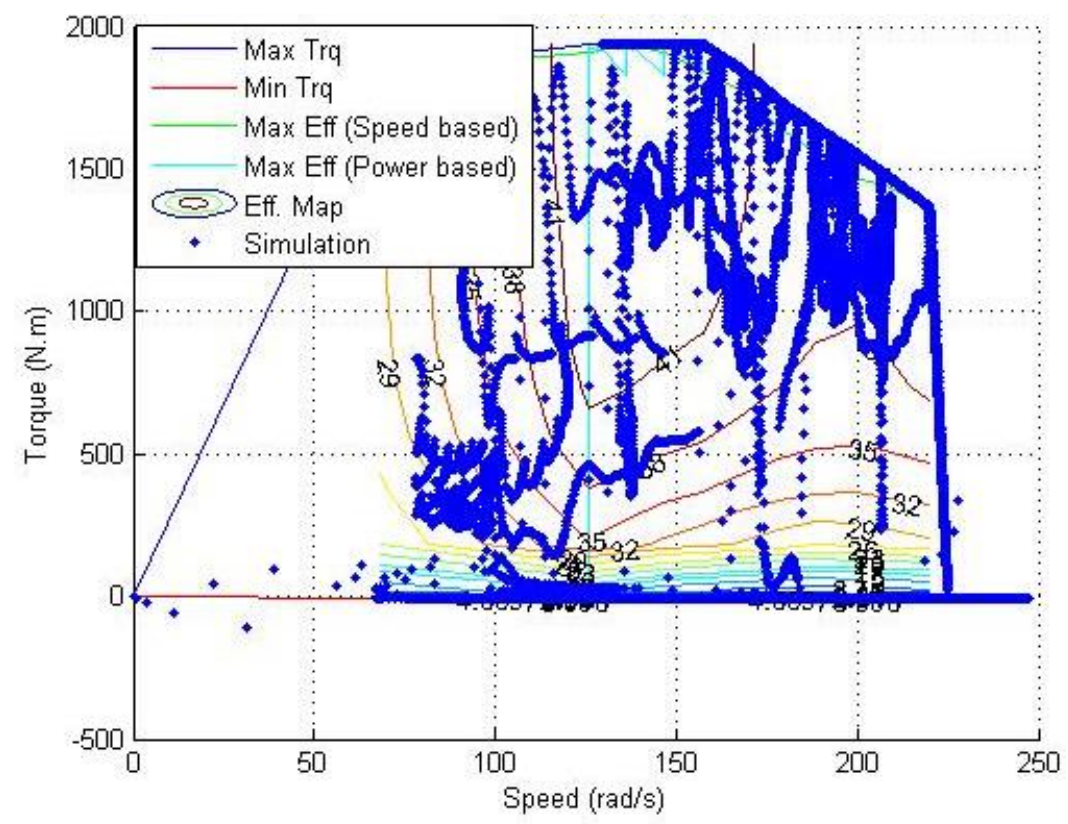

Figure 5.63: Engine hot efficiency map for parallel HEV model with smaller engine size $(310 \mathrm{~kW})$ at Las 2 Hall driving cycle 


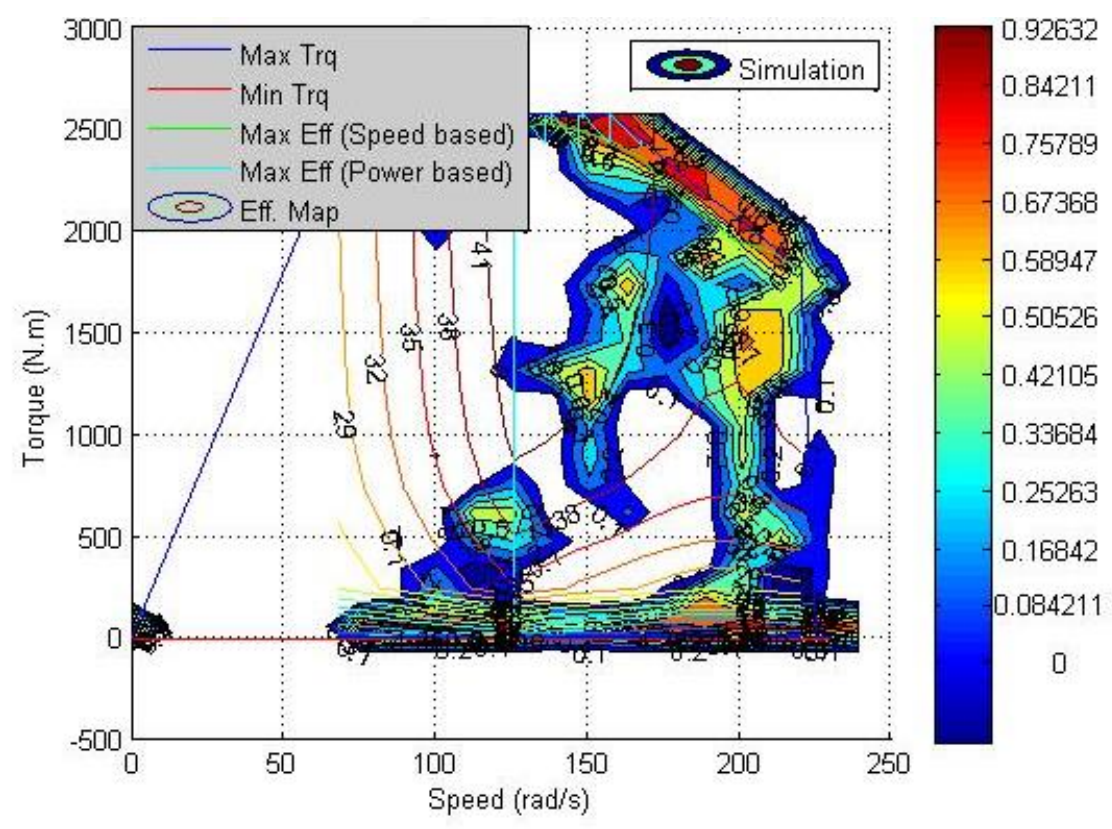

Figure 5.64: Engine hot efficiency map for parallel HEV model with full engine size $(410 \mathrm{~kW})$ at Sab2BM driving cycle

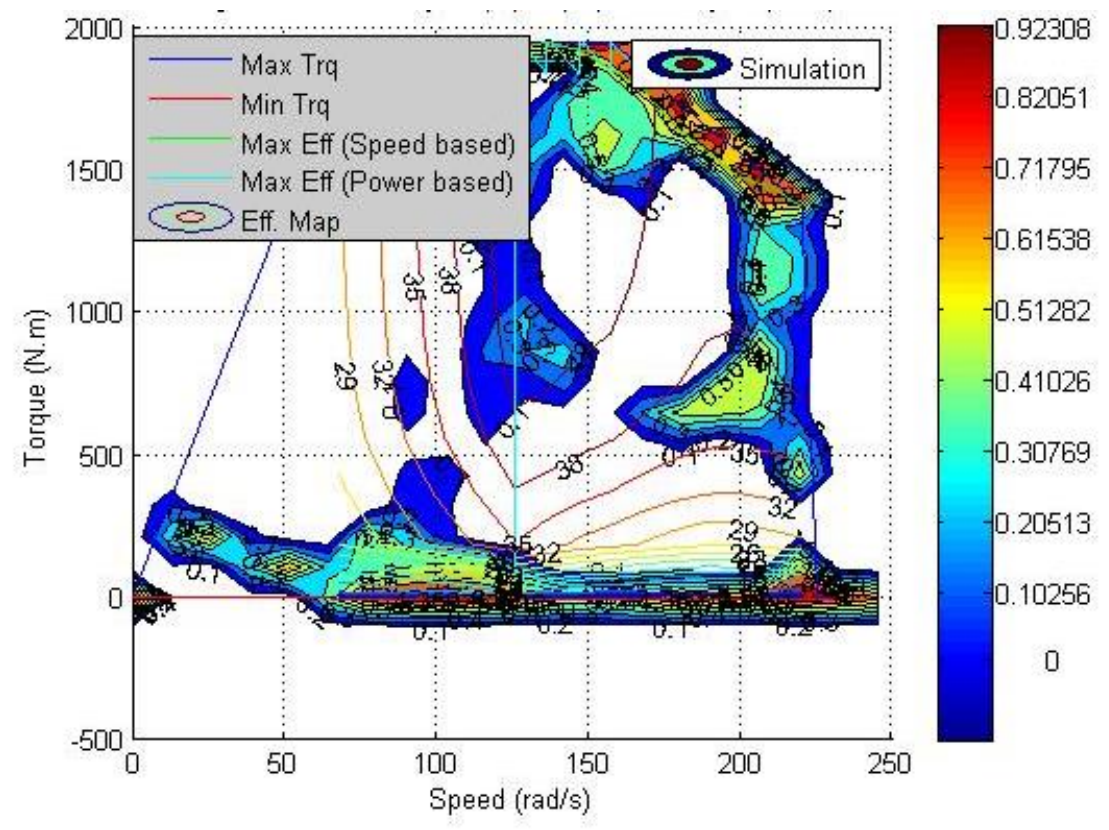

Figure 5.65: Engine hot efficiency map for parallel HEV model with smaller engine size $(310 \mathrm{~kW})$ at Sab2BM driving cycle 


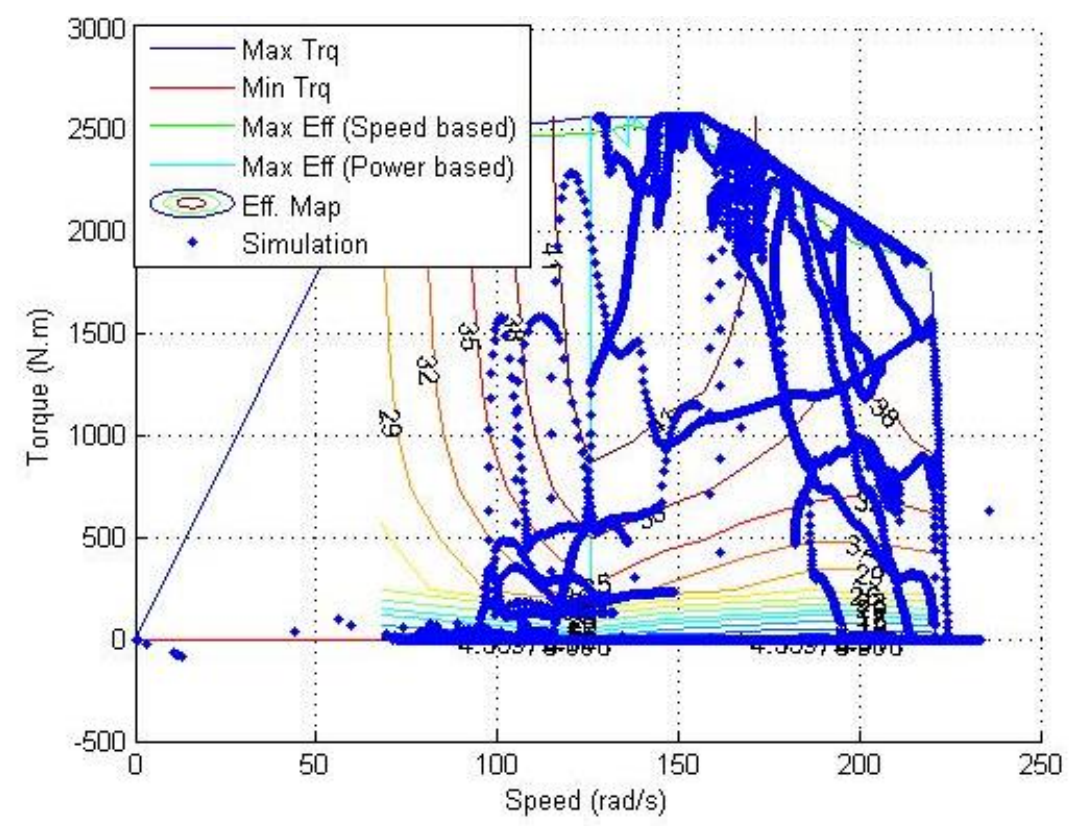

Figure 5.66: Engine hot efficiency map for parallel HEV model with full size engine $(410 \mathrm{~kW})$ at BM2Sab driving cycle

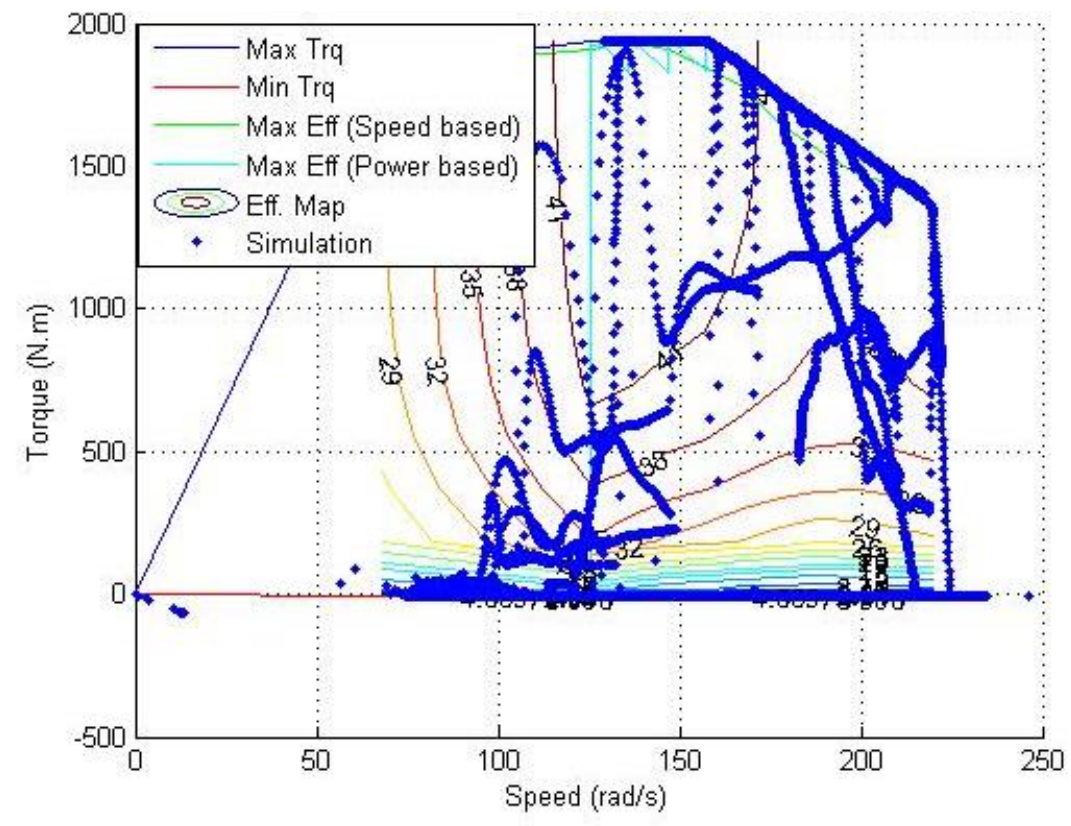

Figure 5.67: Engine hot efficiency map for parallel HEV model with smaller size engine $(310 \mathrm{~kW})$ at BM2Sab driving cycle 


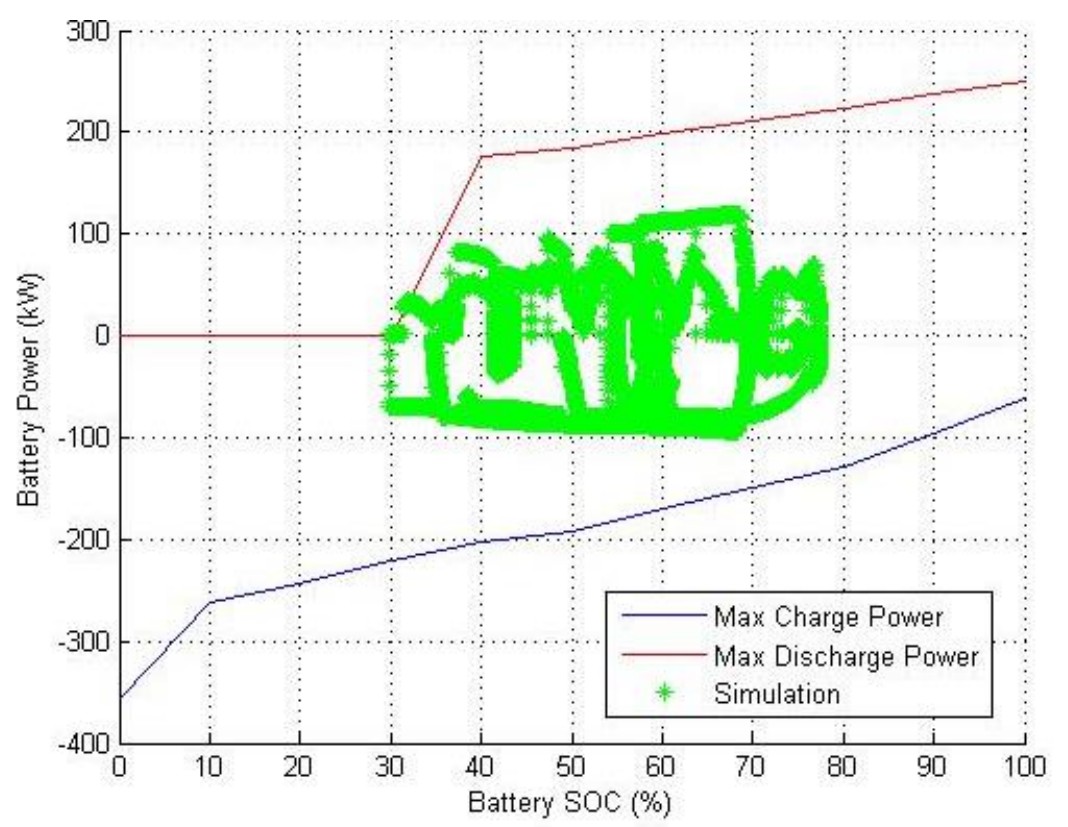

Figure 5.68: ESS activity on Sab2BM driving cycle with full engine size $(410 \mathrm{~kW})$

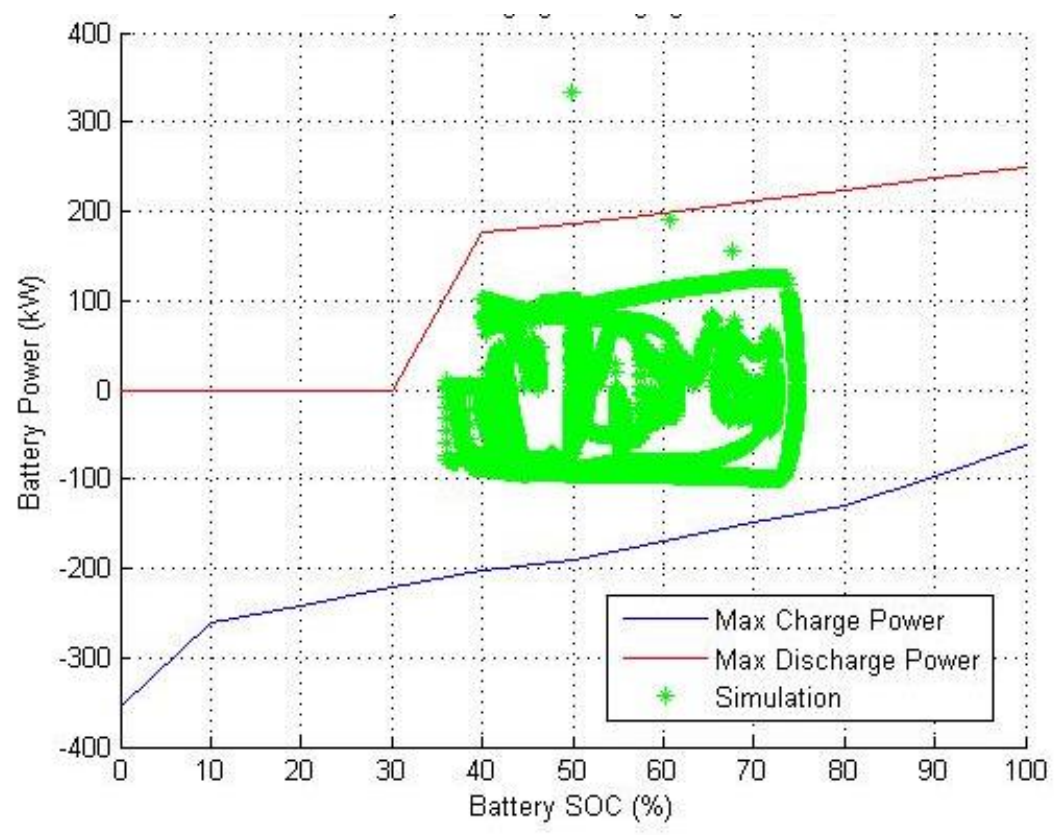

Figure 5.69: ESS activity on Sab2BM driving cycle with smaller engine size $(310 \mathrm{~kW})$ 
Figures (5.70 and 5.71) show the fuel economy and fuel economy change due to downsizing the engine power for a HEV with looking-ahead control strategy for full size engine and smaller engine models. These results show that the downsizing the engine with looking-ahead control strategy is possible and it also can maintain almost the same powertrain performance. Engine efficiency can improve due to downsizing engine power.

Figures (5.72 and 5.73) show the impact of downsizing engine power on engine efficiency for real world driving cycles. The results show that the fuel economy could be improved by as much as $13 \%$ with downsizing engine power by $100 \mathrm{~kW}$, and these results are for parallel HEV model with looking-ahead strategy.

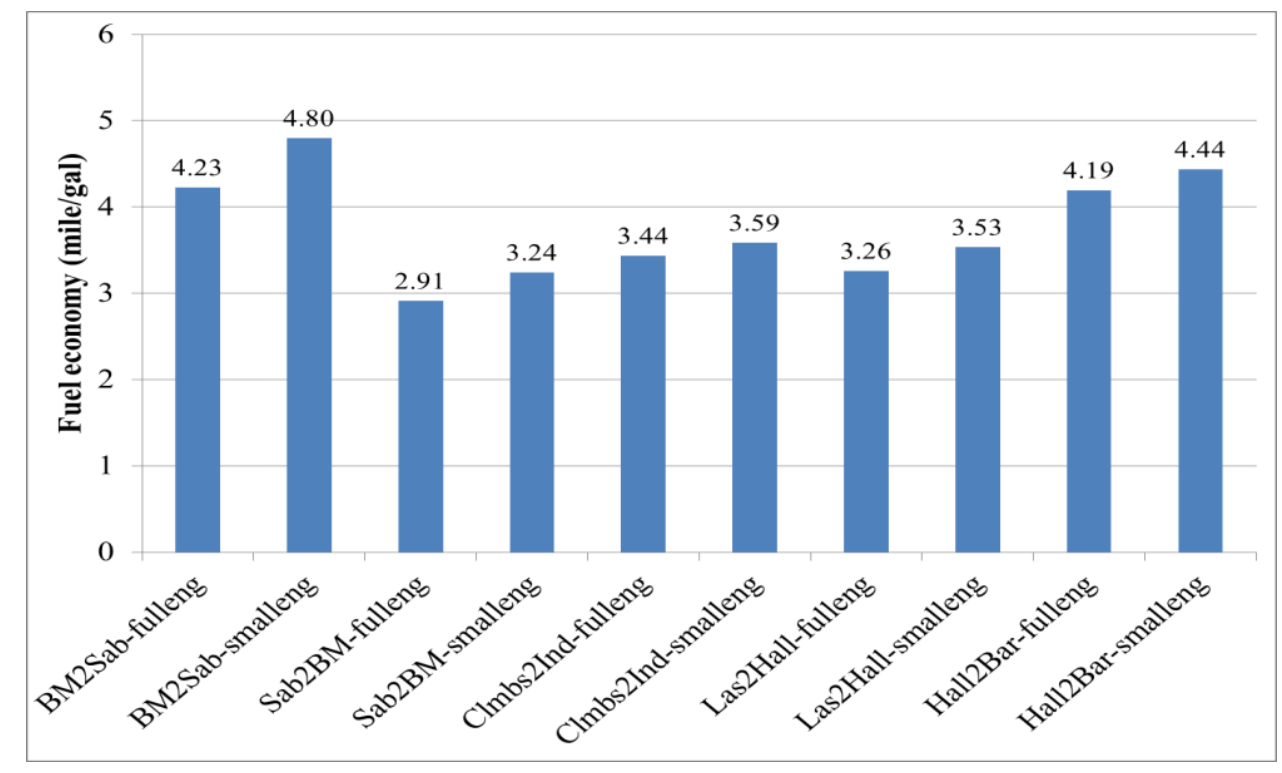

Figure 5.70: Fuel economy for parallel HEV_150kW_10kWh_ITS model $(\mathrm{GVWR}=36,000 \mathrm{~kg})$ with full size engine and smaller engine with looking-ahead control strategy 


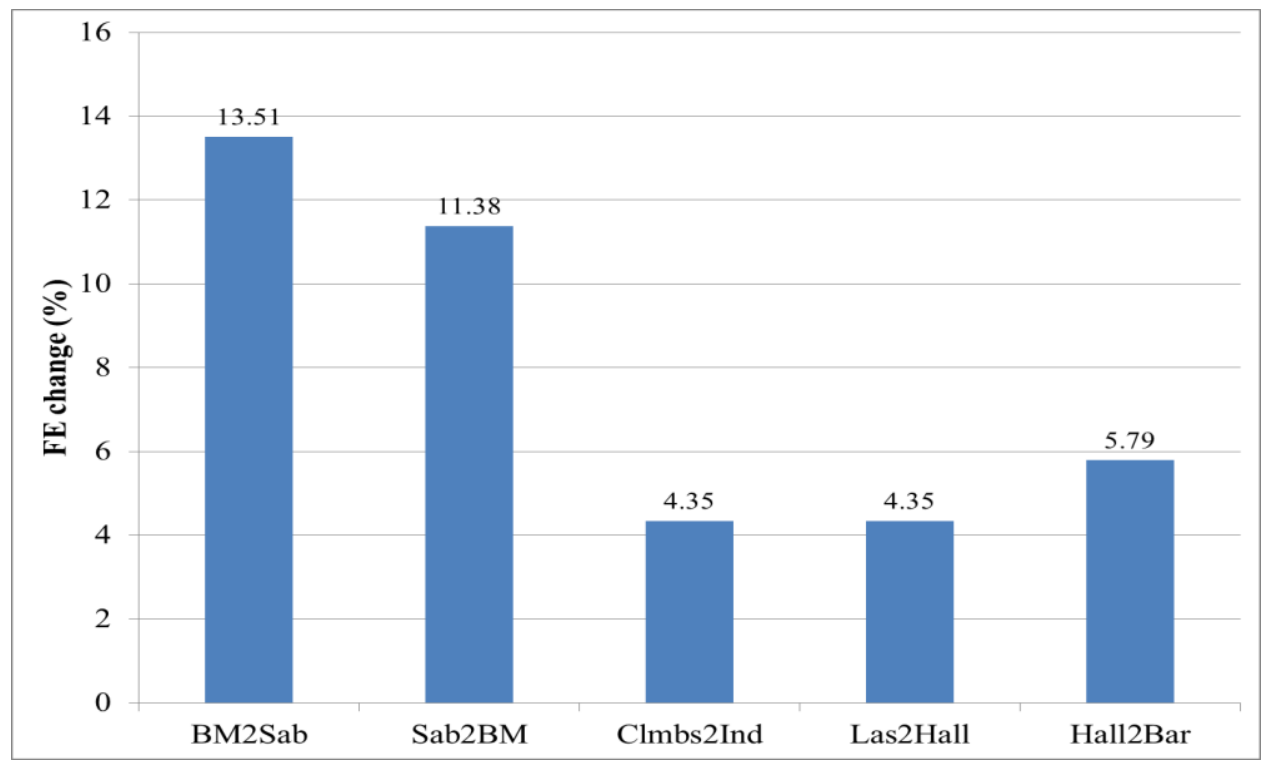

Figure 5.71: FE change for parallel HEV_150kW_10kWh_ITS model $(\mathrm{GVWR}=36,000 \mathrm{~kg})$ with full size engine and smaller engine with looking-ahead control strategy

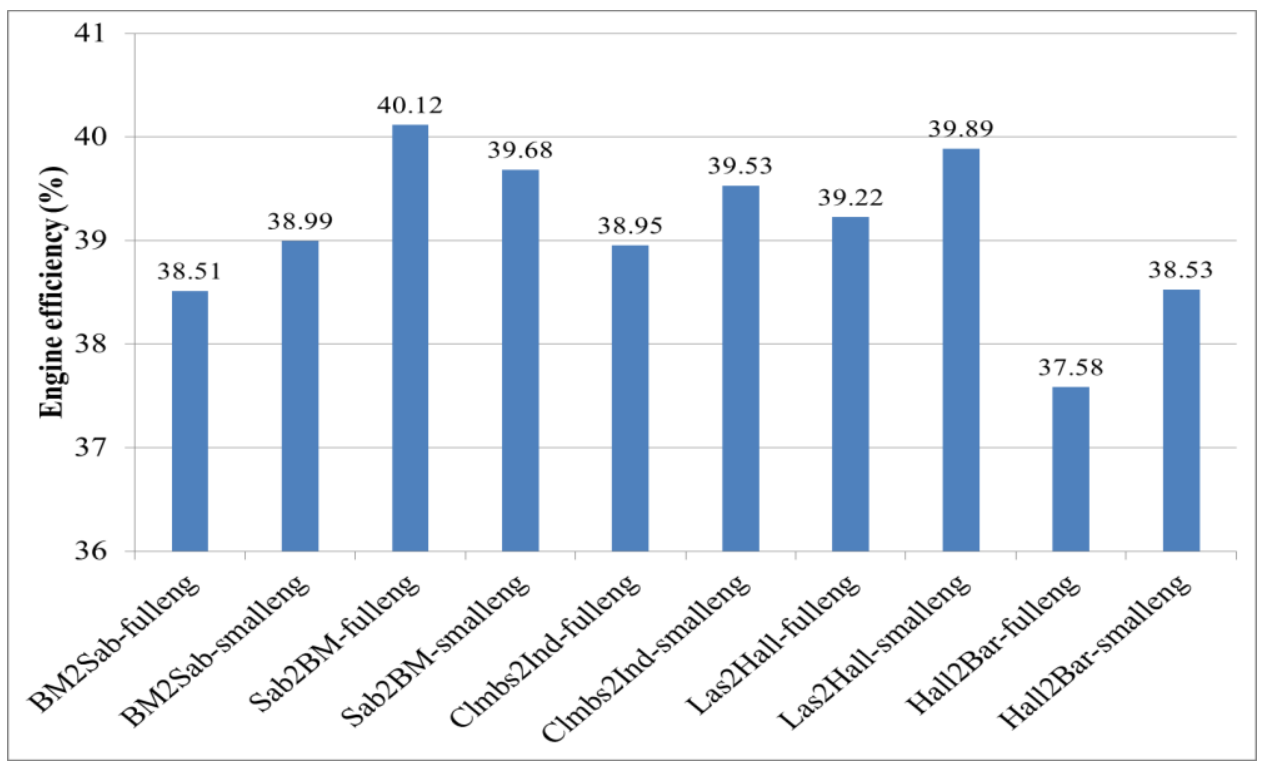

Figure 5.72: Engine efficiency for parallel HEV_150kW_10kWh_ITS model (GVWR $=36,000 \mathrm{~kg})$ with full size engine and smaller engine with looking-ahead control strategy 


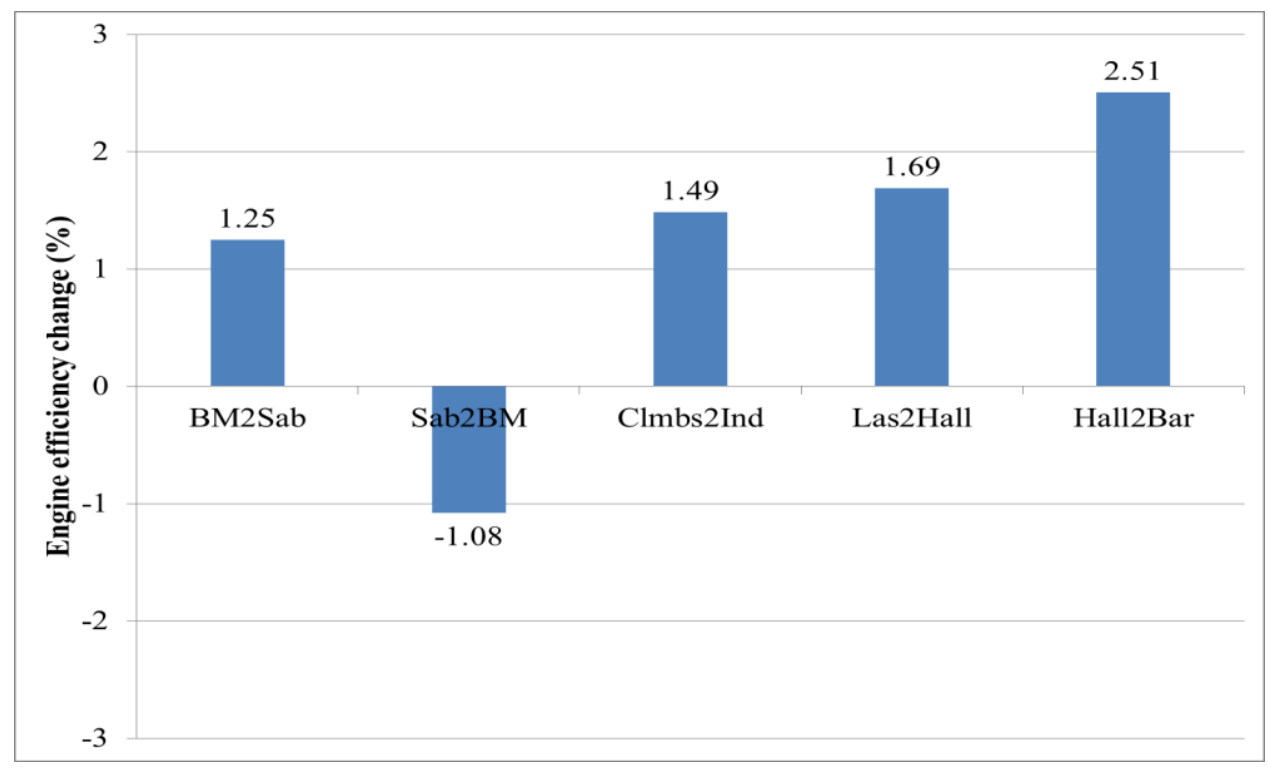

Figure 5.73: Engine efficiency change for parallel

HEV_150kW_10kWh_ITS model $(\mathrm{GVWR}=36,000 \mathrm{~kg})$ with full size

engine and smaller engine with looking-ahead control strategy

\subsection{Simulation results goodness for different driving cycles}

The main purposes of any improvement on vehicle powertrain system are to improve fuel economy and to maintain adequate power (target vehicle speed) during different vehicle activities and on different terrain types. The square of correlation coefficients $\left(\mathrm{R}^{2}\right)$, the root mean square error (RMSE), and relative error have been used to evaluate the results.

Figures (5.74-5.77) show a sample of linear regression, and there are more figures in the appendix A for more details. The main source for mismatch between the simulated and target vehicle speed is due to insufficient power. Where the plots show operating points below the parity line, the truck with the smaller engine was unable to deliver sufficient power to overcome the road load demands at the target speed. However, this mismatch did not impact the overall HEV model evaluation. Moreover, the HEV models with smaller engine was not able to follow all target speeds during the trip, but the HEV was still able to finish the trip almost at same time (99\% of the route finished) in comparison to HEV model with full size engine. Table (5.1) lists the evaluations of different real world cycles' goodness. 


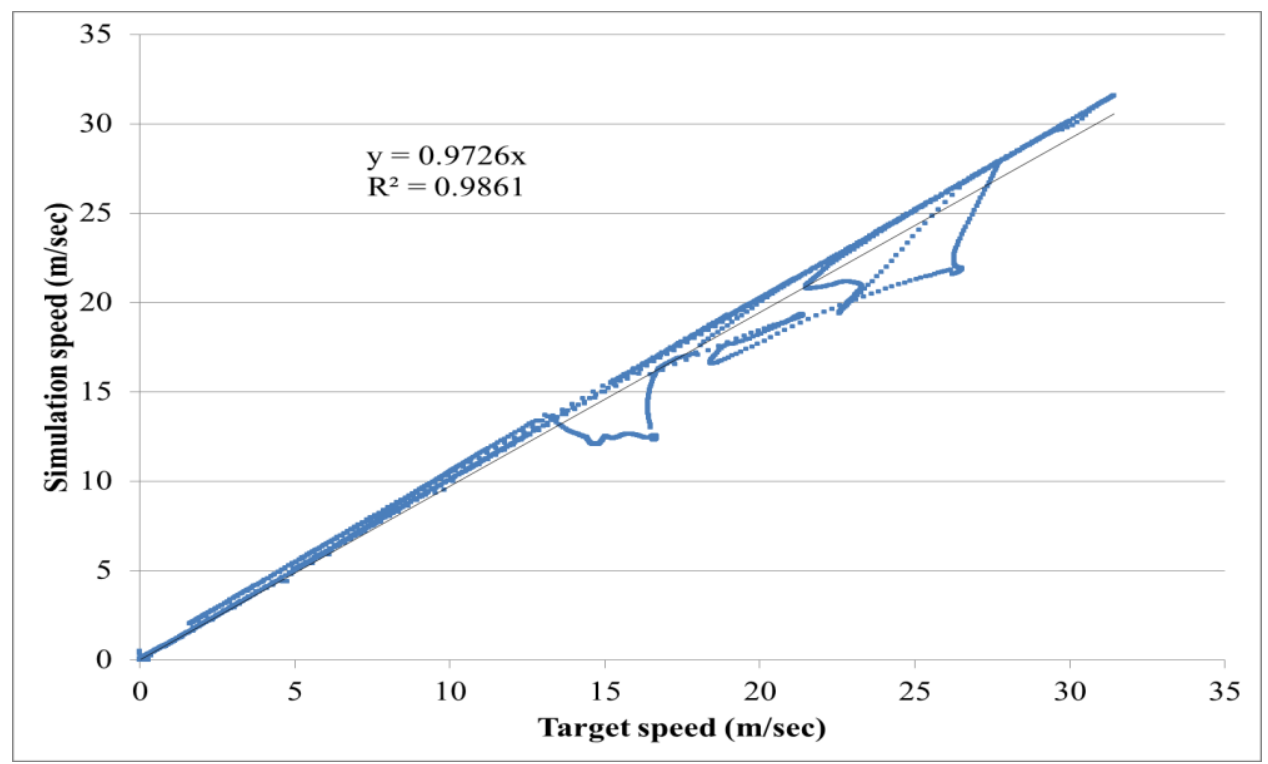

Figure 5.74: Linear regression between simulated HEV with lookingahead and target vehicle speed for BM2Sab driving cycle

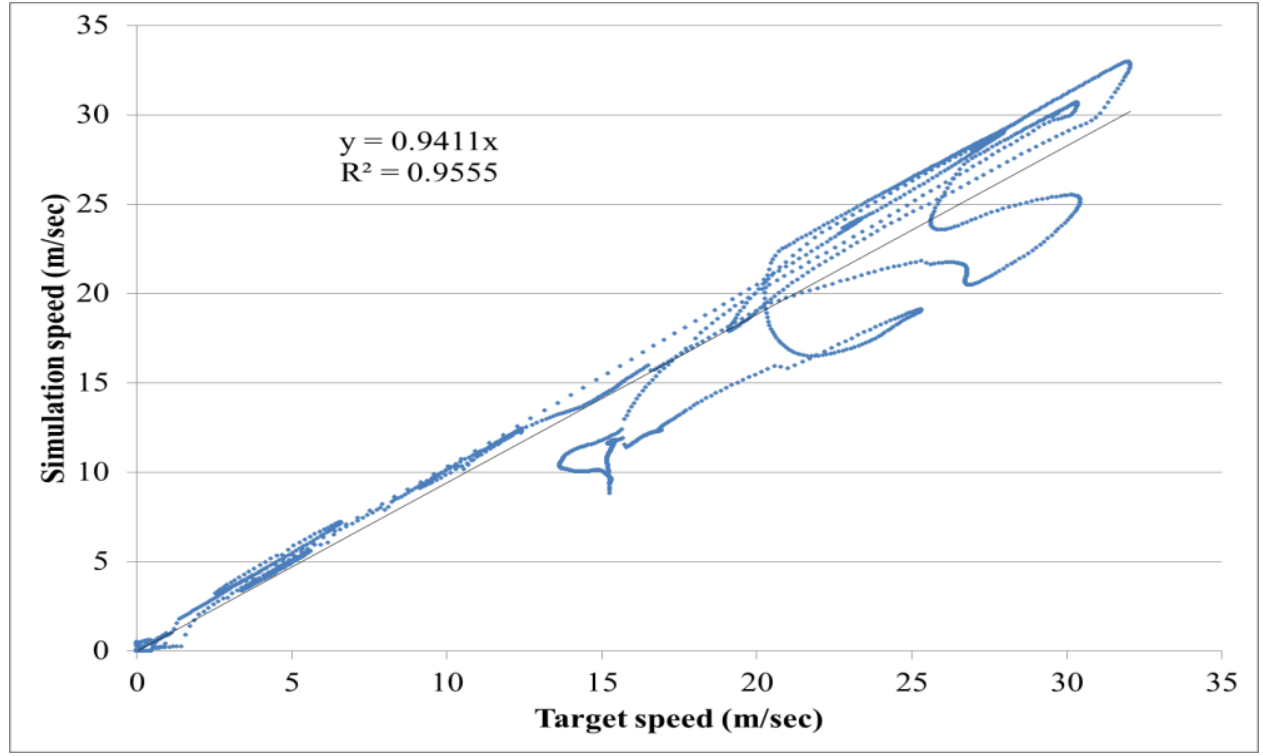

Figure 5.75: Linear regression between simulated HEV with lookingahead and target vehicle speed for Sab2BM driving cycle 


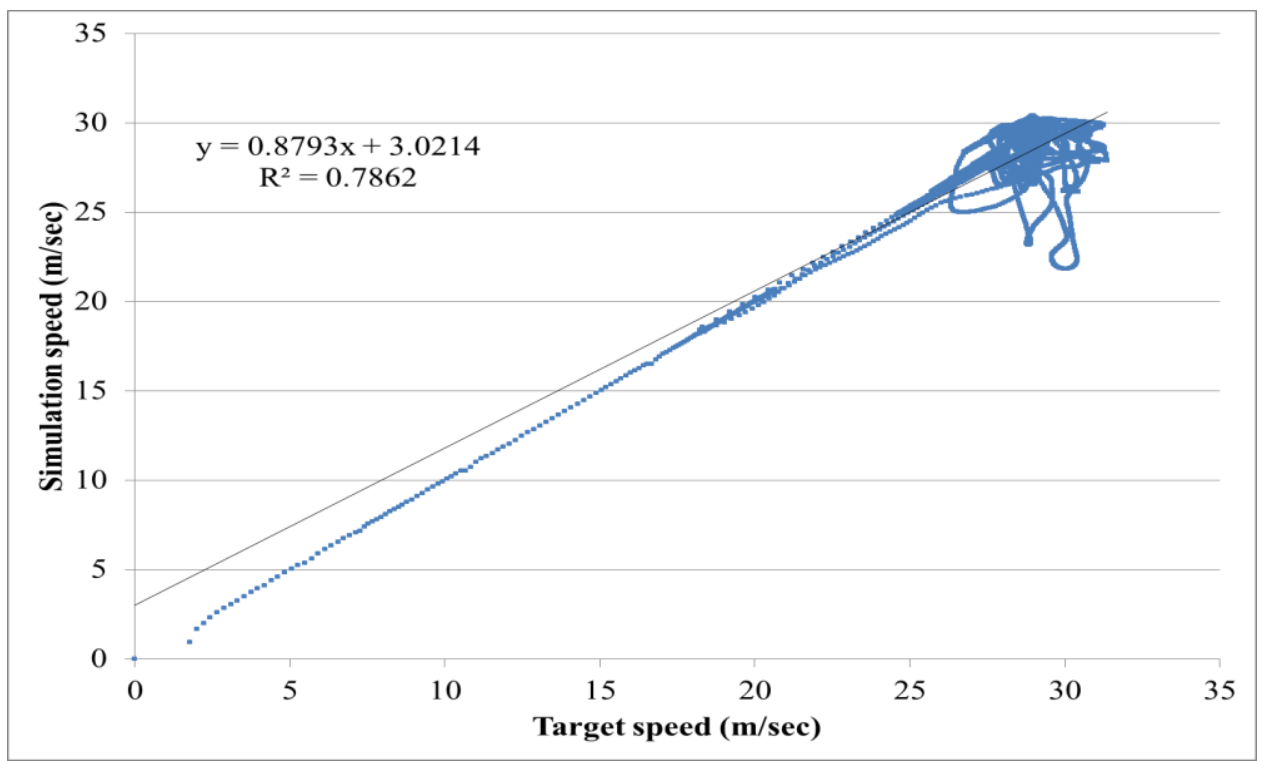

Figure 5.76: Linear regression between simulated HEV with lookingahead and target vehicle speed for CLMB2IND driving cycle

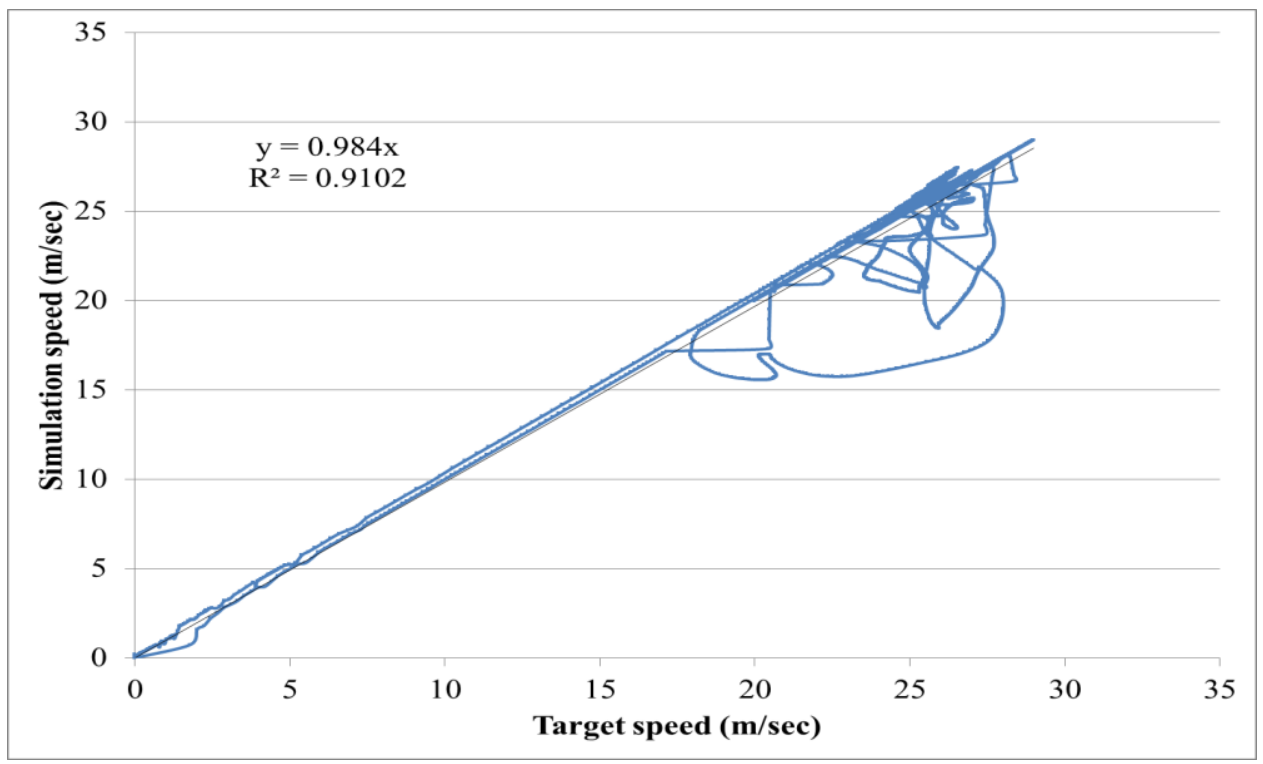

Figure 5.77: Linear regression between simulated HEV with lookingahead and target vehicle speed for Hall2Bar driving cycle 
Table (5.1) shows the accuracy of simulated vehicle speed in comparison with vehicle target speed. Goodness of results are meaning that the matching between simulated and target results, if these results valuable and if they can be considered to do an assessment for the methodology of looking-ahead strategy or not.

Table 5.1: Evaluation of the simulation results goodness

\begin{tabular}{|l|c|c|c|}
\hline Driving cycles & $\mathrm{R}^{2}$ & RMSE & Relative error (\%) \\
\hline Clmbs2Ind & 0.786 & 1.508 & -1.270 \\
\hline Las2Hall & 0.767 & 3.55 & -4.177 \\
\hline Hall2Bar & 0.910 & 1.552 & -1.61 \\
\hline BM2Sab & 0.993 & 1.189 & -2.444 \\
\hline Sab2BM & 0.956 & 2.415 & -6.738 \\
\hline
\end{tabular}

\subsection{Downsizing ESS energy opportunity with looking-ahead strategy}

Parallel hybrid electric vehicles use energy storage system as a secondary power source for the vehicle powertrain. The ESS size can impact significantly HEV effectiveness. For a conventional parallel HEV, the battery state of charge is sustained at the middle of active ESS capability. The reason for that is to give an equal chance for hybridization system to assist the vehicle powertrain whenever it is needed or to capture energy while using the brake system. However, with lookingahead strategy the vehicle propulsion controller can prepare the system in advance for forthcoming power demand if there was extra power in the engine to use. If the extra power that is needed for forthcoming traffic condition surpasses engine capability, then the VPC will charge the ESS as much as needed. Moreover, if the forthcoming traffic condition is a descending hill, then the VPC will guarantee that there is enough capacity in the ESS to capture that forthcoming free energy. In this section of the study, it was investigated the chance of downsizing ESS capacity (with looking-ahead strategy) while maintaining the same benefits in comparison to the original ESS capacity without looking-ahead control strategy. The first parallel HEV full load $($ GVWR $=36,000)$ model has equipment sizes of $150 \mathrm{~kW}$ and $10 \mathrm{kWh}$ for the electric motor and ESS respectively, and it is without the looking-ahead strategy. The second model is the same as the first model except the ESS is 5kWh, and it's with looking-ahead strategy. This proposed ESS downsizing is assuming that the engine power is still full size $(410 \mathrm{~kW})$. 
Figure (5.78) shows the impact of ESS energy downsizing on the activity of charging and discharging. Higher maximum and minimum for the state of charge are evident with the smaller ESS system. In other words, Downsizing the ESS energy results in increasing charging and discharging more frequently to meet the forthcoming power events. Charging and discharging the ESS more frequently was worse regarding efficiencies losses, as impacted fuel economy slightly. Although the parallel HEV with $10 \mathrm{kWh}$ battery size and without looking-ahead strategy was sufficient in assisting the engine and enough to capture free energy from using the brake pedal, but the smaller battery size $(5 \mathrm{kWh})$ was needed for looking-ahead strategy to manage the ESS in opportunistic regeneration as shown in Figure $(5.78 \mathrm{~b})$. Moreover, the total energy out from the ESS, with $10 \mathrm{kWh}$ and no ITS and with $5 \mathrm{kWh}$ and ITS, was different during the trip activities, but at the end of the trip the total energy out was close for both battery sizes as shown in Figure (5.78 c). Figure (5.79) shows the fuel economy for the two models on real world driving cycles. Fuel economy for the second model (with smaller ESS) was slightly different than the first model with $10 \mathrm{kWh}$ battery energy size as shown in Figure (5.80).

Figures (5.81and 5.82) show the amount of energy captured for the two models at different real world cycles and percentages of regenerative braking. These results show that there is a chance of downsizing ESS capacity (with looking-ahead strategy) with maintaining almost the same benefits. 

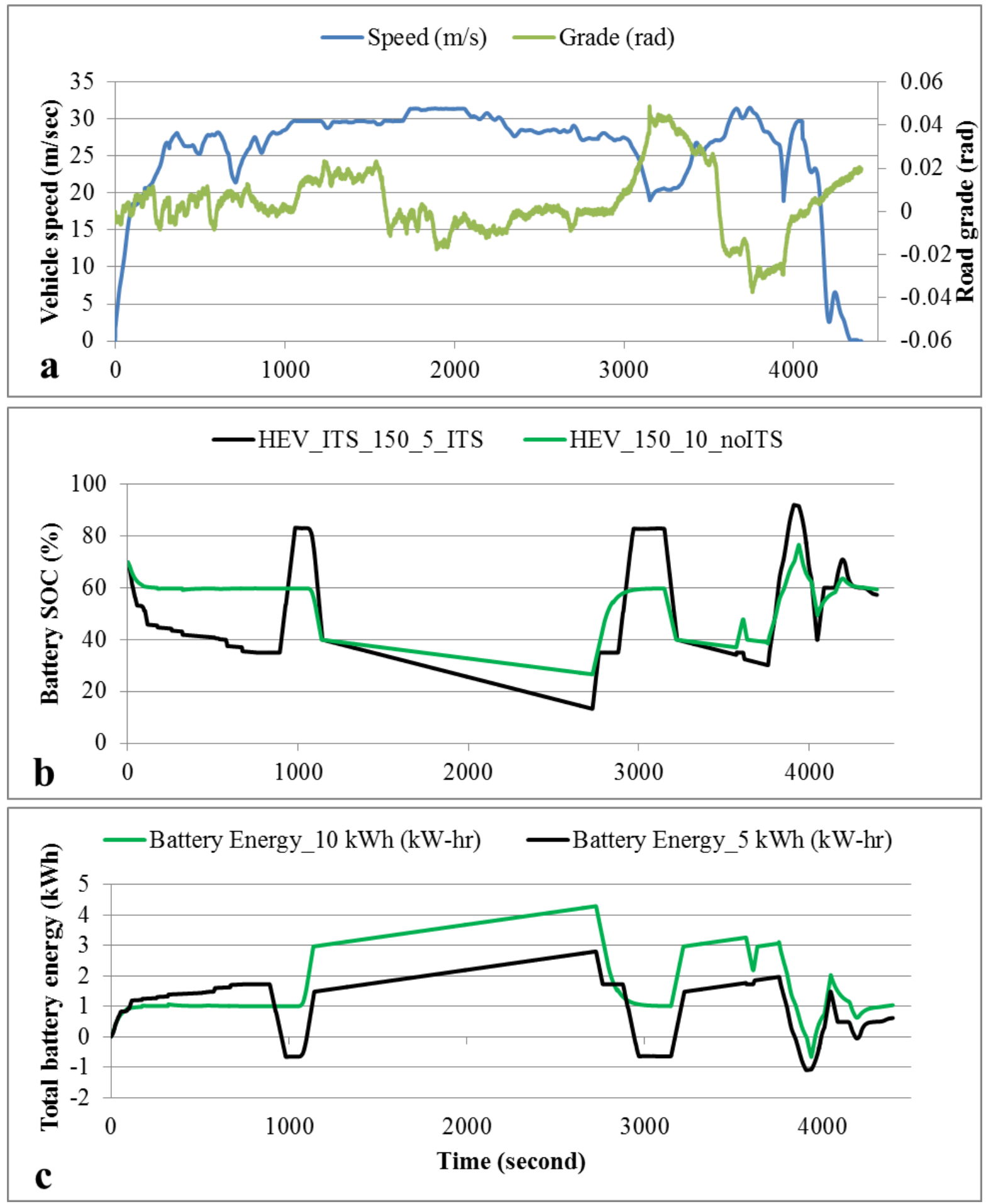

Figure 5.78: Battery SOC and total energy for parallel HEV model with full size battery and no ITS in comparison to parallel HEV model with smaller battery size and ITS at Las2Hall driving cycle 


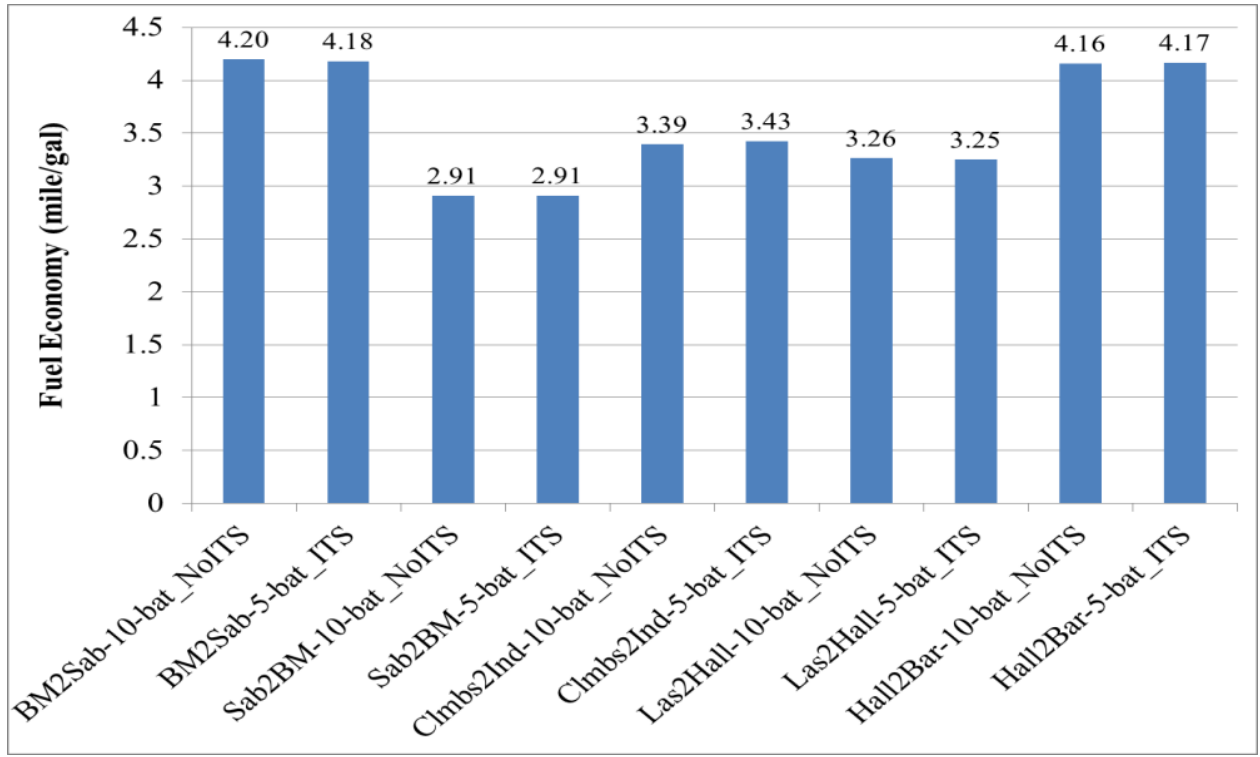

Figure 5.79: Fuel economy for parallel HEV model $(\mathrm{GVWR}=36,000 \mathrm{~kg})$ with $10 \mathrm{kWh}$ battery energy size (without looking-ahead strategy) and 5 kWh battery energy size (with looking-ahead strategy)

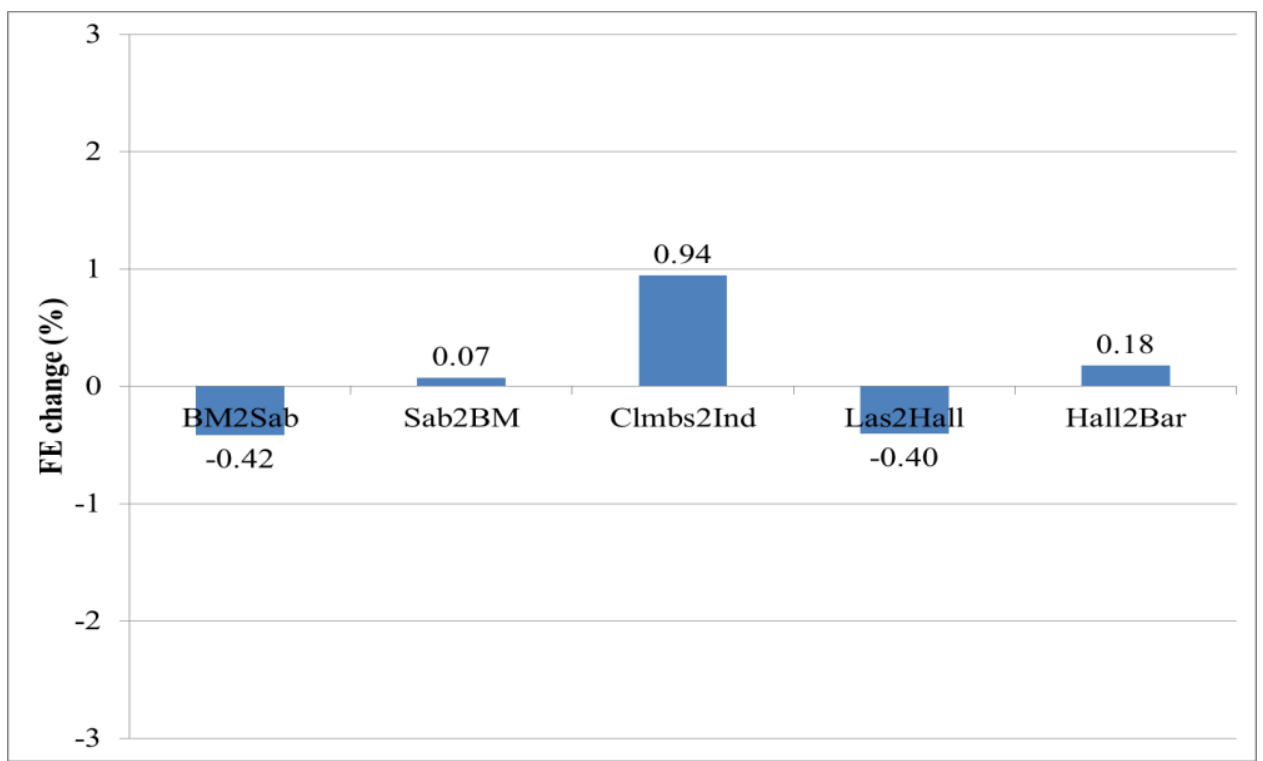

Figure 5.80: Fuel economy change for parallel HEV model (GVWR = $36,000 \mathrm{~kg}$ ) with $10 \mathrm{kWh}$ battery energy size (without looking-ahead strategy) and $5 \mathrm{kWh}$ battery energy size (with looking-ahead strategy) 


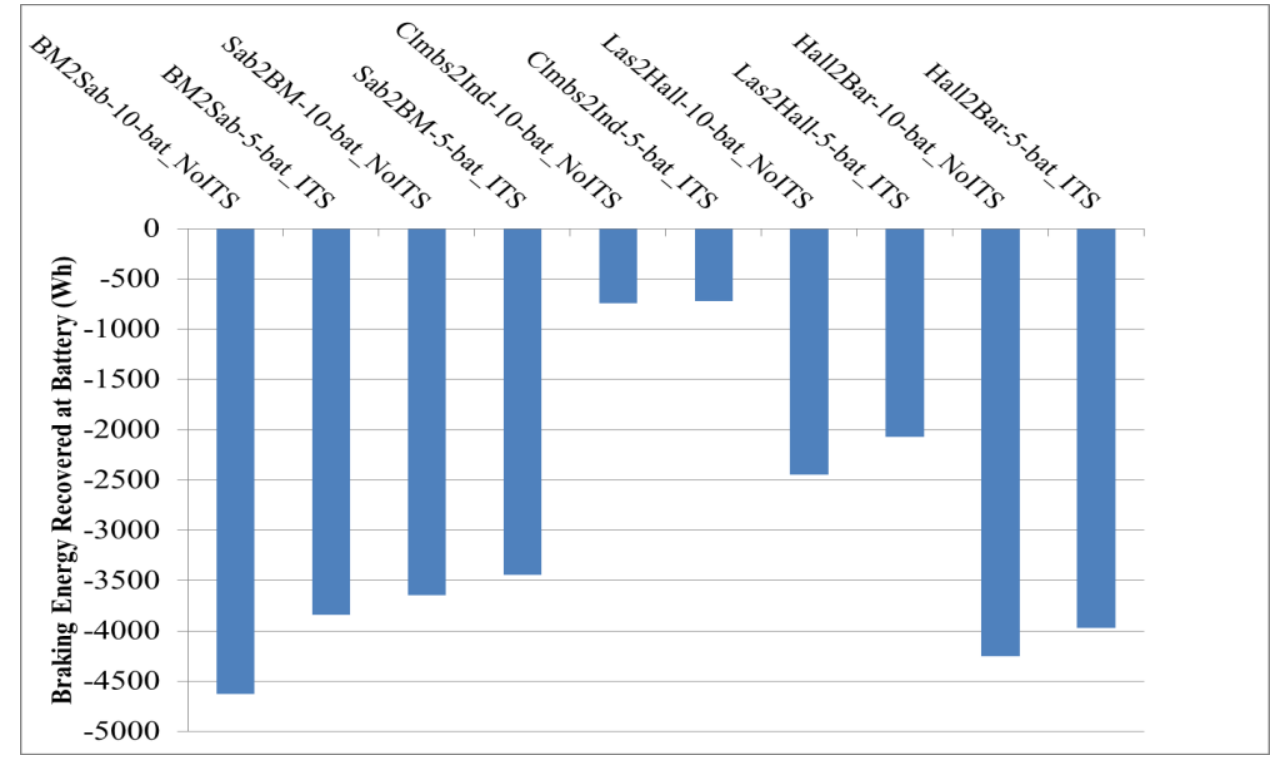

Figure 5.81: Braking energy recovered at battery for parallel HEV model $($ GVWR $=36,000 \mathrm{~kg}$ ) with $10 \mathrm{kWh}$ battery energy size (without lookingahead strategy) and $5 \mathrm{kWh}$ battery energy size (with looking-ahead strategy)

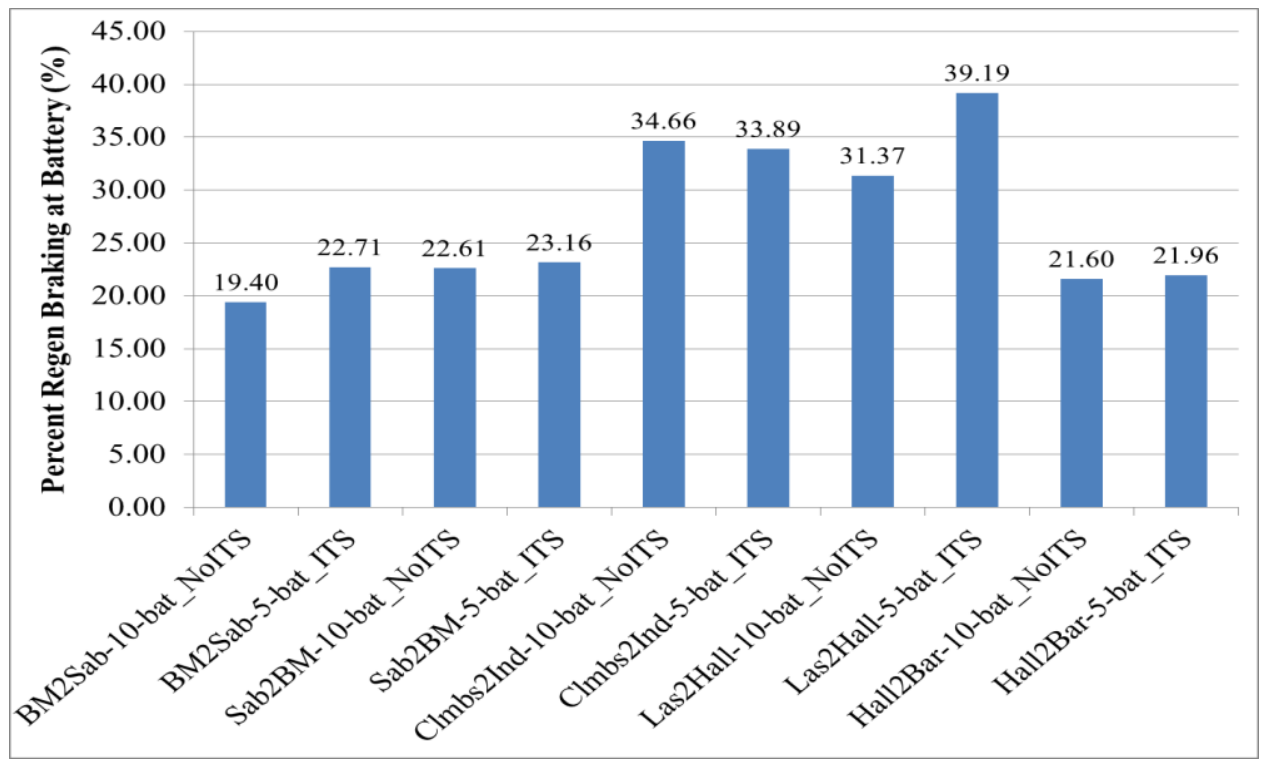

Figure 5.82: Percent regenerative braking battery for parallel HEV model $(\mathrm{GVWR}=36,000 \mathrm{~kg}$ ) with $10 \mathrm{kWh}$ battery energy size (without lookingahead strategy) and $5 \mathrm{kWh}$ battery energy size (with looking-ahead strategy) 


\subsection{Summary}

This section provides the results for expected outcomes from this study. The results show that there are different impacts of intelligent transportation systems information on parallel hybrid electric heavy duty vehicles. The impact of looking-ahead control strategy was noticeable on improving fuel economy, as much as $3 \%$, for specific terrain types. The impact of looking-ahead control strategy on improving fuel economy was a reverse proportional to the hybridization equipment sizes. Looking-ahead control strategy improves the chance to downsize engine power while maintaining almost the same total powertrain's power during different real world and chassis cycles. There was a limited vehicle speed compromised due to downsizing engine power, but the fuel economy improvement was about $4 \%$ (on real world flat terrain highway driving cycle) and up to $13 \%$ (on real world mountain terrain highway driving cycles). Looking-ahead strategy improved the chance to downsize battery energy to half with maintaining almost the same benefits (i.e. fuel economy and maintaining adequate power). Downsizing battery energy could decrease hybridization equipment costs. 


\section{Chapter 6 : Conclusions and Recommendations}

\subsection{Conclusions}

In this work, a parallel direct pre-transmission hybrid electric heavy duty vehicle configuration with and without looking-ahead control strategy was investigated for a range of vehicle masses, battery sizes and motor/generator sizes. The results revealed that looking-ahead control strategy for heavy duty parallel HEV can contribute preparing the alternative power source in advance. The other specific findings of the study can be summarized as follows:

The results of running the models on constant speed highway driving cycles with different road grades have revealed the impact of the proposed algorithm on ESS management in opportunistic regeneration based on the forthcoming total power demand estimation (negative or positive).

> The looking-ahead strategy has shown an impact on managing the alternative power source for the HEV. In other words, the looking-ahead VPC switches between sustaining and depleting battery SOC strategies based on the forthcoming traffic conditions.

For the parallel hybrid electric heavy duty vehicle with $50 \%$ load, the fuel economy change with looking-ahead control strategy has been improved from $0.5 \%$ on flat terrain up until $3 \%$ for descending hills terrain in comparison to the same parallel HEV model without lookingahead strategy.

Looking-ahead strategy can give a chance reducing the hybridization equipment sizes with improving fuel economy and maintain same benefits in comparison to the original equipment sizes without looking-ahead.

Looking-ahead strategy has shown potential for downsizing engine power (410 to $310 \mathrm{~kW}$ ) while maintaining almost the same adequate power (finished the same route at the same time). Fuel economy is improved up to $13 \%$ on mountain terrain in comparison to the same parallel HEV model with full size engine power $(410 \mathrm{~kW})$.

Intelligent transportation systems information could give a chance to decrease parallel HEV cost by downsizing battery energy to half, with keeping all other equipment sizes relative to parallel HEV without ITS, while maintaining almost the same benefits (i.e. fuel economy and maintaining adequate power). 


\subsection{Recommendations}

Very likely the results for this study seem promising and show that the benefits from lookingahead strategy are expected. All these evaluations are based on simulation of parallel HEV model (Autonomie software) with different equipment sizes. The most important recommendation is to test a parallel hybrid electric vehicle with looking-ahead control strategy on chassis dynamometer or real world terrains. On the other hand, to get these real world conditions tests done, the real time communication from infrastructure to vehicle-on road (I2V) should be available, which it is not available now (2013). This forthcoming traffic information could simply be programmed into the dynamometer instructions as input to resemble the signal information from a real $\mathrm{I} 2 \mathrm{~V}$ system. Moreover, this research shows that there is possibility of more improvement of the VPC strategy to enable it dealing with more traffic information details in comparison to currently information available, which suggested and used in this study. There are chances to improve looking-ahead control strategy for hybrid electric truck by manipulating the method of VPC of managing the alternative power source. For example, the new models of sport utility light duty vehicle (SUV) have a button to switch between sport and economic driving modes. In other words, truck drivers might be able to decide if they need to increase fuel economy on account of maintaining adequate total power during all activities. 


\section{References}

1. Harrington, W. and Krupnick, A. "Improving fuel economy in heavy-duty vehicles." (March 28, 2012). Resources for the Future DP 12-02. Available at SSRN: http://ssrn .com/abstract=2038842 or http://dx.doi.org/10.2139/ssrn. 2038842

2. Cawthorne, W. R., Famouri, P., Chen, J., Clark, N. N., McDaniel, T. I., Atkinson, R. J., Nandkumar,S., Atkinson, C. M., and Petreanu, S. "Development of a linear alternatorengine for hybrid electric vehicle applications." IEEE Transactions on Vehicular Technology, 48(6). pp.1797-1802, 1999.

3. Gruenwald, R., Major, J. T., and Palumbo, A. J. "Hybrid electric vehicle." U.S. Patent. 6,651,759; 25 Nov. 2003

4. Gruenwald, R., Major, J. T., and Palumbo, A. J. "Hybrid electric vehicle." U.S. Patent. 7,004,273; 28 Feb. 2006

5. Gruenwald, R., Major, J. T., and Palumbo, A. J. "Hybrid electric vehicle." U.S. Patent. 7,252,165; 7 Aug. 2007

6. Lin, C. C., Peng, H., Grizzle, J. W., and Kang, J. M. "Power management strategy for a parallel hybrid electric truck." in IEEE Transactions on Control Systems Technology, 11(6). pp. 839-849, 2003.

7. Severinsky, A. J. "Hybrid electric vehicle." U.S. Patent. 5,343,970; 6 Sep.1994.

8. Kawamura, H. "Hybrid electric vehicle." U.S. Patent. 5,881,559; 16 Mar. 1999.

9. Sciarretta, A., Back, M., and Guzzella, L. "Optimal control of parallel hybrid electric vehicles." IEEE Transactions on Control Systems Technology, 12(3). pp. 352-363, 2004.

10. Paganelli, G., Guerra, T. M., Delprat, S., Santin, J. J., Delhom, M., and Combes, E. "Simulation and assessment of power control strategies for a parallel hybrid car." Proceedings of the Institution of Mechanical Engineers, Part D: Journal of Automobile Engineering 214(7). pp. 705-717, 2000.

11. Assanis, D. N., Bryzik, W., Chalhoub, N., Filipi, Z., Henein, N., Jung, D., Liu, X., Louca, L.,Moskwa, J., Munns, S., Overholt, J., Papalambros, P., Riley, S., Rubin, Z., Sendur, P., Stein, J., and Zhang, G."Integration and use of diesel engine, driveline and vehicle dynamics models for heavy duty truck simulation." No. 1999-01-0970. SAE Technical Paper, 1999.

12. Block, M., Clark, N., Wayne, S., Nine, R., and Miller, W. "An Investigation into the Emissions Reduction Performance of an SCR System Over Two Years' In-Use HeavyDuty Vehicle Operation." No. 2005-01-1861. SAE Technical Paper, 2005. 
13. Wang, J., Cai, L., Wang, Y., Ma, Y., and Zhang, X. "Modeling and optimization matching on drive system of a coaxial parallel-type hybrid-power gas engine heat pump." Energy 55 (2013): pp. 1196-1204.

14. Hellström, E., Ivarsson, M., Åslund, J., and Nielsen, L. "Look-ahead control for heavy trucks to minimize trip time and fuel consumption." Control Engineering Practice 17(2). pp. 245-254, 2009.

15. Kleimaier, A., and Schroder, D. "Optimization strategy for design and control of a hybrid vehicle." in 6th International Workshop on Advanced Motion Control, 2000. Proceedings. (pp. 459-464). IEEE, 2000.

16. Kirschbaum, F., Back, M., and Hart, M. "Determination of the fuel-optimal trajectory for a vehicle along a known route." In $15^{\text {th }}$ IFAC World Congress (Vol. 15, No. 1, pp. 15051505), 2002.

17. Washington, G., Rizzoni, G., and Guezennec, Y. "Development of fuzzy logic and neural network control and advanced emissions modeling for parallel hybrid vehicles": National Renewable Energy Laboratory. 2003.

18. West, M. J., Bingham, C. M., and Schofield, N. "Predictive control for energy management in all/more electric vehicles with multiple energy storage units." In Electric Machines and Drives Conference, 2003. IEMDC'03. IEEE International (Vol. 1, pp. 222228). IEEE.

19. "Advanced Traffic Management Systems (ATMS)." [cited April 12 2013]; Available from: http://www.swri.org/4org/d10/isd/atms/. .

20. Rajagopalan, A. and Washington, G. "Intelligent control of hybrid electric vehicles using GPS information." No. 2002-01-1936. SAE Technical Paper, 2002.

21. Kisacikoglu, M. C., Uzunoglu, M., and Alam, M. S. "Load sharing using fuzzy logic control in a fuel cell/ultracapacitor hybrid vehicle." International Journal of Hydrogen Energy 34(3). pp. 1497-1507, 2009.

22. Deguchi, Y., Kuroda, K., Shouji, M., and Kawabe, T. "HEV charge/discharge control system based on navigation information." No. 2004-21-0028. SAE Technical Paper, 2004.

23. "Hybrid electric transportation propulsion \& power system". BAE system [cited July 21 2013]; Available from: http://www.hybridrive.com/default.asp

24. Simpson, A. G. "Parametric modelling of energy consumption in road vehicles": $\mathrm{PhD}$ Thesis, University of Queensland. 2005.

25. Barth, M., Younglove, T., and Scora, G."Development of a heavy-duty diesel modal emissions and fuel consumption model." California Partners for Advanced Transit and Highways (PATH) (2005). 
26. Giannelli, R. A., Nam, E. K., Helmer, K., Younglove, T., Scora, G., and Barth, M. "Heavy-duty diesel vehicle fuel consumption modeling based on road load and power train parameters." No. 2005-01-3549. SAE Technical Paper, 2005.

27. Hucho, W. H. and Sovran, G. "Aerodynamics of road vehicles." Annual review of fluid mechanics 25.1 (1993): 485-537.

28. Wayne, W. S., Clark, N. N., Nine, R. D., and Elefante, D. "A comparison of emissions and fuel economy from hybrid-electric and conventional-drive transit buses." Energy \& Fuels 18(1). pp. 257-270, 2004.

29. Kelly, K. J. "Modeling tools for predicting the impact of rolling resistance on energy usage and fuel efficiency for realistic driving cycles." Transportation 13: 30, 2000.

30. Petrushov, V. A. "Improvement in vehicle aerodynamic drag and rolling resistance determination from coast-down tests." Proceedings of the Institution of Mechanical Engineers, Part D: Journal of Automobile Engineering 212(5). pp. 369-380, 1998.

31. Park, J. H., Kong, J. H., Jo, H. S., Park, Y. I., and Lee, J. M. "Measurement of road gradients for the development of driving modes including road gradients." Proceedings of the Institution of Mechanical Engineers, Part D: Journal of Automobile Engineering 215(9). pp. 977-986, 2001.

32. Fitzpatrick, K., and Wooldridge, M. "Recent geometric design research for improved safety and operations." No. Project 20-5 FY 1999, 2001.

33. Walston, W. H., Buckley, F. T., and Marks, C. H. "Test procedures for the evaluation of aerodynamic drag on full-scale vehicles in windy environments" No. 760106. SAE Technical Paper, 1976.

34. Burke, A. F. "Hybrid/electric vehicle design options and evaluations." No. 920447. SAE Technical Paper, 1992.

35. Karbowski, D., Delorme, A., and Rousseau, A. "Modeling the hybridization of a class 8 line-haul truck." No. 2010-01-1931. SAE Technical Paper, 2010.

36. Ferreira, A. A., Pomilio, J. A., Spiazzi, G., and de Araujo Silva, L. "Energy management fuzzy logic supervisory for electric vehicle power supplies system." IEEE Transactions on Power Electronics, 23(1). pp. 107-115, 2008.

37. Jalil, N., Kheir, N. A., and Salman, M. "A rule-based energy management strategy for a series hybrid vehicle." In Proceedings of the 1997 American Control Conference. (Vol. 1, pp. 689-693). IEEE. 1997.

38. Schoeggl, P., Ramschak, E., and Bogner, E. "On-board optimization of driveability character depending on driver style by using a new closed loop approach." No. 2001-010556. SAE Technical Paper, 2001. 
39. Tehranian, A. "Effects of artificial neural networks characterization on prediction of diesel engine emissions." PhD diss., West Virginia University, 2003.

40. Sciarretta, A., Back, M., and Guzzella, L. "Optimal control of parallel hybrid electric vehicles." IEEE Transactions on Control Systems Technology, 12(3). pp. 352-363, 2004.

41. Turlapati, V. V. K. "Modeling and optimization of a plug-in hybrid electric vehicle", MSc. thessis, Clemson University, 2010.

42. Markel, T., Brooker, A., Hendricks, T., Johnson, V., Kelly, K., Kramer, B., O’Keefe, M., Sprik, S., and Wipke, K. "ADVISOR: a systems analysis tool for advanced vehicle modeling." Journal of Power Sources 110(2). pp. 255-266, 2002.

43. Cole, G. H. "SIMPLEV: A simple electric vehicle simulation program, Version 1.0", No. DOE-ID-10293. 1991.

44. Ciccarelli, T., Toossi, R., and Goodarzi, A. "Assessment of hybrid configuration and control strategies in planning future metropolitan/urban transit systems" No. 2001-012502. SAE Technical Paper, 2001.

45. "Autonomie training part 1 overview". Argonne National Laboratory [cited April/ 21/ 2013]; Available from: http://www.autonomie.net/docs/7\%20-\%20Training/ training part1.pdf.

46. Alamgir, M., and Sastry, A. M. "Efficient batteries for transportation applications." No. 2008-21-0017. SAE Technical Paper, 2008.

47. Plett, G. L. "Extended kalman filtering for battery management systems of LiPB-based HEV battery packs: Part 1. background." Journal of Power Sources 134(2). pp. 252-261, 2004.

48. Smith, K. and Wang, C. Y. "Power and thermal characterization of a lithium-ion battery pack for hybrid-electric vehicles." Journal of Power Sources 160(1). pp. 662-673, 2006.

49. Aditya, J. P. and Ferdowsi, M. "Comparison of NiMH and Li-ion batteries in automotive applications." in Vehicle Power and Propulsion Conference, 2008. VPPC'08. IEEE (pp. 1-6). IEEE. 2008.

50. Lu, L., Han, X., Li, J., Hua, J., and Ouyang, M. "A review on the key issues for lithiumion battery management in electric vehicles." Journal of Power Sources 226: pp. 272-288, 2013.

51. "Capacitor Characteristics". Rod Elliott (ESP) 2005 [cited July/20/2013]; Available from: http://sound.westhost.com/articles/capacitors.htm\#2.0.

52. Rotenberg, D., Vahidi, A., and Kolmanovsky, I. "Ultracapacitor assisted powertrains: modeling, control, sizing, and the impact on fuel economy." IEEE Transactions on Control Systems Technology, 19(3). pp. 576-589, 2011. 
53. "Autonomie overview ", Argonne National Laboratory. [cited March/21/2013]; Available from: http://www. Autonomie.net/overview/index.html.

54. "Autonmie software", in Argonne TTRDC2012, Argonne National Laboratory: USA. authorized to Ahmed Al-Samari, 2012-2013.

55. Wang, L., Clark, N., and Chen, P. "Modeling and validation of an over-the-road truck." No. 2010-01-2001. SAE Technical Paper, 2010.

56. Delgado, O. F., Clark, N. N., and Thompson, G. J. "heavy duty truck fuel consumption prediction based on driving cycle properties." International Journal of Sustainable Transportation 6(6). pp. 338-361, 2012.

57. Delgado-Neira, O. F. "Driving cycle properties and their influence on fuel consumption and emissions": PhD diss., West Virginia University, 2012.

58. "A quick derivation relating altitude to air pressure". Portland State Aerospace Society (PSAS) 2004 [cited Jan/15/2013]; Available from: http://psas.pdx.edu/RocketScience/ PressureAltitude_Derived.pdf.

59. Aashto, A. "Policy on geometric design of highways and streets." American Association of State Highway and Transportation Officials, Washington, DC, 1, 990, 2001.

60. Zeraoulia, M., Benbouzid, M. E. H., and Diallo, D. "Electric motor drive selection issues for HEV propulsion systems: A comparative study." IEEE Transactions on Vehicular Technology, 55(6). pp. 1756-1764, 2006.

61. Al-Alawi, B. M., and Bradley, T. H. "Review of hybrid, plug-in hybrid, and electric vehicle market modeling Studies." Renewable and Sustainable Energy Reviews 21: pp. 190-203, 2013.

62. Zhu, W. H., Zhu, Y., Davis, Z., and Tatarchuk, B. J. "Energy efficiency and capacity retention of Ni-MH batteries for storage applications." Applied Energy 106: pp. 307313, 2013.

63. Rajashekara, K. "Present status and future trends in electric vehicle propulsion technologies." IEEE Journal of Emerging and Selected Topics in Power Electronics 1(1). pp. 3-10, 2013.

64. "Inovation solutions for electrifying vehicles". UQM Technologies [cited June 8 2013]; Available from: http://uqm.com/technology/.

65. Berton, J. J., Freeh, J. E., and Wickenheiser, T. J. "An analytical performance assessment of a fuel cell-powered, small electric airplane." in Symposium on Novel and Emerging Vehicle Technology Concepts, Brussels, Belgium. 2003. 
66. Ed Bargar, H., Li, J., Goering, D. J., and Lee, J. H. "Modeling and verification of hybrid electric HMMWV performance." in Industrial Electronics Society, 2003. IECON'03. The 29th Annual Conference of the IEEE (Vol. 1, pp. 939-944). IEEE. 2003.

67. Williamson, S. S., Lukic, S. M., and Emadi, A. "Comprehensive drive train efficiency analysis of hybrid electric and fuel cell vehicles based on motor-controller efficiency modeling." IEEE Transactions on Power Electronics, 21(3). pp. 730-740, 2006.

68. Lutsey, N., and Sperling, D. "Energy efficiency, fuel economy, and policy implications." transportation research record: Journal of the Transportation Research Board 1941(1). pp. 8-17, 2005.

69. Wang, L. "Heavy-duty vehicles models and factors impacting fuel consumption", $\mathrm{PhD}$ diss., West Virginia University, 2011.

70. Rakha, H., Snare, M., and Dion, F. "Vehicle dynamics model for estimating maximum light-duty vehicle acceleration levels." transportation research record: journal of the transportation research board 1883(1). pp. 40-49, 2004.

71. Chen, X., Schmid, N. A., Wang, L., and Clark, N. N. "Regression-based oxides of nitrogen predictors for three diesel engine technologies." Journal of the Air \& Waste Management Association 60(1). pp. 72-90, 2010.

72. Piller, S., Perrin, M., and Jossen, A. "Methods for state-of-charge determination and their applications." Journal of Power Sources 96(1). pp. 113-120, 2001. 


\section{Appendix A: Simulation results goodness}

\section{A.1: Conventional vehicle at full load}

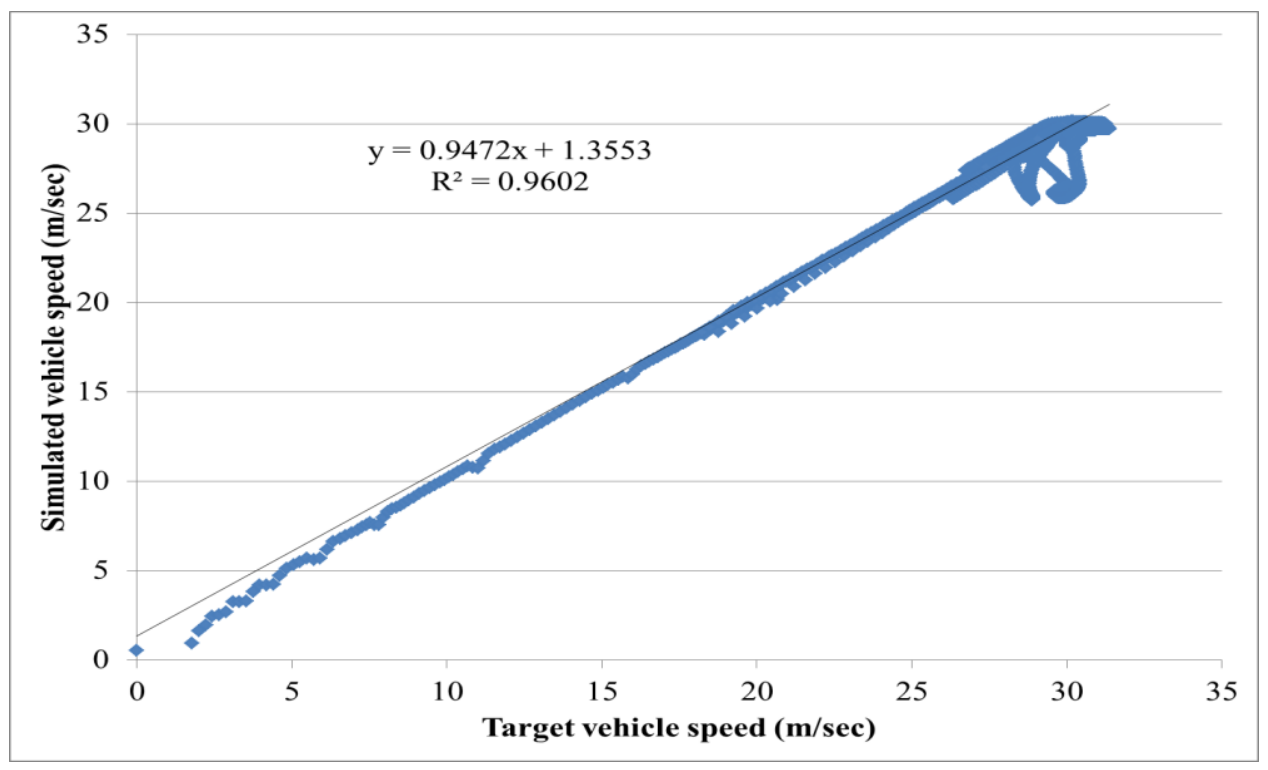

Figure A.1: Speed simulation results goodness for a conventional model at Clmb2Ind real world driving cycle

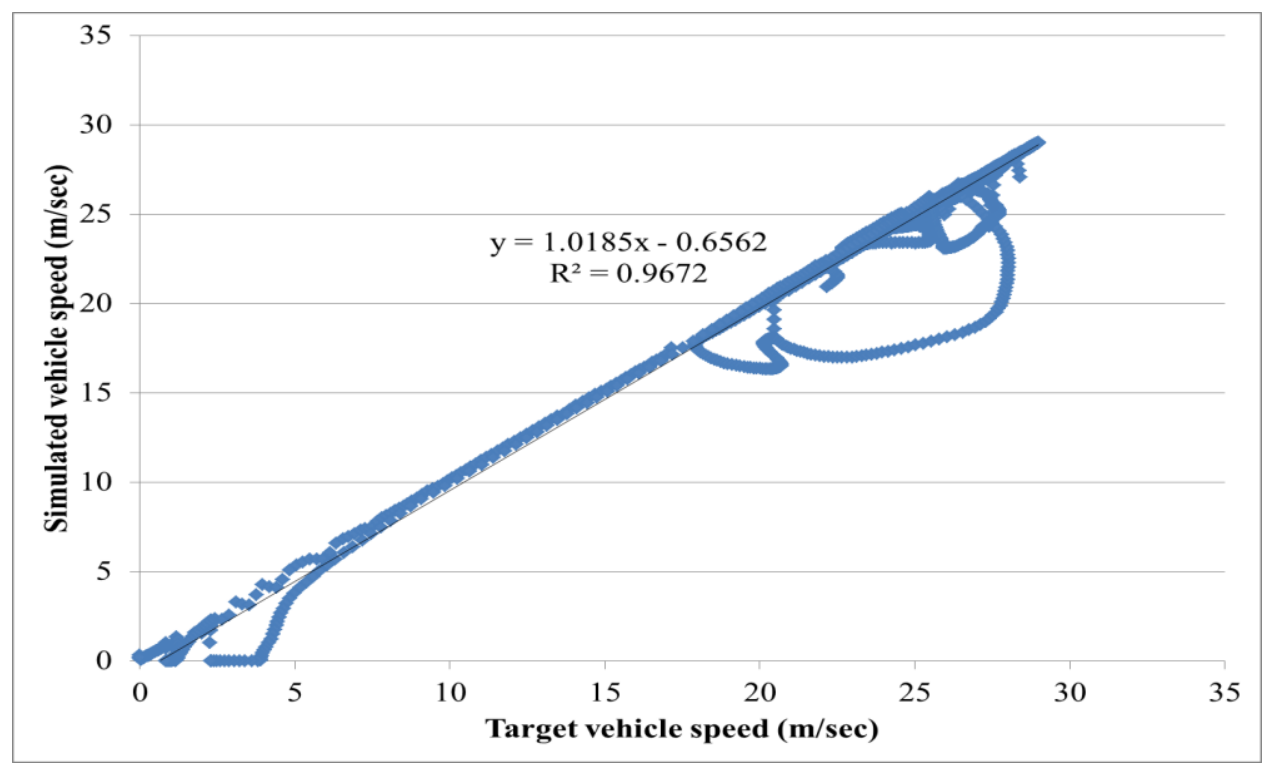

Figure A.2: Speed simulation results goodness for a conventional model at Hall2Bar real world driving cycle 


\section{A.2: HEV_150_10_with NoITS at full load}

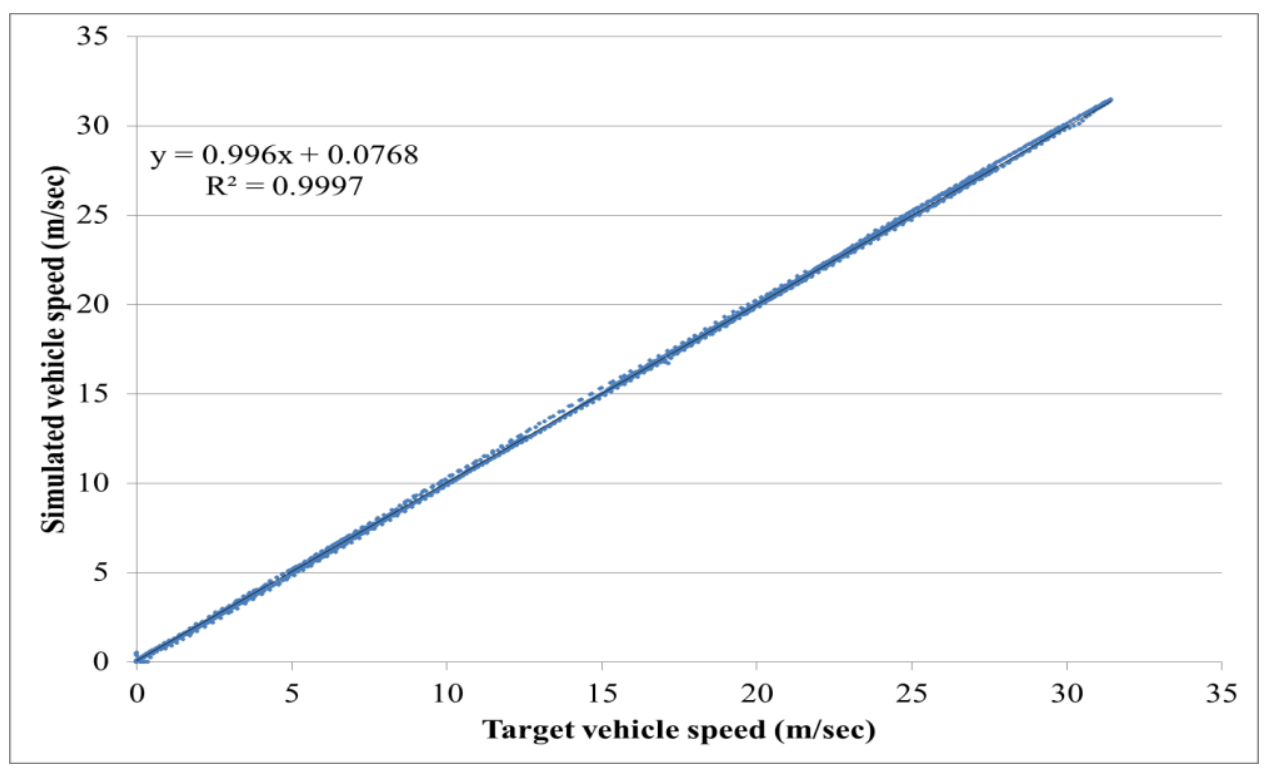

Figure A.3: Speed simulation results goodness for a HEV with NoITS model at BM2Sab real world driving cycle

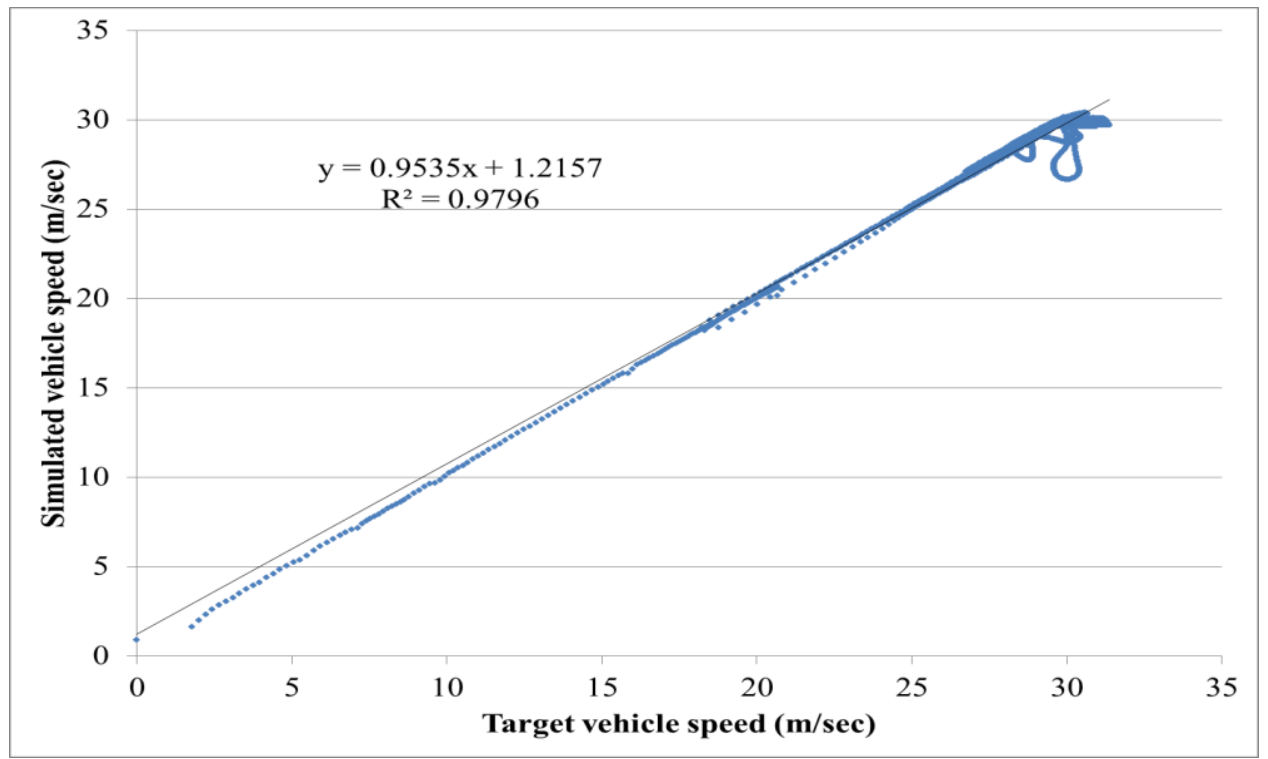

Figure A.4: Speed simulation results goodness for a HEV with NoITS model at Clmb2IND real world driving cycle 


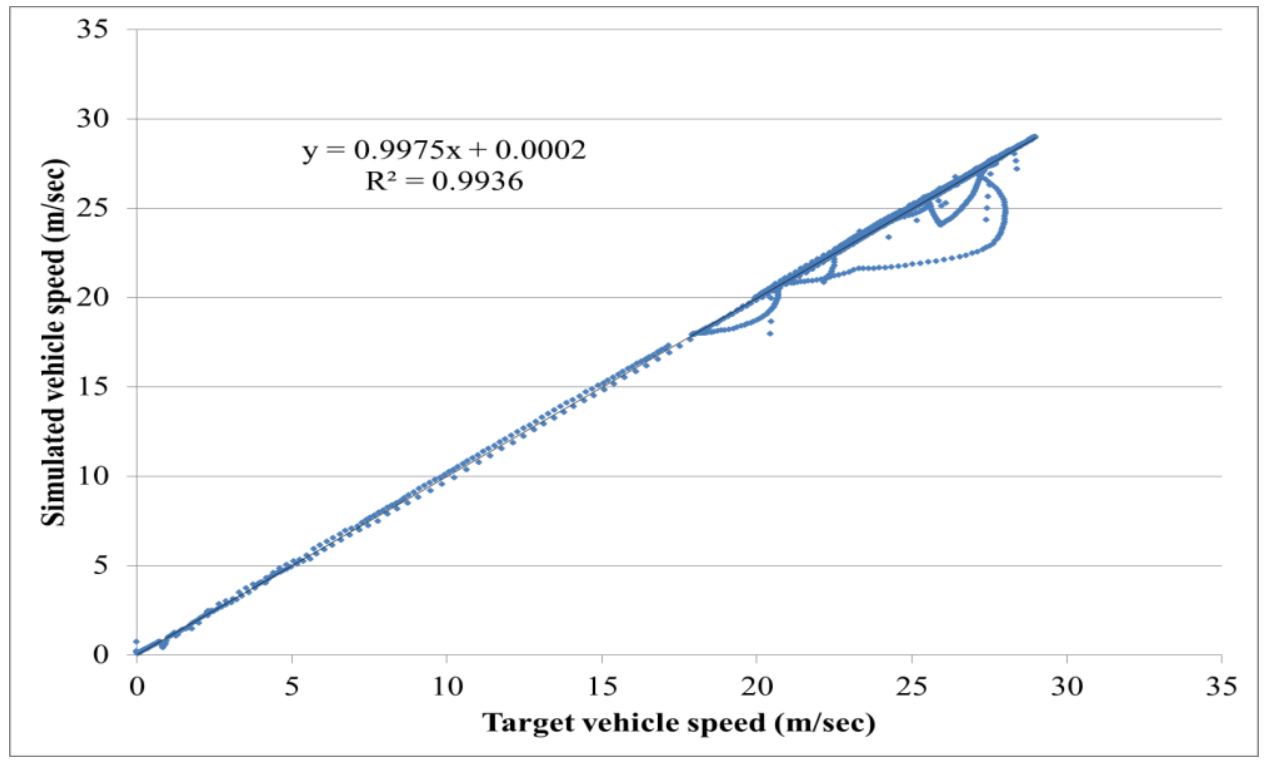

Figure A.5: Speed simulation results goodness for a HEV with NoITS model at Hall2Bar real world driving cycle

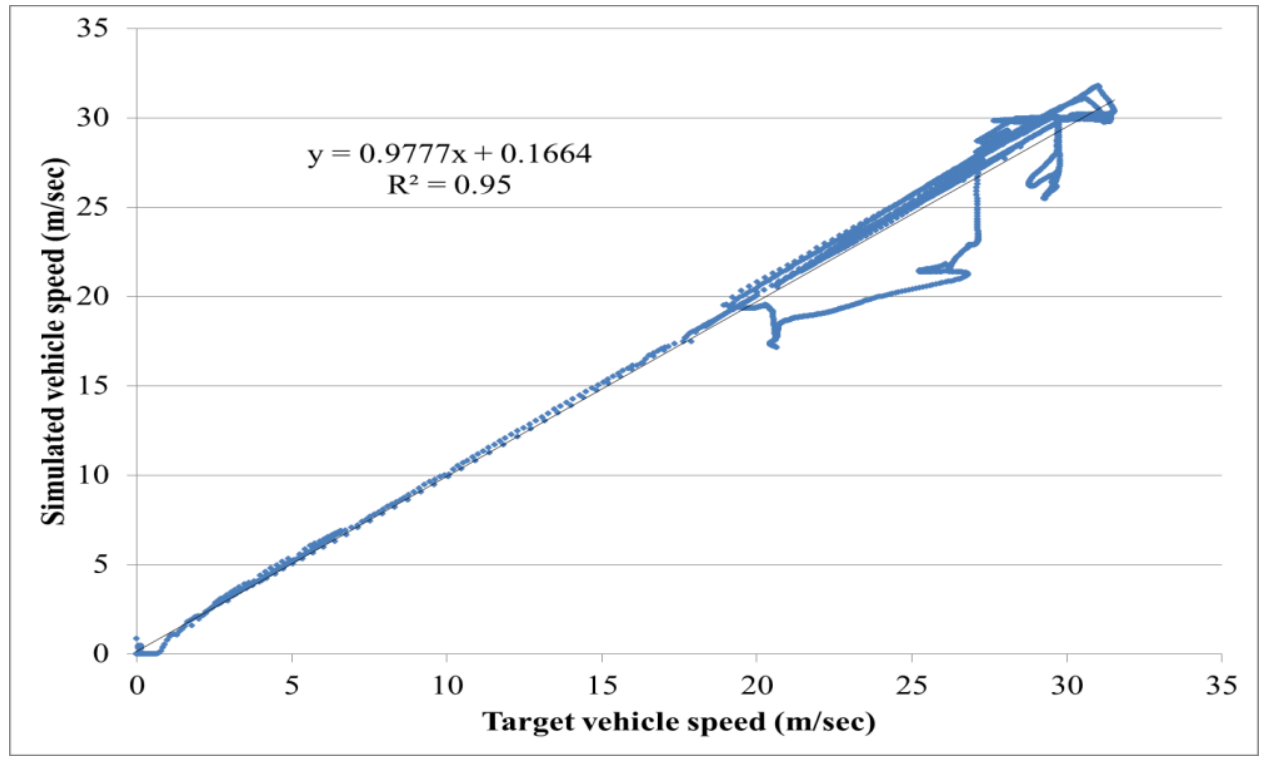

Figure A.6: Speed simulation results goodness for a HEV with NoITS model at Las2Hall real world driving cycle 


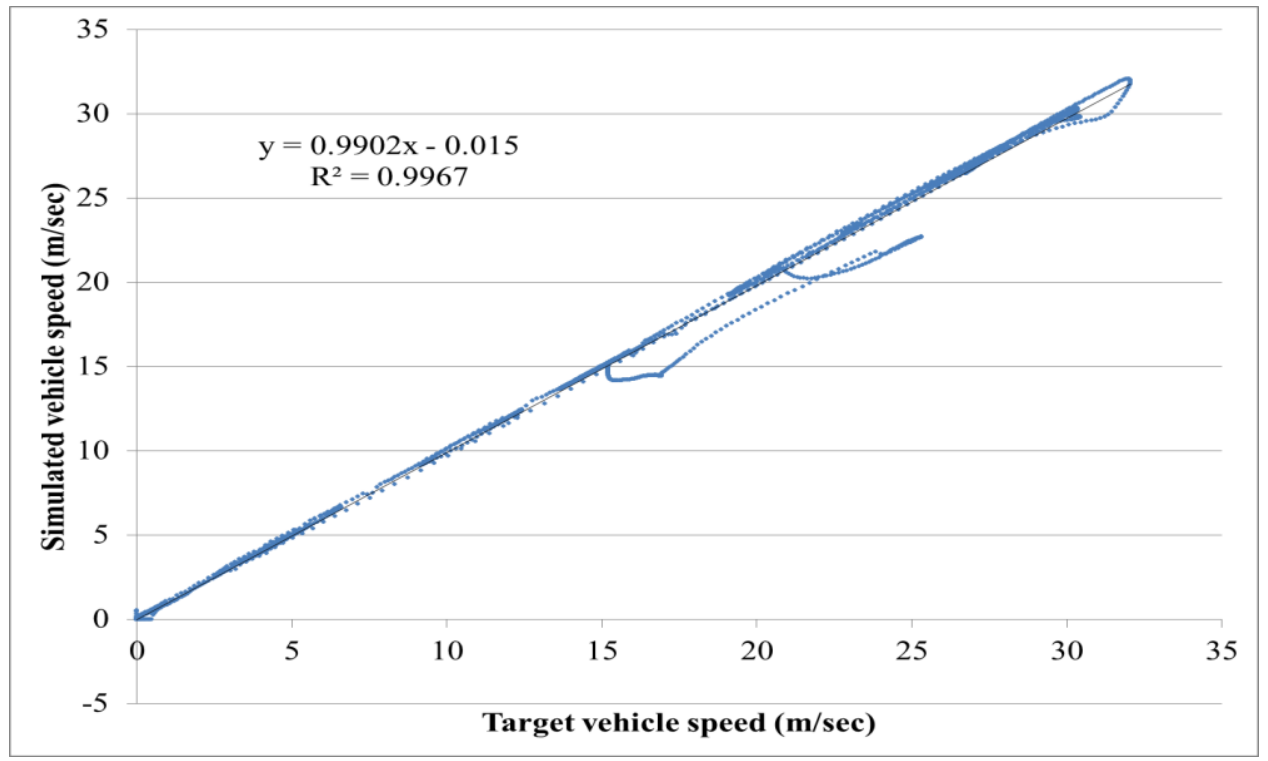

Figure A.7: Speed simulation results goodness for a HEV with NoITS model at Sab2BM real world driving cycle

\section{A.3: HEV_150_10_with ITS at full load}

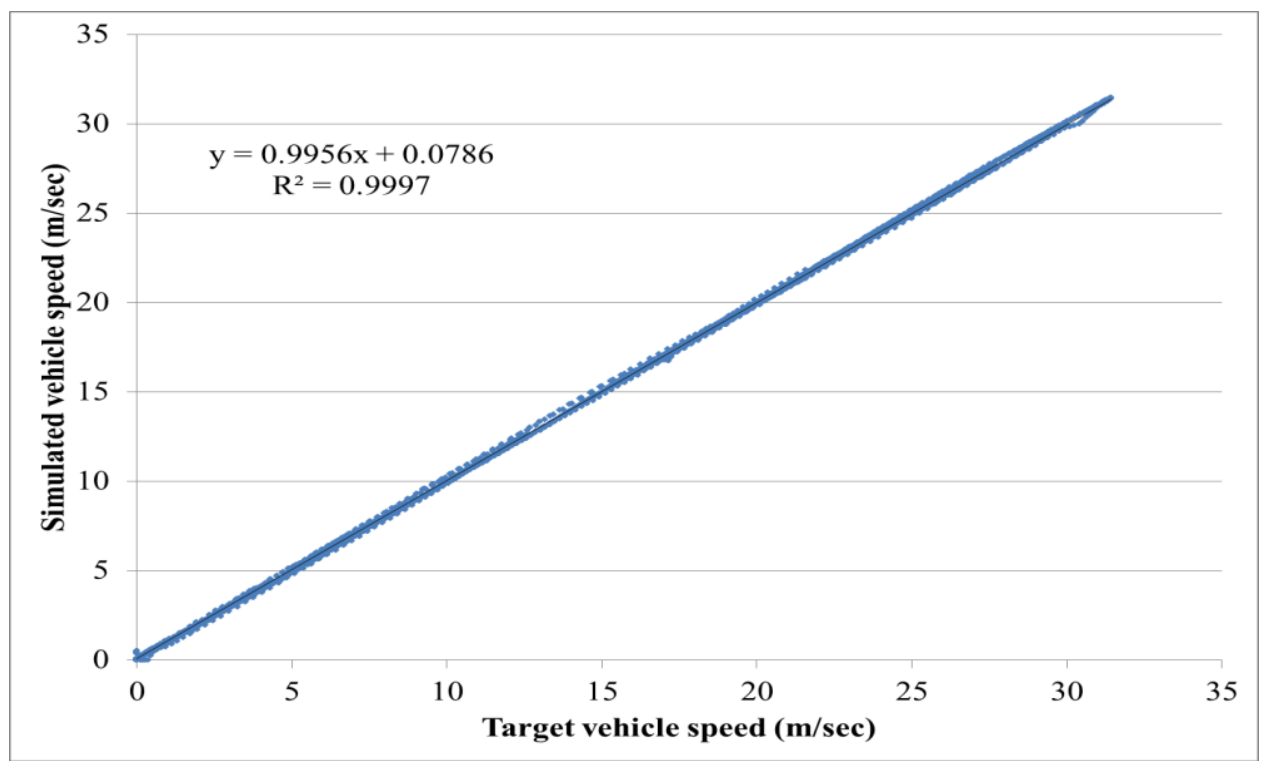

Figure A.8: Speed simulation results goodness for a HEV with ITS model at BM2Sab real world driving cycle 


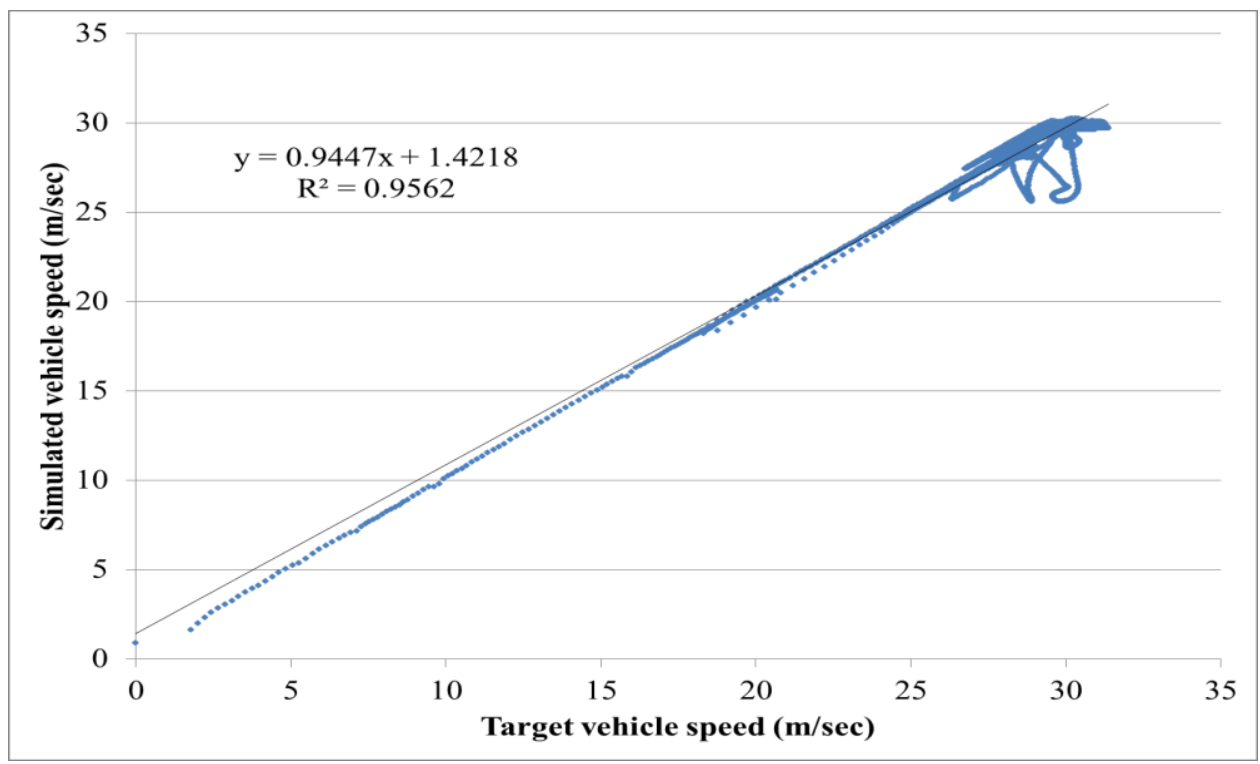

Figure A.9: Speed simulation results goodness for a HEV with ITS model at Clmb2Ind real world driving cycle

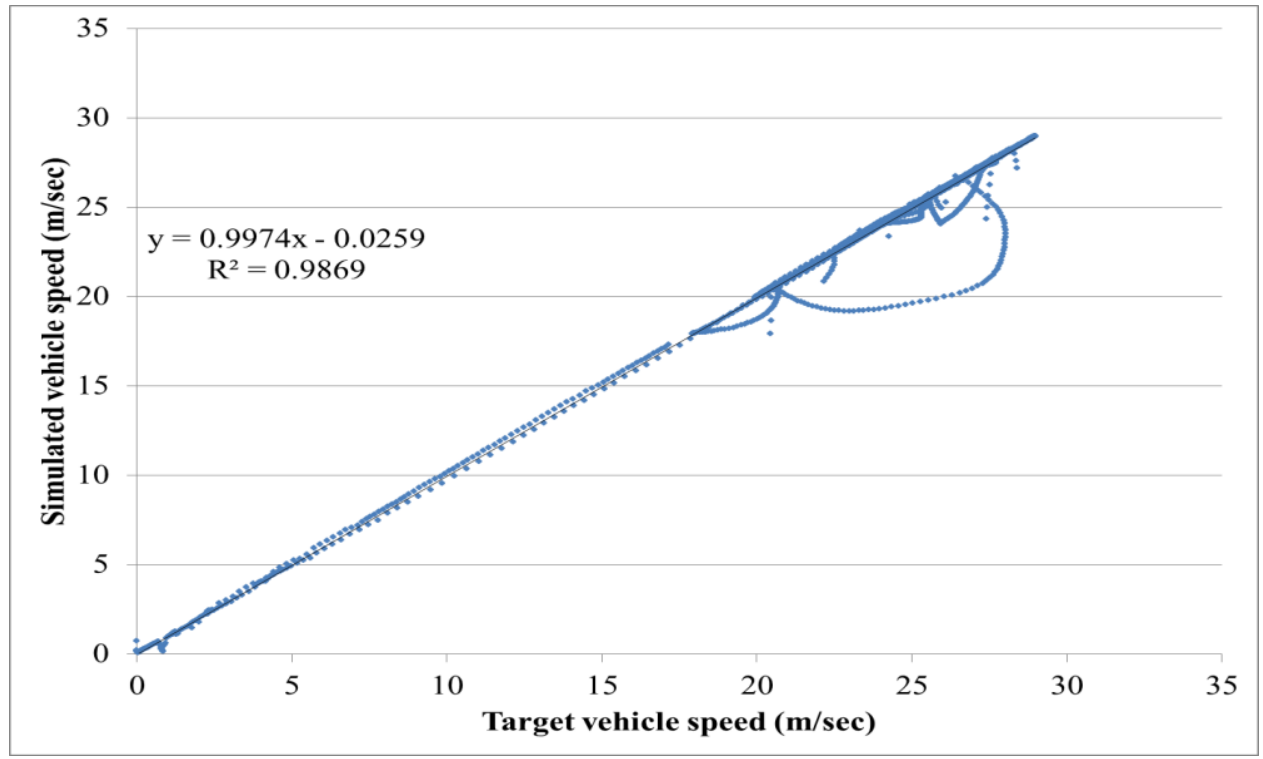

Figure A.10: Speed simulation results goodness for a HEV with ITS model at Hall2Bar real world driving cycle 


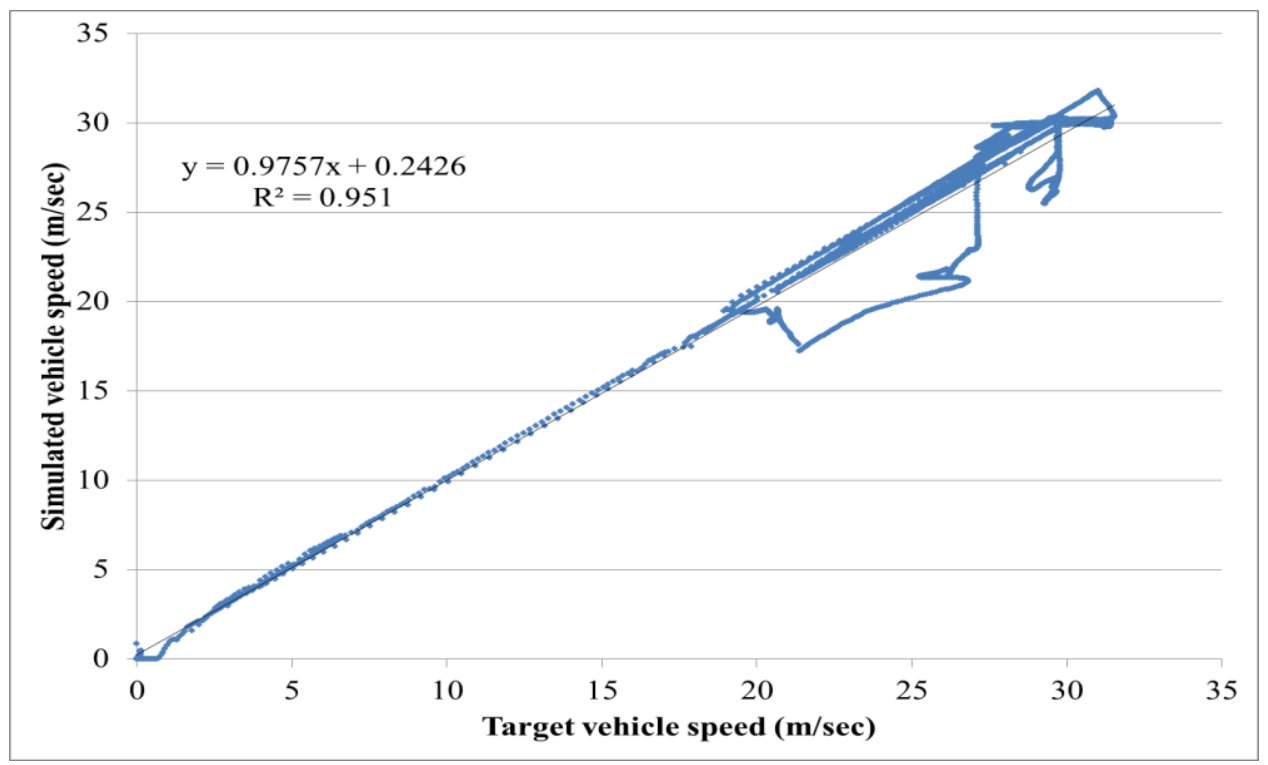

Figure A.11: Speed simulation results goodness for a HEV with ITS model at Las2Bar real world driving cycle

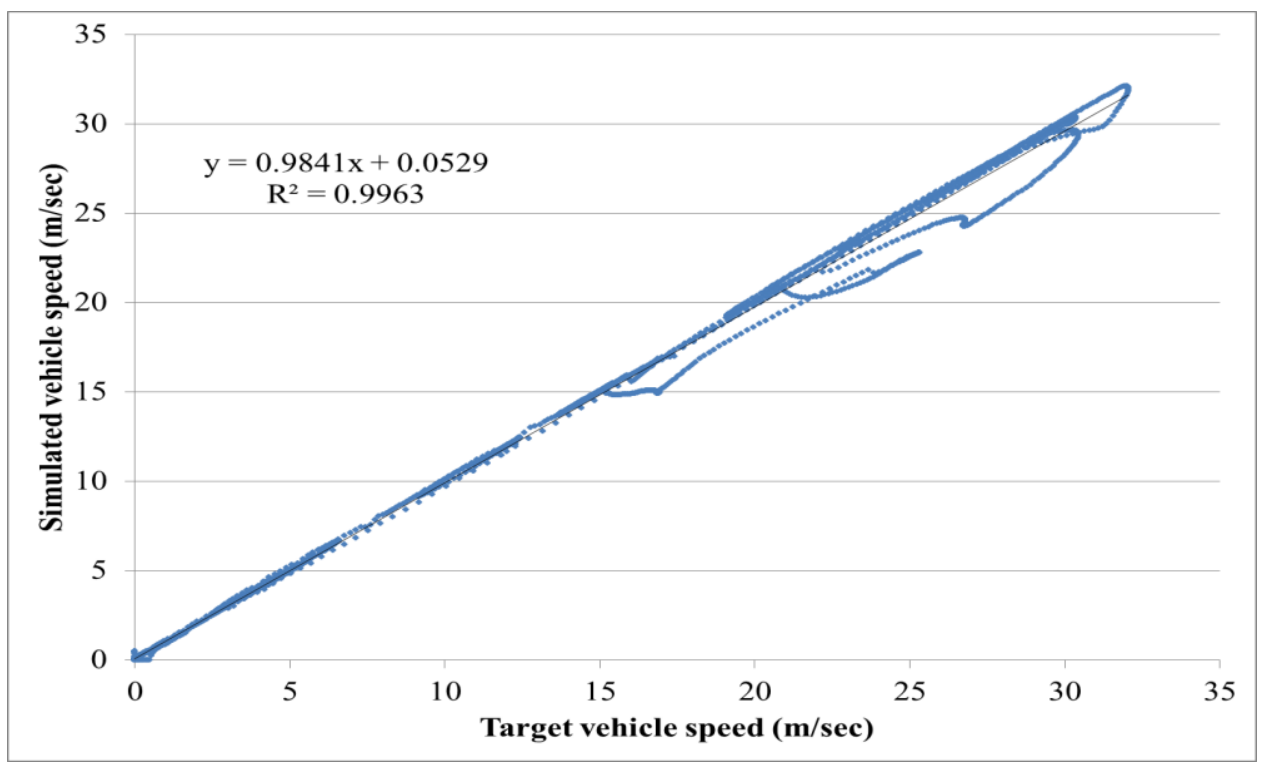

Figure A.12: Speed simulation results goodness for a HEV with ITS model at Sab2BM real world driving cycle 


\section{Appendix B: Evaluate the worth of parallel heavy duty HEV relative to conventional heavy duty vehicles}

Since the hybrid electric vehicle consider the captured energy as important alternative energy source, the terrain types and vehicle activity represent the most important factor to consider. Figures (B.1-B.5) show the fuel economy , fuel economy saving, engine efficiency, and regenerative braking energy for parallel HEV ( $150 \mathrm{~kW}$ electric motor, $10 \mathrm{kWh}$ battery energy, and 50\%load) comparing to conventional vehicle over different real world and chassis driving cycles.

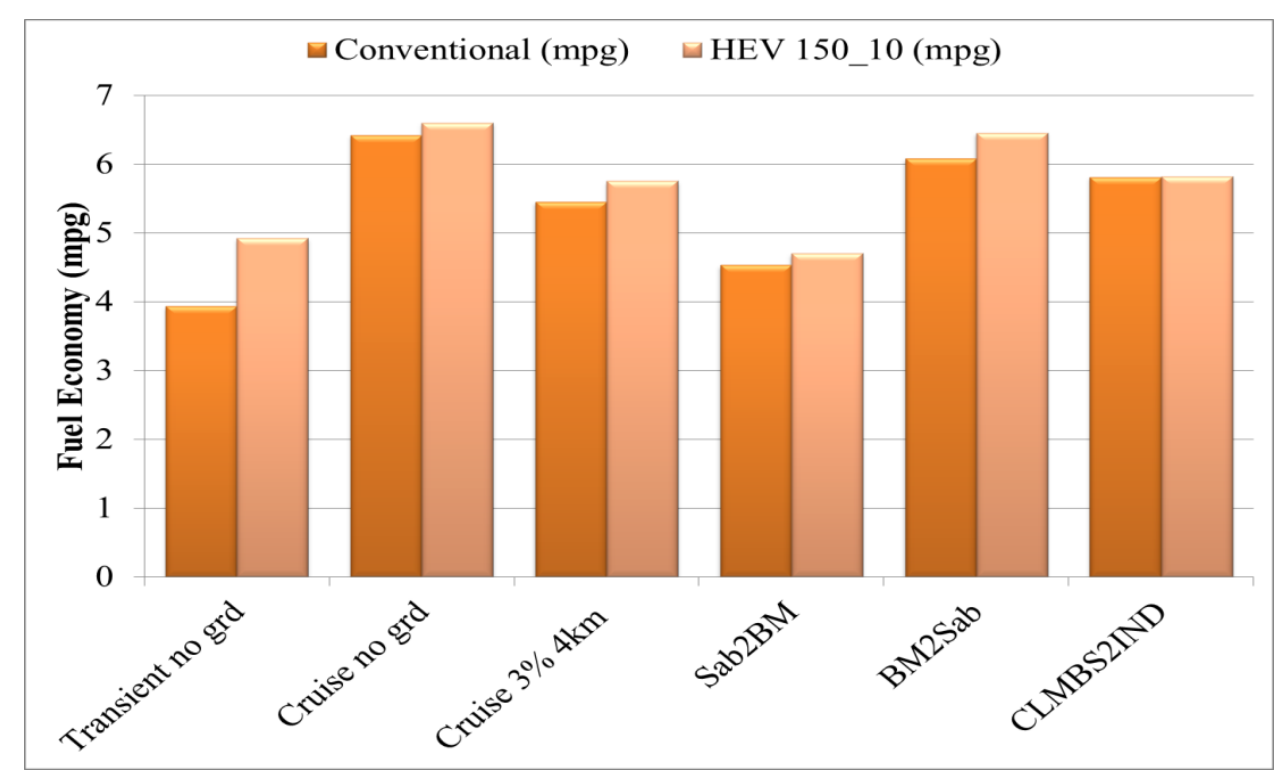

Figure B.1: Fuel economy for parallel HEV relative to conventional vehicle over different terrain types 


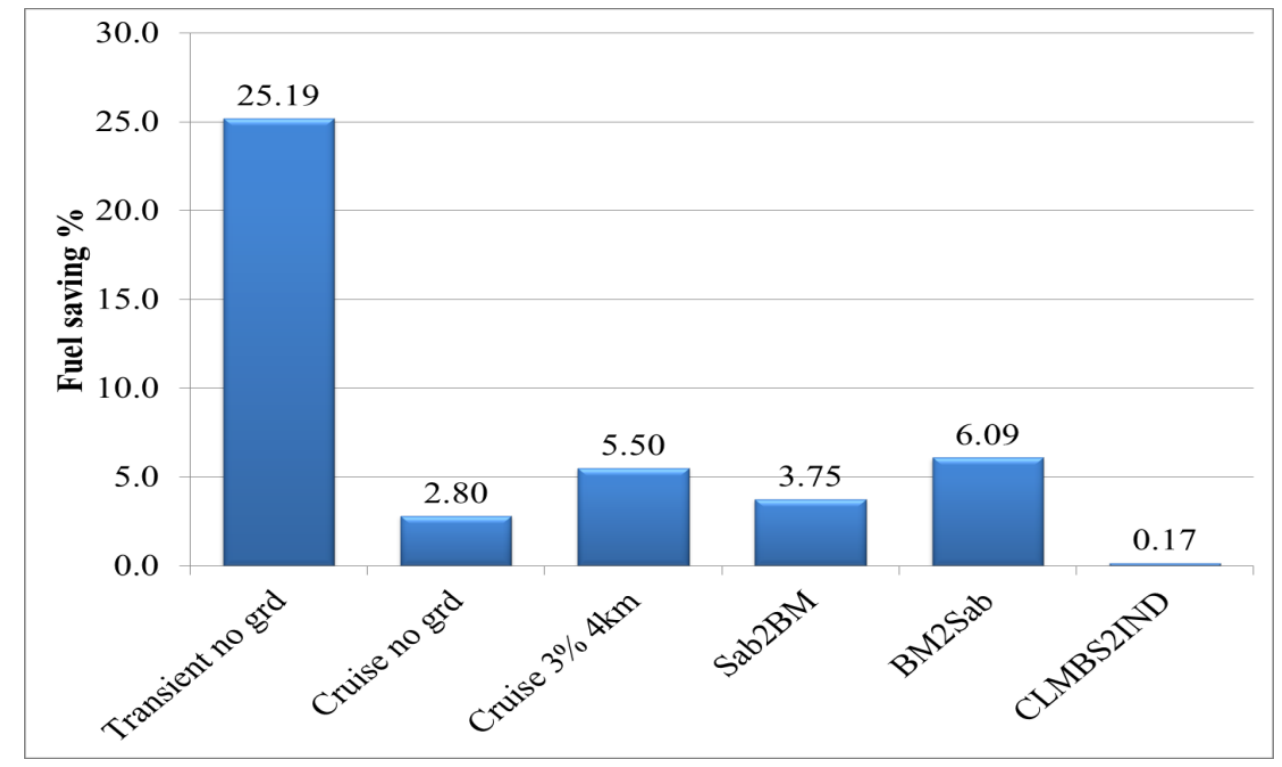

Figure B.2: Fuel economy saving for parallel HEV relative to conventional vehicle over different terrain types

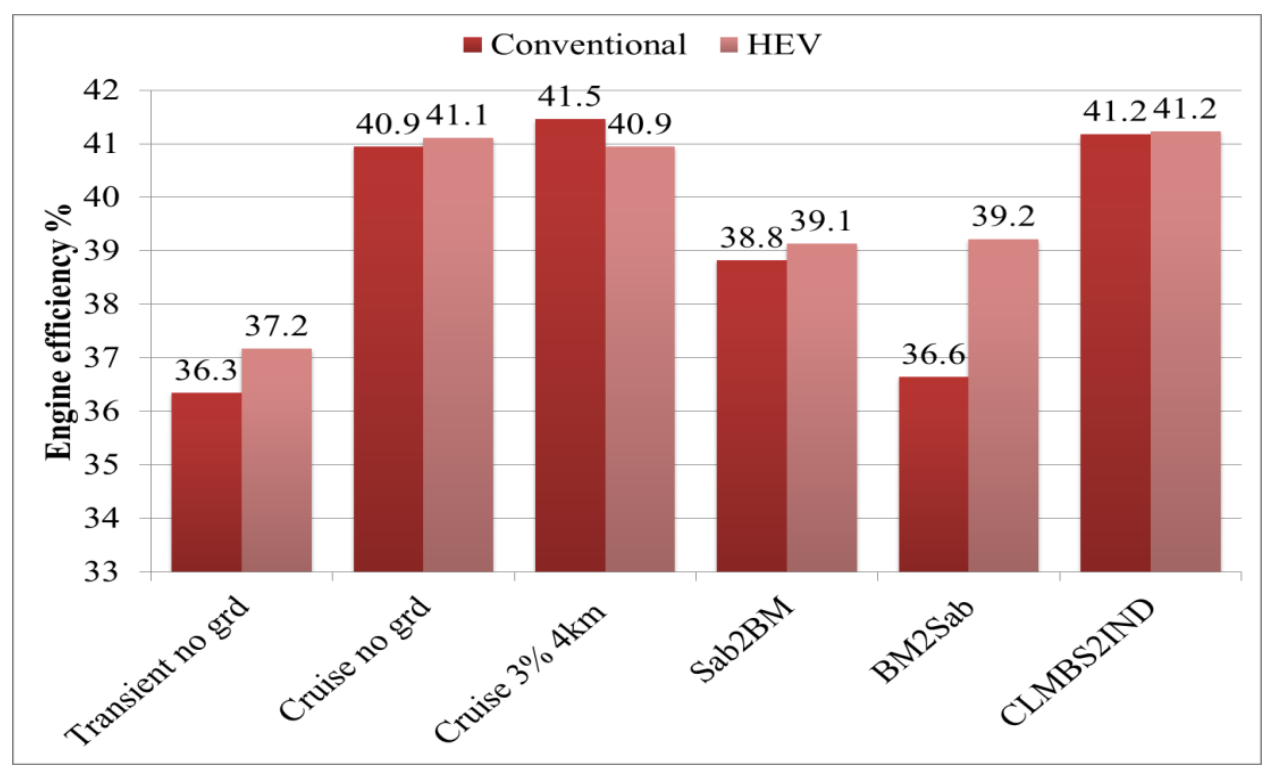

Figure B.3: Engine efficiency change for parallel HEV relative to conventional vehicle over different terrain types 


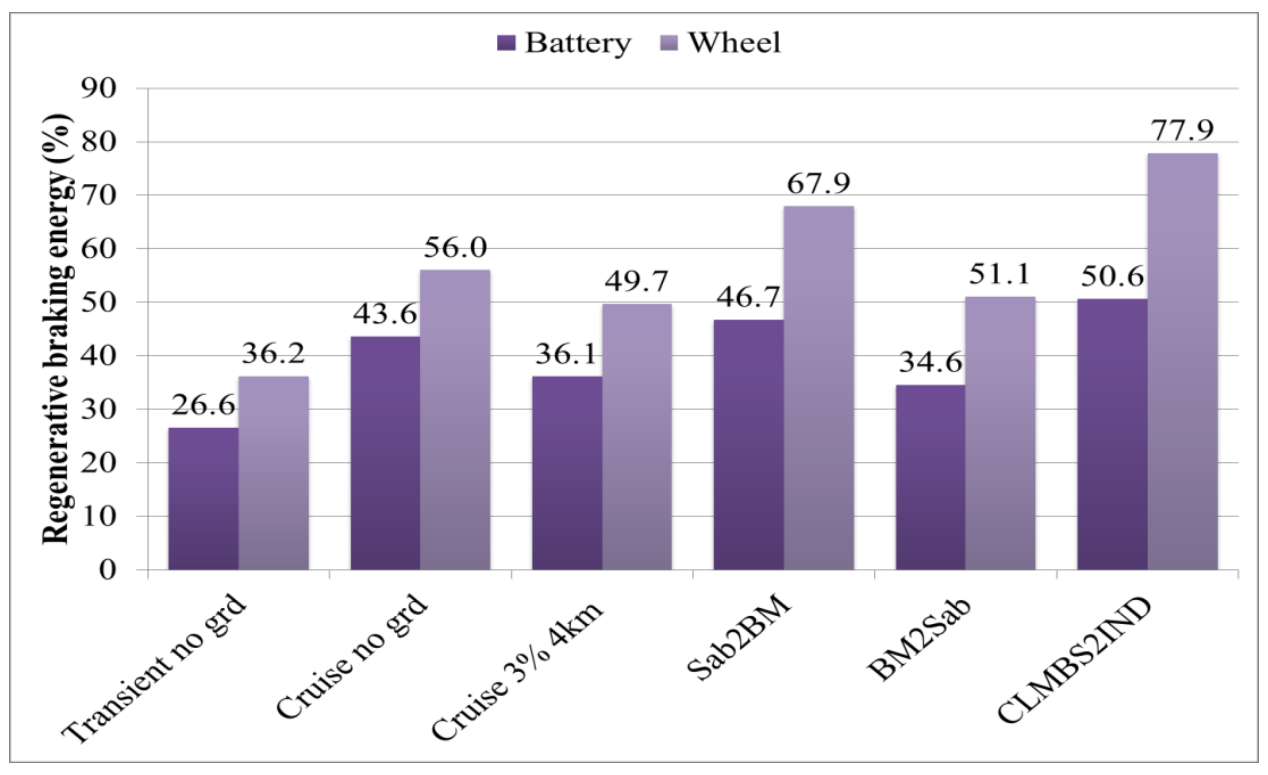

Figure B.4: Regenerative braking energy (\%) for parallel HEV relative to conventional vehicle over different terrain types

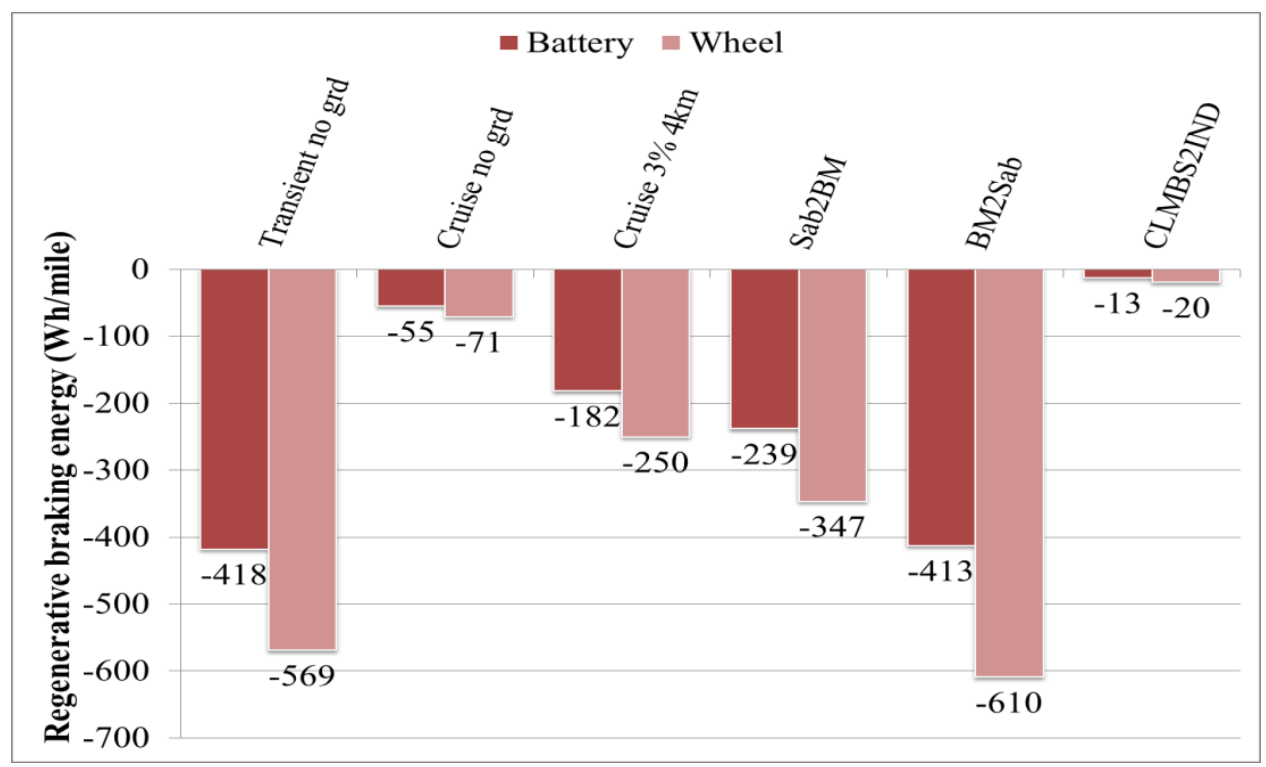

Figure B.5: Regenerative braking energy (Wh /mile) for parallel HEV relative to conventional vehicle over different terrain types 


\section{Appendix C: Energy losses and equipment efficiencies}

Figures (C.1 and C.2) show the impact of looking-ahead strategy on the forward and reverse energy losses. The most energy losses in the forward were from the engine, but the most energy losses in the reverse were from the electric generator. Figures (C.3 and C.4) show the forward and reverse total efficiencies and energy balance for all equipment in the parallel HEV model. These results were for parallel HEV with 150kW_20kWh equipment sizes and full load (36,000 $\mathrm{kg}$ ) at highway and long ascending hill (3\%) driving cycle. Total forward energy losses decreased due to looking-ahead strategy, in contrast the total reverse energy losses increased. However, the net change of energy losses was impacted positively. This run was for highway and uphill driving cycles. These figures collected from Autonomie simulation software which is using data for the ESS provided by "A.Burke (UC Davis)"
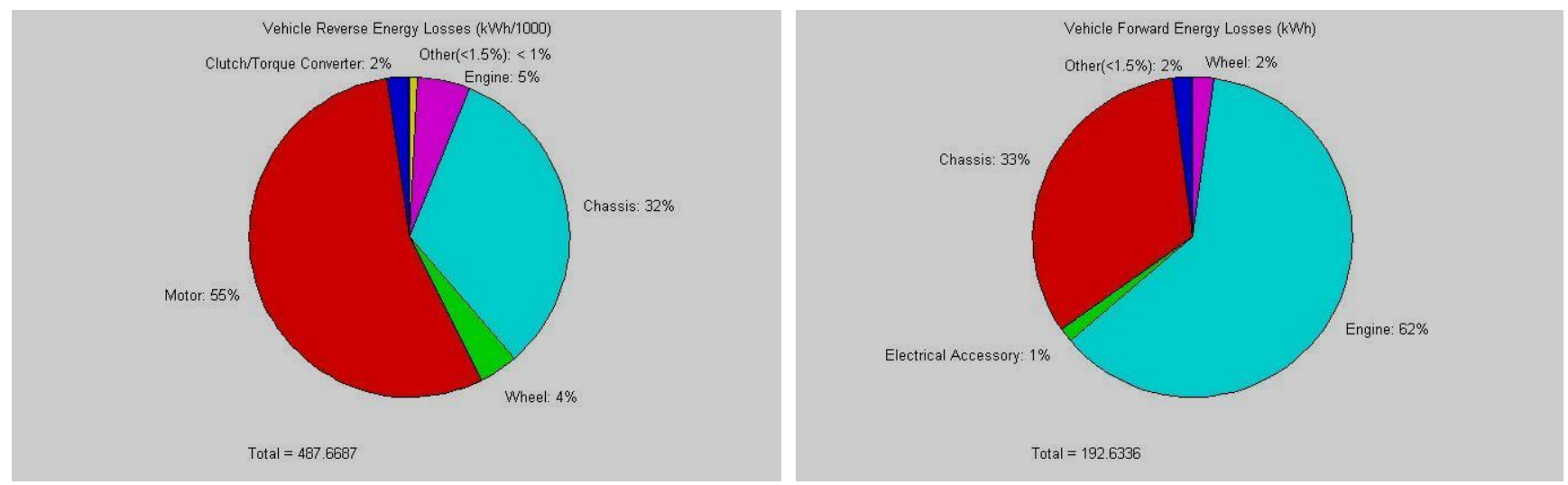

Figure C.1: Forward and reverse energy losses from the parallel HEV model equipment without looking-ahead strategy.
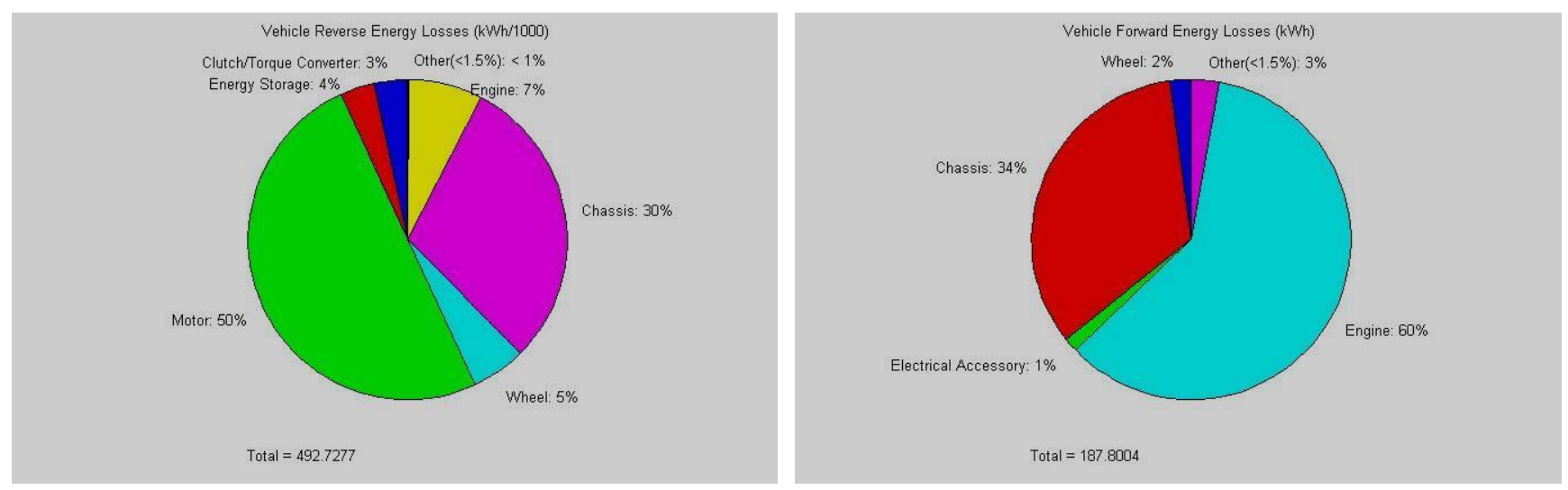

Figure C.2: Forward and reverse energy losses from the parallel HEV model equipment without looking-ahead strategy. 


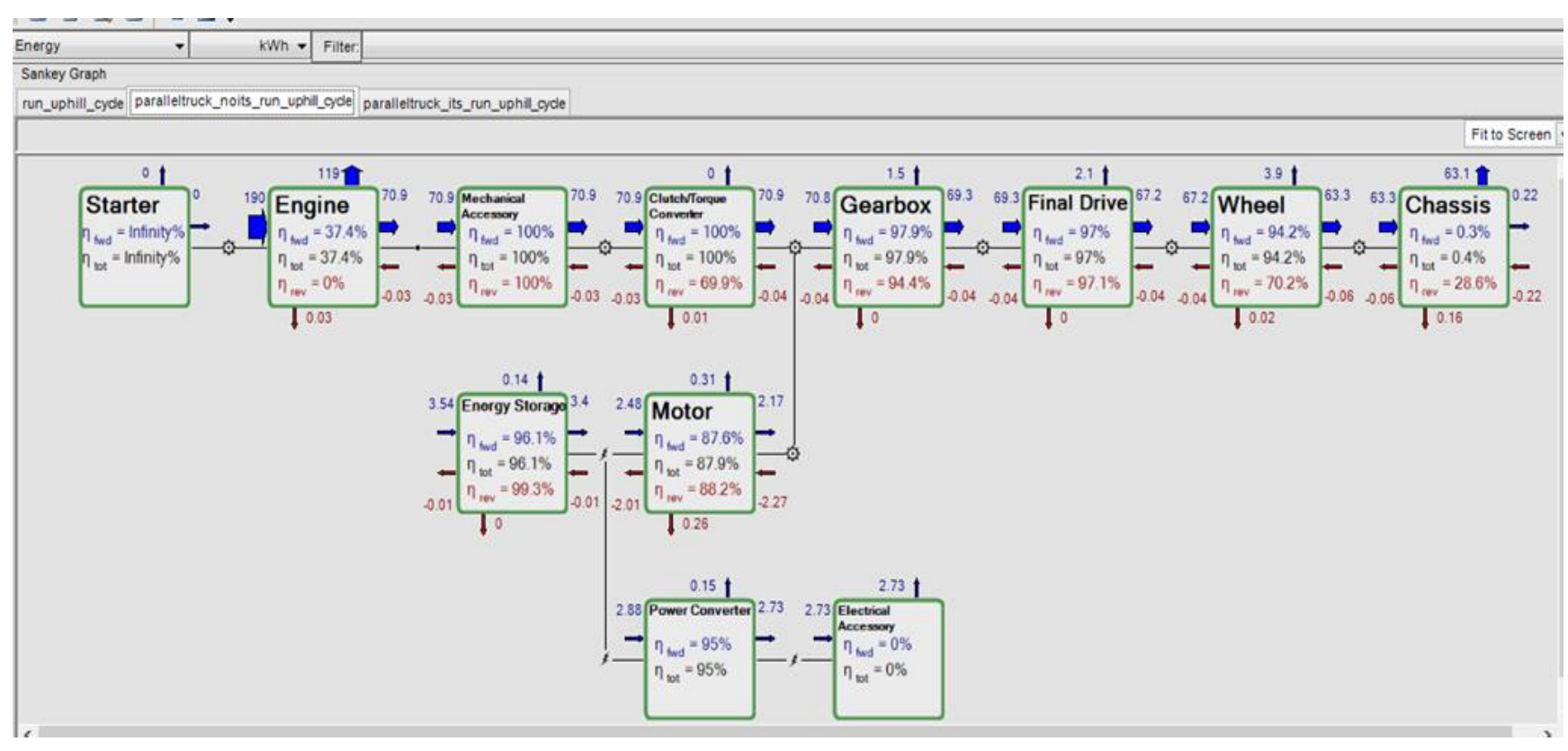

Figure C.3: Equipment efficiencies and energy balance for parallel HEV model without looking-ahead strategy

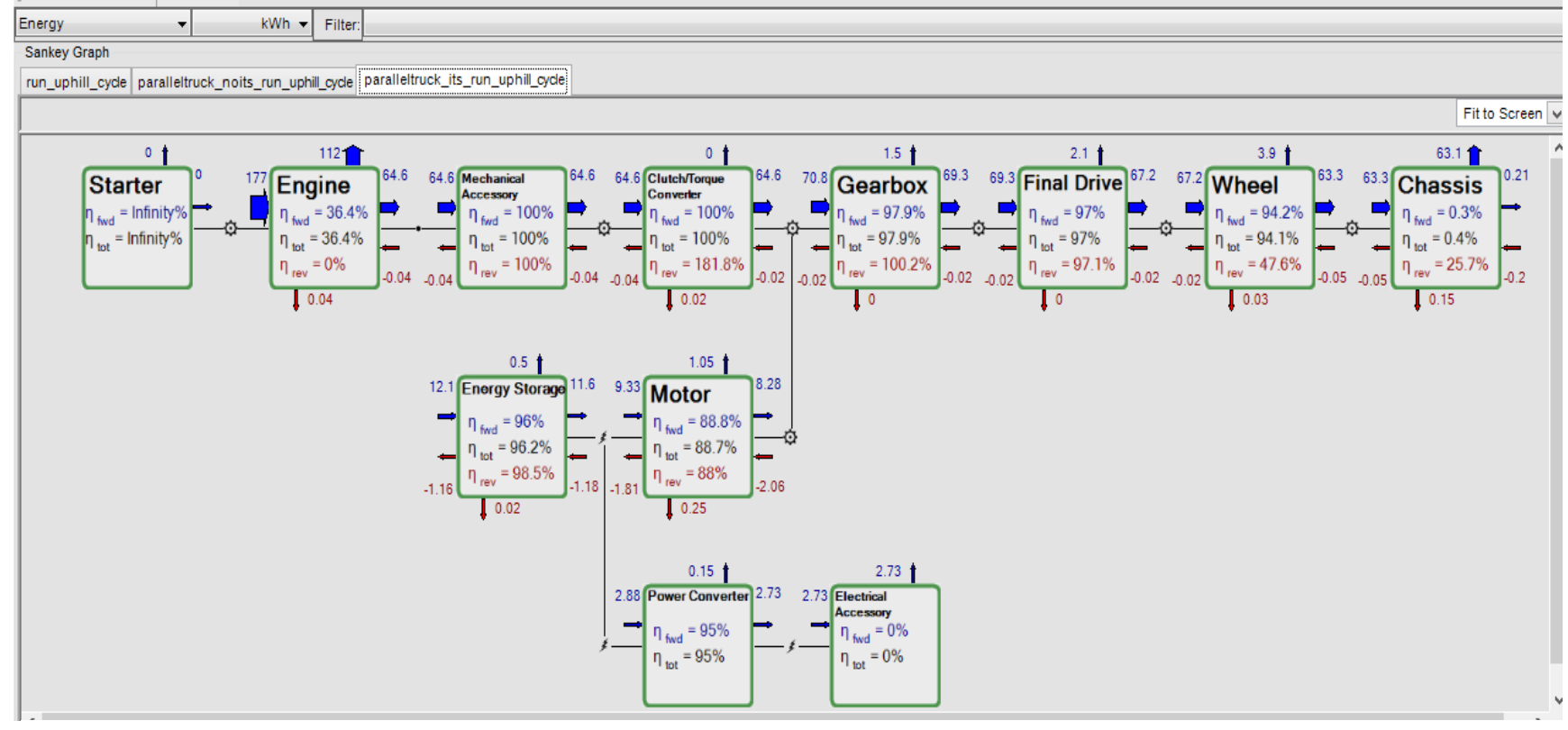

Figure C.4: Equipment efficiencies and energy balance for parallel HEV model with looking-ahead strategy 


\section{Following is the initial file for battery NiMH}

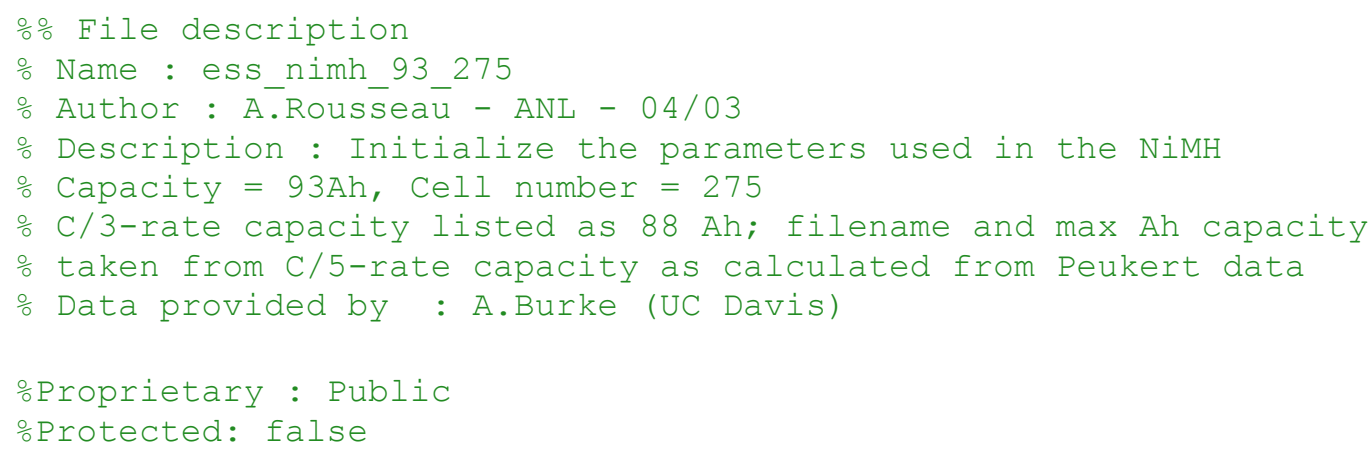


ess.plant.init.eff_coulomb.idx1_temp = ess.plant.init.temp_index;

ess.plant.init.eff_coulomb.map $=\left[\begin{array}{lll}1 & 1 & 1\end{array}\right]$; $\frac{\circ}{0}$ average coulombic (a.k.a. amp-hour) efficiency below, indexed by ess.plant.init.temp_index

o cell's resistance to being discharged, indexed by ess.plant.init.soc_index and ess.plant.init.temp index

ess.plant.init.rint_dis.idxl_temp = ess.plant.init.temp_index;

ess.plant.init.rint_dis.idx2_soc = ess.plant.init.soc iñdex;

ess.plant.init.rint_dis.map $=\overline{[}$

$1.601 .221 .07 \quad 1.06 \quad 1.051 .04 \quad 1.04 \quad 1.041 .041 .041 .04$

$1.601 .221 .07 \quad 1.06 \quad 1.05 \quad 1.04 \quad 1.04 \quad 1.04 \quad 1.04 \quad 1.041 .04$

$1.601 .221 .071 .061 .051 .04 \quad 1.04 \quad 1.041 .041 .041 .04 \quad$ ]/1000; $\%$ (ohm)

\% cell's resistance to being charged, indexed by ess.plant.init.soc_index and ess.plant.init.temp_index

ess.plant.init.rint_chg.idx1_temp = ess.plant.init.temp_index;

ess.plant.init.rint chg.idx2 ${ }^{-}$soc $=$ess.plant.init.soc index;

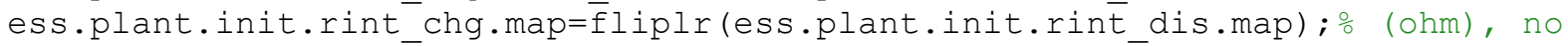
other data available

o cell's open-circuit (a.k.a. no-load) voltage, indexed by ess.plant.init.soc_index and ess.plant.init.temp_index

ess.plant.init.voc.idxl_temp = ess.plant.init.temp_index;

ess.plant.init.voc.idx2_soc = ess.plant.init.soc_iñdex;

ess.plant.init.voc.map $=\overline{[}$

$\begin{array}{llllllllllllll}1.12 & 1.222 & 1.241 & 1.265 & 1.285 & 1.296 & 1.317 & 1.338 & 1.358 & 1.379 & 1.4\end{array}$

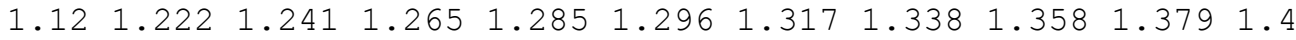

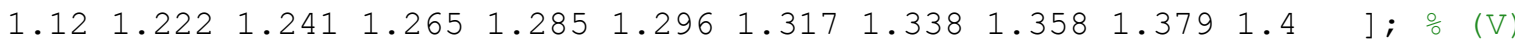

\% Max current and power when charging/discharging

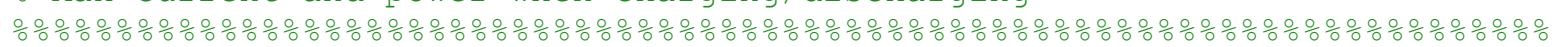

ess.plant.init.curr_chg_max = -max(max((ess.plant.init.volt_max-

ess.plant.init.voc.map)./ess.plant.init.rint_chg.map));

ess.plant.init.curr_dis_max = max (max( (ess.plant.init.voc.map-

ess.plant.init.volt_min) ./ess.plant.init.rint_dis.map));

ocheck the ess.plant.init.pwr_chg \& ess.plant.init.pwr_dis because they're a vector and in the database for the plot we

oneed maps

ess.plant.init.pwr_chg.idx1_soc = ess.plant.init.soc_index;

ess.plant.init.pwr_dis.idx1_soc = ess.plant.init.soc_index;

ess.plant.init.pwr_chg.map = -max((ess.plant.init.volt_max-

ess.plant.init.voc.map).*ess.plant.init.volt_max./ess.plant.init.rint_chg.map

); $\circ$ per cell

ess.plant.init.pwrdis.map = max( (ess.plant.init.voc.map-

ess.plant.init.volt_min). *ess.plant.init.volt_min./ess.plant.init.rint_dis.ma

p); ㅇper cell

\% gain factor to modify ess.plant.init.pwr_chg and ess.plant.init.pwr_dis

discharge is brought to 0 at low soc and charge is brought to 0 at high soc

ess.plant.init.pwr_chg.map

ess.plant.init.pwr_chg.map. *double(ess.plant.init.soc index $<=$

ess.plant.init.soc_max);

ess.plant.init.pwr dis.map = ess.plant.init.pwr_dis.map.*

double (ess.plant.iñt.soc_index >= ess.plant.init.soc_min); 
ess.plant.init.pwr_chg_at_min_soc =

interp1 (ess.plant. $\overline{i n i t . s o \bar{c}}$ index, ess.plant.init.pwr_chg.map, ess.plant.init.so c_min); $\frac{\circ}{0}(0->1)$ Power at which battery is charged when soc is at its minimum ess.plant.init.pwr_dis_at_max_soc=

interpl (ess.plant. $\overline{i n i t . s o c}$ index, ess.plant.init.pwr_dis.map, ess.plant.init.so c_max $) ; \quad(0->1)$ Power at which battery is discharge d when soc is at its māximum

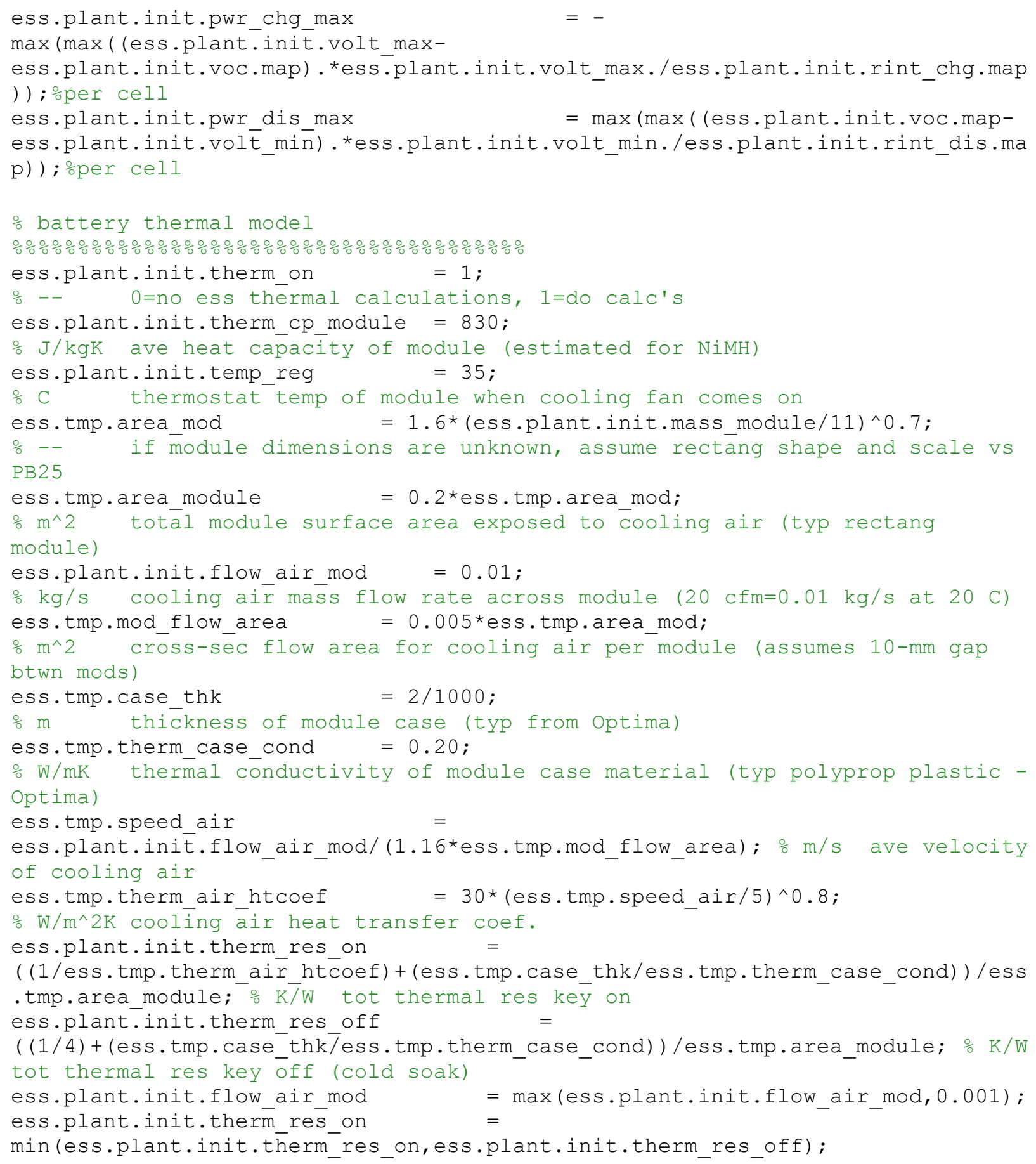




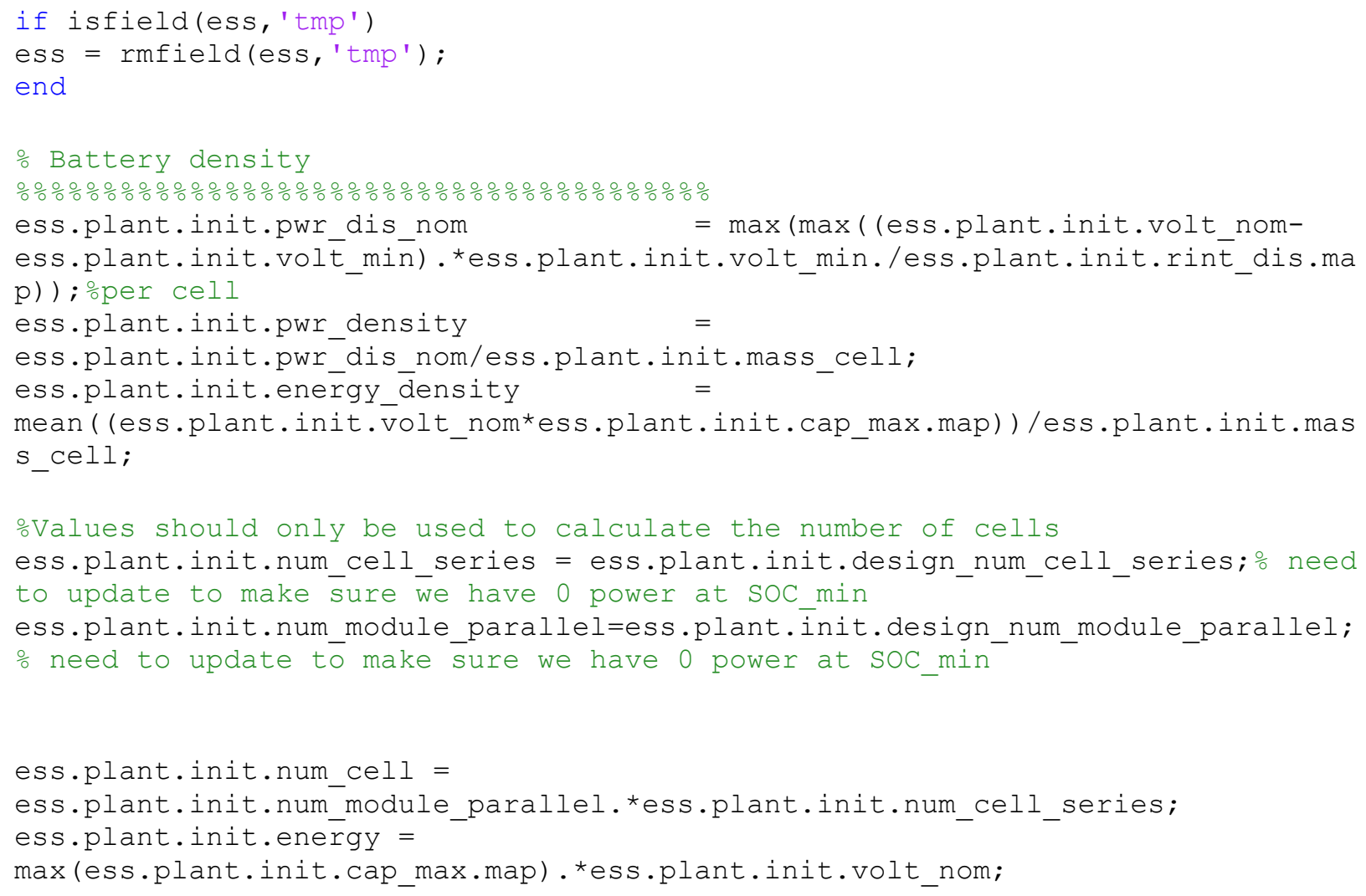




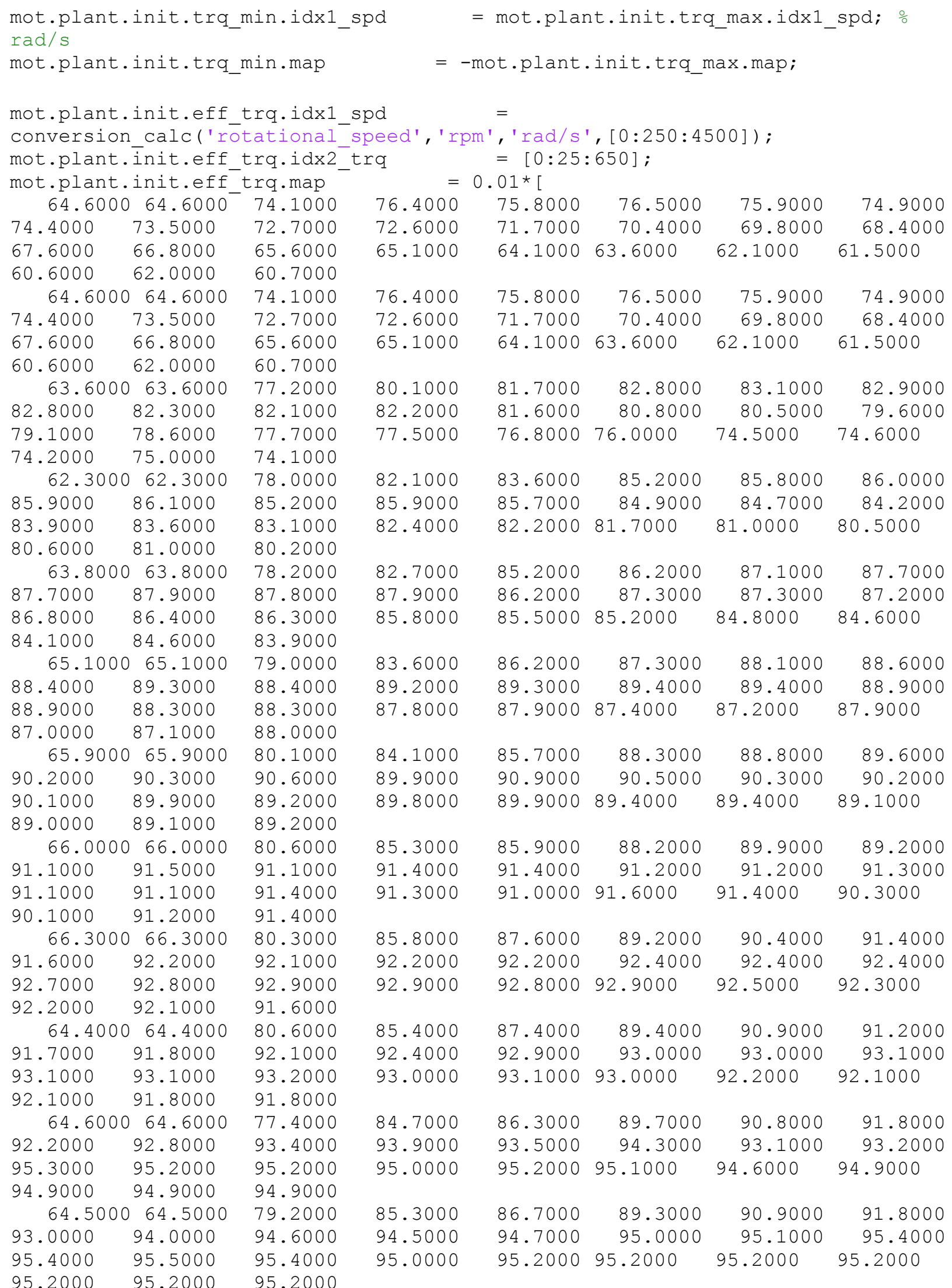




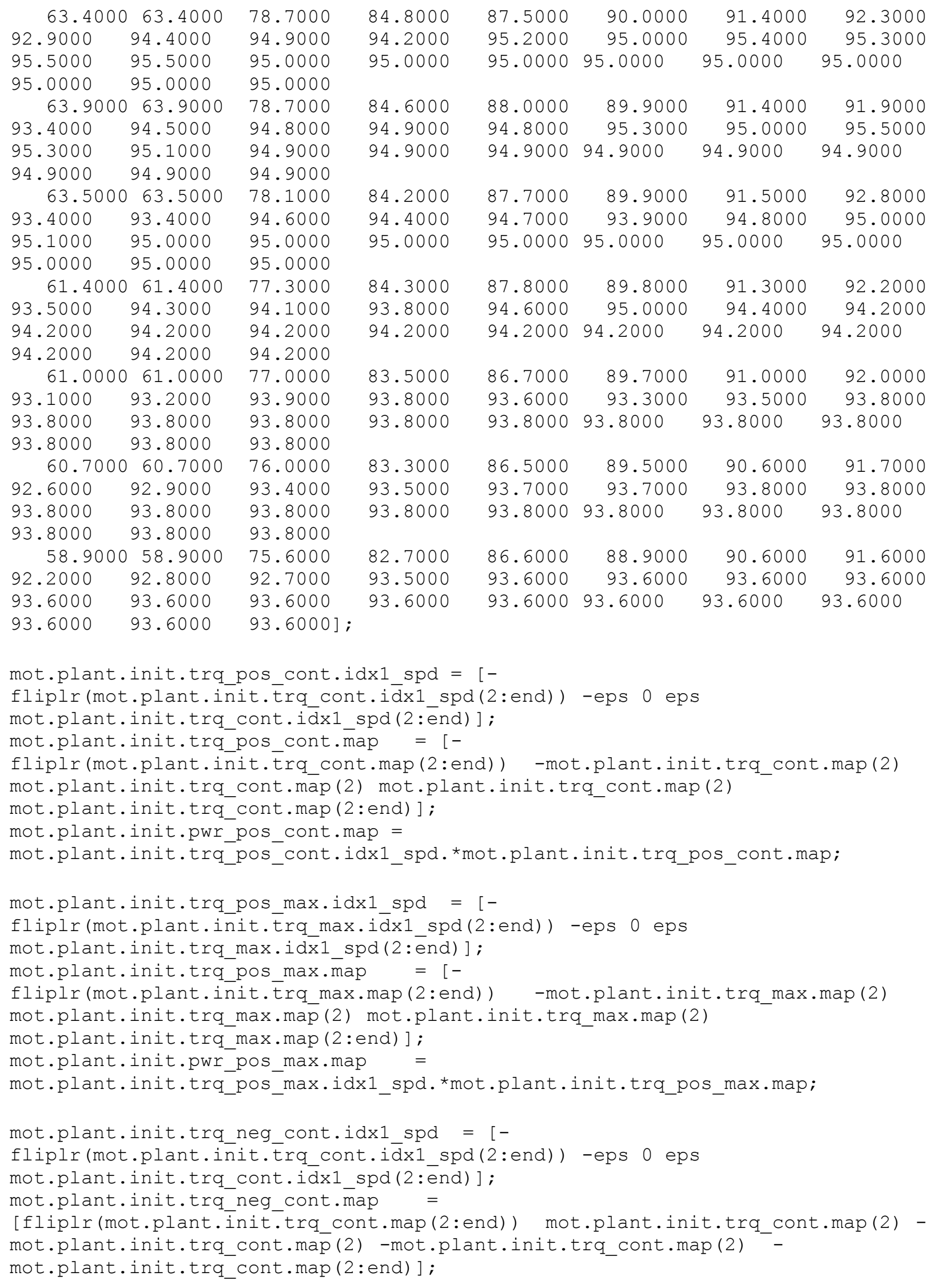




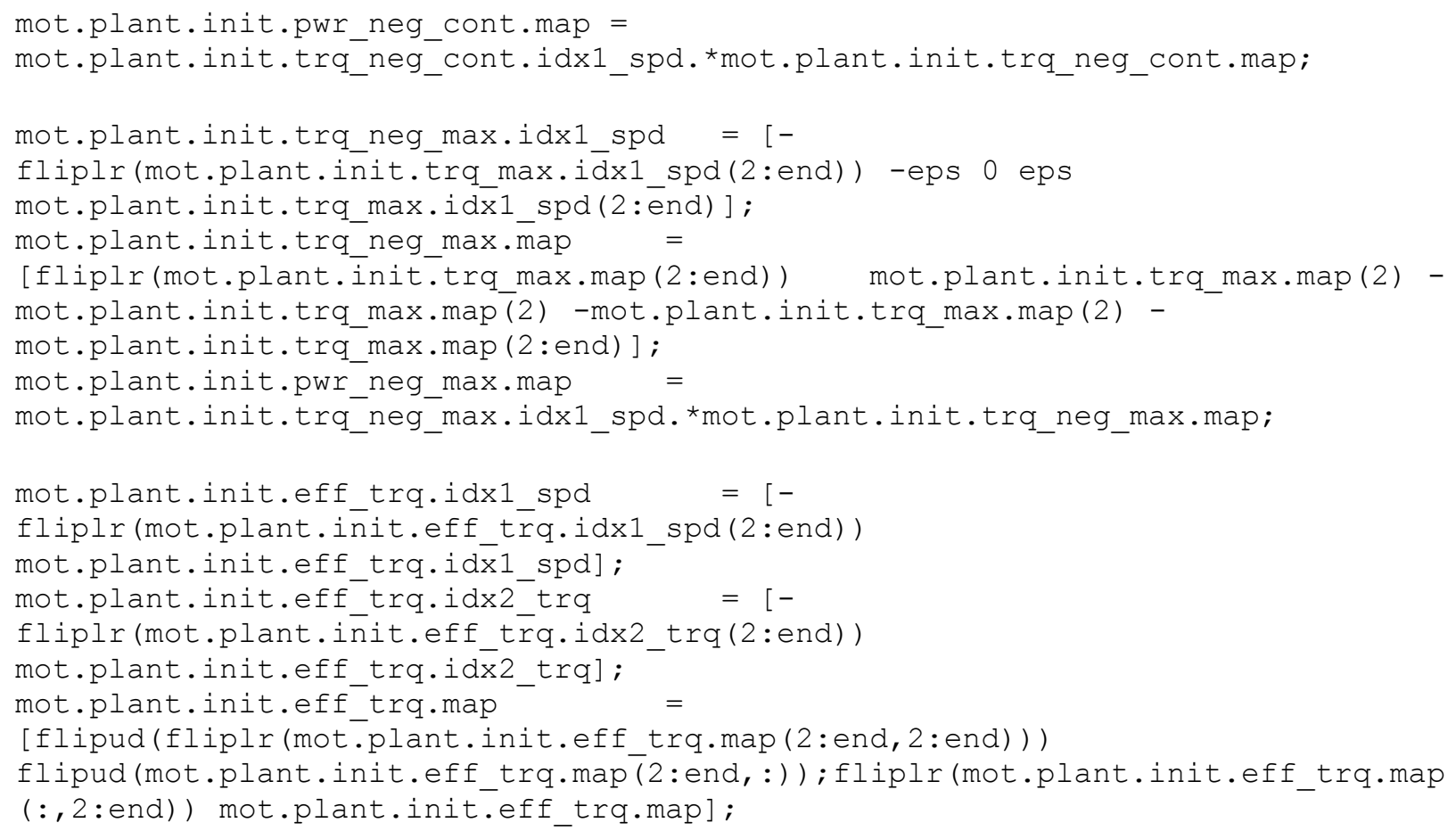




\section{Appendix D: Matlab program code built by the researcher}

\section{Code 1}

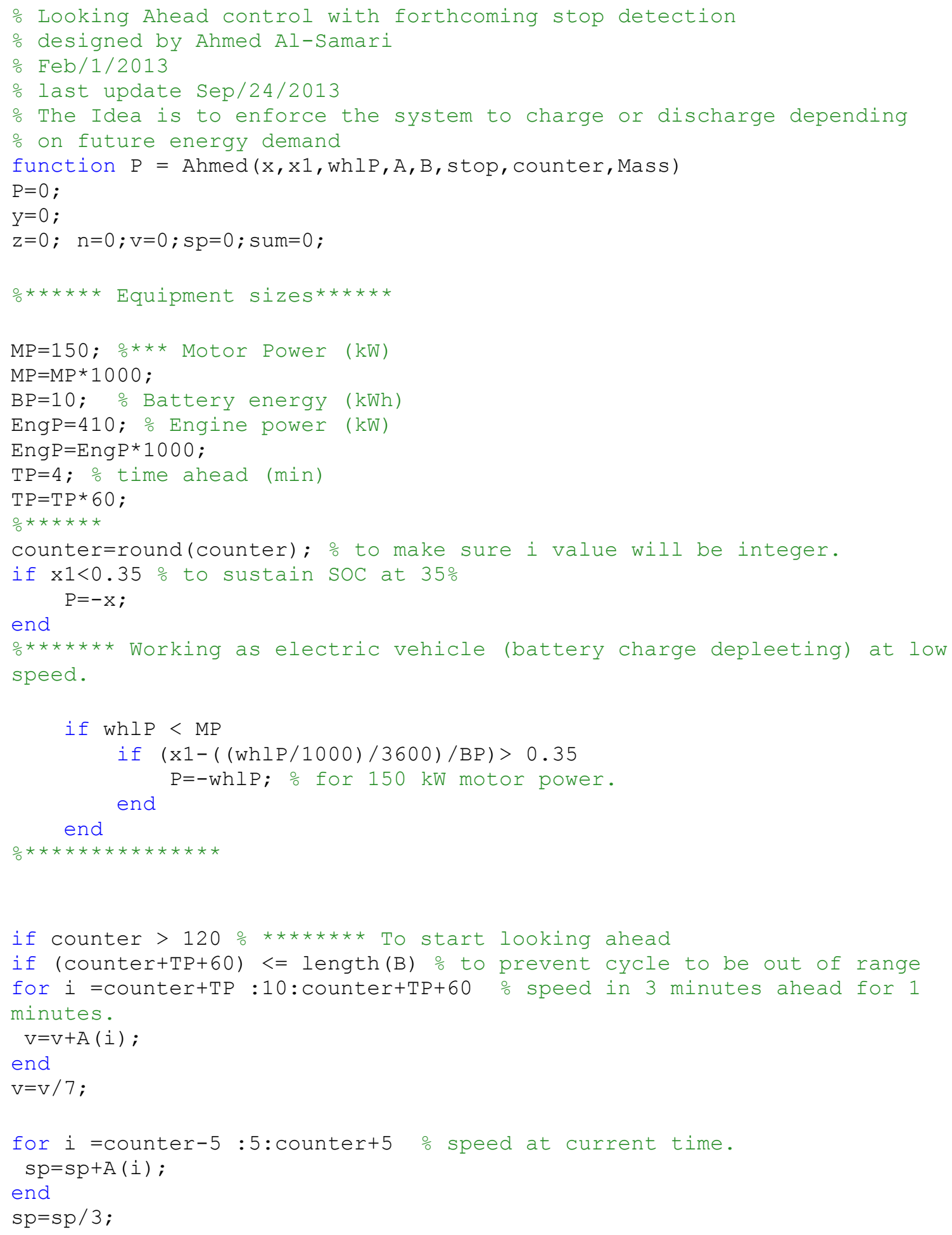




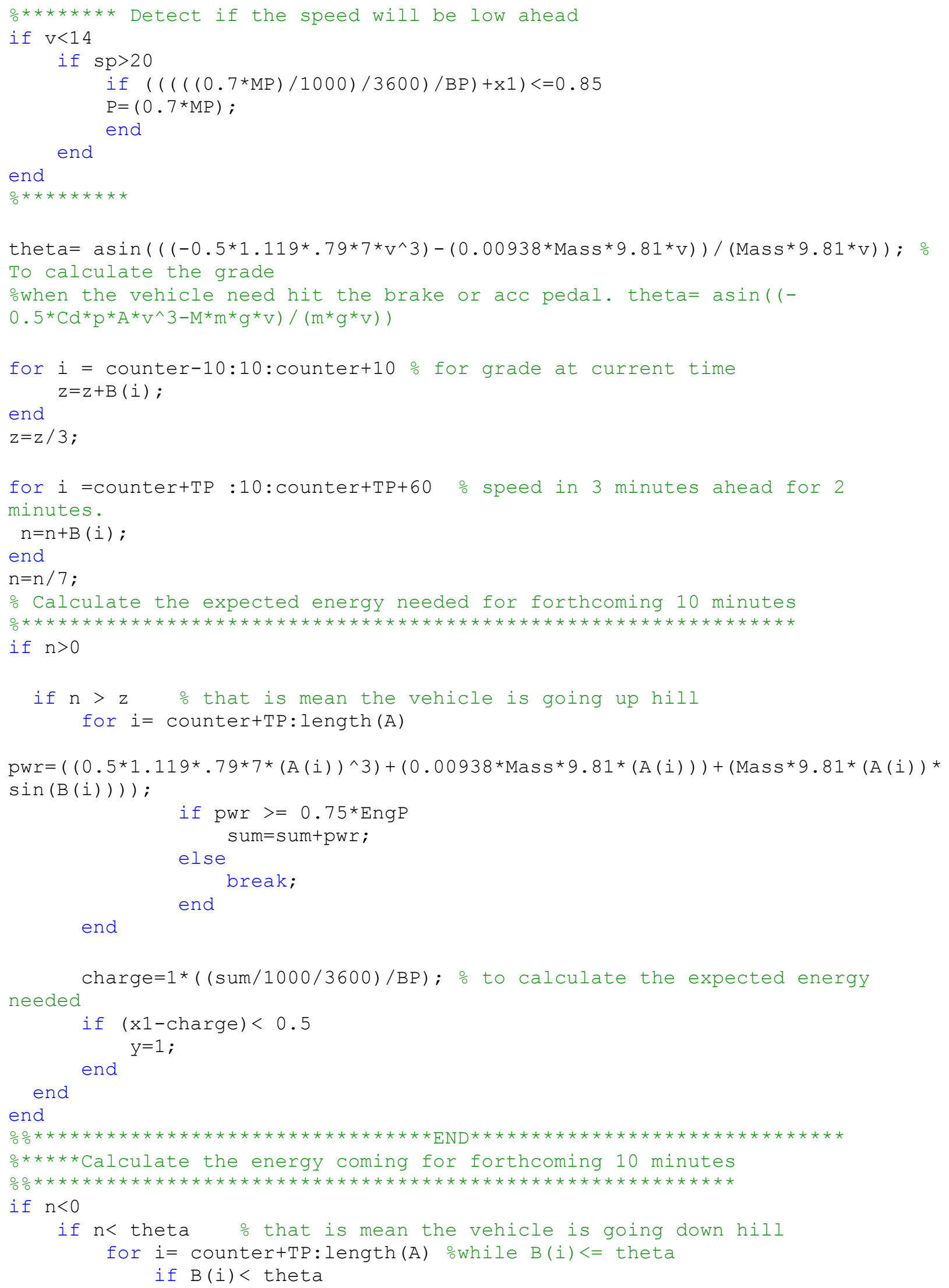




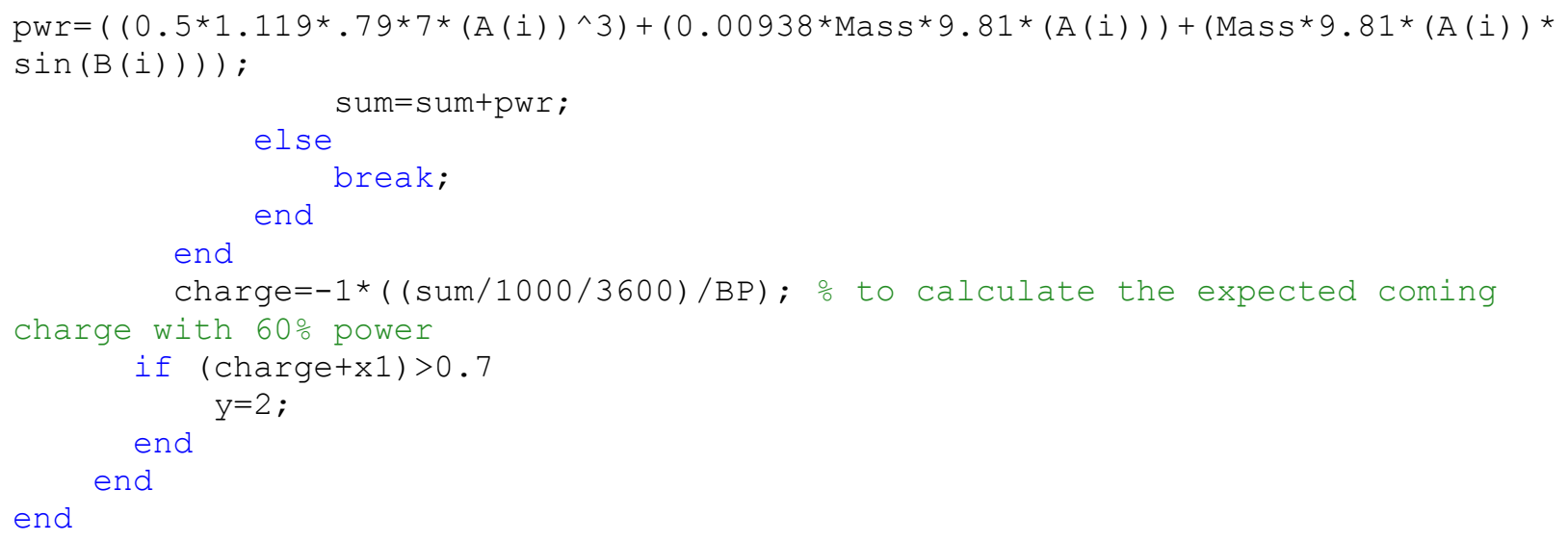




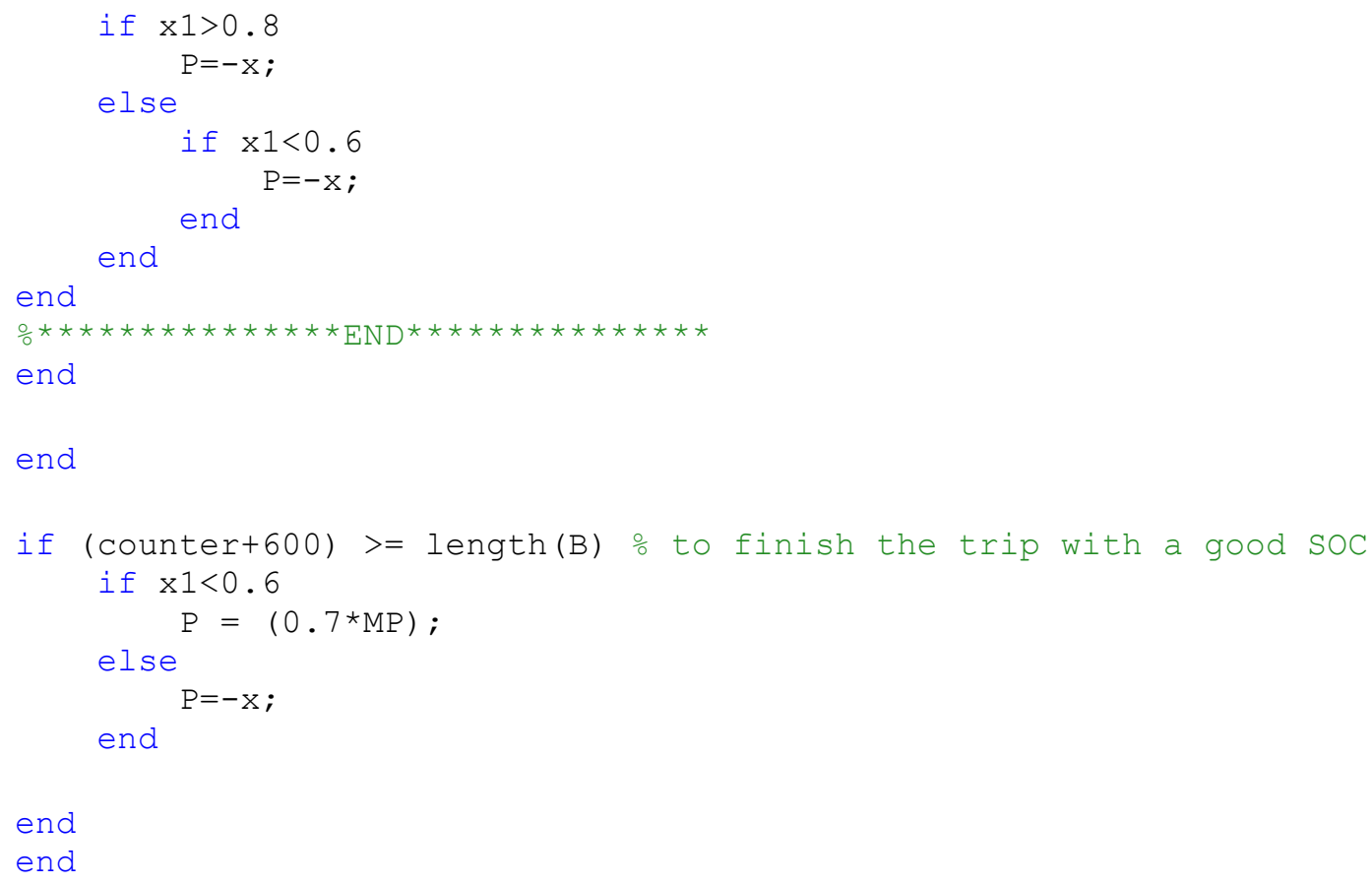

\section{Code 2}

oProgram to generate different road grade at different oscillations oThis program built by Ahmed Al-Samari 


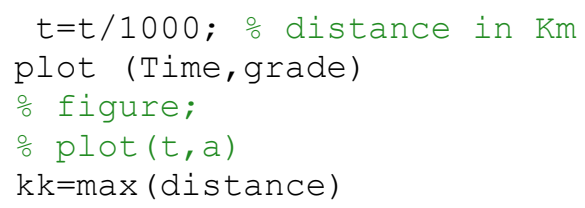

\section{Code 3}

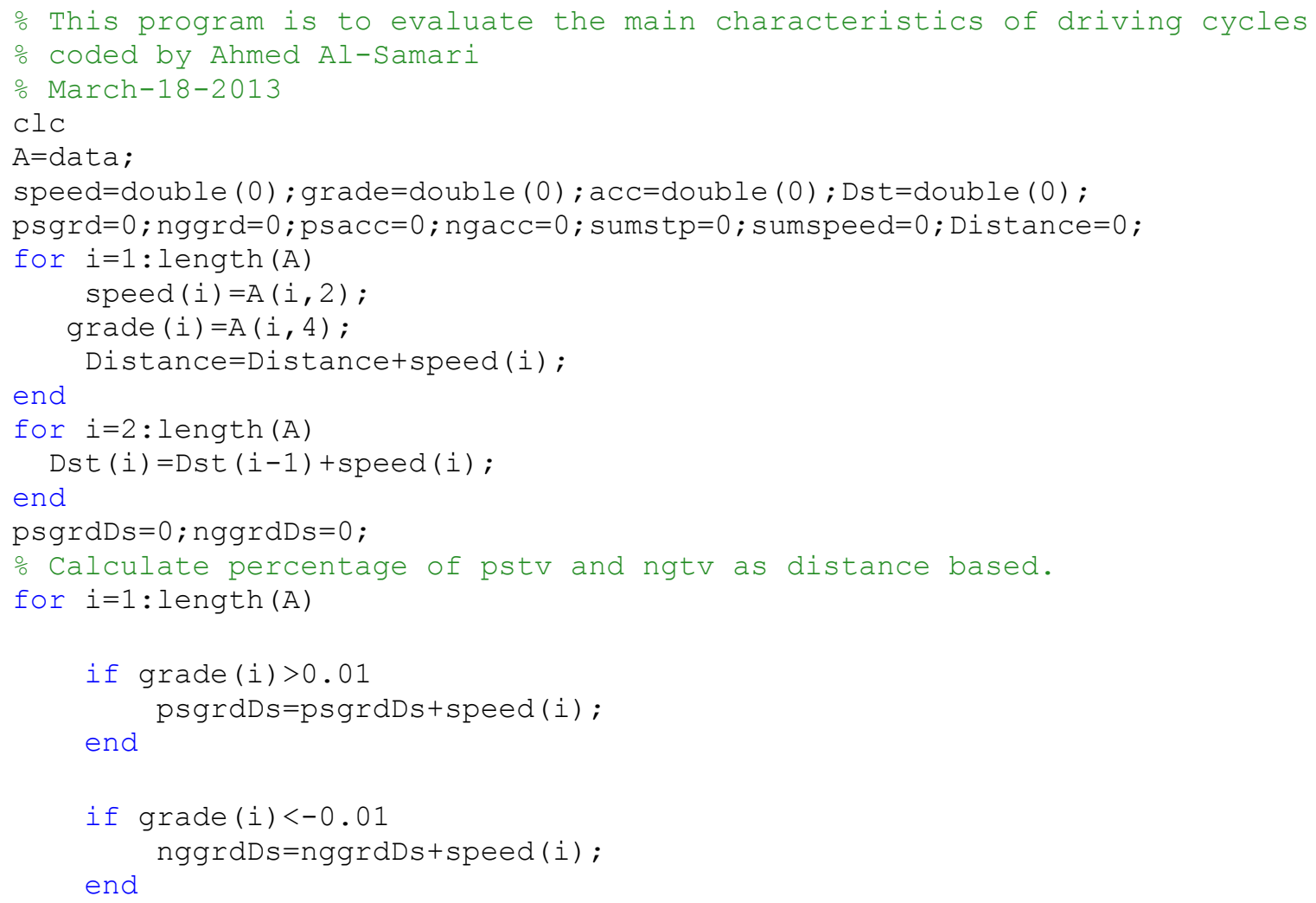




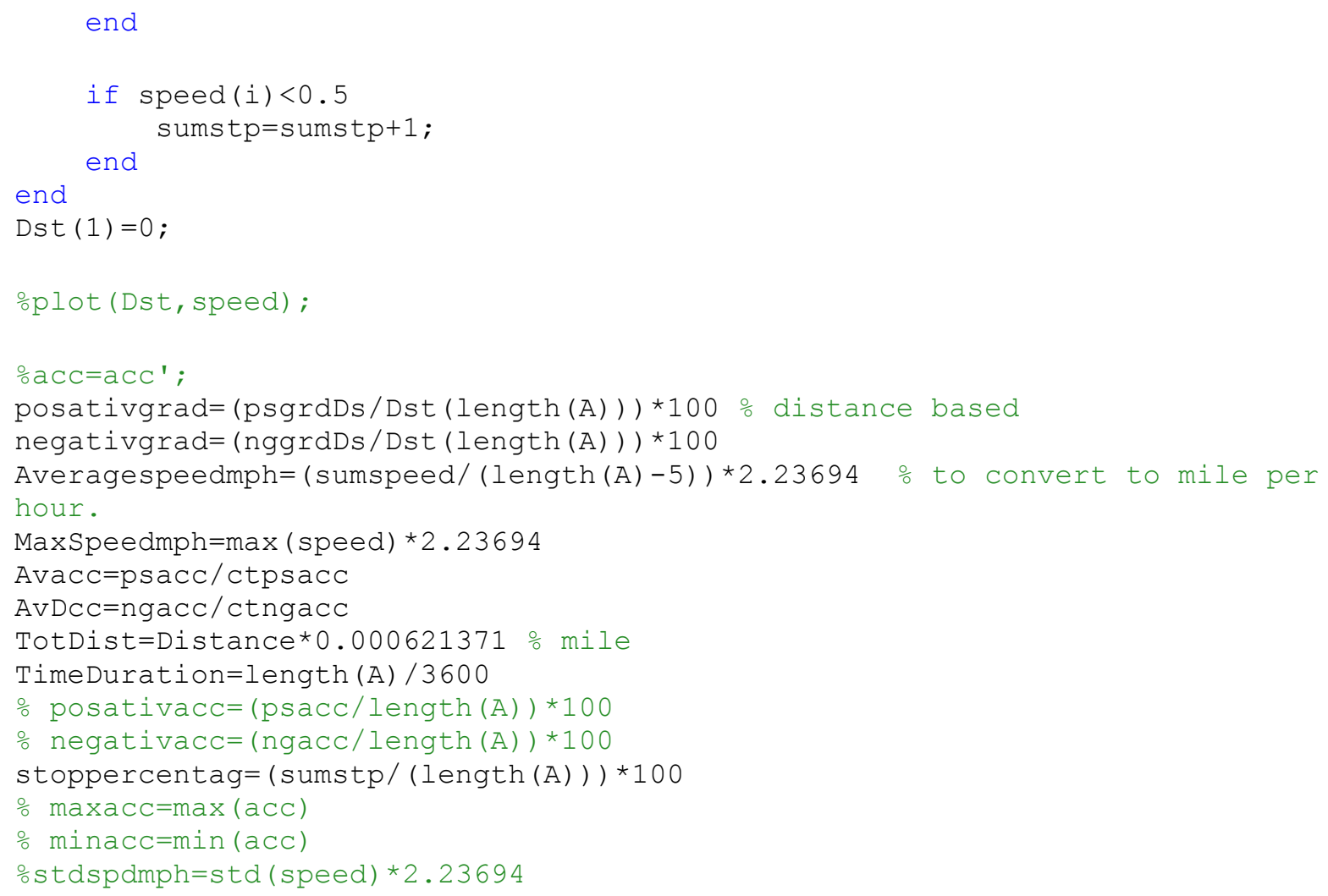

